KATHOLIEKE UNIVERSITEIT LEUVEN

FACULTEIT INGENIEURSWETENSCHAPPEN

DEPARTEMENT COMPUTERWETENSCHAPPEN

AFDELING INFORMATICA

Celestijnenlaan 200 A - B-3001 Leuven

\title{
Optimizing Compilation and Computational Complexity of Constraint Handling Rules
}

Promotor :

Prof. Dr. B. DEMOEN
Proefschrift voorgedragen tot het behalen van het doctoraat in de ingenieurswetenschappen

door

Jon SNEYERS 

KATHOLIEKE UNIVERSITEIT LEUVEN

FACULTEIT INGENIEURSWETENSCHAPPEN

DEPARTEMENT COMPUTERWETENSCHAPPEN

AFDELING INFORMATICA

Celestijnenlaan 200 A - B-3001 Leuven

\section{Optimizing Compilation and Computational Complexity of Constraint Handling Rules}

Jury :

Prof. Dr. ir. P. Van Houtte, voorzitter

Prof. Dr. B. Demoen, promotor

Prof. Dr. ir. M. Bruynooghe

Prof. Dr. ir. F. Piessens

Prof. Dr. T. Frühwirth (Universität UIm)

Prof. Dr. F. Fages (INRIA Paris-Rocquencourt)

U.D.C. $681.3 * \mathrm{D} 3$
Proefschrift voorgedragen tot het behalen van het doctoraat in de ingenieurswetenschappen

door

Jon SNEYERS 
(C)Katholieke Universiteit Leuven - Faculteit Ingenieurswetenschappen Arenbergkasteel, B-3001 Heverlee (Belgium)

Alle rechten voorbehouden. Niets uit deze uitgave mag worden vermenigvuldigd en/of openbaar gemaakt worden door middel van druk, fotocopie, microfilm, elektronisch of op welke andere wijze ook zonder voorafgaande schriftelijke toestemming van de uitgever.

All rights reserved. No part of the publication may be reproduced in any form by print, photoprint, microfilm or any other means without written permission from the publisher.

$\mathrm{D} / 2008 / 7515 / 107$

ISBN 978-90-5682-997-1 


\title{
Optimizing Compilation and Computational Complexity of Constraint Handling Rules
}

\author{
Jon Sneyers
}

November 2008

\begin{abstract}
Constraint Handling Rules (CHR) is a very-high-level declarative programming language based on concurrent multiset rewrite rules that are conditional, multiheaded, and committed-choice. Originally designed in the early 1990s as a specialpurpose programming language for adding user-defined constraint solvers to a host language, CHR has evolved over the last decade into a powerful and elegant general-purpose language with a wide spectrum of application domains.

Computational complexity theory is the study of scalability of computer programs in terms of the computational resources they require - in particular, time (cpu usage) and space (memory usage).

In this dissertation we investigate the CHR programming language from the point of view of computational complexity theory. The first part introduces complexity theory, CHR, and CHR compilation. In the second part, we improve the state of the art by proposing and implementing several compiler optimizations. We confirm experimentally that these optimizations improve both the time and space complexity of CHR programs.

Finally, in the third part of this dissertation, we prove a "complexity-wise completeness" result. We demonstrate that the CHR language and state-of-theart CHR systems (that implement the compiler optimizations of the previous part) are sufficiently efficient in the following precise sense: every algorithm can be implemented in CHR and be executed with the optimal asymptotic time and space complexity.
\end{abstract}




\section{Contents}

Abstract $\quad$ i

Table of Contents $\quad$ ii

List of Tables . . . . . . . . . . . . . . . . . . ix

List of Figures . . . . . . . . . . . . . . . . . $\mathrm{x}$

List of Listings . . . . . . . . . . . . . . . . . . . xii

$\begin{array}{ll}\text { Preface } & \text { xv }\end{array}$

1 Introduction $\quad 1$

1.1 Context .......................... 1

1.1.1 Declarative programming ................. 2

1.1.2 Constraint logic programming . . . . . . . . . . . . . 3

1.1.3 Constraint Handling Rules . . . . . . . . . . . . . . 5

1.2 Goals and contributions . . . . . . . . . . . . . . 6

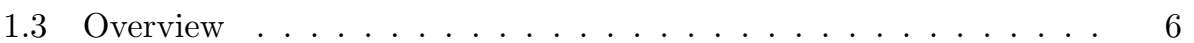

1.3.1 Part I - Background . . . . . . . . . . . . . 6

1.3.2 Part II - Optimizing Compilation . . . . . . . . . . . 7

1.3.3 Part III - Computability and Complexity . . . . . . . . . 7

1.3.4 Chapter dependencies ............... . . 7

1.4 Bibliographic notes . . . . . . . . . . . . . . . . . . 9

1.5 Notational conventions . . . . . . . . . . . . . . . . . 10

$\begin{array}{ll}\text { I Background } & 11\end{array}$

2 Complexity 13

2.1 Models of computation . . . . . . . . . . . . . . . . . . 14

2.1 .1 Turing machines ... . . . . . . . . . . . . . 14

2.1.2 Non-deterministic Turing machines . . . . . . . . . . . 16

2.1.3 RAM machines . . . . . . . . . . . . . . . 16 
2.1.4 Minsky machines . . . . . . . . . . . . . . . . 18

2.1.5 RASP machines . . . . . . . . . . . . . . . . . . . 19

2.2 Computability . . . . . . . . . . . . . . . . . . . . 19

2.2.1 Church-Turing thesis . . . . . . . . . . . . . . 19

2.2.2 Turing-completeness . . . . . . . . . . . . . 20

2.3 Computational complexity theory . . . . . . . . . . . . . 21

2.3.1 Time complexity ................. 22

2.3.2 Space complexity ................. . . . 22

2.3.3 Asymptotic complexity and big O notation . . . . . . . 23

2.3.4 Amortized complexity analysis ... . . . . . . . . 24

2.3.5 Relation between RAM machines and Turing machines . . . 25

2.3.6 Complexity classes . . . . . . . . . . . . . 26

3 Constraint Handling Rules $\quad 27$

3.1 Syntax and semantics of CHR . . . . . . . . . . . . . . . 28

3.1 .1 Syntax . . . . . . . . . . . . . . . . 28

3.1.2 Semantics: informal introduction by example . . . . . . . 30

3.1.3 Logical semantics . . . . . . . . . . . . . . . . . 32

3.1.4 Abstract operational semantics $\omega_{t} \ldots \ldots . \ldots 33$

3.1.5 Derivations ................... . . 34

3.2 Program properties . . . . . . . . . . . . . . 36

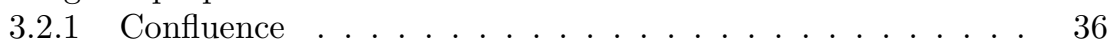

3.2.2 Termination . . . . . . . . . . . . . . 37

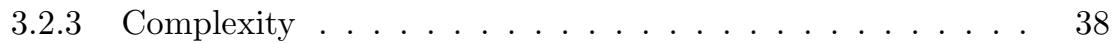

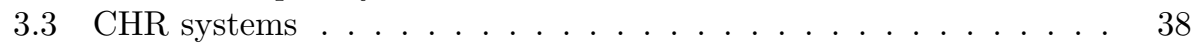

$3.3 .1 \quad \mathrm{CHR}(\mathrm{LP}) \ldots \ldots \ldots \ldots \ldots$

$3.3 .2 \mathrm{CHR}(\mathrm{FP}) \ldots \ldots \ldots \ldots$

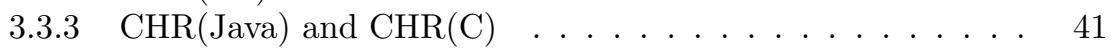

3.3.4 The Leuven CHR system . . . . . . . . . . . . . . . . 41

3.4 Extensions of CHR . . . . . . . . . . . . . . . . . . . . . . . 42

3.4.1 Disjunction and search . . . . . . . . . . . . . 42

3.4.2 Negation and aggregates ................ . . . . . . . . 42

3.4.3 Adaptive CHR . . . . . . . . . . . . . . . . . . . . 43

3.4 .4 Solver hierarchies . . . . . . . . . . . . . . . . . 43

3.5 Example CHR programs . . . . . . . . . . . . . . . . 44

3.5.1 Programming in CHR, in practice . . . . . . . . . . 44

3.5.2 Fibonacci numbers . . . . . . . . . . . . . . . . 46

3.5.3 Zebra puzzle .................... 46

3.5.4 Sudoku puzzle solver . . . . . . . . . . . . . . . . . . 49

3.6 Applications of CHR . . . . . . . . . . . . . . . . 50

3.6.1 Constraint solvers ................. 51

3.6.2 Automatic solver generation . . . . . . . . . . . . . 52

3.6.3 Type systems ................. 53 


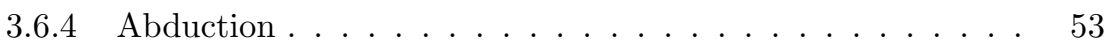

3.6.5 Computational linguistics . . . . . . . . . . . . . . 53

3.6.6 Testing and verification ............... . . . . 54

3.7 Related formalisms . . . . . . . . . . . . . . . . . . 54

3.7 .1 Join-Calculus . . . . . . . . . . . . . . . . . . 55

3.7 .2 Logical Algorithms . . . . . . . . . . . . . . . . . 55

3.7.3 Graph Transformation Systems . . . . . . . . . . . 56

3.7 .4 Petri nets . . . . . . . . . . . . . 56

4 Compilation of CHR $\quad 57$

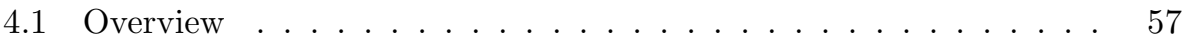

4.1.1 Standard compilation scheme . . . . . . . . . . . 58

4.1.2 Schemes based on the standard compilation scheme . . . . 59

4.1.3 Other compilation schemes . . . . . . . . . . . . 59

4.1.4 CHR with rule priorities . . . . . . . . . . . . . 60

4.2 Implementing the standard scheme . . . . . . . . . . . . . . . . 60

4.2.1 Constraint representation ... . . . . . . . . . . . 62

4.2.2 Some implementation details . . . . . . . . . . . . . . 64

4.3 Refined operational semantics $\omega_{r} \ldots \ldots \ldots$. . . . . . . . . 64

4.3.1 Definition of the $\omega_{r}$ semantics . . . . . . . . . . . 65

4.3.2 Passive occurrences . . . . . . . . . . . . . . 66

4.3.3 Call-based refined operational semantics $\omega_{c} \ldots \ldots$. . . . 69

4.4 Optimizations ....................... 69

4.4.1 Propagation history maintenance . . . . . . . . . . . 69

4.4 .2 Late storage . . . . . . . . . . . . . . . . . . . . . . . 71

4.4.3 Never-stored constraints . . . . . . . . . . . . . . . 72

4.4.4 Functional dependencies . . . . . . . . . . . . . 72

4.4.5 Other optimizations based on abstract interpretation . . . . 73

4.4.6 Indexing and join ordering . . . . . . . . . . . . 73

5 Implementing Classic Algorithms in CHR $\quad 75$

5.1 The union-find algorithm . . . . . . . . . . . . 76

5.2 Dijkstra's shortest path algorithm . . . . . . . . . . . . 77

5.2.1 The single-source shortest path problem . . . . . . . . . 77

5.2 .2 Dijkstra's algorithm . . . . . . . . . . . . . 78

5.2.3 Fibonacci heaps .................. 80

5.2 .4 Time complexity ................. 83

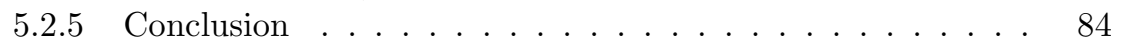

5.3 Hopcroft's algorithm for finite automata . . . . . . . . . . . 86

5.3 .1 Minimizing finite automata . . . . . . . . . . 86

5.3.2 Hopcroft's algorithm in CHR ............. 87

5.4 Discussion and conclusion . . . . . . . . . . . . . . . 89 
II Optimizing Compilation of CHR

6 Guard Reasoning $\quad 93$

6.1 Introduction . . . . . . . . . . . . . . . . 93

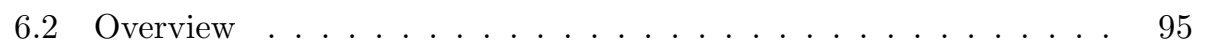

6.2.1 Guard simplification . . . . . . . . . . . . . . 95

6.2.2 Head matching simplification . . . . . . . . . . . . . 97

6.2.3 Type and mode declarations . . . . . . . . . . . . . . 97

6.2.4 Domain knowledge declarations . . . . . . . . . . . . . 97

6.2.5 Occurrence subsumption . . . . . . . . . . . . . . 98

6.3 Formalization . . . . . . . . . . . . . . . . . . . 100

6.3.1 Implicit preconditions . . . . . . . . . . . . . 100

6.3.2 Correctness of guard simplification . . . . . . . . . . . 101

6.3.3 Correctness of optimizing subsumed occurrences . . . . . . 105

6.4 Implementation of guard reasoning . . . . . . . . . . . . . . . . . 107

6.4.1 Entailment checking . . . . . . . . . . . . . . 107

6.4 .2 Inferring information . . . . . . . . . . . . . . 111

6.4.3 Using the information . . . . . . . . . . . . . . 112

6.5 Experimental results . . . . . . . . . . . . . . . . 112

6.5.1 Generated code comparison . . . . . . . . . . . . . . 112

6.5.2 Guard simplification results . . . . . . . . . . . . . 114

6.5.3 Occurrence subsumption results . . . . . . . . . . . 116

6.6 Guard reasoning under the $\omega_{p}$ semantics . . . . . . . . . . . . . . 116

6.7 Conclusion . . . . . . . . . . . . . . . . . 118

6.7 .1 Related work ................... 118

6.7.2 Future work. . . . . . . . . . . . . . 119

7 Indexing Techniques $\quad 121$

7.1 Attributed variables . . . . . . . . . . . . . . . . . 122

7.2 Hash tables . . . . . . . . . . . . . . . . . . 122

7.2 .1 Ground hash tables . . . . . . . . . . . . . 123

7.2 .2 Non-ground hash tables . . . . . . . . . . . . . . . 124

7.3 Arrays . . . . . . . . . . . . . . . . . . . 125

7.4 Experimental evaluation . . . . . . . . . . . . . . 125

7.5 Other possibilities for indexing . . . . . . . . . 126

8 Memory Reuse 129

8.1 Examples and basic ideas . . . . . . . . . . . . . . . . . 129

8.1 .1 In-place updates . . . . . . . . . . . . . . . . . . . . . . . . . . . . . . . . . . . 130

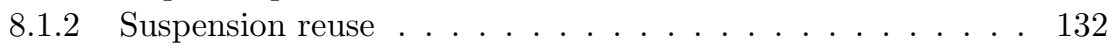

8.2 Formal framework . . . . . . . . . . . . . . . . . 132

8.2.1 Extended call-based refined operational semantics $\omega_{c}^{\prime}$. . . 134

8.2 .2 Equivalence of $\omega_{c}$ and $\omega_{c}^{\prime} \ldots \ldots \ldots 134$ 
8.2.3 Defining the optimizations . . . . . . . . . . . . . . 135

8.3 Implementation of memory reuse . . . . . . . . . . . . . . . . . . 136

8.3.1 Constraint representation . . . . . . . . . . . . . 136

8.3 .2 Inlining and specialization . . . . . . . . . . . . . . 137

8.3 .3 Suspension reuse . . . . . . . . . . . . . . . 137

8.3 .4 In-place updates . . . . . . . . . . . . . . . . . . 138

8.3.5 Interference with late storage . . . . . . . . . . . . . 139

8.4 Experimental results . . . . . . . . . . . . . . . . . 140

8.5 Conclusion ....................... . . 141

8.5.1 Related work . . . . . . . . . . . . . . . . 142

8.5 .2 Future work . . . . . . . . . . . . . . . . 142

9 Join Ordering 143

9.1 Motivation and approach ................. 144

9.1 .1 Processing trees . . . . . . . . . . . . . . . . 144

9.1.2 Static and dynamic join ordering . . . . . . . . . . . . 146

9.2 Cost model . . . . . . . . . . . . . . . . . . . . . . . . 147

9.2.1 Notation . . . . . . . . . . . . . . . . . . 148

9.2 .2 Partial joins . . . . . . . . . . . . . . . . . . 149

9.2.3 Cost formula . . . . . . . . . . . . . . . . . . 149

9.3 Approximating costs . . . . . . . . . . . . . . 150

9.3.1 Static cost approximations . . . . . . . . . . . . 150

9.3.2 Dynamic cost approximations . . . . . . . . . . . . . 152

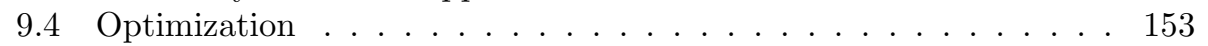

9.4 .1 Join graphs . . . . . . . . . . . . . . . . . 154

9.4 .2 Acyclic join graphs . . . . . . . . . . . . . . . . 154

9.4 .3 Cyclic join graphs . . . . . . . . . . . . . . . 157

9.4.4 Optimization cost versus join cost . . . . . . . . . . . 159

9.5 Discussion . . . . . . . . . . . . . . . . . . . . 159

9.5.1 First-few answers . . . . . . . . . . . . . . . . 159

9.5.2 Cyclic and acyclic join graphs . . . . . . . . . . . . 160

9.5.3 Join ordering in current CHR implementations . . . . . . . 162

9.5.4 Future work . . . . . . . . . . . . . . 162

III Computability and Complexity of CHR 163

10 CHR Machines 165

10.1 Definition of CHR machines . . . . . . . . . . . . . . . 166

10.2 CHR machines are Turing-complete . . . . . . . . . . . . . 167

10.3 Turing-complete subsets of CHR . . . . . . . . . . . . . 170

10.3.1 Only propagation rules . . . . . . . . . . . . . 170

10.3.2 No rule guards . . . . . . . . . . . . . . . 171 
10.3 .3 Only one rule . . . . . . . . . . . . . . . . . . . . 172

10.3.4 Only single-headed rules . . . . . . . . . . . . . . . . . 174

10.3.5 Propositional CHR . . . . . . . . . . . . . . . . . 177

10.3.6 Summary . . . . . . . . . . . . . . . . . . 178

10.4 Complexity of CHR machines . . . . . . . . . . . . . . . . . 180

10.4.1 Time complexity . . . . . . . . . . . . . . . . 180

10.4 .2 Space complexity . . . . . . . . . . . . . . . . . 181

11 Complexity-wise Completeness 183

11.1 RAM machine simulators . . . . . . . . . . . . . . . . . . . 184

11.2 Complexity meta-theorem . . . . . . . . . . . . . . . 185

11.2.1 CHR is Turing-equivalent . . . . . . . . . . . . . 185

11.2.2 Complexity of the compiled code . . . . . . . . . . . 186

11.2.3 Complexity meta-theorem . . . . . . . . . . . . . . 190

11.3 Complexity-wise completeness . . . . . . . . . . . . . . . . 191

11.3.1 Discussion . . . . . . . . . . . . . . . . . . . 192

11.3.2 Register initialization . . . . . . . . . . . . . . . 192

11.3.3 Related and future work . . . . . . . . . . . . . . . . 193

11.4 Other declarative languages . . . . . . . . . . . . . . . . . . . 194

11.4.1 Sufficient ingredients . . . . . . . . . . . . . . . . 195

11.4.2 Arrays in declarative languages . . . . . . . . . . . . . . . . 196

11.4.3 Experimental results . . . . . . . . . . . . . . . . . . . 198

11.4 .4 Summary . . . . . . . . . . . . . . . . 199

11.5 Constant factors . . . . . . . . . . . . . . . . 200

11.5.1 Complexity-wise completeness in practice . . . . . . . . . 201

11.5.2 Experimental evaluation . . . . . . . . . . . . . . 202

11.5.3 Conclusion: $\mathrm{CHR}(\mathrm{hProlog}) / \mathrm{C} \approx 10 \ldots . . . . . . .204$

12 Generalized CHR Machines 205

12.1 Strategy classes . . . . . . . . . . . . . . . . . . . . . . . . . . 205

12.1.1 Execution strategies . . . . . . . . . . . . . 206

12.1.2 Strategy classes . . . . . . . . . . . . . . . . 207

12.1.3 Generalized confluence . . . . . . . . . . . . . . . . 209

12.2 General CHR machines . . . . . . . . . . . . . . . . . . 209

12.2.1 Terminology . . . . . . . . . . . . . . 211

12.2.2 Complexity of general CHR machines . . . . . . . . . . 211

12.3 Non-deterministic CHR machines . . . . . . . . . . . . . . . . . . 211

12.4 Self-modifying CHR machines . . . . . . . . . . . . . . . 213

12.4.1 Definition ................... . . 214

12.4.2 Complexity of CHRSP machines . . . . . . . . . . . . 215

12.5 Summary and conclusion . . . . . . . . . . . . . 216

12.5.1 Complexity summary . . . . . . . . . . . . . 216

12.5.2 Future work . . . . . . . . . . . . . 216 
13 Conclusion $\quad \mathbf{2 1 7}$

13.1 Contributions . . . . . . . . . . . . . . . . . . 217

13.2 Future work . . . . . . . . . . . . . . . . . 218

13.2.1 Grand challenges for CHR . . . . . . . . . . . . . 219

13.2.2 Beyond CHR . . . . . . . . . . . . . . . 220

A RAM Machine Simulators $\quad 221$

B Benchmark Results $\quad 225$

Bibliography 231

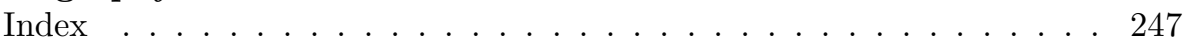

List of Symbols . . . . . . . . . . . . . . . . . . . . . . . . . . . . . . . . . . . . . . . . . . . . . . .

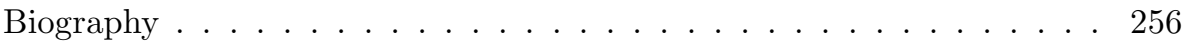

List of Publications . . . . . . . . . . . . . . . . . . . 257

Nederlandstalige Samenvatting $\quad$ NL 1

Hoofdstuk 1: Inleiding . . . . . . . . . . . . . NL 5

Deel I - Achtergrond . . . . . . . . . . . . . . . . . . NL 8

Hoofdstuk 2: Complexiteitstheorie . . . . . . . . . . NL 8

Hoofdstuk 3: Beperkingsafhandelingsregels (CHR) . . . . . . . NL 9

Hoofdstuk 4: Compilatie van CHR . . . . . . . . . . . . . NL 11

Hoofdstuk 5: Implementatie van klassieke algoritmes . . . . . . . . NL 11

Deel II — Optimaliserende CHR-compilatie . . . . . . . . . . . . . . . . NL 11

Hoofdstuk 6: Redeneren over regelcondities . . . . . . . . . . NL 11

Hoofdstuk 7: Indexeringstechnieken . . . . . . . . . . . . . NL 12

Hoofdstuk 8: Geheugenhergebruik . . . . . . . . . . . . NL 12

Hoofdstuk 9: Samenvoegingsvolgordeverbetering . . . . . . . . . NL 13

Deel III — Berekenbaarheid en complexiteit . . . . . . . . . . . . . . . . NL 13

Hoofdstuk 10: CHR-machines . . . . . . . . . . . . . . . NL 13

Hoofdstuk 11: Complexiteitsgewijze volledigheid . . . . . . . . . NL 13

Hoofdstuk 12: Veralgemeende CHR-machines . . . . . . . . . . . NL 14

Hoofdstuk 13: Besluit . . . . . . . . . . . . . . . . . . . . NL 14 


\section{List of Tables}

2.1 Instruction set of the RAM machine . . . . . . . . . . . . . . 17

2.2 Common asymptotic complexities . . . . . . . . . . . . 24

4.1 Meaning of the constraint suspension fields . . . . . . . . . . . . 64

7.1 Different detail levels in mode/type declarations . . . . . . . . . . 126

8.1 Meaning of the MState field in constraint suspensions . . . . . . . 136

9.1 Number of rules with $n$ heads (with cyclic join graphs) . . . . . . 160

11.1 Language classification in terms of complexity-wise completeness . 200

B.1 Benchmarks for guard simplification . . . . . . . . . . . 226

B.2 Benchmarks for occurrence subsumption . . . . . . . . . . . . 227

B.3 Benchmarks for suspension reuse and in-place updates . . . . . . . 228

B.4 Example RAM simulator queries used for benchmarking . . . . . . 229

B.5 Benchmarks for several RAM simulator implementations . . . . . 229

B.6 Benchmarks for the union-find algorithm, in different systems . . 230

B.7 Benchmarks for Dijkstra's algorithm, in different systems . . . . . 230

NL.1 Vertaling van Engelstalige terminologie . . . . . . . . . . . . . . . . NL 4 


\section{List of Figures}

1.1 Chapter dependencies .................. 8

2.1 Letter from Kurt Gödel to John von Neumann, 20 March 1956 . . 21

3.1 Transitions of the abstract (theoretical) operational semantics $\omega_{t} \quad$. 34

3.2 Timeline of CHR systems . . . . . . . . . . . . . . . 40

3.3 Sudoku board layout with 283576 solutions. . . . . . . . . . . . 50

4.1 Transitions of the priority semantics $\omega_{p} \ldots \ldots \ldots$. . . . . . . 61

4.2 Compilation scheme for a constraint occurrence . . . . . . . . . 63

4.3 Transitions of the refined operational semantics $\omega_{r} \ldots \ldots$. . . . . 66

4.4 Transitions of the call-based refined operational semantics $\omega_{c}$. . . 70

5.1 Pseudo-code description of Hopcroft's algorithm . . . . . . . . . 88

7.1 Runtimes for the union-find algorithm, with different indexes . . . 127

7.2 The data of the previous plot, on log-log scales . . . . . . . . . . 127

7.3 The same data, with runtime divided by reference complexity . . . 128

8.1 Constraint deletion followed by insertion, without optimizations . 131

8.2 In-place update . . . . . . . . . . . . . . . . . . 131

8.3 Constraint deletion followed by insertion, with suspension reuse . 133

8.4 Additional transitions of the extended call-based semantics $\omega_{c}^{\prime}$. . . 134

9.1 A left-deep processing tree for Example 30 . . . . . . . . . . . . 145

9.2 A bushy processing tree for Example 31 . . . . . . . . . . . . 146

9.3 Cyclic and acyclic join graphs . . . . . . . . . . . . . . . 154

9.4 KBZ join ordering algorithm for acyclic join graphs . . . . . . . . . 156

9.5 Join graph and ranks for Example 34 . . . . . . . . . . . . . . 156

9.6 Eliminating a clique in a join graph . . . . . . . . . . . . . . . 161

10.1 Simulation of Turing machines on a CHR machine . . . . . . . . . 169 
10.2 States of $\mathcal{M}_{\mathrm{TM}}$ and corresponding Turing machine states . . . . . 169

10.3 Tape representation for TMSIM-2R . . . . . . . . . . . . . . . . 173

10.4 Right move in TMSIM-2R, normal case . . . . . . . . . . . . . . . 173

10.5 Right move in TMSIM-2R, at the tape end . . . . . . . . . . . . 173

10.6 Overview of subsets of CHR and their Turing-completeness . . . . 179

10.7 Solver program for some crossword puzzle. . . . . . . . . . . . . . 181

11.1 Relationships between Turing, RAM, and CHR machines . . . . . 193

11.2 Results of the "NLoop" benchmark (cf. Table B.5) . . . . . . . . . 199

11.3 Assembler code and corresponding RAM machine query . . . . . . 201

11.4 Results of the "Union-find" benchmark (cf. Table B.6) . . . . . . . 203

11.5 Results of the "Dijkstra" benchmark (cf. Table B.7) . . . . . . . . 203

12.1 Execution strategies _. . . . . . . . . . . . . . . . . 210

12.2 Generalized confluence . . . . . . . . . . . . . . . . . 210

12.3 Example encoding of a CHR program for CHRSP machines . . . . 214

12.4 Modified Apply transition in the $\omega_{t}^{s p}$ semantics . . . . . . . . . . . 214 


\section{List of Listings}

1.1 Example Prolog program ................ . . 3

3.1 LEQ: Solver for the less-than-or-equal constraint . . . . . . . . . 31

3.2 PRIMES: Prime number generator . . . . . . . . . . . . . . 31

3.3 SUM: Sum of the elements of a list . . . . . . . . . . . . 45

3.4 FIBONACCI: Top-down computation of Fibonacci numbers . . . . . 46

3.5 ZEBRA: Solver for puzzles like the zebra puzzle . . . . . . . . . . 47

3.6 Formulation of the zebra puzzle . . . . . . . . . . . . . . . . 48

3.7 SUDOKU: Solver for Sudoku puzzles . . . . . . . . . . . . 49

4.1 P-DIJKSTRA: Dijkstra's shortest path algorithm in $\mathrm{CHR}^{\mathrm{rp}} \ldots . . .60$

5.1 N-UNION-FIND: Naive implementation of the union-find algorithm 76

5.2 UNION-FIND: Optimal implementation of the union-find algorithm 77

5.3 DIJKSTRA: Dijkstra's shortest path algorithm . . . . . . . . . . . 79

5.4 FIB-HEAP: Fibonacci-heap implementation of priority queues . . . 82

5.5 HOPCROFT: Hopcroft's algorithm for minimizing finite automata . 88

6.1 Entailment checker: conversion of the antecedent to normal form . 108

6.2 Entailment checker: propagation of known/1 constraints . . . . . . 109

6.3 Entailment checker: part of the rest of the program . . . . . . . . . 109

10.1 TMSIM: Turing machine simulator . . . . . . . . . . . . . . . . 167

10.2 TMSIM-PROP: Turing machine simulator, only propagation rules . 171

10.3 TMSIM-NG: Turing machine simulator, without rule guards . . . . 172

10.4 TMSIM-2R: Turing machine simulator in two rules . . . . . . . . 173

10.5 TMSIM-2RD: A version of TMSIM-2R with double adj/3 constraints 174

10.6 TMSIM-1R: Turing machine simulator in one rule . . . . . . . . . . 175

10.7 MINSKY-A: Minsky machine encoding using argument arithmetic . 175

10.8 MINSKY-C: Minsky machine encoding using constraint chains . . . 176

10.9 MINSKY-P: Minsky machine encoding in propositional refined CHR 177 
11.1 Alternative register initialization, using the $\omega_{r}$ semantics . . . . . . 193

12.1 3SAT: NCHR program to solve 3SAT clauses . . . . . . . . . . . . 212

12.2 HAMILTON: CHRSP program to find Hamiltonian paths . . . . . . 215

A.1 PARAMSIM: Simulator of Peano-arithmetic RAM machines . . . . 221

A.2 RAMSIMUL: Simulator of standard RAM machines . . . . . . . . . 221

A.3 Simulator of RAM machines, written in pure Prolog . . . . . . . . 222

A.4 Simulator of RAM machines, written in Haskell . . . . . . . . . . . 222

A.5 Simulator of RAM machines, written in Maude . . . . . . . . . . . 223

A.6 More efficient RAM simulator in Maude, using Map . . . . . . . . . 223

A.7 Simulator of RAM machines, written in Jess . . . . . . . . . . . . . 224 



\title{
Preface
}

\begin{abstract}
"There are many different styles of composition. I characterize them always as Mozart versus Beethoven. When Mozart began to write, he had the composition ready in his mind. He wrote the manuscript and it was 'aus einem Guss' (casted as one). And it was also written very beautifully. Beethoven was an indecisive tinkerer: he began to write before he had the composition ready and plastered parts over to change them. There was a certain place where he plastered over nine times; one has removed the layers carefully to see what had happened and it turned out the last version was the same as the first one."
\end{abstract}

— Edsger Dijkstra (1930-2002)

Since the preface is probably the only chapter that is actually read by a significant proportion of the target audience of this genre of literature, while it is also the only chapter not subjected to the full scrutiny of the academic jury, I will take the liberty to throw in some statements that are slightly controversial or smug, or overly solemn and perhaps even pompous, as exemplified by this opening sentence, and probably even more so by the next one.

Sparks of inspiration, which bend or fork lines of research in new directions, usually originate in surprisingly modest questions and seemingly minor practical problems. Their true significance is often only understood in retrospect.

Consider, as a typical example, the story of how CHR research started in Leuven. Bart Demoen had implemented a new feature in hProlog, called attributed variables. He wanted to try it out, so he asked his Ph.D. student Tom Schrijvers to implement a CHR system in hProlog for the purpose of benchmarking the performance of attributed variables. This hProlog CHR system was based on the SICStus CHR implementation by Christian Holzbaur, which used SICStus' attributed variables. What started as a small 'benchmark' project soon became the central topic of Tom's Ph.D. thesis and lead to the development of the Leuven CHR system and many novel analysis and optimization techniques for CHR.

Or consider the chain of events triggered by one small question of Bart at Tom's Ph.D. defense. Tom had presented an optimal implementation in CHR, 
by Thom Frühwirth and himself, of the legendary union-find algorithm - the algorithm which takes just a tiny bit more than linear time, the tiny bit being the inverse Ackermann function, which grows so incredibly slowly that, modulo pedantry, it can be treated as a fancy disguise for a constant. Anyway, at the end of the presentation, Bart asked the following innocent-sounding question: "You have showed that the union-find algorithm can be implemented with optimal complexity in CHR; could you do the same for other algorithms? Perhaps for any other algorithm? Could you, for instance, simulate any Turing machine in CHR?"

I do not remember Tom's answer, but some days later we were investigating the question together. It was about a month before the submission deadline for the second CHR workshop, which was collocated with ICLP 2005 in Sitges, Spain. Somewhat hurriedly we wrote down our findings, which consisted mostly of handwaving 'proof sketches' that were supposed to convince the reader of an affirmative answer to Bart's question. Apparently the handwaving was sufficiently vigorous, since the resulting paper received a Best Paper Award. However, when we later tried to formalize the results and experimentally validate them, we soon experienced some 'minor technical problems' which had to be solved first, leading to a number of new compiler optimizations and, eventually, resulting in the thesis you're holding in your hands, or reading on your computer screen, right now.

Although not yet based on a very solid theoretical foundation, at the end of that CHR workshop, everybody was convinced of our conjecture that everything can indeed be done in CHR with the optimal complexity. Or so we thought. At next year's ICLP 2006 in Seattle, during one of the coffee breaks, Agostino Dovier approached Bart and me. He told us that while he believed our result to be valid, he would only fully be convinced after we could show him an implementation of John Hopcroft's DFA minimization algorithm in CHR, for he had been struggling himself to implement that particular algorithm (in another programming language). To his and my own relief, I managed to meet his challenge; the resulting CHR program is published for the first time in this Ph.D. thesis.

Computer programming is like composing music: on the surface, it is about writing down detailed instructions (for musicians or for processors), but essentially, it is about elegance and beauty. Both are said to require a mathematically inclined mind. And in both cases, the bulk of the contemporary mainstream manifestations are uninspired, repetitive, and sold overpriced by monopolistic companies. But I'm digressing. In Edsger Dijkstra's classification scheme for compositions (see the quote starting this preface), I tend to linger at the Beethoven side of the spectrum when writing programs or text. Although modern document preparation systems like $\mathrm{AT}_{\mathrm{E}} \mathrm{X}$ have obsoleted the technique of 'plastering over' - thanks, Donald Knuth and Leslie Lamport! (and the numerous IATEX package authors) - CVS records will confirm that occasionally I too have modified CHR rules repeatedly, or 
changed the structure of a text over and over again, only to end up exactly where I started. More often though, after many iterations of refinement, the end result was even worse than the crude initial attempt. It is only a small consolation that I am probably not the only one to envy those who, like Mozart, are able to produce ingenious programs and papers, seemingly effortlessly and 'aus einem Guss'.

I have been very lucky to have had two mentors/colleagues/coauthors, who just like Dijkstra and Mozart appear to possess this remarkable ability to almost instantaneously deliver work of the most outstanding quality. The first one is my supervisor, Bart Demoen, who was always available when I needed his help, while he never disturbed me when I did not. I can honestly say that I cannot imagine a wiser, cleverer, more uplifting, more caring, or more approachable supervisor. My second mentor was Tom Schrijvers, who was a reliable guide in the strange realm of CHR, especially in my first years as a Ph.D. student. Without Tom, I would probably still be lost in the tangled jungle of the Leuven CHR system, except of course that without Tom there would be no Leuven CHR system.

Two other coauthors and members of the Leuven CHR team share an office with me: Leslie De Koninck and Peter Van Weert. Leslie is probably going to defend his Ph.D. just a bit earlier than me, although he started a full year after me. He can write papers faster than I can read them, and I'm not a particularly slow reader. I often tried to slow him down by suggesting a break, but my strategy proved ineffective because we then invariably went to get a cup of coffee, which of course did not help to reduce Leslie's productivity. In contrast, Peter is a perfectionist who just like Beethoven likes to take his time to fiddle and tinker until everything is precisely as it has to be. I have tremendously enjoyed and benefited from the collaboration with both of them.

I have not yet had the chance to (formally) collaborate with the peripheral Leuven CHR team members: the 'terminators' Paolo Pilozzi and Dean Voets, the 'educator' Gerda Janssens, and the 'accelerator' Pieter Wuille, the implementer of the fastest CHR system, in C. They all contribute significantly to the inspiring atmosphere permeating the 200A building.

Incidentally, in the last few years, this beloved building has been permeated by so much more than only the usual inspiring atmospheres: during the construction works - two floors were added on top of the building - it was regularly permeated by additional atmospheres of the noisy or dusty kind, or by water, leaking or flowing through cracks or holes in the unfinished roof, causing occasional electricity outages. With an impeccable feeling for timing, my partner and I have renovated our own little house in the very same period, making sure that when attempting to work from home, the somewhat suboptimal working conditions of the department building were accurately mirrored. But I'm digressing, again.

The Leuven CHR team is part of the DTAI research group, which is headed by Maurice Bruynooghe. Maurice is not just a pioneer of Logic Programming, he is also a pioneer of Open Access publishing. In November 1999, after long but 
fruitless negotiations with Elsevier for cheaper subscription prices, the entire 50person editorial board of the Journal of Logic Programming, including its editorin-chief, Maurice, collectively resigned and created a new journal, Theory and Practice of Logic Programming, published by Cambridge University Press at a fraction of Elsevier's price, and publicly available at the arXiv Computing Research Repository (CoRR). I admire his punctuality and insistence in reminding all DTAI members to make their work available in Open Access repositories.

Besides the persons already mentioned above, I would like to thank my coauthors - Bart, Tom, Leslie, Peter, Thom Frühwirth, Danny De Schreye, and Joost Vennekens; the members of the jury — Thom, François Fages, Maurice, Bart, Frank Piessens, and Paul Van Houtte; and the anonymous referees I obviously do not know their names. I also thank all current and past members of DTAI and the department in general, including of course the secretaries, in particular, Denise Brams and Karin Michiels. Having been the system group representative for DTAI for some years, I have very much enjoyed working together with the system group people: Jean Huens, Bart Swennen, Kris Wessels, Anita Ceulemans, Liliane De Roose, and Kris Vangeneugden, and the other research group representatives. Finally I thank my colleagues during stays abroad: Peter Stuckey, Gregory Duck, and the others in Melbourne, and Thom, Hariolf Betz, Ingi Sobhi, Frank Raiser, and the others in Ulm.

From August 2004 until December 2005 I was supported by the Research Foundation - Flanders (FWO-Vlaanderen) through projects G.0144.03 and G.0160.02. Since January 2006, I was funded by a Ph.D. grant of the Institute for the Promotion of Innovation through Science and Technology in Flanders (IWT-Vlaanderen). Obviously, I am much indebted to these two funding agencies, FWO and IWT, and I hope the latter will forgive me for my attempts to make them adopt the better wage conditions of the former; I wholeheartedly thank everybody who has helped me in these attempts, in particular, the 1450 signers of the petition.

Penultimately, I would like to express my admiration and gratitude towards Alan Turing for inventing computer science and AI, Richard Stallman for the GNU project and the Free Software movement, and, like Noam Chomsky, for showing that computer scientists can also be political activists, Karl Marx for inventing socialism and Leon Trotsky for attempting to guard it from Stalinist deformations, Douglas Adams and Bill Bryson for writing extremely funny books and Douglas Hofstadter, John D. Barrow, Richard Dawkins, and Jared Diamond for writing extremely interesting books, and Ludwig van Beethoven for composing magnificently beautiful music, plastered or not.

Finally, I would thank my family and friends. In particular, I thank my parents for everything they have done for me, and my partner Tina De Greef for always encouraging me, passionately and enthusiastically. 


\title{
Chapter 1
}

\section{Introduction}

\begin{abstract}
"Constraint programming represents one of the closest approaches computer science has yet made to the Holy Grail of programming: the user states the problem, the computer solves it."
\end{abstract}

— Eugene Freuder (1945-)

This first chapter introduces the research presented in this dissertation and provides information about the text itself. Section 1.1 puts Constraint Handling Rules in a broader context by sketching its prehistory. Then, in Section 1.2, we state the main research goals that lead to our contributions. Section 1.3 gives an overview of the structure of the text. In Section 1.4 we refer to several publications that are incorporated in this text. Finally, Section 1.5 introduces the notational conventions that are used in the remainder of the text.

\subsection{Context}

Early computers were programmed using binary machine code. Programming was an extremely tedious and error-prone process, in which the programmer had to remember numeric machine codes and had to calculate memory addresses manually. In the early 1950s the first assembly languages were developed, based on somewhat more human-readable abbreviations for the elementary instructions and symbolic instruction labels. We describe such languages as being very low-level, that is, very close to the hardware on which the programs are executed. Translating assembly programs to machine code is rather straightforward; a program that automates this translation is called an assembler. Note that every CPU architecture has its own assembly language, since the assembly mnemonics directly correspond to particular CPU instructions.

In order to improve programming productivity, higher level programming languages were developed. Early examples of such languages are FORTRAN (1954), 
ALGOL (1958), COBOL (1959), APL (1962), BASIC (1964), Pascal (1970), and C (1972). These programming languages are usually translated to assembly languages; the translation program is called a compiler. Nowadays we describe these languages as being low-level, but in their days they were called high-level programming languages. One of the main advantages of these programming languages was the ability to run the same program on machines with a different architecture as soon as a compiler was written for a particular platform, all programs can be compiled and executed on that platform. As a result, a program that is written in $\mathrm{C}$ in the 1970s can still be executed on a modern computer, although the underlying hardware has of course significantly changed.

\subsubsection{Declarative programming}

These early programming languages were imperative languages: a program is essentially a sequence of processing steps, describing an algorithm that solves some problem. In higher-level languages, the steps are larger - more details are hidden from the programmer and taken care of by the compiler - in order for the programmer to see the overall picture more easily. However, in all imperative languages, a program has to describe how to solve the problem.

The ideal of declarative programming is that the program describes what the problem is, not how to solve it. Instead of having to come up with an algorithm to find a solution, the programmer merely has to describe the desired properties of a solution - the compiler translates this specification to executable code that searches for the solution. Clearly, for declarative programming languages, the task of the compiler is much more complicated than for imperative languages.

Declarative programming languages are often classified into three categories:

Functional programming languages are based on the evaluation of mathematical functions. Examples are LISP (1958), ML (1973), Erlang (1987), and Haskell (1990).

Logic programming languages are based on mathematical logic, where theorem proving or model generation provides the execution mechanism. Most logic programming languages are based on Prolog (1972), developed by Alain Colmerauer in Marseille and Robert Kowalski in Edinburgh.

Constraint programming languages are based on the concept of imposing various kinds of constraints on candidate solutions. Examples are ILOG CP (1993) and Gecode (2005).

At the intersection of these paradigms are a multitude of hybrid languages. For example, Mercury (1995) and Curry (1996) are functional logic programming languages, CHIP (1985) and $\mathrm{ECL}^{i} \mathrm{PS}^{e}$ (1992) are constraint logic programming languages, and $\mathrm{Oz} /$ Mozart (1991) combines all three paradigms. 
Listing 1.1: Example Prolog program

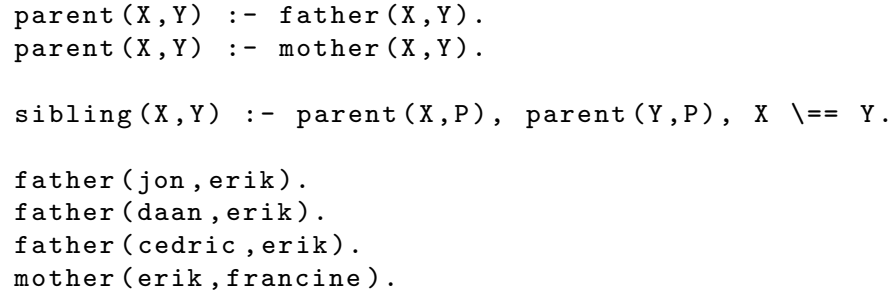

\subsubsection{Constraint logic programming}

Constraint Logic Programming (CLP) combines the paradigms of logic programming and constraint programming. We very briefly introduce these two paradigms.

\section{Logic programming}

Logic programs consist of logical formulae that define logical predicates. In Prolog, the formulae are definite Horn clauses, which are of the form $h \leftarrow b_{1} \wedge \ldots \wedge b_{n}$, that is, they are implications where the antecedent is a conjunction of atoms and the consequent is a single atom. The actual Prolog syntax uses the symbol ":-" to denote " $\longleftarrow$ " and commas to separate conjuncts, so the general form of a Prolog clause is $\mathrm{h}:-\mathrm{b}_{1}, \ldots, \mathrm{b}_{n}$. If the antecedent is the trivial empty conjunction (also denoted as true), it may be omitted. Such clauses are called facts.

Listing 1.1 shows a simple Prolog program. It defines the predicates parent $/ 2$, sibling $/ 2$, father $/ 2$, and mother $/ 2$, which can all be seen as binary relations. For example, "father(jon,erik)" is interpreted as "the father of jon is erik". The first clause states that if the father of $\mathrm{X}$ is $\mathrm{Y}$, then $\mathrm{Y}$ is also a parent of $\mathrm{X}$. The second clause states that $\mathrm{Y}$ is a parent of $\mathrm{X}$ if $\mathrm{Y}$ is the mother of $\mathrm{X}$. These clauses can be combined in a single clause using the disjunction operator ";" :

$$
\operatorname{parent}(\mathrm{X}, \mathrm{Y}):-\operatorname{father}(\mathrm{X}, \mathrm{Y}) ; \operatorname{mother}(\mathrm{X}, \mathrm{Y}) \text {. }
$$

The third clause can be read as follows: if $\mathrm{X}$ has a parent $\mathrm{P}$, and $\mathrm{Y}$ has the same parent $P$, and $X$ and $Y$ are not the same person ( $\backslash==$ " denotes syntactic inequality in Prolog), then $\mathrm{X}$ and $\mathrm{Y}$ are siblings.

To use a Prolog program, we enter a query (also called goal) which is then answered by the Prolog system. For example, if we ask whether daan and cedric are siblings, Prolog answers affirmatively:

?- sibling(daan, cedric).

yes 
If we ask whether jon and erik are siblings, we get a negative answer:

?- sibling (jon, erik).

no

The reason is that Prolog uses the closed-world assumption, i.e. it assumes that all facts are given so if it does not find a proof of the query, the query is assumed to be false. Because of the closed-world assumption, Prolog clauses may not be read as logical implications. Instead, the logical reading of a Prolog program is given by its Clark completion. Simply put, when a Prolog predicate is defined in a single clause, the arrow ":-" is read as "if and only if" instead of just "if".

We can also use variables in queries. These variables are implicitly existentially quantified; the Prolog system instantiates them to a value for which the query becomes true. There can be multiple solutions:

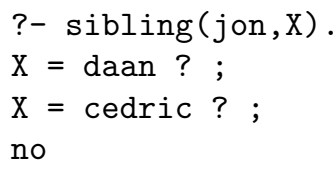

The "no" at the end indicates that there are no further solutions. An introduction to logic programming can be found in Clocksin and Mellish (1984); we refer the more advanced reader to O'Keefe (1990) and Bratko (2001).

\section{Constraint programming}

In constraint programming, built-in algorithms are used to generate solutions that respect given constraints. For example, in a constraint solver for integer domains, when given the constraints $A$ in 1.10 and $A * B \#=50$, we get the solutions $(A=1, B=50),(A=2, B=25),(A=5, B=10)$, and $(A=10, B=5)$.

Constraints often come in two flavors: ask and tell. When a constraint is imposed, that is, it is declared to hold, we call it a tell constraint - the constraint system is told that the constraint should be enforced. When we are wondering whether a constraint is implied by the constraints, we use ask constraints - we ask the constraint system whether or not this constraint is entailed. For example, suppose we have told the constraint X \#> 5 and nothing else. Afterwards, if we ask whether $\mathrm{X}>3$, the answer would be affirmative, but if we ask whether $\mathrm{X}>8$, the answer would be negative.

We refer the reader to Marriott and Stuckey (1998) and Frühwirth and Abdennadher (2003) for an introduction to constraint programming.

\section{Constraint logic programming}

CLP systems consist of a logic programming language extended with one or more constraint solvers. For example, a CLP system can be obtained by extending 
Prolog with a linear equation solver over the real numbers. For introductions to CLP, we refer the reader to Jaffar and Lassez (1987), Frühwirth et al. (1992), Jaffar and Maher (1994), and Jaffar et al. (1998).

Early CLP systems were based on the so-called "black-box" approach. Constraint solvers were implemented in efficient low-level programming languages, for example C. The resulting systems were efficient but not very flexible. It was very hard to modify or extend these constraint solvers, for example to add new types of constraints or different constraint domains.

Many so-called "glass-box" approaches have been proposed to obtain more customizable constraint systems; Frühwirth (1998) gives a brief overview. These approaches typically only allowed to extend a solver over a particular (usually finite) constraint domain. To directly implement custom constraint domains, only the approach of Holzbaur (1992) could be used, but this was tedious and has been described as "constraint assembler" programming (Frühwirth 1998).

\subsubsection{Constraint Handling Rules}

In the early 1990s, motivated by the lack of flexibility and customizability of the original "black-box" and "glass-box" constraint solvers, Thom Frühwirth (1992) designed a new special-purpose programming language (or rather, a language $e x$ tension), called Constraint Handling Rules. From its conception, CHR was meant to be used as a high-level and declarative language for implementing constraint solvers. As the name "Constraint Handling Rules" suggests, CHR programs consist of rules to handle user-defined constraints. The rules eventually reduce the user-defined constraints to built-in constraints. CHR is a language extension: it adds new functionality to an existing programming language, which is called the host language and which provides the built-in constraints. Historically, CHR was usually added to Prolog, but today there are also several CHR systems in other languages, like Java and Haskell.

As time went by, it became clear that CHR and its variants can be used for reasoning systems in general (not just constraint solvers), including deduction and abduction. In CHR, techniques like forward and backward chaining, bottom-up and top-down evaluation, integrity constraints, tabling and memoization can easily be implemented and combined (Holzbaur and Frühwirth 2000b).

More recently, CHR is seen as a concurrent very-high-level general-purpose programming language, especially suitable for rapid prototyping. Because CHR is such a high-level language, CHR programs are often more concise than pseudocode descriptions of the corresponding algorithms.

The growing scope of CHR - from a special-purpose language for implementing constraint solvers to a general-purpose language - was made possible by increasingly more efficient CHR systems. In turn, improving the performance of general-purpose CHR programs (for instance, a CHR implementation of the classic union-find algorithm) motivated the creation of more efficient CHR systems. 


\subsection{Goals and contributions}

In this dissertation we wish to demonstrate and enhance the usability of CHR as a high-level general-purpose programming language. Hence, our main research goal is to study and improve the performance of CHR systems. In order to study the performance (in terms of both runtime and memory usage), we need to analyze the computational complexity of the CHR language and its implementations. Clearly, this requires a thorough investigation of state-of-the-art CHR systems and of the compiler optimizations they employ. To improve the performance, we propose a number of new compiler optimizations. In both cases we are not only interested in the asymptotic complexities (i.e. scalability) but also in the constant factors (i.e. absolute performance).

It is clear that high-level languages have many advantages - shorter development time, more readable and adaptable code, etc. However, it is usually the case that low-level languages are more efficient. The central question of this research is the following: "what is the penalty, in terms of computability and/or complexity, of using the very-high-level language CHR instead of a low-level language, say, the language C ?". This is not a trivial question: some high-level languages (e.g. SQL) are not Turing-complete and thus come with a severe penalty in terms of computability; other high-level languages (e.g. pure Prolog) are Turing-complete but do not allow every algorithm to be implemented with the best known asymptotic time and space complexity (e.g. the union-find algorithm is not known to be efficiently implementable in pure Prolog). For CHR, we will be able to answer the above question in a very favorable way: there is no computability penalty, nor is there a penalty in terms of asymptotic complexity. In terms of constant factors, there is a (probably inevitable) penalty, but we will argue that it is very reasonable - about one order of magnitude for time and less than that for space, in current state-of-the-art CHR systems.

\subsection{Overview}

This dissertation consists of 13 chapters, organized in three parts.

\subsubsection{Part I - Background}

The first part introduces the necessary background concepts and the state of the art of optimizing CHR compilation on which the next part will build. Chapter 2 introduces some basic notions of computability and computational complexity theory. Chapter 3 gives an overview of the CHR language: syntax and semantics, program properties, current CHR systems, extensions of CHR, example programs, applications, and related formalisms. Chapter 4 discusses compilation of CHR, the refined operational semantics of $\mathrm{CHR}$ and the related concept of passive oc- 
currences, and a number of compiler optimizations. Finally, Chapter $\mathbf{5}$ demonstrates the applicability of CHR to general-purpose programming by presenting three CHR implementations of classic algorithms.

\subsubsection{Part II - Optimizing Compilation}

In the second part we introduce a number of new compiler optimizations. Chapter 6 introduces a number of guard reasoning techniques that are able to avoid redundant rule guard evaluations and subsumed constraint occurrences by reasoning about the specifics of the execution mechanism that is used (the refined operational semantics). Chapter $\mathbf{7}$ discusses several advanced indexing techniques that improve the performance of rule matching. Chapter 8 introduces two techniques - in-place updates and suspension reuse - to improve the memory usage of CHR systems. Finally, Chapter 9 investigates the join ordering problem for CHR; this work complements Chapter 7 since it is concerned with an orthogonal aspect of rule matching.

\subsubsection{Part III - Computability and Complexity}

The final part deals with the computability and complexity properties of CHR. Chapter 10 introduces a new model of computation, the CHR machine, and shows that it is Turing-complete. Also, some Turing-complete subsets of CHR are identified. Chapter 11 contains the main result of this dissertation: a complexity meta-theorem that can be used to show the complexity-wise completeness of CHR in current CHR systems. It also attempts to extend the results to other declarative languages and considers the constant factors by experimentally evaluating current CHR systems. Chapter 12 generalizes the notion of CHR machines in several ways. Finally, Chapter 13 concludes the text by summarizing the contributions and discussing possibilities for future work.

\subsubsection{Chapter dependencies}

Clearly, parts III and II depend on Part I for the necessary background. Part III depends on Part II in the sense that the optimizations introduced in Part II are needed in order to prove the core complexity results of Part III.

Figure 1.1 shows in more detail how the chapters of this dissertation depend on one another. Readers who wish to skip some chapters or read chapters out of order can consult this dependency graph. For example, if a reader is interested only in the main results of Chapter 11, one way to reach this chapter is by first reading Chapters 3, 4, 7, 8, and 9, then Chapters 2 and 10. 


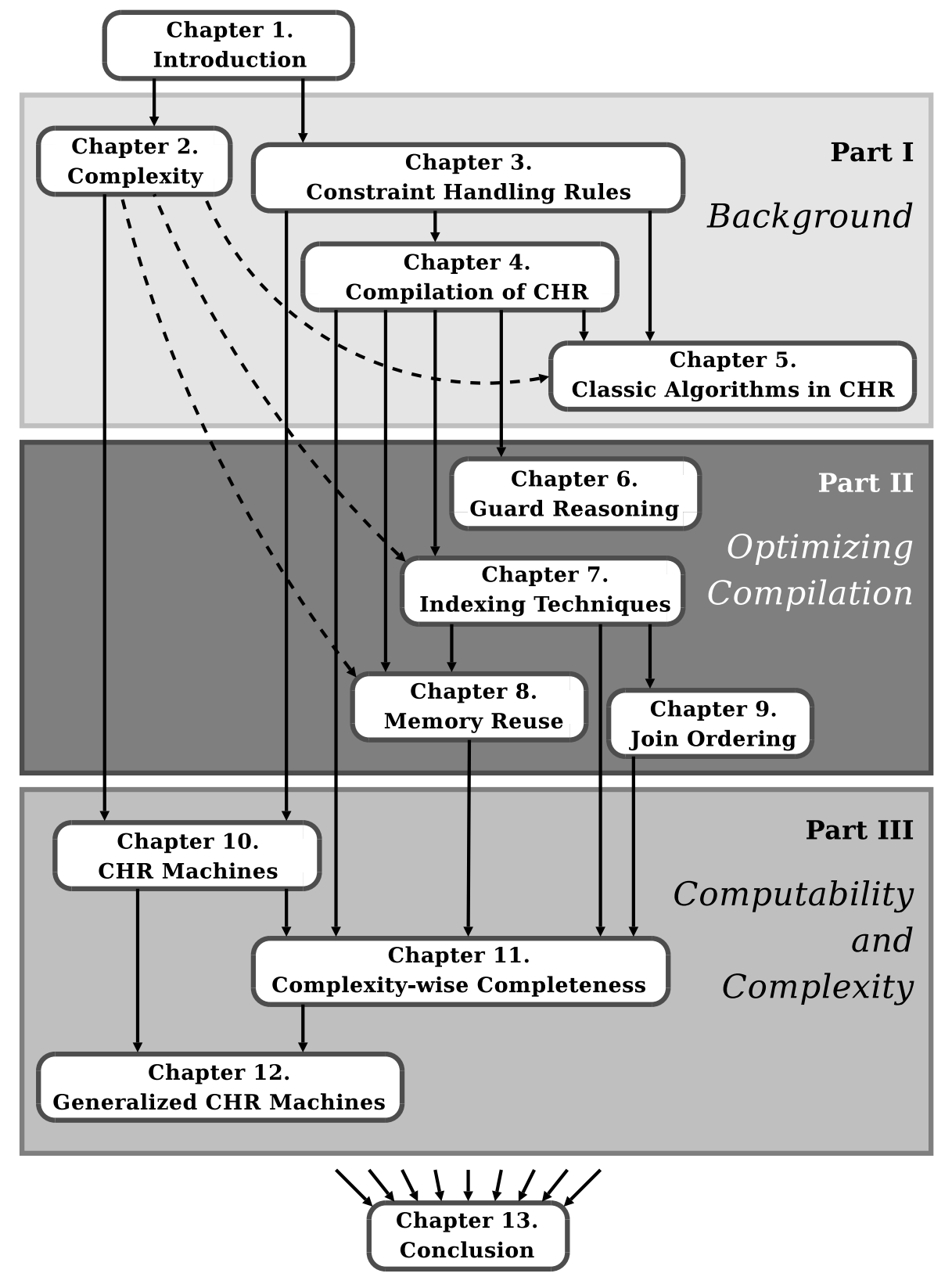

Figure 1.1: Chapter dependencies 


\subsection{Bibliographic notes}

Most of the material presented in this dissertation has been published before and is joint work with other authors, namely Tom Schrijvers, Bart Demoen, Leslie De Koninck, Peter Van Weert, and Thom Frühwirth. In particular:

Chapter 2 is partially based on (Sneyers, Schrijvers, and Demoen 2008a).

Chapters 3 and 4 are partially based on (Sneyers, Van Weert, Schrijvers, and De Koninck 2008).

Section 5.2 is based on (Sneyers, Schrijvers, and Demoen 2006a).

Chapter 6 is based on (Sneyers, Schrijvers, and Demoen 2008b), which was itself based on (Sneyers, Schrijvers, and Demoen 2005c, 2005d).

Chapter 8 is based on (Sneyers, Schrijvers, and Demoen 2006b).

Chapter 9 is based on (De Koninck and Sneyers 2007). The material of this chapter is also presented in the Ph.D. thesis of Leslie De Koninck.

Chapters 10 and 11 are based on (Sneyers, Schrijvers, and Demoen 2008a), which was in turn inspired by (Sneyers, Schrijvers, and Demoen 2005a).

Section 10.3 is based on (Sneyers 2008).

Chapter 12 is based on (Sneyers and Frühwirth 2008).

Some of the material in this dissertation has not been published (yet) at the time of writing. An example is the implementation in CHR of Hopcroft's algorithm for minimizing states of finite automata, which is discussed in Section 5.3.

Finally, some of our CHR-related work has been published but is not included in this dissertation. In particular, we have introduced two extensions of CHR, both adding language constructs to the rule heads. The first extension is the operational notion of negation-as-absence (Van Weert, Sneyers, Schrijvers, and Demoen 2006); the second extension supports (possibly nested) aggregate expressions (Sneyers, Van Weert, and Schrijvers 2007), which was implemented by means of sourceto-source transformation (Van Weert, Sneyers, and Demoen 2008). This work is not discussed in depth in this dissertation. It is only mentioned briefly in sections 3.4.2 and 5.4. In Chapter 6 we have omitted most of the material described in (Sneyers, Schrijvers, and Demoen 2005b) — in particular, the formulation of guard and continuation optimizations in the framework of the call-based refined operational semantics $\omega_{o}$ for occurrence representations of CHR programs - and opted instead for our slightly weaker but conceptually simpler and more general original rule-level approach, as in (Sneyers, Schrijvers, and Demoen 2008b). 


\subsection{Notational conventions}

Most of the symbols used in the text are explained when they are first used. The list of symbols on page 253 gives an overview of every symbol and the page on which it is explained. The following notation is used throughout the text:

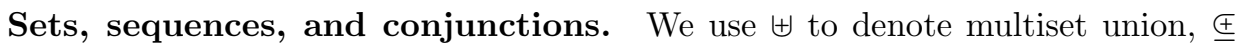
for multiset subset, and + to denote sequence concatenation. We sometimes use Prolog list syntax for sequences: $[h \mid T]$ denotes the list with head element $h$ and tail $T$ which is a list, possibly the empty list. We use $\square$ or [] to denote the empty list. The length of a sequence $X$ is denoted by $\# X$. We will often silently convert between sequences, multisets, and logical conjunctions. Therefore we will usually not assume that logical conjunction is idempotent and commutative, but it is always associative. We use both " $\wedge$ " and comma (",") as a conjunction connective. Given a set $A=\left\{a_{1}, \ldots, a_{n}\right\}$, we use $\bigwedge A$ to denote $a_{1} \wedge \ldots \wedge a_{n}$. As in Prolog, we write the logical truth $T$ as true and logical falsity $\perp$ as fail. The empty sequence corresponds to the 'empty conjunction' true. We use $[l . . u]$ to denote the integer interval between $l$ and $u$ (inclusive).

Variables and quantification. We use vars $(X)$ to denote the set of variables occurring in a syntactic object $X$, We use $\exists_{\left\{v_{1}, \ldots, v_{n}\right\}}$ as a shorthand for $\exists v_{1} \cdots \exists v_{n}$, and $\bar{\exists}_{V} F$ to denote $\exists_{\text {vars }(F) \backslash V} F$, that is, the existential quantification over all variables that are not in the set $V$. We say the variable $v$ is fixed by $B$ (or $B$ fixes $v)$ if $\bar{\exists}_{\{v\}} B \wedge \bar{\exists}_{\{\rho(v)\}} \rho(B) \rightarrow v=\rho(v)$, for an arbitrary renaming $\rho$.

Layout conventions. The symbol "시" is used to indicate remarks for advanced readers. These passages may refer to notions that are only introduced in later chapters, or even not at all. They can always be safely skipped.

(1) Then again, you might miss some juicy details if you do skip these remarks.

All source code fragments are in actual syntax and they are typeset in a typewriter font. When discussing a CHR program, we will often give a name to the program; these names are typeset like this: PROGRAM-NAME.

In listings of CHR programs we use a functional syntax to abbreviate arithmetical expressions (in Prolog): for example, we would write "c $(\mathrm{N}+1)$ " instead of the usual but somewhat more elaborate expression "N1 is $N+1, c(N 1)$ ". 


\section{Part I}

\section{Background}





\title{
Chapter 2
}

\section{Complexity}

The proverbial German phenomenon of the verb-at-the-end about which droll tales of absentminded professors who would begin a sentence, ramble on for an entire lecture, and then finish up by rattling off a string of verbs by which their audience, for whom the stack had long since lost its coherence, would be totally nonplussed, are told, is an excellent example of linguistic recursion.

- Douglas Hofstadter (1945-)

\begin{abstract}
"Instruction tables will have to be made up by mathematicians with computing experience and perhaps a certain puzzle-solving ability. There need be no real danger of it ever becoming a drudge, for any processes that are quite mechanical may be turned over to the machine itself."
\end{abstract}

- Alan Turing (1912-1954)

The theory of computation is one of the most fundamental branches of computer science. In a nutshell, it is the study of whether or not certain problems can be algorithmically solved, both in principle and in practice. The field of computability theory deals with the question of which problems can be solved algorithmically in principle, that is, if there are no time and space constraints. One of the most important results in computability theory is the undecidability of the halting problem (Turing 1936). In the field of (computational) complexity theory, the question is whether a problem can be algorithmically solved in practice. Although there may be an algorithm that solves the problem in principle, it could very well take such a huge amount of time or space - say, it takes longer than the age of the universe to complete the computation, or more bits than the number of particles in the universe - that it is essentially useless in practice. Complexity theory is mainly concerned with the scalability of algorithms, that is, the correlation between the size of the problem instance and the amount of computational resources needed by the algorithm. The most famous open problem in complexity 
theory is the question of whether the complexity classes $\mathbf{P}$ and $\mathbf{N P}$ coincide or not (see Section 2.3.6).

Both in computability theory and in computational complexity theory, idealized formal models of computation are constructed in order to define the notions of "algorithm" and "computational resource" in a precise way. In Section 2.1 we discuss four well-known models of computation: the Turing machine, the RAM (random access memory) machine, the Minsky machine, and the RASP (random access stored program) machine. (In Chapter 10 we will introduce a fifth model of computation, called the CHR machine.) Section 2.2 briefly discusses some elementary notions of computability theory; in particular, the concept of Turingcompleteness and the Church-Turing thesis. Finally, in Section 2.3, we define the notions of time and space complexity in the different models of computation.

\subsection{Models of computation}

Different models of computation have been proposed for different purposes. The well-known Turing machine (Section 2.1.1) is conceptually very simple, which makes it very suitable for theoretical investigation. It also has a non-deterministic variant (Section 2.1.2). An even simpler model of computation is the Minsky machine (Section 2.1.4). The Random Access Memory machine (Section 2.1.3) is somewhat more complicated, but it more closely models realistic computers. The RASP machine (Section 2.1.5) is the most realistic: like real computers, it is based on the von Neumann architecture - memory stores both data and program.

\subsubsection{Turing machines}

The Turing machine, originally introduced by Alan Turing (1936), is the prototypical computational model which is still widely used in computability and complexity theory. We use the single-tape definition of Hopcroft et al. (2001):

Definition 2.1 (Turing machine) $A$ (deterministic) Turing machine is a 6tuple $M=\left\langle Q, \Sigma, q_{0}, b, F, \delta\right\rangle$ where

- $Q$ is a finite set of states;

- $\Sigma$ is a finite set of symbols, the tape alphabet;

- $q_{0} \in Q$ is the initial state;

- $b \in \Sigma$ is the blank symbol;

- $F \subseteq Q$ is the set of accepting final states;

- $\delta: Q \times \Sigma \mapsto Q \times \Sigma \times\{$ left,right $\}$ is a partial function, called the transition function; "left" and "right" represent the direction of the tape head move. 
In the literature definitions sometimes differ subtly, usually without an impact on the computational power or time complexity. For example the set $\{$ left, right is often extended to allow the machine to stay on the same tape cell.

A Turing machine $M$ operates on an infinite tape of cells. Each cell contains a symbol of the tape alphabet $\Sigma$. The tape is assumed to be arbitrarily extendible to the left and the right. A head is positioned on a particular cell of the tape, can read and write a symbol in that cell and can move left and right.

Operation starts in the initial state $q_{0}$ on a tape which contains a finite string of symbols (called the input), and the head is positioned on the left-most input symbol. Execution proceeds by considering the current state $q$ and the symbol $s$ that is under the head. Then:

- Either $(q, s)$ is a member of the domain of $\delta$ and $\delta(q, s)=\left(q^{\prime}, s^{\prime}, X\right)$. The effect then is that the current state of $M$ changes to $q^{\prime}$, the head overwrites the value $s$ in the cell under it with $s^{\prime}$ and next the head either moves to the left or the right depending on whether $X=$ left or $X=$ right.

- Or $(q, s)$ is not part of the domain of $\delta$. Execution stops. If $q \in F$, the input is accepted, otherwise it is rejected.

We represent an execution state of a Turing machine $M=\left\langle Q, \Sigma, q_{0}, b, F, \delta\right\rangle$ as a 4 -tuple $\sigma^{\mathrm{TM}}=\langle q, P, s, N\rangle$, representing the current state $q \in Q$, the current symbol $s \in \Sigma$, the previous symbols $P$ and the next symbols $N$ which are sequences of symbols. We use $\sigma_{0}^{\mathrm{TM}}, \sigma_{1}^{\mathrm{TM}}, \ldots$ to denote Turing machine execution states, and $\Sigma^{\mathrm{TM}}(M)$ to denote the set of all execution states of $M$. Given an input $I=[f \mid N]$ (a sequence of symbols), the initial execution state is $\sigma_{0}^{\mathrm{TM}}=\left\langle q_{0},[], f, N\right\rangle$. The transition rules are:

$$
\begin{aligned}
& \langle q,[p \mid P], s, N\rangle \quad \rightarrow \quad\left\langle q^{\prime}, P, p,\left[s^{\prime} \mid N\right]\right\rangle \\
& \langle q, P, s,[n \mid N]\rangle \rightarrow\left\langle q^{\prime},\left[s^{\prime} \mid P\right], n, N\right\rangle \\
& \langle q,[], s, N\rangle \quad \rightarrow \quad\left\langle q^{\prime},[], b,\left[s^{\prime} \mid N\right]\right\rangle \\
& \langle q, P, s,[]\rangle \rightarrow\left\langle q^{\prime},\left[s^{\prime} \mid P\right], b,[]\right\rangle
\end{aligned}
$$

$$
\begin{array}{ll}
\text { if } & \delta(q, s)=\left(q^{\prime}, s^{\prime}, \text { left }\right) \\
\text { if } & \delta(q, s)=\left(q^{\prime}, s^{\prime}, \text { right }\right) \\
\text { if } & \delta(q, s)=\left(q^{\prime}, s^{\prime}, \text { left }\right) \\
\text { if } & \delta(q, s)=\left(q^{\prime}, s^{\prime}, \text { right }\right)
\end{array}
$$

The machine operates by exhaustively applying the transition rules on the initial execution state. Note that the size of execution states is unbounded but finite. In execution state $\sigma_{0}^{\mathrm{TM}}=\langle q, P, s, N\rangle$, the contents of the tape is the sequence $\operatorname{tape}\left(\sigma_{0}^{\mathrm{TM}}\right)=\operatorname{reverse}(P)+[s]+N$, where reverse reverses a sequence (i.e. reverse $\left.\left(\left[a_{1}, a_{2}, \ldots, a_{n-1}, a_{n}\right]\right)=\left[a_{n}, a_{n-1}, \ldots, a_{2}, a_{1}\right]\right)$. If the final execution state is of the form $\left\langle q_{f}, P, s, N\right\rangle$, the Turing machine has accepted the input $I$ if $q_{f} \in F$. If $q_{f} \in Q \backslash F$, the input is rejected. When the input is accepted, the output of the Turing machine is the tape contents in the final execution state; when the input is rejected, the output is undefined. Given a Turing machine $M$ and input $I$, we denote the corresponding derivation by $\operatorname{deriv}_{M}(I)$. If the machine terminates on input $I$, we denote the output by $M(I)=\operatorname{tape}\left(\sigma_{f}^{\mathrm{TM}}\right)$, where $\sigma_{f}^{\mathrm{TM}}$ is the last state in $\operatorname{deriv}_{M}(I)$. 


\subsubsection{Non-deterministic Turing machines}

In deterministic Turing machines, there is exactly one next step (or none, for the configurations in which the machine terminates) for every combination of a state and an input symbol. Non-deterministic Turing machines do not have this restriction: there can be different actions for the same state and input symbol.

Definition 2.2 (non-deterministic TM) A non-deterministic Turing machine is a 6 -tuple $M=\left\langle Q, \Sigma, q_{0}, b, F, \delta\right\rangle$ which is defined as in Definition 2.1, except for $\delta$, which is an arbitrary relation (not necessarily a function):

$$
\delta \subseteq(Q \times \Sigma) \times(Q \times \Sigma \times\{\text { left, right }\})
$$

A non-deterministic Turing machine works just like a deterministic one, except that whenever there are $n>1$ possible actions to be taken, $n$ duplicates of the machine are made, and every choice is executed in parallel. As soon as one of the execution branches halts in an accepting final states, the entire non-deterministic Turing machine stops and accepts the input (the output is the tape content of the accepting branch). When all execution branches have halted in a rejected final state, the entire non-deterministic Turing machine stops and rejects the input. Otherwise, the machine does not terminate.

(1) Another way of looking at non-deterministic Turing machines is as follows. Whenever there is a choice to be made, the machine takes the "right" choice, that is, a choice that leads to an accepting final state. If there is no such series of choices, arbitrary choices are made. In this sense, a non-deterministic Turing machine, when faced with a choice, is the luckiest possible guesser.

\subsubsection{RAM machines}

The Random Access Memory (RAM) machine (Aho, Hopcroft, and Ullman 1975) closely models the basic features of traditional sequential computers. In the literature many variations of the RAM have been considered. We investigate two different RAM machines. The first is a RAM machine with simple Peano arithmetic operations and the second has the standard arithmetic operations as they are implemented on today's computers.

\section{Common architecture}

A RAM machine consists of three components: the central processing unit (CPU), the program, and the random-access memory (RAM). The memory consists of an infinite number of cells, or registers, which are labeled with a natural number which is called its address. If a register is initialized, it contains a value, which is 


\begin{tabular}{lll|l}
\hline Instruction & & & Effect \\
\hline inc & $A$ & & $\llbracket A \rrbracket \leftarrow \llbracket A \rrbracket+1$ \\
dec & $A$ & & $\llbracket A \rrbracket \leftarrow \llbracket A \rrbracket-1$ \\
clr & $A$ & & $\llbracket A \rrbracket \leftarrow 0$ \\
jmp & $L$ & & $P C \leftarrow L$ \\
cjmp & $A$ & $L$ & $P C \leftarrow L$ if $\llbracket A \rrbracket=0$ \\
halt & & & Halt execution of the RAM machine \\
\hline init & $A$ & & Initialize the register at address $\llbracket A \rrbracket$ to zero \\
cnst & $B$ & $A$ & $\llbracket A \rrbracket \leftarrow B$ \\
add & $A_{2}$ & $A_{1}$ & $\llbracket A_{1} \rrbracket \leftarrow \llbracket A_{1} \rrbracket+\llbracket A_{2} \rrbracket$ \\
sub & $A_{2}$ & $A_{1}$ & $\llbracket A_{1} \rrbracket \leftarrow \llbracket A_{1} \rrbracket-\llbracket A_{2} \rrbracket$ \\
mul & $A_{2}$ & $A_{1}$ & $\llbracket A_{1} \rrbracket \leftarrow \llbracket A_{1} \rrbracket * \llbracket A_{2} \rrbracket$ \\
div & $A_{2}$ & $A_{1}$ & $\llbracket A_{1} \rrbracket \leftarrow \llbracket A_{1} \rrbracket / \llbracket A_{2} \rrbracket$ if $\llbracket A_{2} \rrbracket \neq 0$ \\
mov & $A_{2}$ & $A_{1}$ & $\llbracket A_{1} \rrbracket \leftarrow \llbracket A_{2} \rrbracket$ \\
imv & $A_{2}$ & $A_{1}$ & $\llbracket A_{1} \rrbracket \leftarrow \llbracket \llbracket A_{2} \rrbracket \rrbracket$ \\
mvi & $A_{2}$ & $A_{1}$ & $\llbracket \llbracket A_{1} \rrbracket \rrbracket \leftarrow \llbracket A_{2} \rrbracket$ \\
\hline
\end{tabular}

Table 2.1: Instruction set of the RAM machine

an integer number. We use $A, A_{1}, A_{2}, \ldots$ to represent the memory addresses and $\llbracket A \rrbracket$ to denote the value of the register at address $A$.

The program consists of a sequence of instructions. The program instructions are labeled with successive natural numbers. We use $L, L_{1}, \ldots$ to denote program instruction labels. The CPU follows a fetch-and-execute cycle. It has a program counter $P C$ that is initialized to the first program instruction label. This program counter contains the label of the next program instruction to be executed. The $\mathrm{CPU}$ fetches the instruction and performs the corresponding operations. ${ }^{1}$ This involves setting the program counter to the next instruction, by default the successor of the current address. Table 2.1 lists the instructions supported by the standard RAM machine. The Peano-arithmetic RAM machine uses a subset of these instructions.

Definition 2.3 (Peano-arithmetic RAM machine) A Peano-arithmetic RAM machine consists of a program and a working memory as described above. The program instructions are inc, dec, clr, jmp, cjmp, and halt (see Table 2.1).

This corresponds to the definition used by Savage (1998). Indirect addressing is not supported, so all registers that are used in a program are supposed to be initialized in advance. All copying, addition and subtraction has to be done by repeated use of the inc and dec instructions. This makes the Peano-arithmetic

\footnotetext{
${ }^{1}$ If an illegal instruction is encountered, the machine halts. Examples of illegal instructions are division by zero, jump to a non-existent label, instructions referring to registers with a negative address, etc.
} 
RAM less practical, as actual computers do provide instructions for addition and subtraction.

(1) Pure CHR without built-in constraints does not provide any arithmetic functionality either. Hence, arithmetic must be encoded, for example using Peano arithmetic.

Definition 2.4 (standard RAM machine) A standard-arithmetic RAM machine consists of a program and a working memory as described above. The program instruction set is given in Table 2.1.

This instruction set is similar to the definition of Aho et al. (1975), and resembles more closely actual computers. The inc, dec, and clr instructions are redundant: they can be implemented using add, sub, and cnst. Without loss of generality, we assume the instructions that refer to two (add, sub, mul, div, mov) or three (imv, mvi) memory cells refer to two or three different cells. For example, the instruction "add $a$ a" should be rewritten to "mov $a t$; add $t a$ " where $t$ is some temporary register.

By convention, particular memory cells are initialized in advance and contain the input, while other (or the same) memory cells are meant to contain the output. Without loss of generality we assume that the program initializes all additional registers (using the init instruction) before they are used.

(1) A program that does not initialize registers before it uses them can be rewritten in the following way. We may assume all registers that are used directly in operands to be initialized in advance (these can be easily found by inspecting the program). We let the program instructions that use indirect addressing (imv and mvi) be preceded by an init instruction. For example, the instruction "mvi $a b$ " should be rewritten to "init $b$; mvi $a b$ ". This transformation at most doubles the program size and the number of instructions executed.

\subsubsection{Minsky machines}

An even simpler version of the Peano-arithmetic RAM machine was proposed by Minsky (1967). Minsky machines have only two registers, that can hold natural numbers. There are only two instructions: $\operatorname{succ}\left(r_{i}\right)$, which increases the value of $r_{i}$ by one, and decjump $\left(r_{i}, p_{j}\right)$, which decreases the value of $r_{i}$ by one if it is nonzero, and otherwise jumps to program line $p_{j}$. A program consists of a numbered sequence of instructions and unless there is a jump, the program continues with the next instruction in the sequence. Some program lines may be empty; in that case the machine halts (in some formulations a third instruction halt is used to represent these empty lines). Without loss of generality we can assume that both registers are initialized to zero.

- Although the Minsky machine is very simple, it is still a Turing-complete model of computation (Minsky 1967). 


\subsubsection{RASP machines}

The RASP (random access stored program) machine (Hartmanis 1971) is essentially a RAM machine which can access and change its own program instructions much like real computers which follow the von Neumann architecture: instructions and data are stored in the same memory space, hence the term stored program.

Using some kind of encoding, program instructions can be represented as a number. How exactly the instructions are encoded is not important for our purposes. The program counter now points to a register address - in fact, the program counter itself could also be stored in a register (in that case, the jmp instruction can be alternatively implemented by overwriting that register). The fetch-and-execute cycle consists of decoding the instruction to which the program counter points and executing it.

The main difference between a RASP machine and a RAM machine is that RASP machines allow self-modifying programs, whereas in a RAM machine, the program is immutable. As a consequence, the indirect addressing instructions (imv and mvi) are not needed: instead, the program can use a mov instruction of which it modifies the operands as needed.

\subsection{Computability}

Computability theory studies the boundary between the problems that can be solved algorithmically, and those that cannot. An algorithm that solves a problem (also called an effective method or effective procedure) is a method consisting of steps that may be described as mechanical operations; a method which, if followed rigorously, has the following properties:

- the method always terminates after a finite number of steps;

- the method always gives the correct answer and never a wrong answer;

- the method works for all instances of the problem.

Note that this is not a mathematically precise definition - the notion of effective procedure is somewhat vague and intuitive, in particular because it is not specified what kind of operations "may be described as mechanical operations". The models of computation of the previous section were formulated in an attempt to formalize this notion of an algorithm.

\subsubsection{Church-Turing thesis}

The Church-Turing thesis can be formulated as follows: "A problem can be algorithmically solved if and only if there is a Turing machine that solves it." In other words, the notion of computability by a Turing machine and the intuitive 
notion of effective computability are in fact the same. This is not a mathematical statement that can be proven correct formally because it is a statement about the informal notion of an algorithm.

It is supported by the equivalence of Turing machines and $\lambda$-calculus, a formal system introduced by Alonzo Church and Stephen Kleene in the 1930s. Both formalisms were introduced independently to capture what is computable. They are very different formalisms, originating from a different approach to computability: the "mechanical" approach in the case of Turing machines and the "mathematical" or "functional" approach in the case of $\lambda$-calculus. Still, both formalisms were shown to be equivalent - which indicates that either formalism really corresponds to the intuitive notion of "effective computability".

According to the Church-Turing thesis, all 'reasonable' models of computation are equivalent to the Turing machine in the sense that they can all solve the same problems. Today, about 70 years after its first formulation, the Church-Turing thesis is widely believed to hold. Indeed, for all reasonably strong models of computation that have been proposed in the literature, it has been proved that they are (computationally) equivalent to the Turing machine. Clearly, models of computation can also be defined that are weaker than Turing machines - for example, finite automata.

\subsubsection{Turing-completeness}

A model of computation is called Turing-complete if it has (at least) the same computational power as Turing machines, that is, every Turing Machine can be simulated in the model. A model of computation is called Turing-equivalent if it has precisely the same computational power as Turing machines, i.e. it is Turingcomplete and every program of the model can also be simulated on a Turing machine. All 'reasonable' Turing-complete models of computation are also Turingequivalent ${ }^{2}$, a fact that adds support to the Church-Turing thesis.

A universal Turing machine is a Turing machine program that can "run" any arbitrary Turing machine: if the tape is initialized with some encoding of a Turing machine program followed by an input tape for that program, the final output on the tape is exactly the same as the output the encoded program would give. The existence of such universal machines is arguably one of the most important results in the whole of computer science, and it is due to Turing (1936). The von Neumann architecture and concepts like program-as-data, subroutines, compilers, operating systems, ... were directly influenced by the existence of universal machines.

\footnotetext{
${ }^{2}$ Some contrived models of computation that are not intended to model some physically realizable computational device are not 'reasonable'. For example, so-called oracle machines are more powerful than plain Turing machines if the oracle is sufficiently powerful, for instance capable of solving the halting problem in a single step. Another example is the so-called Zeno machine, in which each computational step takes half the time of the previous step - such a machine can perform a countably infinite number of steps in finite time.
} 
"[...] I would like to allow myself to write you about a mathematical problem, of which your opinion would very much interest me: One can obviously easily construct a Turing machine, which for every formula $F$ in first order predicate logic and every natural number n, allows one to decide if there is a proof of $F$ of length $n$ (length = number of symbols). Let $\psi(F, n)$ be the number of steps the machine requires for this and let $\phi(n)=\max _{F} \psi(F, n)$. The question is how fast $\phi(n)$ grows for an optimal machine. One can show that $\phi(n) \geq k \cdot n$. If there really were a machine with $\phi(n) \sim k \cdot n$ (or even $\sim k \cdot n^{2}$ ), this would have consequences of the greatest importance. Namely, it would obviously mean that in spite of the undecidability of the Entscheidungsproblem, the mental work of a mathematician concerning Yes-or-No questions could be completely replaced by a machine. After all, one would simply have to choose the natural number $n$ so large that when the machine does not deliver a result, it makes no sense to think more about the problem. Now it seems to me, however, to be completely within the realm of possibility that $\phi(n)$ grows that slowly. [...]"

Figure 2.1: Letter from Kurt Gödel to John von Neumann, 20 March 1956

\subsection{Computational complexity theory}

Complexity theory investigates the amount of computational resources needed to execute an algorithm. The two most important computational resources are time (execution time - number of steps) and space (size of the required memory number of tape cells or registers).

One of the seminal papers that founded and shaped the field of computational complexity theory was (Hartmanis and Stearns 1965). Relatively recently, in 1988, a letter surfaced that indicates that the core idea and importance of complexity theory was already recognized by Kurt Gödel as early as 1956. A fragment of this letter ${ }^{3}$ is shown in Figure 2.1.

Essentially, Gödel argues in the letter that he considers it to be possible that, using current terminology, QBF (quantified Boolean formula) can be solved in linear or quadratic time. He clearly realizes the tremendous consequences of this possibility, which would imply that $\mathbf{P}=\mathbf{P S P A C E}$ and so also $\mathbf{P}=\mathbf{N P}$. Even today, Gödel's question remains an open problem, although the majority of complexity theorists now put their bets on $\mathbf{P} \subsetneq$ PSPACE.

\footnotetext{
${ }^{3}$ The full letter can be found at http://www. contrib.andrew. cmu. edu/ hardt/godel.html.
} 


\subsubsection{Time complexity}

The worst-case time complexity of an algorithm or program is defined as a function of the size of the input. For a given input size, the worst-case time complexity is the maximal number of execution steps needed for executing the program on arbitrary input of that size.

It is important to make explicit, for a given problem, how the size of a problem instantiation (the input size) is to be measured. For example, if the problem is testing whether or not a number is a prime, one could count the number itself as the size of the input or, alternatively, the number of bits (or decimal digits) needed to represent the number. Depending on which input size measure is used, the time complexity of the AKS primality test (Agrawal, Kayal, and Saxena 2004) is polylogarithmic or polynomial, respectively.

In some cases the related notions of average-case and best-case complexity may also be useful. Usually, we are interested in performance guarantees, for which the worst-case is most important. From now on, when we say "complexity", we implicitly mean the worst-case complexity.

For deterministic Turing machines, the length \# $\operatorname{deriv}_{M}$ of a derivation $\operatorname{deriv}_{M}$ is simply the number of steps in that derivation. Recall that for non-deterministic Turing machines, we consider the shortest possible derivation that leads to an accepting final state; if there are no accepting paths, the derivation length is defined to be zero.

Definition 2.5 (time complexity of Turing machines) The time complexity of a Turing machine $M=\left\langle Q, \Sigma, q_{0}, b, F, \delta\right\rangle$ is the maximal derivation length for inputs of a given size:

$$
\operatorname{TMTime}_{M}(n)=\max \left\{\# \operatorname{deriv}_{M}(I) \mid I \in \Sigma^{*} \text { and }|I|=n\right\}
$$

Definition 2.6 (time complexity of RAM machines) The time complexity $\operatorname{RAMTime}_{P}(n)$ of a RAM machine with program $P$ is the maximal time needed for an execution starting with some input of size $n$. The time needed for a program execution is the sum of the times needed for every instruction that is executed. All instructions take constant time except for the arithmetic instructions add, sub, mul, and div, which take $O(\log |x|)$ time where $x$ is the largest (in absolute value) of the numbers involved in the arithmetic operation.

\subsubsection{Space complexity}

The space complexity of an algorithm is the number of tape cells (or bits) it needs, in the worst case, during its execution. As time complexity, it is defined as a function of the input size. 
Definition 2.7 (space complexity of Turing machines) The space complexity of a Turing machine $M$ is the maximal tape size used in derivations for inputs of a given size:

$$
\operatorname{TMSPACE}_{M}(n)=\max \left\{\# \operatorname{tape}(\sigma) \mid \sigma \in \operatorname{deriv}_{M}(I) \text { and } I \in \Sigma^{*} \text { and }|I|=n\right\}
$$

Definition 2.8 (space complexity of RAM machines) The space complexity $\operatorname{RAMSPACE}_{P}(n)$ of a RAM machine with program $P$ is the maximum, over every execution starting with an input of size $n$, of the sum over all registers in the range $0, \ldots$, maxaddr of the number of bits needed to represent the maximal value that it held. Unused registers within that range are charged one bit for the implicit value 0 stored in them.

In practice, we often assume the registers to use at most a fixed number of bits. In that case, every instruction takes constant time and the space complexity is just maxaddr, the maximum register address reached in a computation.

(1) The above Definition 2.8 corresponds to the one proposed by Savitch (1978). In the literature, other definitions of space complexity are often used, that do not count the unused registers (see also (van Emde Boas 1990), page 28, for a discussion of different space complexity measures). Consider a program that uses the registers with addresses $2^{1}, 2^{2}, 2^{3}, \ldots, 2^{n}$ if the input size is $n$. If unused registers are not taken into account, the space complexity of this program is only linear; according to our definition it uses exponential space. When unused registers are not counted, programs never use more space than time (and this seems to be a desirable property). However, in our opinion the definition of (Savitch 1978) is more realistic.

In the following, we will assume RAM programs to use at least as much time as space. This is a nontrivial assumption given our definition of space complexity, but unless a program uses the registers in an unrealistically sparse way, it is not a very restrictive assumption. After all, every program can be rewritten to use memory in a dense way with only logarithmic time overhead, e.g. by explicitly storing address-value pairs using a balanced search tree.

\subsubsection{Asymptotic complexity and big $\mathrm{O}$ notation}

We are usually not interested in the exact time or space complexity of an algorithm, but only in its scalability. The practical reason is that real computers tend to become faster and faster and the amount of physical memory also tends to increase. Hence, if I have an algorithm which is a constant factor $k$ slower than your algorithm, we can still handle the same range of input sizes - I just have to buy a more expensive computer that is $k$ times as fast as yours, or wait until such computers become available. However, if my algorithm takes $2^{n}$ steps for input of size $n$ and your algorithm takes $n^{2}$ steps, then there will always be inputs that you 


\begin{tabular}{c|c|l}
\hline Complexity & Name & Example problem (algorithm) \\
\hline$O(1)$ & constant & Accessing an arbitrary element of an array \\
$O(\log n)$ & logarithmic & Searching in a sorted list of length $n$ \\
$O(n)$ & linear & Searching in an unsorted list of length $n$ \\
$O(n \alpha(n))$ & inverse Ackermann & Performing $n$ union-find operations \\
$O(n \log n)$ & quasilinear & Sorting a list of $n$ elements (heapsort) \\
$O\left(n^{2}\right)$ & quadratic & Sorting a list of $n$ elements (insertion sort) \\
$O\left(n^{3}\right)$ & cubic & Finding a shortest path in a weighted graph \\
& & (where edge weights may be negative) with $n$ nodes \\
$O\left(n^{k}\right)$ & polynomial & (Bellman-Ford algorithm) \\
& Testing whether a number of $n$ digits is prime \\
$O\left(k^{n}\right)$ & exponential & (Agrawal-Kayal-Saxena primality test) \\
$O(n !)$ & Tractorial & Traveling salesman problem (dynamic programming) \\
\hline
\end{tabular}

Table 2.2: Common asymptotic complexities

can handle but I cannot (in, say, one human lifetime), no matter how much faster my computer is compared to yours. A more theoretical reason to ignore constant factors is the so-called linear speedup theorem (Hartmanis and Stearns 1965): if a problem can be solved by a Turing machine in time $f(n)$, there is also a Turing machine that solves it in time $c f(n)+n+2$, for any $c>0$.

The notion of asymptotic complexity is used to compare the scalability of algorithms. A function $f(n)$ is asymptotically bounded by another function $g(n)$, which we denote by saying $f(n)$ is $O(g(n))$, if and only if

$$
\exists N, c>0: \forall n>N: f(n) \leq c g(n)
$$

If algorithm $A$ has complexity $f(n)$ and algorithm $B$ has complexity $g(n)$, and $f(n)$ is $O(g(n))$, then we say algorithm $A$ is at least as efficient as algorithm $B$. If both $f(n)$ is $O(g(n))$ and $g(n)$ is $O(f(n))$, we say that $f(n)$ is $\Theta(g(n))$.

Table 2.2 lists some common asymptotic time complexities, ordered from most efficient to least efficient. In this table, $n$ denotes the input size and $k$ denotes some constant. We also use the notation $\tilde{O}(f(n))$ as a shorthand for $O\left(f(n) \log ^{k} f(n)\right)$, for some fixed $k$. In this notation, polylogarithmic factors are ignored. Polylogarithmic factors are often not that important since any function that is $\tilde{O}\left(n^{k}\right)$ is also $O\left(n^{k+\epsilon}\right)$, for arbitrary small $\epsilon>0$.

\subsubsection{Amortized complexity analysis}

The execution of many algorithms, in particular data structure algorithms, consists of a sequence of operations. In most circumstances (the exception being real-time programs) we are only interested in the total time, not in the time per operation. 
Sometimes the time per operation varies a lot, and as a result, the worst-case time per operation would seem rather bad. However, if the worst case only happens demonstrably rarely, the worst-case time for sequences of operations can be much better than the naive bound obtained by multiplying the length of the sequence by the worst-case time per operation.

The amortized complexity of an operation is the average time needed for the operation, over a worst-case sequence of operations. As a simple example, consider a data structure which has two operations: $\operatorname{push}(x)$, which puts some item $x$ at the top of a stack, and popall, which removes all items from the stack while printing them. Clearly, if there are $n$ elements on the stack, the time needed for the popall operation is $O(n)$. Given a sequence of $k$ operations, the stack size may be as large as $k$, so the total time is $O\left(k^{2}\right)$. Using amortized complexity analysis, we can improve this bound. Both operations take only constant amortized time.

This can be seen as follows. We assign a potential, a positive number, to every possible state of the stack data structure. The amortized time for an operation is its actual time plus the net increase in potential caused by it. The actual time for a sequence of operations is then the total amortized time minus the total net increase in potential. We define the potential of a stack simply as the number of elements it contains. At the beginning of a sequence of operations we have an empty stack, so the initial potential is zero. Since the potential cannot be negative, the amortized time complexity is an upper bound for the actual time complexity. The push operation indeed has a constant amortized time complexity: adding one element to the stack takes constant time, and the potential of the stack is increased by one, which is also a constant. The popall operation takes $n$ time units if the stack has size $n$, but the increase in potential it causes is $-n$, so its amortized complexity is also constant. We can conclude that any sequence of push and popall instructions of length $k$ takes only $O(k)$ time.

In Section 5.2.4 we give a more interesting example of amortized complexity analysis when discussing the time complexity of the Fibonacci heap algorithm of Fredman and Tarjan (1987).

\subsubsection{Relation between RAM machines and Turing machines}

It is easy to see that RAM machines are Turing-complete. Both kinds of RAM machines can simulate a $T$-time Turing machine in $O(T)$ time. Also, both kinds of RAM machines are Turing-equivalent. The main difference between the two RAM machines is the time complexity that can be achieved when simulating them on a Turing machine. According to Savage (1998), a T-time Peano-arithmetic RAM using $S$ registers can be simulated on a Turing machine in $O\left(S T \log ^{2} S\right)$ time. The standard RAM is also polynomially related to the Turing machine, although it is more expensive to simulate on a TM. According to Aho et al. (1975), a standard RAM machine with time complexity $T$ can be simulated on a multi-tape TM in 
$O\left((T \log T \log \log T)^{2}\right)$ time. Simulating a multi-tape TM on a single-tape TM squares the time complexity (Hartmanis and Stearns 1965), so we have:

Lemma 2.9 Any standard RAM machine with time complexity $T$ can be simulated on a Turing machine with time complexity $\tilde{O}\left(T^{4}\right)$.

\subsubsection{Complexity classes}

A language over a finite alphabet (a finite set of symbols) is a possibly infinite set of strings (finite sequences of symbols from the alphabet). The set of all strings over an alphabet $\Sigma$ is denoted by $\Sigma^{*}$. For a language $L \subseteq \Sigma^{*}$, a corresponding decision problem can be defined: given a string $x \in \Sigma^{*}$, is $x \in L$ or not?

The complexity class $\mathbf{P}$ is defined as the set of languages which can be decided by a (deterministic) Turing machine in polynomial time. In other words, if $L \in \mathbf{P}$, then there is a Turing machine that always terminates in $O\left(n^{k}\right)$ steps (for some fixed $k$ ), and accepts the input if and only if it is in $L$. Because of RAM machines and Turing machines are polynomially related (cf. Lemma 2.9), this definition can equivalently be formulated in terms of RAM machines.

Similarly, the complexity class NP is defined as the set of languages which can be decided by a non-deterministic Turing machine in polynomial time. Clearly, $\mathbf{P} \subseteq \mathbf{N P}$. One of the most important open problems in computer science is whether $\mathbf{P}=\mathbf{N P}$ or $\mathbf{P} \neq \mathbf{N P}$ (the prevailing assumption is $\mathbf{P} \neq \mathbf{N P}$ ).

The complexity class co-NP is defined as the set of languages whose complement is in NP. The complement $\bar{L}$ of a language $L$ over an alphabet $\Sigma$ is the set of all strings that are not in $L: \bar{L}=\left\{x \in \Sigma^{*} \mid x \notin L\right\}$. Another open problem is whether $\mathbf{N P}=\mathbf{c o}-\mathbf{N P}$ or not. If $\mathbf{N P} \neq \mathbf{c o}-\mathbf{N P}$, then $\mathbf{P} \neq \mathbf{N P}$.

A language $L \in \mathbf{N P}$ is called NP-complete if for every language $K \in \mathbf{N P}$, there is a polynomial-time reduction to $L$, that is, a (deterministic) polynomialtime algorithm which transforms instances $x_{K} \in \Sigma^{*}$ to instances $x_{L} \in \Sigma^{*}$ in such a way that $x_{K} \in K$ if and only if $x_{L} \in L$. This implies that if a polynomialtime algorithm can be found for deciding one NP-complete language, then all languages in NP can be decided in polynomial time, and thus $\mathbf{P}=\mathbf{N P}$. Many natural problems are NP-complete (or have a corresponding decision problem that is). Example are the boolean satisfiability problem (SAT), the traveling salesman problem (TSP), finding Hamiltonian paths, and graph coloring. The corresponding complexity class is called NPC. Analogously, the complexity class co-NPC is defined as the set of languages in co-NP to which all co-NP languages are reducible in polynomial time.

The above complexity classes are defined in terms of time complexity, but it can also be useful to consider space complexity instead. The complexity class PSPACE is defined as the set of languages which can be decided by a (deterministic) Turing machine in polynomial space. It can be shown that NP $\subseteq$ PSPACE, but it is not known whether the inclusion is strict. 


\title{
Chapter 3
}

\section{Constraint Handling Rules}

\author{
"[..] Sir Isaac Newton, renowned inventor of the milled-edge coin \\ and the catflap!" \\ "The what?" said Richard. \\ "That catflap! A device of the utmost cunning, perspicuity and \\ invention. It is a door within a door, you see, a ..." \\ "Yes," said Richard, "there was also the small matter of gravity." \\ "Gravity," said Dirk with a slightly dismissed shrug, "yes, there \\ was that as well, I suppose. Though that, of course, was merely \\ a discovery. It was there to be discovered." ... "You see?" he \\ said dropping his cigarette butt, "They even keep it on at weekends. \\ Someone was bound to notice sooner or later. But the catflap ... ah, \\ there is a very different matter. Invention, pure creative invention. \\ It is a door within a door, you see."
}

- Douglas Adams (1952-2001)

In this chapter we give an introduction to the CHR language, in terms of both theory and practice - that is, the practice of programming in CHR. The next chapter covers the practice of CHR from the perspective of the implementation of CHR compilers.

For other, more gentle or more complete introductions to CHR we refer to any of the following: Frühwirth (1998), Frühwirth and Abdennadher (2003), Schrijvers (2005), Duck (2005), Sneyers et al. (2008), and Frühwirth (2009). Readers that are already familiar with CHR can skip this chapter, except for Section 3.1.5 which introduces some new terminology. All other material in this chapter consists of the usual definitions as in the above literature. 


\subsection{Syntax and semantics of CHR}

In this section we introduce the syntax and semantics of Constraint Handling Rules. We assume the reader to be familiar with some basic notions of contraint logic programming (cf. Section 1.1.2).

\subsubsection{Syntax}

First of all, it should be clear that CHR is not meant to be used as a stand-alone programming language in itself. Instead, it is a language extension that adds user-defined constraints and rules to handle them to some given host language. We always assume that CHR is embedded in a host language $\mathcal{H}$ that provides data types and a number of predefined constraints. These predefined constraints are called host language constraints or built-in constraints. The traditional host language of CHR is Prolog. Its only host language constraint is equality of Herbrand terms (solved using Prolog's built-in unification); its data types are Prolog variables and terms. We denote the host language in which $\mathrm{CHR}$ is embedded between round brackets: i.e. $\operatorname{CHR}(\mathcal{H})$ denotes $\mathrm{CHR}$ embedded in host language $\mathcal{H}$. Most systems are $\mathrm{CHR}($ Prolog) systems, but there are also several implementations of $\mathrm{CHR}($ Java) and $\mathrm{CHR}($ Haskell), and recently a $\mathrm{CHR}(\mathrm{C})$ system was developed. We discuss these CHR systems in Section 11.2.

\section{Host language requirements}

We assume the host language to offer at least one data type that can be used as an identifier, i.e. a data type that allows the following two operations: creation of a new unique value, and equality testing; for instance Prolog variables or integers. We denote the built-in constraint theory by $\mathcal{D}_{\mathcal{H}}$ and we assume that it defines at least the basic constraints true, the empty constraint which is trivially satisfied; fail, a contradictory constraint which is unsatisfiable; and the ask-versions of syntactic equality ("==") and inequality ("\==").

\section{Syntax of CHR}

CHR constraint symbols are drawn from the set of predicate symbols, denoted by a functor/arity pair (the functor is the name of the predicate, the arity is the number of arguments). CHR constraints, also called constraint atoms or constraints for short, are atoms constructed from these symbols and the data types provided by the host language. A query or goal is a conjunction (or multiset, in an abstract setting) of both CHR and host language constraints. We denote the set of goals for a given $\mathrm{CHR}$ program $\mathcal{P}$ and host language $\mathcal{H}$ by the symbol $\mathcal{G}_{\mathcal{P}}^{\mathcal{H}}$.

A CHR program $\mathcal{P}$ consists of a sequence of CHR rules. There are three kinds of rules: (where $k, l, m, n \geq 1$ ) 
- Simplification rules:

$$
\begin{aligned}
& h_{1}, \ldots, h_{n} \Longleftrightarrow g_{1}, \ldots, g_{m} \mid b_{1}, \ldots, b_{k} . \\
& h_{1}, \ldots, h_{n} \Longrightarrow g_{1}, \ldots, g_{m} \mid b_{1}, \ldots, b_{k} . \\
& \text { - Simpagation rules: } \quad h_{1}, \ldots, h_{l} \backslash h_{l+1}, \ldots, h_{n} \Longleftrightarrow g_{1}, \ldots, g_{m} \mid b_{1}, \ldots, b_{k} \text {. }
\end{aligned}
$$

- Propagation rules:

The sequence, or conjunction, $h_{1}, \ldots, h_{n}$ consists of CHR constraints; together they are called the head or head constraints of the rule. A rule with $n$ head constraints is called an $n$-headed rule and when $n>1$, it is a multi-headed rule.

All the head constraints of a simplification rule and the head constraints $h_{l+1}, \ldots, h_{n}$ of a simpagation rule are called removed head constraints. The other head constraints - all heads of a propagation rule and $h_{1}, \ldots, h_{l}$ of a simpagation rule - are called kept head constraints. An occurrence number is associated with every head constraint. Head constraints are numbered per functor/arity pair, starting from 1, from the first rule to the last rule, removed heads before kept heads, from left to right.

The conjunction $b_{1}, \ldots, b_{k}$ consists of CHR constraints and host language constraints; it is called the body of the rule. The part of the rule between the arrow and the body is called the guard. It is a conjunction of host language constraints. The guard " $g_{1}, \ldots, g_{m} \mid$ " is optional; if omitted, it is considered to be "true $\mid$ ".

(1) The guard is allowed to contain variables that do not occur in the head of the rule - these variables are existentially quantified. If these variables get instantiated during evaluation of the guard, this is 'undone' when the body is executed (if the variables are also used in the body), that is, according to the usual definitions of the operational semantics $\left(\omega_{t}\right.$ and $\left.\omega_{r}\right)$. However, many actual CHR implementations (cf. Chapter 4) do not 'undo' the execution of the guard, and as a result, for instance, the following rule does get the expected behavior in most CHR systems:

list(L) \choose_one $\Leftrightarrow$ member $(\mathrm{X}, \mathrm{L})$ | chose $(\mathrm{X})$.

In general, in rules where the guard and the body share variables that do not occur in the head, we can always transform a rule of the form $H \Longleftrightarrow G \mid B$ to a rule of the form $H \Longleftrightarrow G \mid G, !, B$ in order to obtain the expected behavior.

A rule is optionally preceded by name @ where name is a term. No two rules may have the same name. If a rule does not have a name, it gets a unique name implicitly.

For simplicity, both simplification and propagation rules are often treated as special cases of simpagation rules. The following notation is used:

$$
H^{k} \backslash H^{r} \Longleftrightarrow G \mid B
$$

If $H^{k}$ is empty, then the rule is a simplification rule. If $H^{r}$ is empty, then the rule is a propagation rule. At least one of $H^{r}$ and $H^{k}$ must be non-empty. We use $H_{i}$ to denote the heads of the $i$-th rule of a program. 


\subsubsection{Semantics: informal introduction by example}

A derivation starts from an initial query: a multiset of constraint atoms, given by the user. This multiset of constraints is called the constraint store. The derivation proceeds by applying the rules of the program, which modify the constraint store. When no more rules can be applied, the derivation ends; the final constraint store is called the solution or solved form.

Rules modify the constraint store in the following way. A simplification rule can be considered as a rewrite rule which replaces the left-hand side (the head constraints) with the right-hand side (the body constraints), on the condition that the guard holds. The double arrow indicates that the head is logically equivalent to the body, which justifies the replacement. The intention is that the body is a simpler, or more canonical form of the head.

In propagation rules, the body is a consequence of the head: given the head, the body may be added (if the guard holds). Logically, the body is implied by the head so it is redundant. However, adding redundant constraints may allow simplifications later on. Simpagation rules are a hybrid between simplification rules and propagation rules: the constraints before the backslash are kept, while the constraints after the backslash are removed.

Example 1 (less-than-or-equal). The CHR program LEQ is shown in Listing 3.1. It is a classic CHR program to solve less-than-or-equal constraints. The first rule, reflexivity, replaces the trivial constraint $\mathrm{leq}(\mathrm{X}, \mathrm{X})$ by true. Operationally, this entails removing this constraint from the constraint store (the multiset of all known CHR constraints). The second rule, antisymmetry, states that leq $(x, y)$ and leq $(y, x)$ are logically equivalent to $x=y$. Operationally this means that constraints matching the left-hand side may be removed from the store, after which the Prolog built-in equality constraint solver is used to unify $x$ and $y$. The third rule, idempotence, removes redundant copies of the same leq/2 constraint. It is necessary to do this explicitly since CHR has a multiset semantics. The last rule, transitivity, is a propagation rule that computes the transitive closure of the leq/ 2 relation.

An example derivation for the LEQ program would be the following:

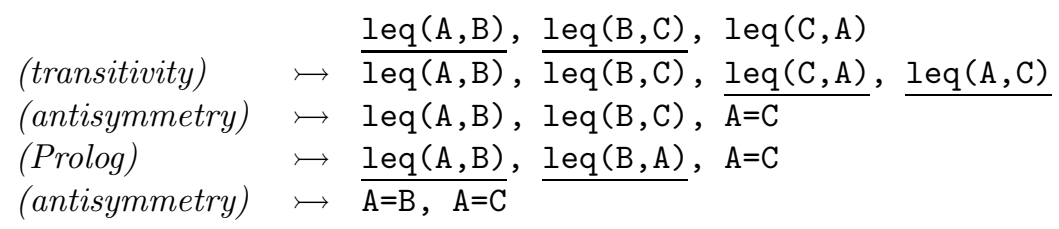

Starting from the same initial query, multiple derivations may be possible. For example, another derivation is the following: 
Listing 3.1: LEQ: Solver for the less-than-or-equal constraint

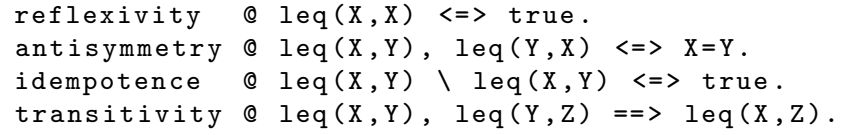

Listing 3.2: PRIMES: Prime number generator

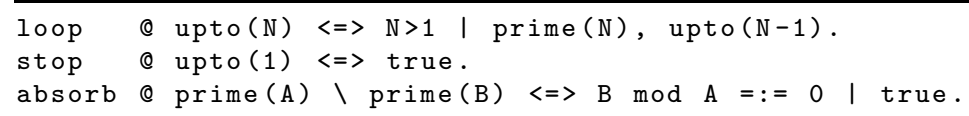

\begin{tabular}{|c|c|c|c|c|c|}
\hline (tran & $\zeta$ & $\begin{array}{l}\operatorname{leq}(A, B), \\
\operatorname{leq}(A, B),\end{array}$ & $\frac{\operatorname{leq}(B, C)}{\operatorname{leq}(B, C)}$, & $\frac{\operatorname{leq}(C, A)}{\operatorname{leq}(C, A)}$ & $\operatorname{leq}(B, A)$ \\
\hline (antisymmetry) & $\longmapsto$ & $\overline{\operatorname{leq}(B, C)}$, & $\operatorname{leq}(C, A)$ & $A=B$ & \\
\hline (Prolog) & $\longmapsto$ & $\operatorname{leq}(A, C)$, & $\operatorname{leq}(C, A)$, & $A=B$ & \\
\hline ntisymmetry) & $\longmapsto$ & $=\mathrm{C}, \mathrm{A}=\mathrm{B}$ & & & \\
\hline
\end{tabular}

In the case of the LEQ program, it can be shown that all derivations ultimately lead to the same result. If a program has this property, we say it is confluent. Confluence will be discussed in Section 3.2.1.

Example 2 (prime numbers). $\quad$ Listing 3.2 shows a simple CHR(Prolog) program called PRIMES, a CHR variant of the Sieve of Eratosthenes. Dating back to 1992 (Frühwirth 1992), this is one of the very first examples where CHR is used as a general-purpose programming language. Given a query of the form "upto $(n)$ ", where $n$ is a positive integer, it computes all prime numbers up to $n$. The first rule (loop) does the following: if $n>1$, it simplifies upto( $n$ ) to upto $(n-1)$ and adds a prime $(n)$ constraint. The second rule handles the case for $n=1$, removing any upto(1) constraint. Removing a constraint is done by simplifying it to the built-in constraint true. The third and most interesting rule (absorb) is a simpagation rule. If there are two prime/1 constraints prime $(a)$ and prime $(b)$, such that $b$ is a multiple of $a$, the latter constraint is removed. The effect of the absorb rule is that all non-primes are eventually removed. As a result, if the rules are applied exhaustively, the remaining constraints correspond exactly to the prime numbers up to $n$. 


\subsubsection{Logical semantics}

There are two ways to define the meaning of CHR programs (or declarative programming languages in general): a logical semantics formulates the meaning of a program in terms of a mapping to logical theories, while an operational semantics describes the behavior of a program, usually in terms of a state transition system that models program execution. Of course there should be a correspondence between both kinds of semantics. Soundness and completeness results, linking the logical semantics and the operational semantics, can be found in Frühwirth (1998).

In this section we describe the two main logical semantics of CHR: the original classical logic semantics, and the recent linear logic semantics, which was motivated by the shift towards general-purpose programming.

\section{Classical logic semantics}

Let $\bar{x}$ denote the variables occurring only in the body of the rule. A simplification rule $H \Longleftrightarrow G \mid B$ corresponds to a logical equivalence, under the condition that the guard is satisfied: $\forall(G \rightarrow(H \leftrightarrow \exists \bar{x} B))$. A propagation rule $H \Longrightarrow$ $G \mid B$ corresponds to a logical implication if the guard is satisfied: $\forall(G \rightarrow(H \rightarrow$ $\exists \bar{x} B)$ ). A simpagation rule $H_{k} \backslash H_{r} \Longleftrightarrow G \mid B$ corresponds to a conditional equivalence: $\forall\left(G \rightarrow\left(H_{k} \rightarrow\left(H_{r} \leftrightarrow \exists \bar{x} B\right)\right)\right)$. The (classical) logical semantics (Frühwirth 1998) of a CHR program - also called its logical reading, declarative semantics, or declarative interpretation - is given by the built-in constraint theory $\mathcal{D}_{\mathcal{H}}$ (which defines the built-ins of the host language $\mathcal{H}$ ) in conjunction with the logical formulas for each rule. As an example, consider again the program LEQ of Example 1. The logical formulas corresponding to its rules are the following:

$$
\left\{\begin{array}{lr}
\forall x, y: x=y \rightarrow(\operatorname{leq}(x, y) \leftrightarrow \operatorname{true}) & \text { (reflex.) } \\
\forall x, y, x^{\prime}, y^{\prime}: x=x^{\prime} \wedge y=y^{\prime} \rightarrow\left(\operatorname{leq}(x, y) \wedge \operatorname{leq}\left(y^{\prime}, x^{\prime}\right) \leftrightarrow x=y\right) & \text { (antisym.) } \\
\forall x, y, x^{\prime}, y^{\prime}: x=x^{\prime} \wedge y=y^{\prime} \rightarrow\left(\operatorname{leq}(x, y) \rightarrow\left(\operatorname{leq}\left(x^{\prime}, y^{\prime}\right) \leftrightarrow \text { true }\right)\right) & \text { (idempot.) } \\
\forall x, y, y^{\prime}, z: y=y^{\prime} \rightarrow\left(\operatorname{leq}(x, y) \wedge \operatorname{leq}\left(y^{\prime}, z\right) \rightarrow \operatorname{leq}(x, z)\right) & \text { (transit.) }
\end{array}\right.
$$

or equivalently:

$$
\left\{\begin{array}{lr}
\forall x: \operatorname{leq}(x, x) & \text { (reflexivity) } \\
\forall x, y: \operatorname{leq}(x, y) \wedge \operatorname{leq}(y, x) \leftrightarrow x=y & \text { (antisymmetry) } \\
\operatorname{true} & \text { (idempotence) } \\
\forall x, y, z: \operatorname{leq}(x, y) \wedge \operatorname{leq}(y, z) \rightarrow \operatorname{leq}(x, z) & \text { (transitivity) }
\end{array}\right.
$$

Note the strong correspondence between the syntax of the CHR rules, their logical reading, and the natural definition of partial order.

The classical logical reading, however, does not reflect CHR's multiset semantics (the idempotence rule is logically equivalent to true). Also, the classical logic reading does not always make sense. For example, consider the classical logic 
reading of the PRIMES program of Example 2

$$
\left\{\begin{array}{l}
\forall n: n>1 \rightarrow \operatorname{upto}(n) \leftrightarrow \exists n^{\prime} \operatorname{prime}(n) \wedge n^{\prime}=n-1 \wedge \text { upto }\left(n^{\prime}\right) \quad \text { (loop) } \\
\text { upto }(1) \leftrightarrow \text { true } \\
\forall a, b: b \bmod a=0 \rightarrow \operatorname{prime}(a) \rightarrow(\operatorname{prime}(b) \leftrightarrow \text { true })
\end{array}\right.
$$

which is equivalent to:

$$
\left\{\begin{array}{lr}
\forall n>1: \operatorname{upto}(n) \leftrightarrow \operatorname{prime}(n) \wedge \operatorname{upto}(n-1) & \text { (loop) } \\
\text { upto }(1) & \text { (stop) } \\
\forall a, b: \operatorname{prime}(a) \wedge b \bmod a=0 \rightarrow \operatorname{prime}(b) & \text { (absorb) }
\end{array}\right.
$$

The last formula nonsensically states that a number is prime if it has a prime factor.

\section{Linear logic semantics}

For general-purpose CHR programs such as PRIMES, or programs that rely on CHR's multiset semantics, the classical logic reading is often inconsistent with the intended meaning. To overcome these limitations, Bouissou (2004) and Betz and Frühwirth $(2005,2007)$ independently proposed an alternative declarative semantics based on (intuitionistic) linear logic. The latter, most comprehensive study provides strong soundness and completeness results, as well as a semantics for the $\mathrm{CHR}^{\vee}$ extension of CHR (see Section 3.4.1). For CHR programs whose constraints represent a multiset of resources, or whose rules represent unidirectional actions or updates, a linear logic semantics proves much more adequate. A simple example is the following coin-throwing simulator (which depends on the nondeterminism in the operational semantics):

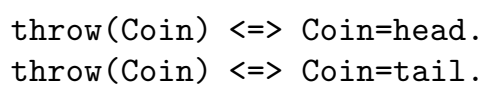

The classical logic reading of this program entails head $=$ tail. The linear logic reading of the coin-throwing program boils down to the following formula:

$$
!(\operatorname{throw}(\text { Coin }) \multimap(\text { Coin }=\text { head }) \&(\text { Coin = tail }))
$$

In natural language, this formula means "you can always replace throw(Coin) with either $($ Coin $=$ head $)$ or $($ Coin = tail $)$, but not both". This corresponds to the committed-choice and unidirectional rule application of CHR.

\subsubsection{Abstract operational semantics $\omega_{t}$}

In this section we present the (abstract) operational semantics $\omega_{t}$ of CHR, sometimes also called theoretical or high-level operational semantics.

The $\omega_{t}$ semantics is formulated as a state transition system. Transition rules define the relation between an execution state and its subsequent execution state. 
1. Solve. $\langle\{c\} \uplus \mathbb{G}, \mathbb{S}, \mathbb{B}, \mathbb{T}\rangle_{n} \longmapsto_{\mathcal{P}}\langle\mathbb{G}, \mathbb{S}, c \wedge \mathbb{B}, \mathbb{T}\rangle_{n}$ where $c$ is a built-in constraint and $\mathcal{D}_{\mathcal{H}} \models \bar{\exists}_{\emptyset} \mathbb{B}$.

2. Introduce. $\langle\{c\} \uplus \mathbb{G}, \mathbb{S}, \mathbb{B}, \mathbb{T}\rangle_{n} \longmapsto_{\mathcal{P}}\langle\mathbb{G},\{c \# n\} \cup \mathbb{S}, \mathbb{B}, \mathbb{T}\rangle_{n+1}$ where $c$ is a $\mathrm{CHR}$ constraint and $\mathcal{D}_{\mathcal{H}} \models \bar{\exists}_{\emptyset} \mathbb{B}$.

3. Apply. $\left\langle\mathbb{G}, H_{1} \cup H_{2} \cup \mathbb{S}, \mathbb{B}, \mathbb{T}\right\rangle_{n} \longmapsto_{\mathcal{P}}\left\langle C \uplus \mathbb{G}, H_{1} \cup \mathbb{S}, \theta \wedge \mathbb{B}, \mathbb{T} \cup\{h\}\right\rangle_{n}$ where $\mathcal{P}$ has a (renamed apart) rule of the form $r @ H_{1}^{\prime} \backslash H_{2}^{\prime} \Longleftrightarrow G \mid C$, $\theta$ is a matching substitution with $\operatorname{chr}\left(H_{1}\right)=\theta\left(H_{1}^{\prime}\right)$ and $\operatorname{chr}\left(H_{2}\right)=\theta\left(H_{2}^{\prime}\right)$, $h=\left(r, i d\left(H_{1}\right), i d\left(H_{2}\right)\right) \notin \mathbb{T}$, and $\mathcal{D}_{\mathcal{H}} \models\left(\bar{\exists}_{\emptyset} \mathbb{B}\right) \wedge\left(\mathbb{B} \rightarrow \bar{\exists}_{\mathbb{B}}(\theta \wedge G)\right)$.

Figure 3.1: Transitions of the abstract (theoretical) operational semantics $\omega_{t}$

Definition 3.1 (identified CHR constraint) An identified CHR constraint $c \# i$ is a CHR constraint $c$ associated with some unique integer $i$, called the constraint identifier. This number serves to differentiate among copies of the same constraint. We introduce the functions $\operatorname{chr}(c \# i)=c$ and $i d(c \# i)=i$, and extend them to sequences and sets of identified CHR constraints in the obvious manner, e.g. $i d(\mathbb{S})=\{i \mid c \# i \in \mathbb{S}\}$. Note that $\operatorname{chr}(\mathbb{S})$ is a multiset although $\mathbb{S}$ is a set.

Definition 3.2 (execution state) An execution state $\sigma$ is a tuple $\langle\mathbb{G}, \mathbb{S}, \mathbb{B}, \mathbb{T}\rangle_{n}$. The goal $\mathbb{G} \in \mathcal{G}_{\mathcal{P}}^{\mathcal{H}}$ is a multiset of constraints to be rewritten to solved form. The CHR constraint store $\mathbb{S}$ is a set of identified CHR constraints that can be matched with rules in the program $\mathcal{P}$. The built-in constraint store $\mathbb{B}$ is the conjunction of all built-in constraints that have been passed to the underlying solver. This abstracts the internal representation used by the host language. Its actual meaning depends on the host language $\mathcal{H}$. The propagation history $\mathbb{T}$ is a set of tuples, each recording the identities of the CHR constraints that fired a rule, and the name of the rule itself. It is used to prevent trivial non-termination for propagation rules: a propagation rule is allowed to fire on a set of constraints only if the constraints have not been used to fire the same rule before. Finally, the counter $n \in \mathbb{N}$ represents the next free integer that can be used to identify a CHR constraint.

We use $\sigma, \sigma_{0}, \sigma_{1}, \ldots$ to denote execution states and $\Sigma^{\mathrm{CHR}}$ to denote the set of all states. Transitions are defined by the binary relation $\longmapsto_{\mathcal{P}}: \Sigma^{\mathrm{CHR}} \rightarrow \Sigma^{\mathrm{CHR}}$ shown in Figure 3.1. Execution proceeds by exhaustively applying the transitions, starting from an initial state. We define $\succ_{\mathcal{P}}^{*}$ as the transitive closure of $\longmapsto \mathcal{P}$.

\subsubsection{Derivations}

Definition 3.3 (initial state) Given an initial goal (query) $\mathbb{G} \in \mathcal{G}_{\mathcal{P}}^{\mathcal{H}}$, we define initstate $(\mathbb{G})=\langle\mathbb{G}, \emptyset \text {, true, } \emptyset\rangle_{1}$. The set of initial states is denoted by $\Sigma^{\text {init }}$. 
Definition 3.4 (final state) A final state $\sigma_{f}=\langle\mathbb{G}, \mathbb{S}, \mathbb{B}, \mathbb{T}\rangle_{n}$ is an execution state for which no transition applies: $\neg \exists \sigma \in \Sigma^{\mathrm{CHR}}: \sigma_{f} \longmapsto_{\mathcal{P}} \sigma$. In a failure state, the underlying solver $\mathcal{H}$ can prove $\mathcal{D}_{\mathcal{H}} \models \neg \bar{\exists}_{\emptyset} \mathbb{B}$ - such states are always final. $A$ successful final state is a final state that is not a failure state, i.e. $\mathcal{D}_{\mathcal{H}}=\bar{\exists}_{\emptyset} \mathbb{B}$. The set of final states is denoted by $\Sigma^{\text {final }} \subset \Sigma^{\mathrm{CHR}}$.

Definition 3.5 (finite derivation) Given a $C H R$ program $\mathcal{P}$, a finite derivation $d$ is a finite sequence $\left[\sigma_{0}, \sigma_{1}, \ldots, \sigma_{n}\right]$ of states where $\sigma_{0} \in \Sigma^{\text {init }}, \sigma_{n} \in \Sigma^{\text {final }}$, and $\sigma_{i} \longmapsto_{\mathcal{P}} \sigma_{i+1}$ for $0 \leq i<n$. If $\sigma_{n}$ is a failure state, we say $d$ has failed, otherwise $d$ is a successful derivation.

Programs do not necessarily terminate, so derivations are not always finite:

Definition 3.6 (infinite derivation) An infinite derivation $d_{\infty}$ is an infinite sequence $\sigma_{0}, \sigma_{1}, \ldots$ of states where $\sigma_{0} \in \Sigma^{\text {init }}$ and $\sigma_{i} \longmapsto_{\mathcal{P}} \sigma_{i+1}$ for $i \in \mathbb{N}$.

Two syntactically different execution states are essentially the same if they are renamings of another or if they are both failure states. We say they are variants:

Definition 3.7 (variant states) Two execution states $\sigma=\langle\mathbb{G}, \mathbb{S}, \mathbb{B}, \mathbb{T}\rangle_{n}$ and $\sigma^{\prime}=$ $\left\langle\mathbb{G}^{\prime}, \mathbb{S}^{\prime}, \mathbb{B}^{\prime}, \mathbb{T}^{\prime}\right\rangle_{n^{\prime}}$ are variants if and only if 1) a variable renaming $\theta$ exists such that $\operatorname{chr}(\mathbb{S})=\operatorname{chr}\left(\theta\left(\mathbb{S}^{\prime}\right)\right)$ and $\mathcal{D}_{\mathcal{H}} \models \mathbb{B} \leftrightarrow \theta\left(\mathbb{B}^{\prime}\right)$, or 2) $\mathcal{D}_{\mathcal{H}} \models \neg \bar{\exists}_{\emptyset} \mathbb{B} \wedge \neg \bar{\exists}_{\emptyset} \mathbb{B}^{\prime}$ (both states are failure states). We denote this as follows: $\sigma \approx \sigma^{\prime}$.

We use \#d to denote the length of a derivation: the length of a finite derivation is the number of transitions in the sequence; the length of an infinite derivation is $\infty$. A set of (finite or infinite) derivations is denoted by $\Delta$. The set of all derivations in $\Delta$ that start with initstate $(\mathbb{G})$ is denoted by $\left.\Delta\right|_{\mathbb{G}}$. We use $\Delta_{\omega_{t}}^{\mathcal{H}}(\mathcal{P})$ to denote the set of all derivations (in the $\omega_{t}$ semantics) for a given CHR program $\mathcal{P}$ and host language $\mathcal{H}$. We now define the relation $\rightsquigarrow_{\Delta}: \Sigma^{\text {init }} \rightarrow \Sigma^{\mathrm{CHR}} \cup\{\infty\}$ :

Definition 3.8 ( $\Delta$-output) State $\sigma_{n}$ is a $\Delta$-output of $\sigma_{0}$ if $\left[\sigma_{0}, \ldots, \sigma_{n}\right] \in \Delta$. We say $\sigma_{0} \Delta$-outputs $\sigma_{n}$ and write $\sigma_{0} \rightsquigarrow \Delta \sigma_{n}$. If $\Delta$ contains an infinite derivation starting with $\sigma_{0}$, we say $\sigma_{0}$ has a non-terminating derivation. We denote this as follows: $\sigma_{0} \rightsquigarrow \Delta \infty$.

Definition 3.9 ( $\Delta$-deterministic) The $C H R$ program $\mathcal{P}$ is $\Delta$-deterministic for input $I \subseteq \mathcal{G}_{\mathcal{P}}^{\mathcal{H}}$ if the restriction of $\rightsquigarrow \Delta$ to initstate $(I)$ is a function, modulo variant states (so for any input in I, all output states are variants of each other), and $\forall i \in I,\left.d \in \Delta\right|_{i}:$ if $d$ is a successful derivation, then $\left.\forall d^{\prime} \in \Delta\right|_{i}: \# d=\# d^{\prime}$.

In other words, a program is $\Delta$-deterministic if all derivations starting from a given input have the same result and all successful ones have the same length. 
(1) Note that if a program $\mathcal{P}$ is $\Delta_{\omega_{t}}^{\mathcal{H}}(\mathcal{P})$-deterministic for all input $\mathcal{G}_{\mathcal{P}}^{\mathcal{H}}$, it is also observable confluent (Duck, Stuckey, and Sulzmann 2007). The notion of observable confluence does not require derivations to have the same length.

Example 3 (PRIMES is $\Delta_{\omega_{t}}^{\text {Prolog }}$-deterministic). Consider the CHR program

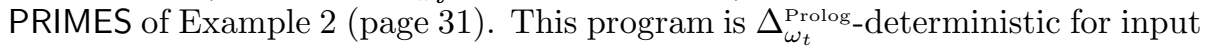
$\{$ upto $(n) \mid n \in \mathbb{N}\}$. Although the order in which the transitions of $\omega_{t}$ are applied is not fixed for a given input, allowing different derivations, the derivation length and result is always the same.

Of course not every CHR program is $\Delta_{\omega_{t}}^{\mathcal{H}}$-deterministic for its intended input:

Example 4 (LEQ is not $\Delta_{\omega_{t}}^{\text {Prolog }}$-deterministic). The CHR program LEQ of Example 1 (page 31) is not $\Delta_{\omega_{t}}^{\text {Prolog }}$-deterministic for all conjunctions of leq/2 constraints. Consider the input query "leq(A,B), leq $(B, A)$ ". One derivation consists of two Introduce steps followed by an Apply step using the antisymmetry rule, followed by a Solve step for "A = B" and two Apply steps using the reflexivity rule. This derivation has length six. In another derivation, the transitivity rule is applied after the Introduce steps. This results in a longer derivation, or even an infinite derivation.

For the connoisseurs: LEQ is not even $\Delta_{\omega_{r}}^{\text {Prolog }}$-deterministic (i.e. restricting the derivations to those of the refined operational semantics $\omega_{r}$, cf. Section 4.3). Given the query "leq $(B, C)$, leq $(B, A)$, leq $(A, B)$ ", the derivation length depends on which partner for $\operatorname{leq}(A, B)$ is tried first when the transitivity rule is applied.

\subsection{Program properties}

\subsubsection{Confluence}

If for a given CHR program, for all initial states, any $\omega_{t}$ derivation from that state results in the same final state, the program is called confluent (Abdennadher, Frühwirth, and Meuss 1999). Confluence implies correctness, in the sense that the logical reading is consistent for confluent programs (Frühwirth 1998).

Confluence is defined in terms of joinable execution states:

Definition 3.10 (joinable states) Execution states $\sigma_{1}$ and $\sigma_{2}$ are joinable if there exist states $\sigma_{1}^{\prime}$ and $\sigma_{2}^{\prime}$ such that $\sigma_{1} \longmapsto_{\mathcal{P}}^{*} \sigma_{1}^{\prime}$ and $\sigma_{2} \longmapsto_{\mathcal{P}}^{*} \sigma_{2}^{\prime}$ and $\sigma_{1}^{\prime} \approx \sigma_{2}^{\prime}$.

Definition 3.11 (confluence) A CHR program $\mathcal{P}$ is confluent if, for every initial state $\sigma \in \Sigma^{\text {init }}$, the following holds: if $\sigma \longmapsto_{\mathcal{P}}^{*} \sigma_{1}$ and $\sigma \longmapsto_{\mathcal{P}}^{*} \sigma_{2}$, then $\sigma_{1}$ and $\sigma_{2}$ are joinable. 
The above definition deviates slightly from the usual definitions in the literature: normally every execution state is considered instead of only the initial states. There are programs that are confluent according to the above definition but not according to the usual definition, where non-confluence originates from unreachable states. This issue has been addressed in a general way by introducing the notion of observable confluence (Duck, Stuckey, and Sulzmann 2007).

There is a decidable, sufficient and necessary test for confluence (according to the usual definition) of terminating programs (Frühwirth 1998). Recently, the topic of confluence received renewed attention because certain problems and limitations of this confluence test have surfaced. Firstly, many programs that are in practice confluent fail the test because non-confluence originates from unreachable states. The framework of observable confluence (Duck, Stuckey, and Sulzmann 2007) allows a restriction to reachable states. Secondly, the test is only applicable to terminating programs. Raiser and Tacchella (2007) investigated confluence of non-terminating programs.

Confluence under the refined operational semantics (see Section 4.3) is investigated in Chapter 6 of (Duck 2005), which also discusses a refined confluence checker.

Completion. Abdennadher and Frühwirth (1998) showed how to do completion of CHR programs. Completion is a technique to transform a non-confluent program into a confluent one by adding rules. It allows extension, modification and specialization of existing programs.

Operational equivalence. A very useful notion is that of operational equivalence: do two CHR programs have the same input-output behavior. Programs $\mathcal{P}_{1}$ and $\mathcal{P}_{2}$ are defined to be operationally equivalent if for every execution state $\sigma$, there are derivations $\sigma \longmapsto_{\mathcal{P}_{1}}^{*} \sigma_{1}$ and $\sigma \succ_{\mathcal{P}_{2}}^{*} \sigma_{2}$ such that $\sigma_{1} \approx \sigma_{2}$. Abdennadher and Frühwirth (1999) have given a decidable, sufficient, and necessary syntactic condition for operational equivalence of well-behaved (confluent and terminating) CHR programs.

\subsubsection{Termination}

The first work on termination analysis of CHR programs was presented by Frühwirth (2000). Frühwirth demonstrated that termination proof techniques from logic programming and term rewrite systems can be adapted to the CHR context.

Termination of CHR programs is proved by defining a ranking function from computation states to a well-founded domain such that the rank of consecutive computation states decreases. A condition on simplification rules guarantees such rank decreases for all consecutive states. This approach, however, cannot prove termination of CHR programs with propagation rules, because it is impossible to 
show decreases between consecutive states as these rules do not remove constraints from the store.

Recent termination analyses by Pilozzi et al. $(2007,2008)$ and Voets et al. (2007) work on a much larger class of CHR programs and are able to deal with programs that contain both simplification and propagation rules.

\subsubsection{Complexity}

For various CHR programs an accurate ad hoc complexity analysis has been made. In Chapter 5 we discuss some examples of such complexity analyses. Two other recent examples are the work of Frühwirth (2005a), who formulated the complexity of a general-purpose lexicographical order constraint solver in terms of the number of ask and tell built-in constraints encountered during execution, and that of Meister et al. (2006), who derive the complexity of a solver for existentially quantified equations over finite and infinite trees, using bounds on the derivation length.

While ad hoc methods give the most accurate results in practice, they cannot easily be generalized. Therefore, more structured approaches to complexity analysis have been proposed by means of meta-complexity theorems.

Frühwirth $(2001,2002 \mathrm{a}, 2002 \mathrm{~b})$ investigated the time complexity of simplification rules for naive implementations of CHR. In this approach, a suitable termination order (called a tight ranking) is used as an upper bound on the derivation length. Combined with a worst-case estimate of the number and cost of rule application attempts, this results in a complexity meta-theorem which gives a rough upper bound of the time complexity. As we will see, these bounds are rather crude. Recent work on optimizing compilation of CHR - which we will discuss in Chapter 4 and Part II - allows meta-theorems that give much tighter complexity bounds. In Part III, in particular in Chapter 11, we will prove a stronger complexity meta-theorem.

(-) Another approach is the following. Ganzinger and McAllester (2002) proposed a formalism called Logical Algorithms (LA) and proved a meta-complexity result for LA. De Koninck et al. (2007a) establish a close correspondence between CHR and LA (see also Section 3.7.2), allowing the LA meta-complexity result to be applied (indirectly) to a large class of CHR programs. De Koninck et al. (2007a) actually address the meta-complexity of $\mathrm{CHR}^{\mathrm{rp}}$ programs, an extension of CHR discussed in Section 4.1.4. All CHR programs are also $\mathrm{CHR}^{\mathrm{rp}}$ programs. The Logical Algorithms approach was previously used, in an ad hoc way, by Christiansen (2005a) to derive the complexity of CHR grammars (see Section 3.6.5).

\subsection{CHR systems}

Since the conception of CHR, a large number of CHR systems (compilers, interpreters and ports) have been developed. In particular, in the last ten years the 
number of systems has exploded. Figure 3.2 presents a timeline of system development, branches and influences. In this section we briefly discuss these systems, grouped by host language paradigm.

\subsubsection{CHR(LP)}

Logic Programming is a natural (or at least traditional) host language paradigm for CHR. Hence, it is not surprising that the CHR(Prolog) implementations are the most established ones. Holzbaur and Frühwirth (2000a) have laid the groundwork with their general compilation scheme for Prolog. This compilation scheme ${ }^{1}$ was first implemented in SICStus Prolog by Holzbaur, and later further refined in HAL by Holzbaur et al. (2005) and in hProlog by Schrijvers and Demoen (2004). Another system directly based on the work of Holzbaur and Schrijvers is the CHR library for SiLCC by Bouissou (2004). SiLCC is a programming language based on linear logic and concurrent constraint programming. All of these systems compile CHR programs to host language programs. The only available interpreter for $\mathrm{CHR}\left(\right.$ Prolog) is TOYCHR ${ }^{2}$.

\subsubsection{CHR(FP)}

As type checking is one of the most successful applications of CHR in the context of Functional Programming (see section 3.6.3), several CHR implementations were developed specifically for this purpose. Most notable is the Chameleon system (Stuckey and Sulzmann 2005) which features CHR as the programming language for its extensible type system. Internally, Chameleon uses the HaskellCHR implementation $^{3}$. The earlier HCHR prototype (Chin, Sulzmann, and Wang 2003) had a rather heavy-weight and impractical approach to logical variables.

The aim of a 2007 Google Summer of Code project was to transfer this CHR based type checking approach to two Haskell compilers (YHC and nhc98). The project led to a new CHR interpreter for Haskell, called TaiChi (Boespflug 2007).

With the advent of software transactional memories (STM) in Haskell, two prototype systems with parallel execution strategies have been developed: $\mathrm{STMCHR}^{4}$ and Concurrent CHR (Lam and Sulzmann 2007). These systems are currently the only CHR implementations that exploit the inherent parallelism in CHR programs.

We also mention the Haskell library for the PAKCS implementation of the functional logic language Curry (Hanus 2006). The PAKCS system actually compiles Curry code to SICStus Prolog, and its CHR library is essentially a front-end for the SICStus Prolog CHR library. The notable added value of the Curry front-end is the (semi-)typing of the CHR code.

\footnotetext{
${ }^{1}$ The compilation scheme will be explained in some detail in Chapter 4 .

${ }^{2}$ by Gregory J. Duck, 2003. Download: http://www.cs.mu.oz.au/ gjd/toychr/

${ }^{3}$ by Gregory J. Duck, 2004. Download: http://www.cs.mu.oz.au/ gjd/haskellchr/

${ }^{4}$ by Michael Stahl, 2007. Download: http://www.cs.kuleuven.be/ dtai/projects/CHR/
} 


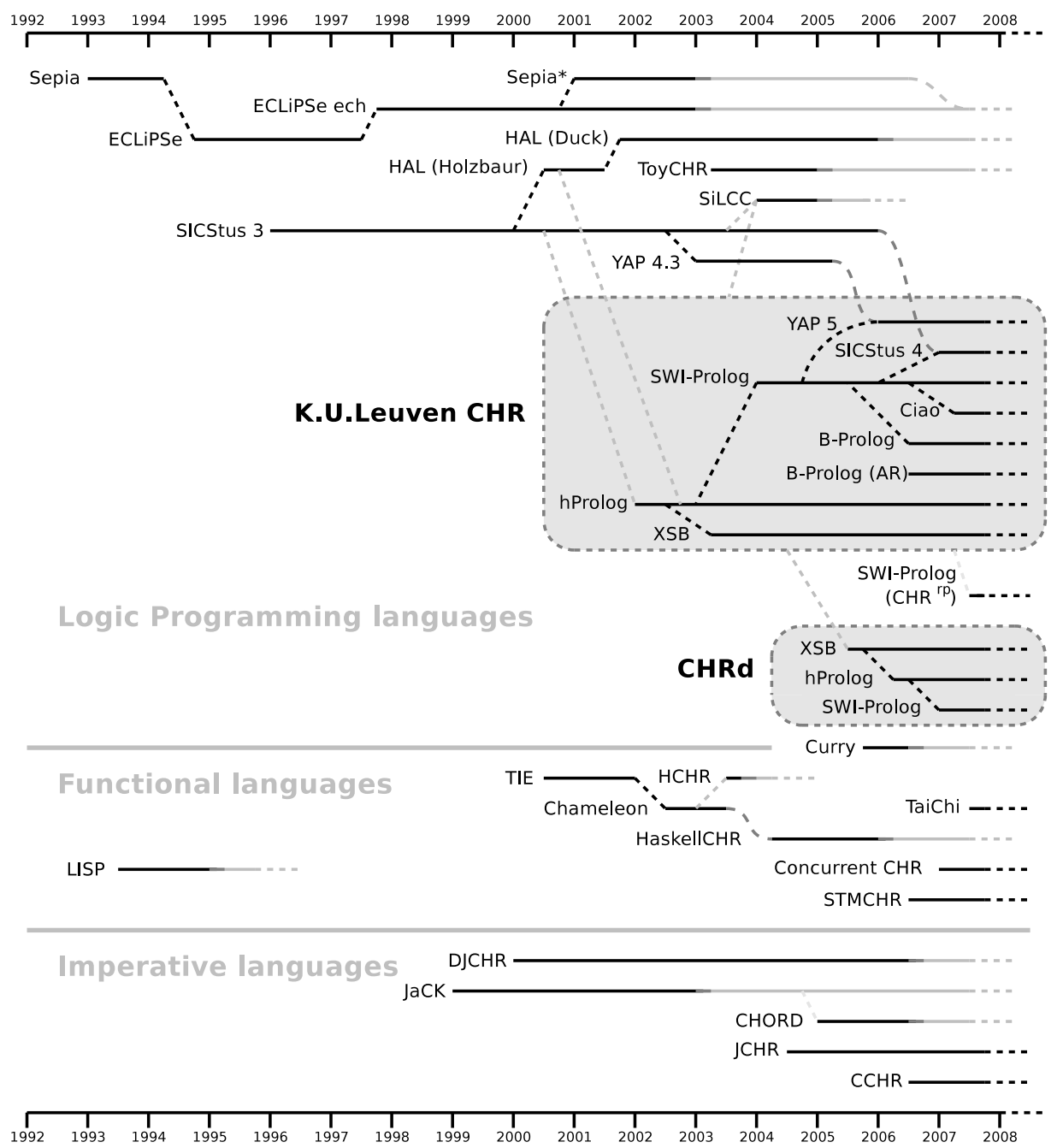

Figure 3.2: Timeline of CHR systems 


\subsubsection{CHR(Java) and $\mathrm{CHR}(\mathrm{C})$}

Finally, CHR systems are also available for both Java and C. For a detailed discussion on the different conceptual and technical challenges encountered when embedding CHR into an imperative host language, see (Van Weert, Wuille, Schrijvers, and Demoen 2008).

CHR(Java). The earliest CHR(Java) system is the Java Constraint Kit (JaCK) by Abdennadher (2001) and others (Abdennadher, Krämer, Saft, and Schmauß 2002; Schmauß 1999). DJCHR (Dynamic JCHR; Wolf 2001a) is an implementation of adaptive CHR (see Section 3.4.3). The incremental adaptation algorithm underlying DJCHR maintains justifications for rule applications and constraint additions. The Leuven JCHR system ${ }^{5}$ (Van Weert, Schrijvers, and Demoen 2005) focusses on performance and integration with the host language. It is currently one of the most efficient CHR systems available. A fourth CHR(Java) system is called CHORD (Constraint Handling Object-oriented Rules with Disjunctive bodies) ${ }^{6}$.

CHR(C). CCHR (Wuille, Schrijvers, and Demoen 2007) implements CHR for C. It is an extremely efficient CHR system and uses a syntax that is intuitive to both CHR adepts and imperative programmers.

\subsubsection{The Leuven CHR system}

The Leuven CHR system ${ }^{7}$ is a state-of-the-art CHR system, developed by Tom Schrijvers and others at the K.U.Leuven. It is available in seven different Prolog systems: hProlog $^{8}$ (Schrijvers and Demoen 2004), SWI-Prolog 9 (Schrijvers, Wielemaker, and Demoen 2005), $\mathrm{XSB}^{10}$ (Schrijvers, Warren, and Demoen 2003; Schrijvers and Warren 2004), YAP ${ }^{11}$, B-Prolog ${ }^{12}$ (Schrijvers, Zhou, and Demoen 2006), SICStus Prolog ${ }^{13}$, and Ciao Prolog ${ }^{14}$.

Most of the work presented in this dissertation was done in the context of the Leuven CHR system: the compiler optimizations of Part II were implemented and tested in the system and the complexity results of Part III were experimentally verified in the system.

\footnotetext{
${ }^{5}$ Leuven JCHR system home page: http://www.cs.kuleuven.be/ petervw/JCHR/

${ }^{6}$ by Jairson Vitorino and Marcos Aurelio, 2005, http://chord.sourceforge.net/

${ }^{7}$ Leuven CHR system home page: http://www.cs.kuleuven.be/ toms/Research/CHR/

${ }^{8}$ by Bart Demoen, http://www.cs.kuleuven.be/ bmd/hProlog/

${ }^{9}$ by Jan Wielemaker, http://www.swi-prolog.org/

${ }^{10}$ by David S. Warren et al, http://xsb.sf .net/

${ }^{11}$ by Vítor Santos Costa et al, http://www.ncc.up.pt/ vsc/Yap/

12 by Neng-Fa Zhou, http://www.probp.com

${ }^{13}$ by Mats Carlsson et al, http://www.sics.se/isl/sicstuswww/site/

${ }^{14}$ by Manuel Hermenegildo et al, http://www.ciaohome.org/
} 
One of the distinguishing features of the Leuven CHR system is the option to add type and mode declarations of constraint arguments. We discuss these declarations in Section 3.5.1 and they will be used throughout the text.

\subsection{Extensions of CHR}

Over the years, some additional language features have been proposed for CHR. In this section we give a brief overview of some of them.

\subsubsection{Disjunction and search}

Most constraint solvers require search next to constraint simplification and propagation. However pure CHR does not offer any support for search. Abdennadher and Schütz (1998) propose a solution to this problem: an extension of CHR with disjunctions in rule bodies (see also Abdennadher 2000, 2001). The resulting language is denoted $\mathrm{CHR}^{\vee}$ (pronounced "CHR-or"). Any (pure) Prolog program can be rephrased as an equivalent $\mathrm{CHR}^{\vee}$ program (Abdennadher 2000, 2001). Implementation-wise, disjunction comes for free in CHR(Prolog) by means of the built-in Prolog disjunction and search mechanism.

In CHR(Java) systems, unlike in CHR(LP) systems, the host language does not provide search capabilities. The flexible specification of intelligent search strategies has therefore received considerable attention in several CHR(Java) systems (Krämer 2001; Wolf 2005). In these systems, the search strategies are implemented and specified in the host language itself, orthogonally to the actual CHR program.

(1) Various ways have been proposed to make the search in $\mathrm{CHR}^{\vee}$ programs more flexible and efficient. Menezes et al. (2005) present a $\mathrm{CHR}^{\vee}$ implementation for Java in which the search tree is made explicit and manipulated at runtime to improve efficiency. The nodes in the search tree can be reordered to avoid redundant work. De Koninck et al. (2006b) extend both the theoretical and refined operational semantics of $\mathrm{CHR}$ towards $\mathrm{CHR}^{\vee}$. The theoretical version leaves the search strategy undetermined, whereas the refined version allows the specification of various search strategies. In the same work, an implementation for different strategies in $\mathrm{CHR}$ (Prolog) is realized by means of a source-to-source transformation.

\subsubsection{Negation and aggregates}

CHR programmers often want to test for the absence of constraints. CHR was therefore extended with negation as absence by Van Weert et al. (2006). Negation as absence was later generalized to a much more powerful language feature, called aggregates (Sneyers, Van Weert, and Schrijvers 2007). Aggregates accumulate information over unbounded portions of the constraint store. Predefined 
aggregates include sum, count, findall, and min. The proposed extension also features nested aggregate expressions over guarded conjunctions of constraints, and application-tailored user-defined aggregates. Aggregates lead to increased expressivity and more concise programs. An implementation based on source-tosource transformations (Van Weert, Sneyers, and Demoen 2008) is available. The implementation uses efficient incremental aggregate computation, and empirical results show that the desired runtime complexity is attainable with an acceptable constant time overhead.

As an example of nested aggregate expressions, consider the following rule:

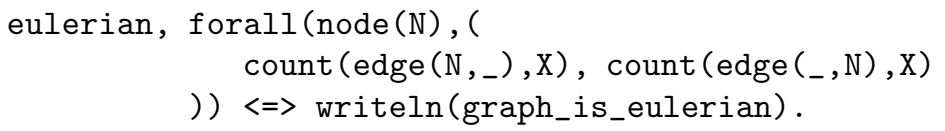

The above rule is applicable if for every constraint node $(\mathrm{N})$, the number of outgoing edges in $\mathrm{N}$ equals the number of incoming edges (i.e. if the first number is $\mathrm{X}$, the other number must also be $\mathrm{X}$ ).

\subsubsection{Adaptive CHR}

Constraint solving in a continuously changing, dynamic environment often requires immediate adaptation of the solutions, i.e. when constraints are added or removed. By nature, CHR solvers already support efficient adaptation when constraints are added. Wolf $(1999,2000)$ introduces an extended incremental adaptation algorithm which is capable of adapting CHR derivations after constraint deletions as well. This algorithm is further improved by Wolf (2000a) with the elimination of local variables using early projection. An efficient implementation exists in Java (Wolf 2001a, 2001b; cf. Section 3.3.3).

\subsubsection{Solver hierarchies}

While the theory of the CHR language generally considers arbitrary built-in solvers, traditional CHR implementations restrict themselves to the Herbrand equality constraint solver, with very little, if any, support for other constraint solvers.

Duck et al. (2003) show how to build CHR solvers on top of arbitrary built-in constraint solvers by means of ask constraints. The ask constraints signal the CHR solver when something has changed in the built-in store with respect to variables of interest. Then the relevant CHR constraints may be reactivated.

Schrijvers et al. (2006) provide an automated means for deriving ask versions of CHR constraints. In the approach of Fages et al. (2008), which is called CHRat, the programmer is supposed to (manually) implement both ask and tell versions of each constraint. Both approaches are aimed at adding a form of modularity to CHR. In this way full hierarchies of constraint solvers can be written, where one solver serves as the built-in solver for another solver. 


\subsection{Example CHR programs}

In this section we present some examples of CHR programs. Some of these examples will be reconsidered in later chapters, for instance for benchmarking purposes.

\subsubsection{Programming in CHR, in practice}

Before we discuss the examples, we first explain how to try these examples in practice, in the Leuven CHR system. Every CHR(Prolog) program starts by loading the CHR library:

:- use_module(library (chr)).

In program listings we do not explicitly include this line. Next, we have to declare which predicates are CHR constraints. The keyword chr_constraint is used for these declarations. For example, if the only CHR constraints used in a program are called foo, with two arguments, and bar, with three arguments, we need the following declaration:

:- chr_constraint foo/2, bar/3.

If a rule head contains an undeclared constraint, the Leuven CHR system produces an error message. In program listings we sometimes omit the constraint declarations, since they can easily be reconstructed by looking at the rule heads.

\section{Mode declarations}

The Leuven CHR system also supports a more advanced way to declare constraints. Instead of only giving the arity of each constraint predicate, the programmer can also provide the mode of each constraint argument. The default mode is "?", which corresponds to arbitrary argument instantiation. If an argument has mode "+", it should correspond to a ground term. The mode "-" indicates that the argument is an unbound variable. A typical example is the following:

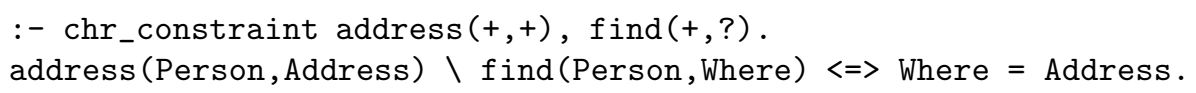

Mode declarations are crucial in several compiler optimizations; often performance can significantly be boosted by adding accurate mode declarations.

\section{Type declarations}

Even more information about the constraint arguments can optionally be declared by means of type declarations. The default type is any; other built-in types are int, float, number, and natural. User-defined types can be declared using the 
Listing 3.3: SUM: Sum of the elements of a list

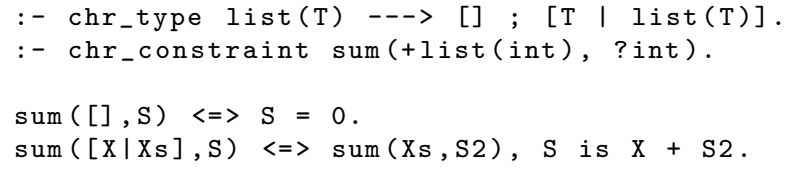

keyword chr_type. Algebraic data type in the style of Haskell or Mercury are supported. The syntax for type definitions is "type ---> body", where the right hand side is a disjunction of the different constructors. Type aliases can be defined using "==". We give some examples of type definitions:

\section{Example 5 (type definitions).}

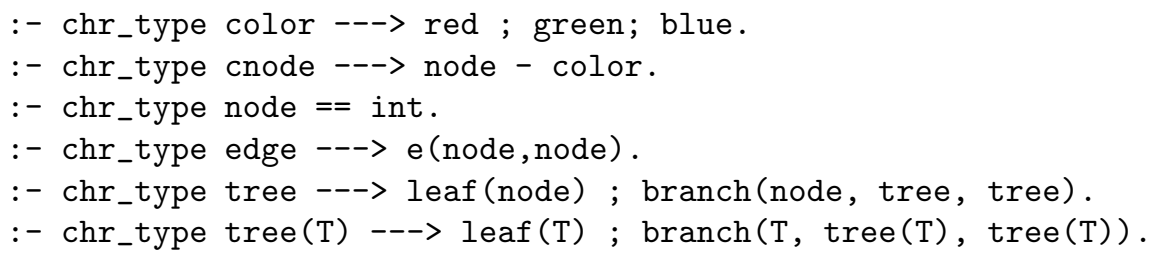

The Leuven CHR system performs both static and dynamic type-checking. Type information is also used in some compiler optimizations. As a simple example, consider the program SUM of Listing 3.3. Erroneous queries like sum $([4,7, \mathrm{x}], \mathrm{S})$ are detected by dynamic type checks, and an error message is given:

ERROR: Type error: 'int' expected, found ' $x$ ' (CHR Runtime Type Error)

Static type checking is useful for the early detection of bugs. For example, suppose there was a typo in the last rule:

$\operatorname{sum}([X \mid X s], S) \Leftrightarrow \operatorname{sum}(X s, S 2), S$ is $X+S 2$.

Now the first argument in the sum/2 constraint in the body becomes an atom xs instead of the variable Xs representing the tail of the list. The compiler will produce the following error message:

CHR compiler TYPE ERROR:

'--> Invalid functor in body goal sum(xs, _G22110)

of rule number 2 (line 7 ):

found ' $x s^{\prime}$ ',

expected type 'Iist(int)'! 
Listing 3.4: FIBONACCI: Top-down computation of Fibonacci numbers

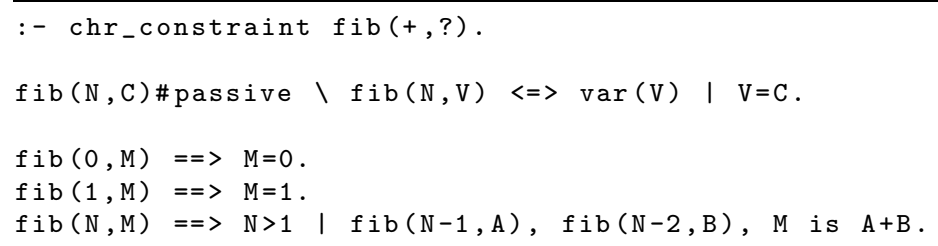

\subsubsection{Fibonacci numbers}

The Fibonacci numbers are named after the medieval Italian mathematician Leonardo of Pisa, also known as Fibonacci. The sequence is recursively defined as follows:

$$
\operatorname{fib}(n)= \begin{cases}0 & \text { if } n=0 \\ 1 & \text { if } n=1 \\ \operatorname{fib}(n-1)+\operatorname{fib}(n-2) & \text { if } n>1\end{cases}
$$

In other words, every Fibonacci number is the sum of the two previous Fibonacci numbers. The sequence starts as follows: $0,1,1,2,3,5,8,13,21,34,55,89, \ldots$

Example 6 (Fibonacci numbers). The program FIBONACCI, shown in Listing 3.4, can be used to compute Fibonacci numbers. Starting with a query of the form $\mathrm{fib}(n, \mathrm{Q})$, all Fibonacci numbers up to the $n$-th are computed and $\mathrm{Q}$ gets the value $\mathrm{fib}(n)$.

(1) The first rule is not strictly needed: however, if it is left out, the complexity of the program becomes exponential in $n$. The effect of the first rule is to reuse values that are already computed. This reduces the complexity to linear. See Section 4.3.2 for an explanation of the \#passive pragma; for now it may be ignored.

\subsubsection{Zebra puzzle}

The so-called zebra puzzle is a well-known logic puzzle, often attributed to Albert Einstein. Given five houses in a row, painted in different colors, the puzzle is to figure out who lives where, drinks what drink, smokes which brand of cigarettes and owns which pet. The following clues are given:

1. The Englishman lives in the red house.

2. The Spaniard owns the dog.

3. Coffee is drunk in the green house.

4. The Ukrainian drinks tea. 
Listing 3.5: ZEBRA: Solver for puzzles like the zebra puzzle

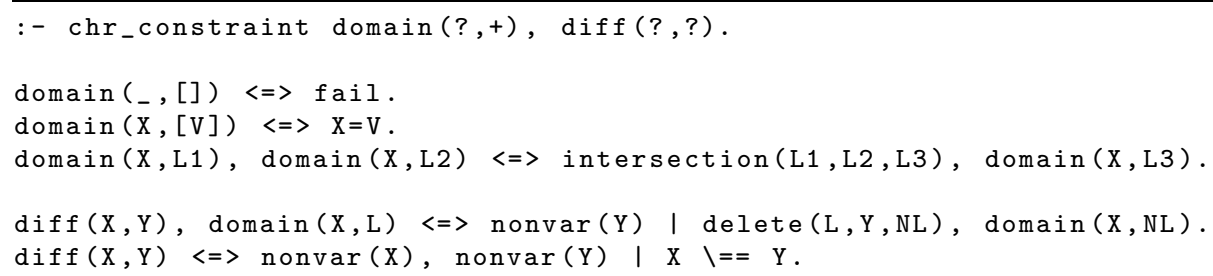

5. The green house is immediately to the right of the ivory house.

6. The Old Gold smoker owns snails.

7. Kools are smoked in the yellow house.

8. Milk is drunk in the middle house.

9. The Norwegian lives in the first house on the left.

10. The man who smokes Chesterfields lives next to the man with the fox.

11. Kools are smoked in the house next to the house where the horse is kept.

12. The Lucky Strike smoker drinks orange juice.

13. The Japanese smokes Parliaments.

14. The Norwegian lives next to the blue house.

The question is: who drinks water, and who owns the zebra?

Example 7 (zebra puzzle). Puzzles like the zebra puzzle can be solved using the CHR program by Pieter Valcke and Tom Schrijvers, shown in Listing 3.5.

The ZEBRA program defines two constraints: domain/2, which can be used to declare the finite domain from which an unknown variable can take a value, and $\operatorname{diff} / 2$, which declares two variables to have a different value.

Using these two elementary constraints, more complicated constraints can be defined, either in CHR or in the host language Prolog. Listing 3.6 shows an encoding of the zebra puzzle. A list of lists S represents a full solution: each sublist represents one house. The auxiary predicate domains $/ 2$ is used to assign the same domain of colors, nationalities, etc. to the relevant variables. The auxiliary predicate all_different/1 takes a list of variables and declares all of them to be different. Most of the clues can be formulated by stating that some partially instantiated sub-list has to be a member of the solution $\mathrm{S}$.

(1) In practice, a better performance can be achieved by stating the "more informative" clues first: for examples clues 8 and 9 should be put first. 
Listing 3.6: Formulation of the zebra puzzle

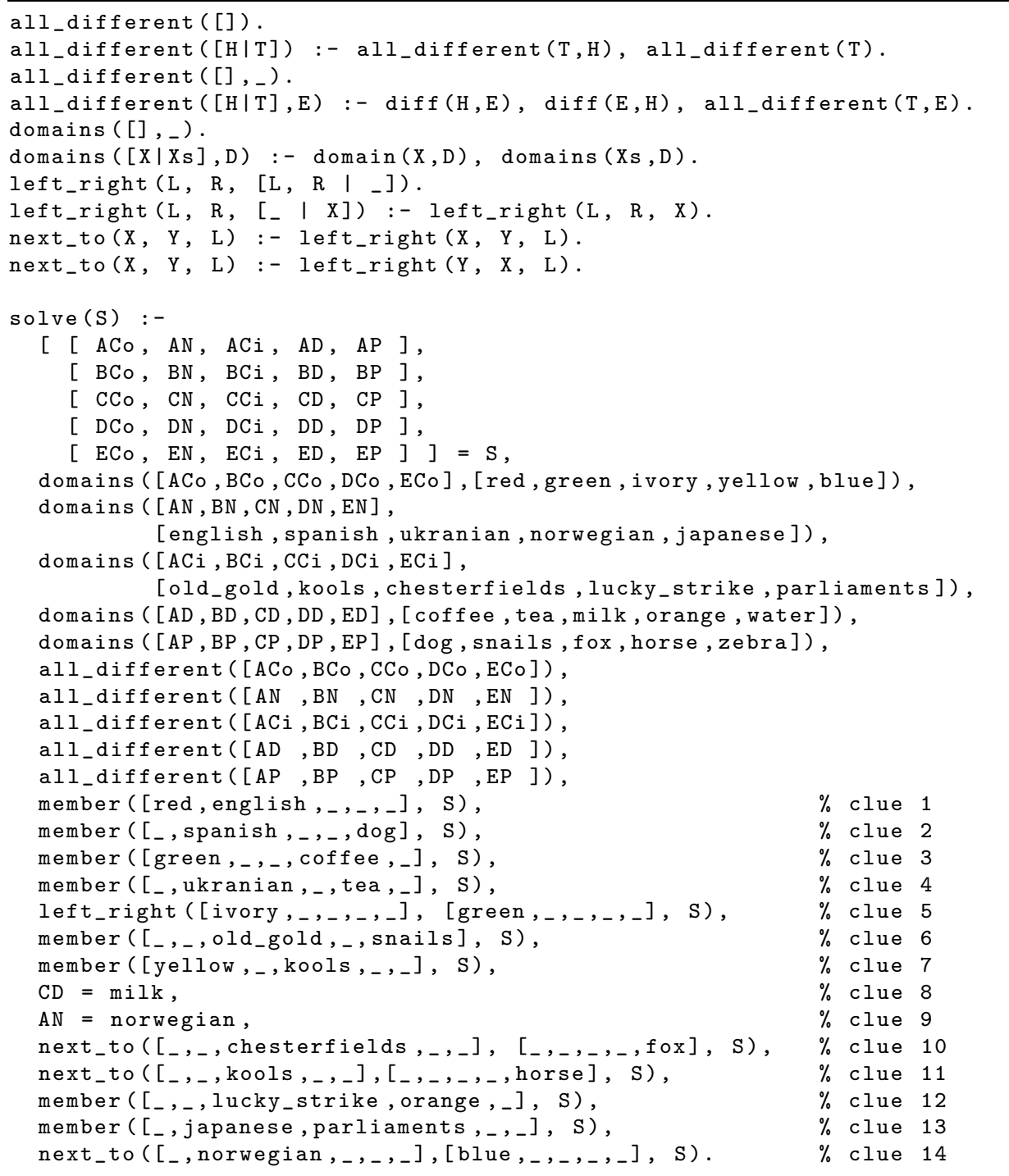


Listing 3.7: SUDOKU: Solver for Sudoku puzzles

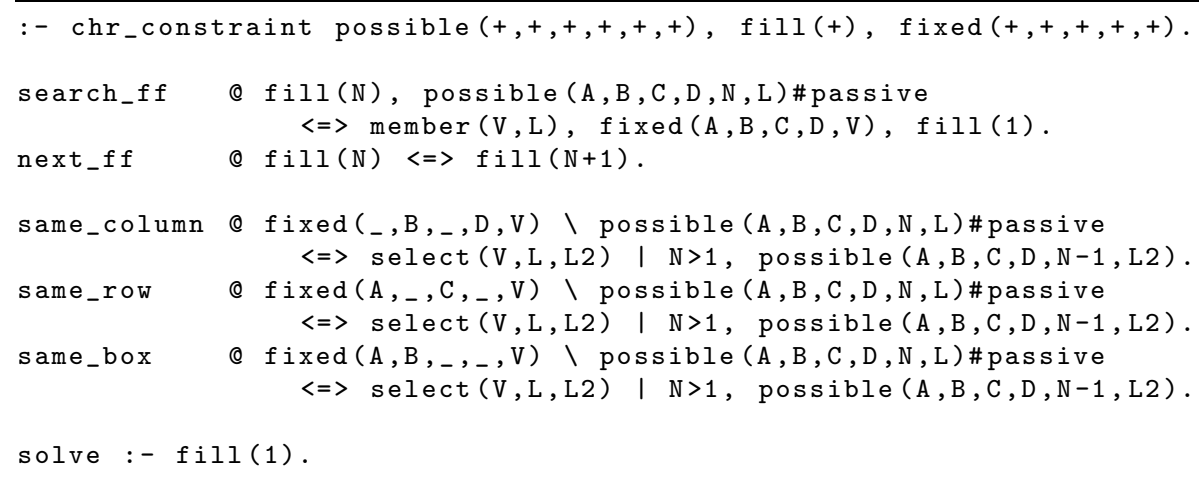

\subsubsection{Sudoku puzzle solver}

Sudoku is a Japanese puzzle which relatively recently attained international popularity. Sudoku is played on a 9x9 board, consisting of 9 smaller $3 \times 3$ boxes. Initially, most board cells are empty and some have a value between 1 and 9 . The aim is to fill the empty cells such that the same number does not occur more than once on every row, column, and box. Normally there is only one valid solution.

Example 8 (Sudoku). Listing 3.7 shows an implementation of a Sudoku puzzle solver based on Thom Frühwirth's rewrite of Jon Murua González' and Henning Christiansen's program.

In the SUDOKU program, every board cell is identified using 4 coordinates: the first two refer to one $3 \times 3$ box, the other two give the position inside that box. The first four arguments of the constraints fixed $/ 5$ and possible $/ 6$ refer to the coordinates of a board cell. The constraint fixed $/ 5$ indicates that the value of a board cell is known; in a query, this constraint is used to input the given cells. If the value of a board cell is not yet known, the constraint possible/ 6 contains the number of remaining possible values and the list of values. For every empty board cell, the query should contain a constraint of the form possible $(\ldots, 9,[1,2,3,4,5,6,7,8,9])$.

The rules same_column, same_row, and same_box are very similar: if a board cell is known, its value is removed from the list of possibilities of any board cell in the same column (row, box); if this was the last possibility ( program fails. These three rules remove all possible values that are directly in violation with the rules of Sudoku. The first two rules implement a search using the first-fail principle, that is, unknown board cell with few remaining possible 


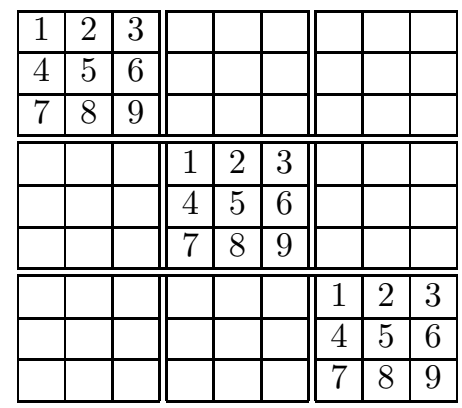

Figure 3.3: Sudoku board layout with 283576 solutions.

values are tried first. The search starts with the fill(1) constraint. If there is a cell which has only one possible value, then the possible/6 constraint is replaced with a fixed $/ 5$ constraint, and then another cell is tried with only one possible value. Note that the new fixed $/ 5$ constraints may cause further applications of the same_* rules. If no more cells with only one possible value are found, then the rule next_ff kicks in, adding fill(2) which looks for a cell with only two possible values, and so on. If there is more than one possible value, the call to member $/ 2$ in the first rule nondeterministically chooses one of the values. If the chosen value turns out to be wrong (i.e. it leads to failure later on), Prolog will backtrack and try a different choice.

(1) The SUDOKU program is in fact a $\mathrm{CHR}^{\vee}$ program (cf. Section 3.4.1) because of the call to the nondeterministic Prolog predicate member $/ 2$. The disjunction is hidden in the definition of member $/ 2$.

In Section 8.4 we will use the SUDOKU program to evaluate a compiler optimization. For the purpose of benchmarking, puzzles with only one solution are not very suitable since they can be solved very quickly. Instead, we will use an underspecified puzzle as a benchmark. The benchmark consists of finding all 283576 solutions for Sudoku boards of the form shown in Figure 3.3.

\subsection{Applications of CHR}

In this section, we give a very brief overview of recent applications of CHR. A more exhaustive overview can be found in (Sneyers, Van Weert, Schrijvers, and De Koninck 2008). 


\subsubsection{Constraint solvers}

CHR was originally designed specifically for writing constraint solvers. Some recent examples are the following:

Lexicographic order. Frühwirth (2006a) presented a constraint solver for a lexicographic order constraint in terms of inequality constraints offered by the underlying solver.

Rational trees. Meister et al. (2006) presented a solver for existentially quantified conjunctions of non-flat equations over rational trees. Djelloul et al. (2007) use this solver for rational trees as a component of a more general solver for (quantified) first-order constraints over finite and infinite trees.

Non-linear constraints. A general purpose CHR-based CLP system for nonlinear (polynomial) constraints over the real numbers was presented by De Koninck et al. (2006a). The system, called $\operatorname{INCLP}(\mathbb{R})$, is based on interval arithmetic and uses an interval Newton method as well as constraint inversion to achieve respectively box and hull consistency.

Solvers derived from union-find. Frühwirth (2006b) has proposed linear-time algorithms for solving certain boolean equations and linear polynomial equations in two variables. These solvers are derived from the classic union-find algorithm (see Section 5.1).

Soft constraints. An important class of constraints are the so-called soft constraints which are used to represent preferences amongst solutions to a problem. Unlike hard (required) constraints which must hold in any solution, soft (preferential) constraints must only be satisfied as far as possible. Bistarelli et al. (2004) have presented a series of constraint solvers for (mostly) extensionally defined finite domain soft constraints.

Another well-known formalism for describing over-constrained systems is that of constraint hierarchies, where constraints with hierarchical strengths or preferences can be specified, and non-trivial error functions can be used to determine the solutions. Wolf (2000b) has proposed an approach for solving dynamically changing constraint hierarchies.

Scheduling. Abdennadher and Marte (2000) have successfully used CHR for scheduling courses at the university of Munich. Their approach is based on soft constraints to deal with teacher's preferences. The related problem of assigning classrooms to courses given a timetable, is dealt with in (Abdennadher, Saft, and Will 2000).

Spatio-temporal reasoning. In the context of autonomous mobile robot navigation, a crucial research topic is automated qualitative reasoning about 
spatio-temporal information, including orientation, named or compared distances, cardinal directions, topology and time. The use of CHR for spatiotemporal reasoning has received considerable research attention. We mention in particular the contributions of Escrig et al. (Escrig and Toledo 1998a, 1998b; Cabedo and Escrig 2003).

Multi-agent systems. FLUX (Thielscher 2002, 2005) is a high-level programming system, implemented in CHR and based on fluent calculus, for cognitive agents that reason logically about actions in the context of incomplete information. An interesting application of this system is FLUXPLAYER (Schiffel and Thielscher 2007), which won the 2006 General Game Playing (GGP) competition at AAAI'06. Seitz et al. (2002) and Alberti et al. (Alberti and Daolio et al. 2004; Alberti and Gavanelli et al. 2004, 2006) have also applied CHR in the context of multi-agent systems. Lam and Sulzmann (2006) have explored the use of CHR as an agent specification language, founded on CHR's linear logic semantics (see Section 3.1.3).

Data integration. One of the core problems related to the so-called Semantic $W e b$ is the integration and combination of data from diverse information sources. Bressan and Goh (1998) have described an implementation of the COIN (Context INterchange) mediator that uses CHR for solving integrity constraints. In more recent work, CHR has been used for implementing an extension of the COIN framework, capable of handling more data source heterogeneity (Firat 2003). Badea et al. (2004) have presented an improved mediator-based integration system.

Description logic. The Web Ontology Language (OWL) is based on Description Logic (DL). Various rule-based formalisms have been considered for combination and integration with OWL or other description logics. Frühwirth (2007) has proposed a CHR-based approach to DL and DL rules.

\subsubsection{Automatic solver generation}

Many authors have investigated the automatic generation of CHR rules from a formal specification. Most works consider extensionally defined constraints over (small) finite domains as the specification.

Apt and Monfroy (2001) have shown how to derive propagation rules from an extensional definition of a finite domain constraint. As an extension, Brand and Monfroy (2003) have proposed to transform the derived rules to obtain stronger propagation rules. Brand (2002) has proposed a method to eliminate redundant propagation rules.

Abdennadher and Rigotti (2004) have also derived propagation rules from extensionally defined constraints. In contrast to the approach of Apt and Monfroy 
(2001), rules are assembled from given parts and propagation rules are transformed into simplification rules if possible. In (Abdennadher and Rigotti 2005) the approach is extended to intensional constraint definitions, where constraints are defined by logic programs. The latter is further extended in (Abdennadher and Sobhi 2008) to symbolically derive rules from the logic programs, rather than from given parts.

\subsubsection{Type systems}

CHR's aptness for symbolic constraint solving has led to many applications in the context of type system design, type checking and type inference. While the basic Hindley-Milner type system requires no more than a simple Herbrand equality constraint, more advanced type systems require custom constraint solvers.

The most successful use of CHR in this area is for Haskell type classes. Type classes are a principled approach to ad hoc function overloading based on typelevel constraints. By defining these type class constraints in terms of a CHR program (Stuckey and Sulzmann 2005) the essential properties of the type checker - soundness, completeness and termination - can be established. Moreover, various extensions, such as multi-parameter type classes (Sulzmann, Schrijvers, and Stuckey 2006) and functional dependencies (Sulzmann, Duck, Peyton-Jones, and Stuckey 2007) are easily expressed.

\subsubsection{Abduction}

Abduction is the inference of a cause to explain a consequence: given $B$ determine $A$ such that $A \rightarrow B$. It has applications in many areas: diagnosis, recognition, natural language processing, type inference, ...

The earliest work connecting CHR with abduction is that of Abdennadher and Christiansen (2000). It shows how to model logic programs with abducibles and integrity constraints in CHR ${ }^{\vee}$. The HYPROLOG system of Christiansen and Dahl (2005) combines abductive reasoning and abductive-based logic programming in one system. Christiansen (2006) has also proposed the use of CHR for the implementation of global abduction, an extended form of logical abduction for reasoning about a dynamic world.

\subsubsection{Computational linguistics}

CHR allows flexible combinations of top-down and bottom-up computation (Abdennadher and Schütz 1998), and abduction fits naturally in CHR as well (see Section 3.6.4). It is therefore not surprising that CHR has proven a powerful implementation and specification tool for language processors.

The most successful approach to CHR-based language processing is that of CHR grammars (CHRG), a highly expressive, bottom-up grammar specification 
language proposed by Christiansen (2005a). Christiansen recognizes that the CHR language itself can be used as a powerful grammar formalism. CHRG's, built as a relatively transparent layer of syntactic sugar over CHR, are to CHR what DCG's are to Prolog.

Applications of CHRG. Using CHRG, Dahl and Blache (2005) have developed directly executable specifications of property grammars. In (Dahl and Gu 2006), an extension of this approach is used to extract concepts and relations from biomedical texts. Dahl and Voll (2004) have generalized the property grammar parsing methodology into a general concept formation system. Applications of this formalism include early lung cancer diagnosis (Barranco-Mendoza 2005, Chapter 4), error detection and correction of radiology reports obtained from speech recognition (Voll 2006, Section 5.2.8), and the analysis of biological sequences (Bavarian and Dahl 2006).

\subsubsection{Testing and verification}

Another application domain for which CHR has proved useful is software testing and verification. Ribeiro et al. (2000) have presented a CHR-based tool for detecting security policy inconsistencies. Lötzbeyer and Pretschner et al. (2000, 2004) have proposed a model-based testing methodology, in which test cases are automatically generated from abstract models using CLP and CHR. They considered the ability to formulate arbitrary test case specifications by means of CHR to be one of the strengths of their approach. Gouraud and Gotlieb (2006) have used a similar approach for the automatic generation of test cases for the Java Card Virtual Machine (JCVM). A formal model of the JCVM is automatically translated into CHR, and the generated CHR program is used to generate test cases.

More of an exploration than testing application is the JMMSOLVE framework (Schrijvers 2004). Its purpose is to explore and test the behavior of declarative memory models for Java, based on the Concurrent Constraint-based Memory Machines proposal of Vijay Saraswat.

\subsection{Related formalisms}

The relation of CHR to other formalisms has recently received quite a lot of attention. In this section we give a brief overview.

As a general remark, we should mention that most of the formalisms related to CHR are limited to ground terms and lack the equivalent of propagation rules. In that sense, they are subsumed by CHR. Also, CHR can be seen as an instance or a generalization of concurrent constraint programming, constraint logic programming, constraint databases, and deductive databases. 
Logical formalisms. In Section 3.1.3, we discussed the logical semantics of CHR. CHR can be given a classical logic semantics (Frühwirth 1998), a linear logic semantics (Betz and Frühwirth 2005, 2007), and a transaction logic semantics (Meister, Djelloul, and Robin 2007). Also, it should be noted that $\mathrm{CHR}^{\vee}$ subsumes Prolog. Sarna-Starosta et al. (2008) have constructed an embedding of a fragment of CHR into an extension of Datalog. Frame-Logic (F-Logic) is an object-oriented extension of classical predicate logic. Kaeser and Meister (2006) have explored the relation between CHR and F-Logic by implementing (a fragment of) F-Logic in CHR. Finally, Aurélio et al. (2008) have investigated the relation between default logic and $\mathrm{CHR}^{\vee}$ with negation as failure.

Term rewriting. CHR can be considered as associative and commutative (AC) term rewriting of flat conjunctions. The term rewriting literature inspired many results for CHR, for example on confluence and termination (see Section 3.2).

Duck et al. (2006) have proposed the formalism of ACD term rewriting (the D stands for distributivity), which subsumes both AC term rewriting and CHR. Raiser and Frühwirth (2008) have studied the problem of embedding term graph rewriting in $\mathrm{CHR}^{\mathrm{rp}}$, a variant ${ }^{15}$ of $\mathrm{CHR}$.

\subsubsection{Join-Calculus}

The join-calculus is a calculus for concurrent programming, with both stand-alone implementations and extensions of general purpose languages, such as JoCaml (OCaml), Join Java and Polyphonic C\#.

Sulzmann and Lam (2007b) propose a Haskell language extension for supporting join-calculus-style concurrent programming, based on CHR. Join-calculus rules, called chords, are essentially guardless simplification rules with linear match patterns. In a linear pattern, different head conjuncts are not allowed to share variables. Hence, CHR offers considerably increased expressivity over the joincalculus: propagation rules, general guards and non-linear patterns.

\subsubsection{Logical Algorithms}

CHR is strongly related to the Logical Algorithms (LA) formalism by Ganzinger and McAllester (2002). LA programs can be translated into CHR $^{\text {rp }}$ programs (De Koninck, Schrijvers, and Demoen 2007a). The reverse translation is only possible for subset of $\mathrm{CHR}^{\mathrm{rp}}$ since the LA language lacks the ability to plug in a built-in constraint theory, and also only supports ground constraints (called assertions in LA terminology). The correspondence between both languages makes it possible to apply the meta-complexity result for LA to a subset of $\mathrm{CHR}^{\mathrm{rp}}$. It is

\footnotetext{
${ }^{15} \mathrm{CHR}^{\mathrm{rp}}$ is a variant of $\mathrm{CHR}$ which allows more execution control by means of rule priorities. We discuss it in Section 4.1.4.
} 
also interesting that the first actual implementation of LA is that of De Koninck et al. (2007a), which compiles the language into (regular) CHR rules.

\subsubsection{Graph Transformation Systems}

Raiser (2007) describes an elegant embedding of Graph Transformation Systems (GTS) in CHR. The confluence properties (see Section 3.2.1) for CHR and GTS are similar; in particular, a sufficient criterion for confluence of a GTS is the confluence of the corresponding CHR program. Using a slightly weaker notion of confluence of CHR (in the spirit of observable confluence; Duck, Stuckey, and Sulzmann 2007), standard CHR confluence checkers can be reused to decide GTS confluence.

\subsubsection{Petri nets}

Petri nets are a well-known formalism for the modeling and analysis of concurrent processes. Betz (2007) provides a first study of the relation between CHR and Petri nets. He provides a sound and complete translation of place/transition nets (Jantzen and Valk 1979) - a standard variant of Petri nets - into a small segment of CHR. Place/transition nets are, unlike CHR, not Turing-complete (cf. Section 10.2). A translation of a significant subsegment of CHR into colored Petri nets is presented as well by Betz (2007). This work is a promising first step towards cross-fertilization between both formalisms. Results from Petri nets could for instance be applied to analyze concurrency properties of CHR programs. 


\title{
Chapter 4
}

\section{Compilation of CHR}

\author{
"I mean, if 10 years from now, when you are doing something \\ quick and dirty, you suddenly visualize that I am looking over your \\ shoulders and say to yourself 'Dijkstra would not have liked this', \\ well, that would be enough immortality for me." \\ — Edsger Dijkstra (1930-2002), EWD 1213, August 1995 \\ "The 'confuse_gcc' goto is used only to get better assembly code.. \\ Dijkstra probably hates me."
}

— Linus Torvalds (1969-), August 1994

(comment in kernel/sched.c of Linux version 1.1.42)

In this chapter we give an overview of some CHR compilation techniques, as explained in more detail in the Ph.D. theses of Duck (2005) and Schrijvers (2005).

Section 4.1 gives a high-level overview of the different compilation schemes. We discuss the standard compilation scheme in more detail in Section 4.2. Section 4.3 deals with the refined operational semantics of CHR, which was introduced as a formalization of the execution flow induced by this compilation scheme. Finally, Section 4.4 covers some optimizations for the standard compilation scheme.

\subsection{Overview}

The first CHR compilation scheme was described by Frühwirth and Brisset (1995), for ECL ${ }^{i} \mathrm{PS}^{e}$ Prolog, a CLP system which was originally developed at the European Computer-Industry Research Centre in Munich. Holzbaur and Frühwirth (1999, 2000a) have adapted this scheme from $\mathrm{ECL}^{i} \mathrm{PS}^{e}$ 's fairly specific suspension mechanism to the more primitive and flexible attributed variables feature found in SICStus Prolog. The latter compilation scheme has been adopted by various 
CHR systems (e.g. HALCHR, the Leuven CHR system). Duck et al. (2004) have constructed a formal operational semantics, called the refined semantics (see Section 4.3), that corresponds to this compilation scheme, which we will call the standard compilation scheme because of its widespread use.

\subsubsection{Standard compilation scheme}

In essence, the standard scheme maps each constraint to a procedure, implemented in the host language. Asserting the constraint then corresponds to calling the procedure. This procedure puts the new constraint in the constraint store data structure, and attempts to fire (apply) rules involving the new constraint. The constraint now becomes active. In order to fire the applicable rules, there is a sub-procedure for each occurrence of the constraint symbol in a rule. The main constraint procedure calls these occurrence sub-procedures in the textual order of the rules.

When all occurrences are tried (and none of the rule applications caused the active constraint to be removed), the constraint is suspended: it remains in the constraint store as a passive constraint. Passive constraints can be triggered (or reactivated) when new built-in constraints arrive, since new rules may become applicable when new built-in constraints cause a previously unsatisfied guard to become satisfied. Reactivation of a constraint is done by calling the constraint procedure again (and hence the occurrence sub-procedures); again, the constraint is active until it is suspended, and so on.

(1) The standard compilation scheme was originally designed for the built-in constraint theory of Prolog: Herbrand equations. Duck et al. (2003) show how it can be extended to cover arbitrary constraint theories and solvers.

Each occurrence sub-procedure looks up the required additional head constraints (in the case of multi-headed rules) in the constraint store data structure. These additional head constraints are called partner constraints. If a combination of partner constraints is found, both the guard and propagation history are checked. If all tests succeed, the rule is committed to: an entry is added to the propagation history, the appropriate matching constraints are removed from the constraint store and the body of the rule is executed.

When a rule body (or an initial goal) is executed, the current procedure is interrupted and the body constraints are activated from left to right. This results in a depth-first execution, similar to the way Prolog programs are executed.

Section 4.2 discusses the standard compilation scheme in more detail, for the host language Prolog. First we briefly mention some alternatives to the standard compilation scheme. 


\subsubsection{Schemes based on the standard compilation scheme}

The compilation schemes used by CCHR (Wuille, Schrijvers, and Demoen 2007) and by the Leuven JCHR system (Van Weert, Schrijvers, and Demoen 2005) are based on the standard compilation scheme, modified to fit an imperative language (Van Weert, Wuille, Schrijvers, and Demoen 2008). The compilation scheme used by the Leuven JCHR system is described in detail in (Van Weert 2008).

(1) An important issue is that Java and C typically cannot handle deep use of recursive calls. To avoid frequent call stack overflows, the standard scheme has to be modified.

DJCHR (Wolf 2001a) also uses a compilation scheme similar to the standard scheme, but it is extended with truth maintenance facilities required for adaptive constraint handling (see Section 3.4.3).

(1) Adaptation is implemented by maintaining justifications. Justifications for constraints and rule applications are implemented efficiently using bit vectors. The runtime system of DJCHR also implements adaptive unification and entailment algorithms. Following the approach of Holzbaur and Frühwirth (1999, 2000a), partner constraints are retrieved using a form of attributed variables (Wolf 2001b).

\subsubsection{Other compilation schemes}

Both JaCK (Schmauß 1999) and CHORD have an approach that differs from that of most other CHR compilers. Their front-end transforms the CHR source files to Java code that initializes the data structures of a generic runtime. CHR programs are then essentially interpreted. Because almost all code is generic both systems have a high runtime overhead that could be avoided by compiling to specialized, lower-level code.

The standard compilation scheme is not suited for concurrent implementations of CHR. Lam and Sulzmann (2007) have used software transactional memories (STM), as supported by the Glasgow Haskell Compiler, to implement CHR in a concurrent way. Sulzmann and Lam (2007a) have also explored the use of Haskell's laziness and concurrency abstractions for implementing the search of partner constraints.

Schrijvers et al. (2006) have experimented with an action rules compilation scheme in BProlog. Replicating the exact operational semantics of the standard scheme turned out to be rather hard.

The CHRd system by Sarna-Starosta and Ramakrishnan (2007) was designed to be suitable for tabled execution, and has a constraint store with set semantics, as opposed to the standard multi-set semantics. 
Listing 4.1: P-DIJKSTRA: Dijkstra's shortest path algorithm in CHR $^{\mathrm{rp}}$

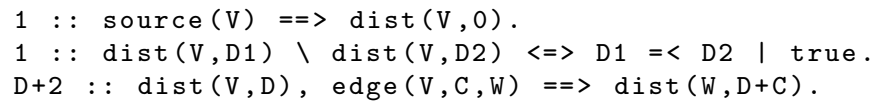

\subsubsection{CHR with rule priorities}

CHR $^{\text {rp }}$ (De Koninck, Schrijvers, and Demoen 2007b) is an extension of CHR with user-definable rule priorities. A rule priority is either a number or an arithmetic expression involving variables that appear in the rule heads. The latter allows different instances of a rule to be executed at different priorities. An example that illustrates the power of dynamic priorities is the $\mathrm{CHR}^{\mathrm{rp}}$ implementation of Dijkstra's algorithm shown in Listing 4.1. The priority of the last rule makes sure that new distance labels are propagated in the correct order: first the nodes closest to the source node (see also Section 5.2).

A compilation scheme for $\mathrm{CHR}^{\mathrm{rp}}$ that is based on the standard scheme, is presented in (De Koninck, Stuckey, and Duck 2008). The main differences are the following. The initial goal, as well as rule bodies, are executed in batch mode (as opposed to depth-first from left to right), i.e., no rule can fire as long as there are unprocessed goal or body constraints. New constraints are scheduled for activation at all priorities at which they have occurrences, instead of being activated as soon as they are processed. Constraints are activated at a given priority and as such only consider those rules that share this priority. Finally, after each rule firing, it is checked whether constraints are scheduled at a higher priority than the rule priority, in which case the highest priority one is activated.

\section{Priority semantics $\omega_{p}$}

The control flow of CHR ${ }^{\mathrm{rp}}$ has been defined formally by De Koninck et al. (2007b) as follows. The $\omega_{p}$ semantics is an instantiation of $\omega_{t}$ (see Section 3.1.4) which ensures that of all applicable rules, the one with the highest priority is applied first. Figure 4.1 shows the transitions of $\omega_{p}$.

\subsection{Implementing the standard scheme}

From now on, we will restrict ourselves to the standard compilation scheme and we will formulate everything in terms of the host language Prolog, unless otherwise mentioned. Most of the compilation techniques that we will discuss can be transferred to other settings in a more or less straightforward way (although sometimes the technical hurdles might be somewhat tedious), but for simplicity we 
1. Solve. $\langle\{c\} \uplus \mathbb{G}, \mathbb{S}, \mathbb{B}, \mathbb{T}\rangle_{n} \succ_{\omega_{p}, \mathcal{P}}\langle\mathbb{G}, \mathbb{S}, c \wedge \mathbb{B}, \mathbb{T}\rangle_{n}$ where $c$ is a built-in constraint.

2. Introduce. $\langle\{c\} \uplus \mathbb{G}, \mathbb{S}, \mathbb{B}, \mathbb{T}\rangle_{n} \mapsto_{\omega_{p}, \mathcal{P}}\langle\mathbb{G},\{c \# n\} \cup \mathbb{S}, \mathbb{B}, \mathbb{T}\rangle_{n+1}$ where $c$ is a CHR constraint.

3. Apply. $\left\langle\emptyset, H_{1} \uplus H_{2} \uplus \mathbb{S}, \mathbb{B}, \mathbb{T}\right\rangle_{n} \longmapsto \omega_{p}, \mathcal{P}\left\langle C, H_{1} \cup \mathbb{S}, \theta \wedge \mathbb{B}, \mathbb{T} \cup\{h\}\right\rangle_{n}$ where $\mathcal{P}$ contains a (renamed apart) rule of priority $p$ of the form $p::$ $r @ H_{1}^{\prime} \backslash H_{2}^{\prime} \Longleftrightarrow G \mid C$ and a matching substitution $\theta$ exists such that $\operatorname{chr}\left(H_{1}\right)=\theta\left(H_{1}^{\prime}\right), \operatorname{chr}\left(H_{2}\right)=\theta\left(H_{2}^{\prime}\right), \mathcal{D} \models \mathbb{B} \rightarrow \bar{\exists}_{\mathbb{B}}(\theta \wedge g), \theta(p)$ is a ground arithmetic expression and $h=\left(r, i d\left(H_{1}\right), i d\left(H_{2}\right)\right) \notin \mathbb{T}$. Furthermore, no rule of priority $p^{\prime}$ and substitution $\theta^{\prime}$ exists with $\theta^{\prime}\left(p^{\prime}\right)<\theta(p)$ for which the above conditions hold.

Figure 4.1: Transitions of the priority semantics $\omega_{p}$

will usually consider only the case of CHR(Prolog) and the standard compilation scheme.

The following code is generated for a constraint $\mathrm{c} / \mathrm{m}$ which has $n$ occurrences in the CHR program:

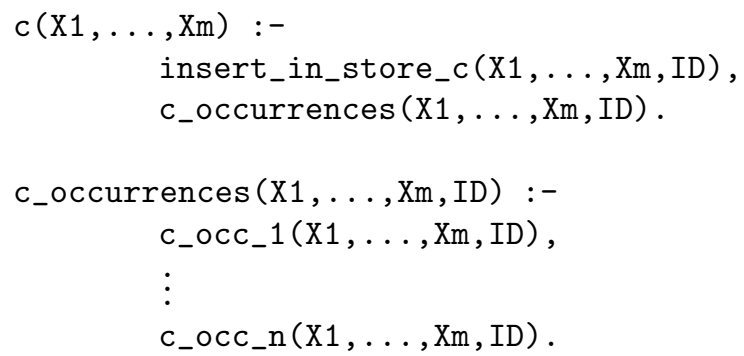

In effect, a Prolog predicate is defined for each CHR constraint, such that CHR constraints can be used freely and transparantly in queries, rule bodies, or even inside other Prolog predicates. The predicate insert_in_store_c $/ m+1$ is an auxiliary predicate that inserts the constraint in some data structure that implements (part of) the constraint store ${ }^{1}$ and assigns to the constraint a unique constraint identifier (ID). The identifier is used to differentiate among multiple copies of syntactically identical constraints; it is also used in the propagation history (cf. Section 3.1.4).

The predicate c_occurrences $/ m+1$ simply calls the $n$ subprocedures for the occurrences of the constraint $\mathrm{c} / \mathrm{m}$. When a constraint needs to be reactivated,

\footnotetext{
${ }^{1}$ The implementation of the constraint store data structures is discussed in Chapter 7 .
} 
this predicate is called - on reactivation, a constraint should not be inserted in the constraint store again.

The subprocedure for an occurrence of $\mathrm{c} / \mathrm{m}$, say the $i$-th occurrence, is constructed as follows. For simplicity we use an overline to indicate an arbitrary number of arguments: instead of $\mathrm{c}(\mathrm{X} 1, \ldots, \mathrm{Xm})$ we write $\mathrm{c}(\overline{\mathrm{X}})$. Assume that the $i$-th occurrence of $\mathrm{c} / \mathrm{m}$ is in some rule $\mathrm{r}$ of the form:

$r @ k_{-} 1(\overline{A 1}), \ldots, k_{-} j(\overline{A j}), c(\bar{X}) \backslash r p_{-} 1(\overline{B 1}), \ldots, r p_{-} k(\overline{B k})$ $\Leftrightarrow$ Guard | Body.

Figure 4.2 shows the code that implements the sub-procedure for this occurrence. The code iterates over all possible partner constraints: the auxiliary predicate universal_lookup_kp_x/1 returns a list of all kp_x constraints; the nested calls to c_occ_i_x gather all the necessary candidate kept partner constraints. Finally in the most deeply nested predicate, c_occ_i_rest, the removed partner constraints are gathered using the auxiliary predicates existential_lookup_rp_*/1, which non-deterministically return one candidate partner constraint. Then it is verified whether all the involved constraints are still alive (i.e. present in the constraint store), whether they are all mutually different, whether the guard succeeds and whether no tuple is already present in the propagation history for this combination of partner constraints. If all the tests succeed, the rule can be applied: a new tuple is added to the propagation history, and the body is executed. Execution proceeds by searching for another combination of candidate partner constraints.

\subsubsection{Constraint representation}

In the constraint store data structures, constraints are represented as a Prolog term. Besides the constraint functor and arguments, the representation contains some meta-information about the constraint. The following representation is used:

suspension (ID , MState, Continuation, MHistory, $\mathrm{C}, \mathrm{X}_{1}, \ldots, \mathrm{X}_{n}$ )

The representation is a term with functor suspension and a number of fields (or arguments). Note that its arity depends on the arity of the constraint it represents. This term representation is called constraint suspension or suspension for short. The meaning of the fields is listed in Table 4.1.

Some of the fields in the term are mutable; this is indicated with the initial capital M in their name. They are implemented using the non-standard Prolog built-in setarg/3 that destructively updates an argument of a term.

It is possible that a constraint is removed while its suspension still occurs in an unprocessed part of a list returned by a universal lookup (for some active constraint higher on the execution stack). To prevent such a removed constraint from being used later on as a partner constraint, the MState field in the suspension is updated to removed when a constraint is removed, and only suspensions with the field set 


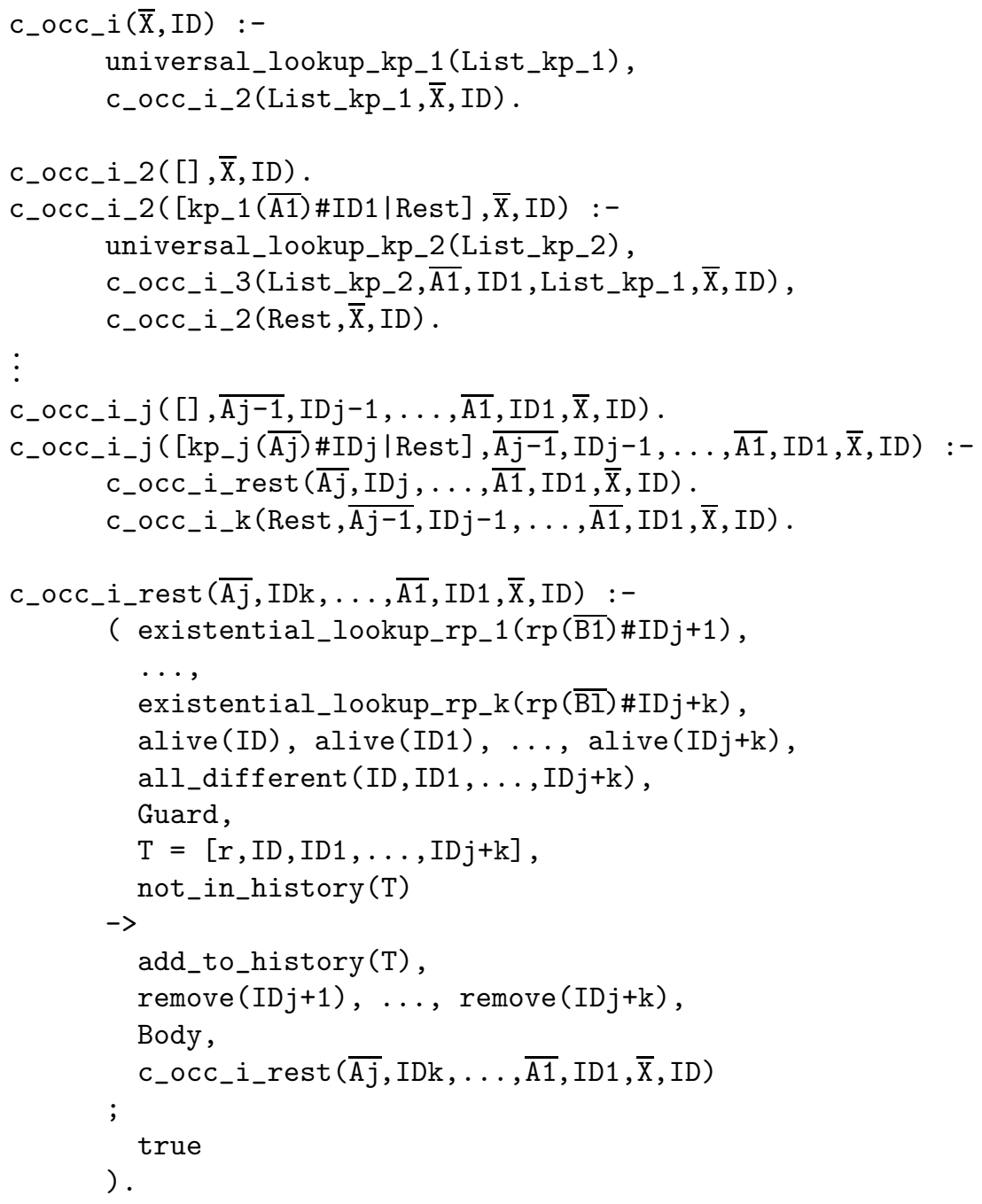

Figure 4.2: Compilation scheme for a constraint occurrence 


\begin{tabular}{c|l}
\hline Field & Meaning \\
\hline ID & $\begin{array}{l}\text { Unique constraint identifier (integer). } \\
\text { State of the suspension. Takes one of three values: } \\
\text { stored - constraint is stored in the constraint store; } \\
\text { removed - constraint is removed from the store; } \\
\text { not_stored - constraint is not (yet) stored (used by } \\
\text { the late storage optimization). }\end{array}$ \\
$\begin{array}{c}\text { Continuation } \\
\text { MHistory }\end{array}$ & $\begin{array}{l}\text { Gart of the distributed propagation history. } \\
\text { Constraint functor. }\end{array}$ \\
$\mathrm{X}_{1}, \ldots, \mathrm{x}_{n}$ & \begin{tabular}{l} 
Arguments of the constraint. \\
\hline
\end{tabular}
\end{tabular}

Table 4.1: Meaning of the constraint suspension fields

to stored are accepted as candidate partner constraints. The auxiliary predicate alive/1 checks this field.

\subsubsection{Some implementation details}

A generic auxiliary predicate insert_constraint_internal is used to create a new suspension term. It uses the Prolog built-in $=. . / 2$ to create the term, initializing the fields as follows:

- a global variable containing current maximal identifier number is incremented and ID is set to its value;

- MState is set to stored (if because of the late storage optimization the constraint is not inserted into the constraint store yet, MState is set to not_stored, or maybe even the creation of the suspension term is delayed);

- The continuation goal Continuation is set to c_occurrences $/ m+1$

- MHistory is set to a data structure representing an empty (partial) history;

\subsection{Refined operational semantics $\omega_{r}$}

As a formal description of the standard compilation scheme (and thus also as a description of the actual behavior of most CHR systems), Duck et al. (2004) have introduced the refined operational semantics $\omega_{r}$ of CHR. It can be shown that $\omega_{r}$ is an instantiation of $\omega_{t}$ - and hence that the standard compilation scheme is correct w.r.t. the $\omega_{t}$ semantics, i.e. it applies only rules that may be applied and execution halts only if there are no more applicable rules. 
(1) Note that, at least in the opinion of the author, the refined operational semantics should be considered primarily as a descriptive operational semantics, and not a normative one. The refined semantics is useful as a formal framework to describe and analyze many existing CHR systems. Although it can be used as a reference semantics, for instance to demonstrate correctness of a compiler optimization in a CHR system that adheres to the refined semantics, it is not essential to the CHR language itself. In general, CHR programmers should rely only on the abstract semantics $\omega_{t}$. It may be tempting, for reasons of execution control, to assume that a program will be executed under the refined operational semantics, and most systems do, but as De Koninck et al. (2007b) rightfully argues, the refined operational semantics does not provide an intuitive mechanism for execution control. In this respect, the priority semantics (see Section 4.1.4) — which is a normative semantics unlike the refined semantics - is much more appropriate. Still, many programs presented in this dissertation (for instance the algorithms of Chapter 5) require execution under the refined semantics. The excuse is that these programs require both execution control and efficient systems in which to execute them, while they were written before the priority semantics was invented.

\subsubsection{Definition of the $\omega_{r}$ semantics}

The refined operational semantics uses a stack of constraints. When a new constraint arrives in the constraint store it is pushed on the stack. The constraint on top of the stack is called the active constraint. The active constraint searches for matching rules, in the order in which this constraint occurs in the program. The constraint is popped from the stack when all occurrences have been tried. When a rule fires, its body is executed immediately, from left to right, suspending the execution of the active constraint while the body is executed. When the constraint becomes topmost again, it resumes its search for matching clauses.

Definition 4.1 (occurrenced identified constraint) An occurrenced identified CHR constraint $c \# i: j$ is an identified constraint $c \# i$ annotated with an occurrence number $j$. This annotation indicates that only matches with occurrence $j$ of constraint $c$ are considered at this point in the execution.

Definition $4.2\left(\omega_{r}\right.$ execution state) An $\omega_{r}$ execution state $\sigma$ is a tuple of the form $\langle\mathbb{A}, \mathbb{S}, \mathbb{B}, \mathbb{T}\rangle_{n}$, where $\mathbb{S}, \mathbb{B}, \mathbb{T}$, and $n$ represent the CHR store, the built-in store, the propagation history and the next free identity number just like before. The execution stack $\mathbb{A}$ is a sequence of constraints, identified CHR constraints and occurrenced identified CHR constraints, with a strict ordering where the top-most constraint is called active.

Execution in $\omega_{r}$ proceeds by exhaustively applying transitions from Figure 4.3 to the initial execution state until the built-in store is unsatisfiable or no transitions are applicable. Initial and final states are defined in the same way as in $\omega_{t}$. 
1. Solve. $\left\langle[c \mid \mathbb{A}], \mathbb{S}^{\prime} \uplus \mathbb{S}, \mathbb{B}, \mathbb{T}\right\rangle_{n} \longmapsto \omega_{r}, \mathcal{P}\left\langle\mathbb{S}+\mathbb{A}, \mathbb{S}^{\prime} \uplus \mathbb{S}, c \wedge \mathbb{B}, \mathbb{T}\right\rangle_{n}$ if $c$ is a built-in constraint and $\mathbb{B}$ fixes the variables of $\mathbb{S}^{\prime}$.

2. Activate. $\langle[c \mid \mathbb{A}], \mathbb{S}, \mathbb{B}, \mathbb{T}\rangle_{n} \longmapsto \omega_{r}, \mathcal{P}\left\langle[c \# n: 1 \mid \mathbb{A}], \mathbb{S}^{\prime}, \mathbb{B}, \mathbb{T}\right\rangle_{n+1}$ if $c$ is a CHR constraint, where $\mathbb{S}^{\prime}=\{c \# n\} \uplus \mathbb{S}$.

3. Reactivate. $\langle[c \# i \mid \mathbb{A}], \mathbb{S}, \mathbb{B}, \mathbb{T}\rangle_{n} \longmapsto_{\omega_{r}}, \mathcal{P}\langle[c \# i: 1 \mid \mathbb{A}], \mathbb{S}, \mathbb{B}, \mathbb{T}\rangle_{n}$

4. Drop. $\langle[c \# i: j \mid \mathbb{A}], \mathbb{S}, \mathbb{B}, \mathbb{T}\rangle_{n} \longmapsto_{\omega_{r}, \mathcal{P}}\langle\mathbb{A}, \mathbb{S}, \mathbb{B}, \mathbb{T}\rangle_{n}$ if there is no $j$-th occurrence of $c$ in $\mathcal{P}$.

5. Simplify. $\left\langle[c \# i: j \mid \mathbb{A}],\{c \# i\} \uplus H_{1} \uplus H_{2} \uplus H_{3} \uplus \mathbb{S}, \mathbb{B}, \mathbb{T}\right\rangle_{n}$ $\succ_{r}, \mathcal{P}\left\langle C++\mathbb{A}, H_{1} \uplus \mathbb{S}, \theta \wedge \mathbb{B}, \mathbb{T} \cup\{h\}\right\rangle_{n}$ if the $j$-th occurrence of the constraint $c$ is $d_{j}$ in a rule $r$ in $\mathcal{P}$ of the form $r @ H_{1}^{\prime} \backslash H_{2}^{\prime}, d_{j}, H_{3}^{\prime} \Longleftrightarrow G \mid C$, a matching substitution $\theta$ exists such that $c=\theta\left(d_{j}\right), \operatorname{chr}\left(H_{k}\right)=\theta\left(H_{k}^{\prime}\right)$ for $k=1,2,3, \mathcal{D} \models \mathbb{B} \rightarrow \bar{\exists}_{\mathbb{B}}(\theta \wedge G)$, and $\mathbb{T} \not \supset h=\left(r, i d\left(H_{1}\right), i d\left(H_{2}+c \# i++H_{3}\right)\right)$.

6. Propagate. $\left\langle[c \# i: j \mid \mathbb{A}],\{c \# i\} \uplus H_{1} \uplus H_{2} \uplus H_{3} \uplus \mathbb{S}, \mathbb{B}, \mathbb{T}\right\rangle_{n}$ $\succ_{r}, \mathcal{P}\left\langle C++[c \# i: j \mid \mathbb{A}],\{c \# i\} \uplus H_{1} \uplus H_{2} \uplus \mathbb{S}, \theta \wedge \mathbb{B}, \mathbb{T} \cup\{h\}\right\rangle_{n}$ if the $j$-th occurrence of the constraint $c$ is $d_{j}$ in a rule $r$ in $\mathcal{P}$ of the form $r @ H_{1}^{\prime}, d_{j}, H_{2}^{\prime} \backslash H_{3}^{\prime} \Longleftrightarrow G \mid C$ a matching substitution $\theta$ exists such that $c=\theta\left(d_{j}\right), \operatorname{chr}\left(H_{k}\right)=\theta\left(H_{k}^{\prime}\right)$ for $k=1,2,3, \mathcal{D}=\mathbb{B} \rightarrow \bar{\exists}_{\mathbb{B}}(\theta \wedge G)$, and $\mathbb{T} \not \supset h=\left(r, i d\left(H_{1}+c \# i+H_{2}\right), i d\left(H_{3}\right)\right)$.

7. Default. $\langle[c \# i: j \mid \mathbb{A}], \mathbb{S}, \mathbb{B}, \mathbb{T}\rangle_{n} \longmapsto_{\omega_{r}, \mathcal{P}}\langle[c \# i:(j+1) \mid \mathbb{A}], \mathbb{S}, \mathbb{B}, \mathbb{T}\rangle_{n}$ if no other transition applies.

Figure 4.3: Transitions of the refined operational semantics $\omega_{r}$

\subsubsection{Passive occurrences}

For a given CHR program, it may be the case that some constraint occurrences never actually cause a rule application. Such occurrences are called passive occurrences (not to be confused with a passive constraint, which is a constraint that is not currently active). Passive occurrences can be skipped without even trying to find matching partner constraints. This may result in a significant improvement of the run-time performance. In terms of the $\omega_{r}$ semantics, a passive occurrence is one for which the Default transition can be immediately applied, since the Simplify or Propagate transitions are never applicable for that occurrence.

Some compiler optimizations - e.g. never-stored analysis (Section 4.4.3), but also some of the optimizations in Part II of this dissertation - attempt to automatically identify passive occurrences. Occurrences can also be declared to be passive by the programmer using the annotation \#passive (or the abbreviated 
form \#p). This is useful if the automatic analysis is not sufficiently strong, or if the occurrence is only passive in those derivations that correspond to the intended use of the program, but not in general. Of course, the programmer has to make sure that the passive pragma is only used when it is allowed. Somewhat in the spirit of the green and red Prolog cuts (!/0), we can distinguish between the following:

- A green pragma passive is a use of the \#passive annotation on an occurrence that is in fact passive (in general or in all derivations that correspond to the intended use of the program), i.e. when that occurrence is tried, no matching combination of partner constraints can be found for which the guard holds and for which the rule has not fired before;

- A yellow pragma passive corresponds to an occurrence that is not passive in the above sense, but the \#passive annotation does not have an impact on the derivations according to some observability criterion, i.e. the occurrence may cause a rule to fire which is not essential to the computation (the result would be the same if it did not fire);

- A red pragma passive is a use of the \#passive annotation that does alter derivations in a significant way, i.e. the program no longer functions correctly without the annotation.

We refer to the work of Christiansen (2005b) for a more extensive and formal study of passive occurrences in CHR. In the terminology of Christiansen (2005b), green passive declarations are called control-safe, while yellow passive declarations are called answer-safe (but not control-safe). Red passive declarations are neither control-safe nor answer-safe.

Example 9 (green pragma passive). Consider again the LEQ program of Example 1 (page 30 ). In the second rule, the second occurrence of leq/ 2 can be made passive:

antisymmetry @ leq $(X, Y)$, leq $(Y, X) \#$ passive $\Leftrightarrow X=Y$.

Indeed, we can show that this occurrence never leads to a rule application because a matching partner constraint will never be found, for suppose there would be a matching partner constraint, then this constraint would also have been there when the first occurrence of that rule was tried. But then the rule would have fired already, which would have removed both constraints, contradicting our assumption.

Compiler optimizations can typically only identify green pragmas passive. 
Example 10 (yellow pragma passive). Consider the following program, which computes the symmetric transitive closure of the relation edge/2:

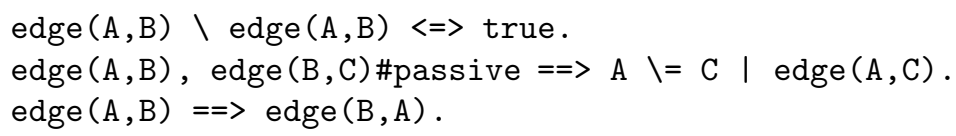

The occurrence with the \#passive annotation can actually cause the rule to fire: for example in the query edge $(a, b)$, edge $(b, c)$, the rule fires when edge $(b, c)$ reaches that occurrence, producing edge $(\mathrm{a}, \mathrm{c})$. However, when later on the last rule produces edge $(c, b)$, the second rule fires again for edge $(c, b)$, edge $(b, a)$ (the latter was created when processing the constraint edge $(a, b)$ from the query), producing edge $(c, a)$, which in turn results in edge $(a, c)$ because of the last rule. So there is another way in which the constraint edge $(a, c)$ can be produced. Since the first rule removes duplicates, the end result is the same regardless of whether or not the indicated occurrence is made passive.

Red pragma passives (and also some of the more exotic kinds of yellow pragma passives) should be avoided by all but the most experienced CHR programmers, because of two reasons: 1) the analyses used in some compiler optimizations may assume that all pragmas can be safely ignored; if this assumption does not hold, the optimization may not be guaranteed to be correct; and 2) it can be very challenging to find the meaning of rules that contain red pragma passives. Still, there are cases in which a red pragma passive can be useful, for instance because it simplifies the program code or allows a more efficient execution than what would be possible without it.

Example 11 (red pragma passive). Assume a number of counter $/ 2$ constraints represent some counters, where the first argument is some identifier and the second argument is the current value of the counter. One way to implement an operation inc_all to increment every counter is as follows:

inc_all \counter $(\mathrm{C}, \mathrm{V}) \#$ passive $\Leftrightarrow$ counter $(\mathrm{C}, \mathrm{V}+1)$.

inc_all $\Leftrightarrow$ true.

Without the \#passive annotation, this program is non-terminating: the first counter that is modified will be incremented ad infinitum. It is not so easy to get the correct behavior without the pragma passive. One solution, which arguably is somewhat less elegant, is as follows:

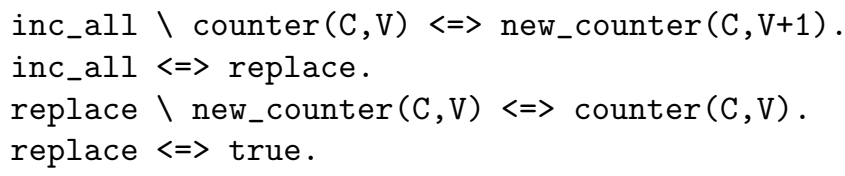




\subsubsection{Call-based refined operational semantics $\omega_{c}$}

The call-based refined operational semantics $\omega_{c}$ is equivalent to the refined operational semantics of CHR. It was formulated by Schrijvers et al. (2005).

Definition $4.3\left(\omega_{c}\right.$ execution state) An $\omega_{c}$ execution state $\sigma$ is a tuple of the form $\langle\mathbb{G}, \mathbb{A}, \mathbb{S}, \mathbb{B}, \mathbb{T}\rangle_{n}$ where $\mathbb{G}, \mathbb{A}, \mathbb{S}, \mathbb{B}, \mathbb{T}$, and $n$ represent (respectively) the goal, call stack, CHR store, built-in store, propagation history, and next free identity number. The goal $\mathbb{G}$ is a sequence of $C H R$ constraints and built-in constraints. The call stack $\mathbb{A}$ is a sequence of occurrenced identified CHR constraints. The other parts of the tuple are as in the $\omega_{r}$ semantics.

Given an initial goal $\mathbb{G}$, the initial state is $\langle\mathbb{G}, \square, \emptyset, \emptyset, \emptyset\rangle_{1}$. As usual, execution proceeds by exhaustively applying transitions to the initial execution state until the built-in solver state is unsatisfiable or no transitions are applicable. The transitions are listed in Figure 4.4.

\subsection{Optimizations}

The basic compilation scheme can be improved in many ways. Over the years, a number of compiler optimizations have been proposed to increase the performance of CHR systems. This section briefly discusses some of these optimizations. For an in-depth treatment of optimizing compilation, we refer to (Holzbaur, García de la Banda, Jeffery, and Stuckey 2001), (Holzbaur, García de la Banda, Stuckey, and Duck 2005), and the Ph.D. dissertations of Duck (2005) and Schrijvers (2005).

In this section only optimizations that were proposed by other authors are covered. Part II of this dissertation will deal with the optimizations to which the author contributed.

\subsubsection{Propagation history maintenance}

For rules in which some head constraints are removed, it is clear that adding a propagation history tuple is redundant: the rule cannot be applied to the same combination of head constraints twice since the first rule application removes some of those heads. Hence the propagation history does not need to be updated for simplification and simpagation rules - this is of course the reason why it is called the propagation history.

The data structure representing the propagation history is often implemented in a distributed way: each constraint keeps track of the history tuples that originated when the rule fired with that constraint as the active constraint. When checking whether a rule has already fired for some combination of constraints, the partial histories have to be checked of both the active constraint and all the candidate partner constraints. The advantage of a distributed propagation history 
1. Solve. $\langle c, \mathbb{A}, \mathbb{S}, \mathbb{B}, \mathbb{T}\rangle_{n} \hookrightarrow_{\omega_{c}, \mathcal{P}}\left\langle\square, \mathbb{A}, \mathbb{S}^{\prime}, \mathbb{B}^{\prime}, \mathbb{T}^{\prime}\right\rangle_{n^{\prime}}$ where $c$ is a built-in constraint. If $\mathcal{D}=\neg \bar{\exists}_{\emptyset}(c \wedge \mathbb{B})$, then $\left(\mathbb{S}^{\prime}, \mathbb{B}^{\prime}, \mathbb{T}^{\prime}, n^{\prime}\right)=(\mathbb{S}, c \wedge \mathbb{B}, \mathbb{T}, n)$. Otherwise choose $\mathbb{S}^{\prime}, \mathbb{B}^{\prime}, \mathbb{T}^{\prime}$, and $n^{\prime}$ such that $\left\langle\mathbb{S}_{1}, \mathbb{A}, \mathbb{S}, c \wedge \mathbb{B}, \mathbb{T}\right\rangle_{n} \succ_{\omega_{c}, \mathcal{P}}^{*}\left\langle\square, \mathbb{A}, \mathbb{S}^{\prime}, \mathbb{B}^{\prime}, \mathbb{T}^{\prime}\right\rangle_{n^{\prime}}$ and $\mathbb{S}_{1}=$ wakeup_policy $(\mathbb{S}, \mathbb{B}, c)$ is a subset of $\mathbb{S}$. The definition of the wakeup policy depends on the host language and the specifics of the implementation.

2a. Activate. $\langle c, \mathbb{A}, \mathbb{S}, \mathbb{B}, \mathbb{T}\rangle_{n} \mapsto_{\omega_{c}, \mathcal{P}}\langle c \# n: 1, \mathbb{A},\{c \# n\} \uplus \mathbb{S}, \mathbb{B}, \mathbb{T}\rangle_{n+1}$ where $c$ is a CHR constraint which has never been active.

2b. Reactivate. $\langle c \# i, \mathbb{A}, \mathbb{S}, \mathbb{B}, \mathbb{T}\rangle_{n} \longmapsto_{\omega_{c}, \mathcal{P}}\langle c \# i: 1, \mathbb{A}, \mathbb{S}, \mathbb{B}, \mathbb{T}\rangle_{n}$ where $c \# i$ is a CHR constraint in the store (back in the queue through Solve).

3. Drop. $\langle c \# i: j, \mathbb{A}, \mathbb{S}, \mathbb{B}, \mathbb{T}\rangle_{n} \longmapsto \omega_{c}, \mathcal{P}\langle\square, \mathbb{A}, \mathbb{S}, \mathbb{B}, \mathbb{T}\rangle_{n}$ where $c \# i: j$ is an occurrenced active constraint and there is no such occurrence $j$ in $\mathcal{P}$.

4. Simplify. $\left\langle c \# i: j, \mathbb{A},\{c \# i\} \uplus H_{1} \uplus H_{2} \uplus H_{3} \uplus \mathbb{S}, \mathbb{B}, \mathbb{T}\right\rangle_{n} \hookrightarrow_{\omega_{c}, \mathcal{P}}\left\langle\square, \mathbb{A}, \mathbb{S}^{\prime}, \mathbb{B}^{\prime}, \mathbb{T}^{\prime \prime}\right\rangle_{n^{\prime}}$ where $\left\langle\theta(C), \mathbb{A}, H_{1} \uplus S, \theta \wedge \mathbb{B}, \mathbb{T}^{\prime}\right\rangle_{n} \longmapsto_{\omega_{c}, \mathcal{P}}^{*}\left\langle\square, \mathbb{A}, \mathbb{S}^{\prime}, \mathbb{B}^{\prime}, \mathbb{T}^{\prime \prime}\right\rangle_{n^{\prime}}$ where the $j$-th occurrence of CHR constraint $c$ is $d_{j}$ in rule $r \in \mathcal{P}$ of the form $H_{1}^{\prime} \backslash H_{2}^{\prime}, d_{j}, H_{3}^{\prime} \Longleftrightarrow G \mid C$ and there exists a matching substitution $\theta$ such that $c=\theta\left(d_{j}\right)$, $\operatorname{chr} H_{x}=\theta\left(H_{x}^{\prime}\right)$ for $x \in\{1,2,3\}$, and $\mathcal{D} \models \mathbb{B} \rightarrow \bar{\exists}_{\text {vars }(r)}(\theta \wedge$ $G)$, and the tuple $h=\left(r, i d\left(H_{1}\right), i d\left(H_{2}\right)++[i]+i d\left(H_{3}\right)\right) \notin \mathbb{T}$. The substitution $\theta$ must also rename apart all variables appearing only in $G$ and $C$. In the intermediate transition sequence $\mathbb{T}^{\prime}=\mathbb{T} \cup\{h\}$. If no such matching substitution exists then $\langle c \# i: j, \mathbb{A}, \mathbb{S}, \mathbb{B}, \mathbb{T}\rangle_{n} \longmapsto_{\omega_{c}, \mathcal{P}}\langle c \# i: j+1, \mathbb{A}, \mathbb{S}, \mathbb{B}, \mathbb{T}\rangle_{n}$

5. Propagate. $\left\langle c \# i: j, \mathbb{A},\{c \# i\} \uplus \mathbb{S}, \mathbb{B}_{0}, \mathbb{T}_{0}\right\rangle_{n_{0}} \mapsto \omega_{c}, \mathcal{P}\left\langle\mathbb{G}, \mathbb{A}, \mathbb{S}_{k}, \mathbb{B}_{k}, \mathbb{T}_{k}\right\rangle_{n_{k}}$ where the $j$-th occurrence of $c$ is $d_{j}$ in rule $r @ H_{1}^{\prime}, d_{j}, H_{2}^{\prime} \backslash H_{3}^{\prime} \Longleftrightarrow G \mid C$.

Let $\mathbb{S}_{0}=\mathbb{S} \uplus\{c \# i\}$. Now assume, for $1 \leq l \leq k$ and $k \geq 0$, we have:

$$
\begin{aligned}
\left\langle C_{l},[c \# i: j \mid \mathbb{A}], H_{1 l} \uplus\{c \# i\} \uplus H_{2 l} \uplus R_{l}, \mathbb{B}_{l-1}, \mathbb{T}_{l-1} \cup\left\{t_{l}\right\}\right\rangle_{n_{l-1}} & \longmapsto_{\omega_{c}, \mathcal{P}}^{*}\left\langle\square,[c \# i: j \mid \mathbb{A}], \mathbb{S}_{l}, \mathbb{B}_{l}, \mathbb{T}_{l}\right\rangle_{n_{l}}
\end{aligned}
$$

where $\{c \# i\} \uplus H_{1 l} \uplus H_{2 l} \uplus H_{3 l} \uplus R_{l}=\mathbb{S}_{l-1}$ and there exists a matching substitution $\theta_{l}$ such that $c=\theta_{l}\left(d_{j}\right), C_{l}=\theta_{l}(C), \operatorname{chr} H_{x l}=\theta_{l}\left(H_{x}^{\prime}\right)$ for $x \in$ $\{1,2,3\}, \mathcal{D} \models \mathbb{B}_{l-1} \rightarrow \bar{\exists}_{\operatorname{vars}\left(\theta_{l}(r)\right)} \theta_{l}(G)$, and $t_{l}=\left(r, i d\left(H_{1 l}\right)+[i]++\right.$ $\left.i d\left(H_{2 l}\right), i d\left(H_{3 l}\right)\right) \notin \mathbb{T}_{l-1}$ where $\theta_{l}$ renames apart all variables only appearing in $G$ and $C$ (separately for each $l$ ). Furthermore, for $k+1$ no such transition is possible. The resulting goal $\mathbb{G}$ is either $\mathbb{G}=\square$ if $\mathcal{D}=\neg \bar{\exists}_{\emptyset} \mathbb{B}_{k}$ (i.e. failure occurred) or $\mathbb{G}=c \# i: j+1$ otherwise.

6. Goal. $\langle[c \mid C], \mathbb{A}, \mathbb{S}, \mathbb{B}, \mathbb{T}\rangle_{n} \mapsto_{\omega_{c}, \mathcal{P}}\left\langle\mathbb{G}, \mathbb{A}, \mathbb{S}^{\prime}, \mathbb{B}^{\prime}, \mathbb{T}^{\prime}\right\rangle_{n^{\prime}}$ where $[c \mid C]$ is a sequence of built-in and CHR constraints and $\langle c, \mathbb{A}, \mathbb{S}, \mathbb{B}, \mathbb{T}\rangle_{n} \longmapsto_{\omega_{c}, \mathcal{P}}^{*}\left\langle\square, \mathbb{A}, \mathbb{S}^{\prime}, \mathbb{B}^{\prime}, \mathbb{T}^{\prime}\right\rangle_{n^{\prime}}$ and $\mathbb{G}=\square$ if $\mathcal{D} \models \bar{\exists}_{\emptyset}\left(\neg \mathbb{B}^{\prime}\right)$ (i.e. calling $c$ caused failure) or $\mathbb{G}=C$ otherwise.

Figure 4.4: Transitions of the call-based refined operational semantics $\omega_{c}$ 
is twofold: firstly, each partial history is (much) smaller than the whole history, so the check and update operations take less time; secondly, when a constraint is removed, a part of the propagation history may be garbage collected by the underlying host language, allowing less space overhead.

In some cases, maintaining a propagation history can be avoided even for propagation rules. For instance, if in a single-headed propagation rule the head constraint is known to have only ground arguments, the propagation history is not needed (the head constraint will not be reactivated).

More advanced optimizations related to the propagation history were recently proposed by Van Weert (2008).

\subsubsection{Late storage}

In the basic compilation scheme, and also in the refined operational semantics, CHR constraints are immediately inserted into the constraint store, even before the first occurrence is tried. In general, this is necessary for correctness because when the active constraint causes a rule to fire, it may itself be needed to be accessible as a partner constraint during the execution of the rule body.

However, in many cases, a CHR constraint has a very short lifetime: it is often removed from the constraint store almost immediately after it has been activated. This leads to unnecessary insertion and removal operations, which could be relatively costly. Therefore, Holzbaur et al. (2001) have proposed an optimization called late storage, which aims at deferring constraint store insertion as long as possible. If insertion can be deferred until a point where the constraint is removed, the overhead of the constraint store operations can be avoided.

Constraint storage is done in two phases: firstly, a suspension term (see Section 4.2.1) is created (this is called constraint allocation), and secondly, the representation is inserted into the data structure(s) representing the constraint store (this is called constraint insertion). In the approach of Holzbaur et al. (2001), constraint allocation is done only just before the first kept occurrence. Constraint insertion is attempted just before the execution of the rule body for all kept occurrences, and just after the last occurrence (in case none of those rules fired). The insertion attempts are implemented in a way that only does the actual insert operation on the first attempt; further insertion attempts are ignored. The suspension field MState (see Table 4.1 on page 64 ) is used to distinguish between a not yet inserted constraint and one that has already been inserted.

Schrijvers et al. (2005) have extended and formalized the approach of Holzbaur et al. (2001) in a framework based on abstract interpretation (see Section 4.4.5). This results in a more accurate late storage analysis. A detailed exposition of the improved approach can be found in chapter 7 of (Schrijvers 2005). 


\subsubsection{Never-stored constraints}

Late storage analysis may reveal that constraint storage is not needed for any of the constraint occurrences. If furthermore the program contains an unconditional single-headed rule that removes the constraint - in other words, a rule of the form $c(\bar{X}) \Leftrightarrow \ldots$ - then we can infer that this constraint will never be stored at all (Holzbaur, García de la Banda, Stuckey, and Duck 2005). Now consider a rule $r$ that contains a never-stored constraint in its head. If one of the other head constraints becomes active and its occurrence in rule $r$ is tried, it will search for a matching combination of partner constraints. The search is doomed to fail since the look-up for the never-stored partner constraint will of course never return any results. This implies that all other head constraints in $r$ can safely be declared to be passive (see Section 4.3.2). If a rule contains two or more never-stored constraints, all heads become passive - in other words, the rule never fires so it can be ignored.

\subsubsection{Functional dependencies}

Given a CHR store $\mathbb{S}$ and a CHR constraint predicate $p\left(a_{1}, \ldots, a_{n}\right)$ of arity $n$, we say there is a functional dependency from the argument positions $K \subseteq\left\{a_{1}, \ldots, a_{n}\right\}$ to the arguments $R \subseteq\left\{a_{1}, \ldots, a_{n}\right\}$ if, given values for the arguments of the key $K$, the arguments of $R$ are uniquely determined for every constraint in $\mathbb{S}$. This is denoted as follows: $p\left(a_{1}, \ldots, a_{n}\right):: K \rightsquigarrow R$. A formal definition and a static analysis to detect functional dependencies was given by Duck and Schrijvers (2005). Their approach is again based on abstract interpretation (see Section 4.4.5). Functional dependency information can be used amongst others to improve indexing data structures and as important input for join ordering heuristics (see Chapter 9).

Example 12 (functional dependencies). In the following program:

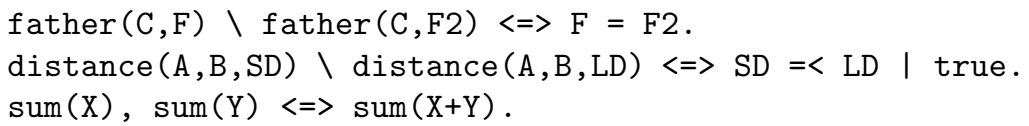

the following functional dependencies can be shown to hold:

$$
\begin{gathered}
\operatorname{father}(C, F)::\{C\} \rightsquigarrow\{F\} \\
\operatorname{distance}(A, B, D)::\{A, B\} \rightsquigarrow\{D\} \\
\operatorname{sum}(X):: \emptyset \rightsquigarrow\{X\}
\end{gathered}
$$

If a constraint (like sum/ 1 in the above example) has a functional dependency of the form $\emptyset \rightsquigarrow A$, where $A$ are all its arguments, the constraint is said to be a singleton constraint. 


\subsubsection{Other optimizations based on abstract interpretation}

Schrijvers et al. (2005) present a general and systematic framework for program analysis of CHR based on abstract interpretation. In sections 4.4 .2 and 4.4.4 we already discussed two analyses that are based on this framework. Another instance of the abstract interpretation framework is groundness analysis. If for a given CHR constraint it can be derived that its arguments are always ground throughout the computation, more efficient indexing data structures can be used. Furthermore, such constraints cannot be triggered, so a lot of overhead related to constraint reactivation (e.g. propagation history maintenance) can be avoided.

The abstract interpretation framework of Schrijvers et al. (2005) can also be used for observation analysis. We say a constraint $c$ observes another constraint $d$ if the presence or absence of $d$ has an influence on the subcomputation caused by activating or reactivating $c$. Note that an observed constraint $d$ does not have to be a partner constraint of $c$ : it could for instance also be a partner constraint of a constraint that is added or triggered by executing the body of a rule involving $c$.

A detailed exposition of the abstract interpretation framework of Schrijvers et al. (2005) can also be found in (Schrijvers 2005).

\subsubsection{Indexing and join ordering}

The time complexity of executing a CHR program often crucially depends on the join ordering - the order in which partner constraints are looked up in order to find matching rules - and the efficiency of the data structures used for partner constraint lookups. In Chapters 7 and 9 we will discuss these important aspects of optimizing CHR compilation. 


\title{
Chapter 5
}

\section{Implementing Classic Algorithms in CHR}

\begin{abstract}
"How do we convince people that in programming simplicity and clarity - in short: what mathematicians call 'elegance' — are not a dispensable luxury, but a crucial matter that decides between success and failure?"
\end{abstract}

— Edsger Dijkstra (1930-2002)

"An algorithm must be seen to be believed."

- Donald Knuth (1938-)

As the name suggests, Constraint Handling Rules was originally designed as a special-purpose language for implementing constraint solvers. Recently, CHR is increasingly being used as a general-purpose programming language. Starting a trend of investigating this side of CHR, Schrijvers and Frühwirth (2005a, 2006) have implemented and analyzed the classic union-find algorithm of Tarjan and van Leeuwen (1984) in CHR. In particular, they showed how the optimal complexity of this algorithm can be achieved in CHR - a non-trivial achievement since this is believed to be impossible in pure Prolog. This work later lead to parallel versions of the union-find algorithm (Frühwirth 2005b) and several derived algorithms (Frühwirth 2006b).

In fact, the work presented in Part III of this dissertation - a general complexitywise completeness result for CHR - was largely inspired by the specific optimal complexity result for the union-find algorithm. Despite the general complexitywise completeness result that we will prove in Chapter 11, the question of finding elegant and natural implementations of classic algorithms in CHR still remains an interesting research topic. 
Listing 5.1: N-UNION-FIND: Naive implementation of the union-find algorithm

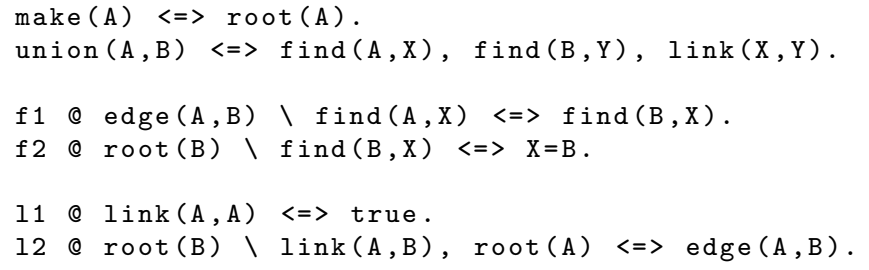

In this chapter, we hope to convince the reader that CHR can be used as 'executable pseudo-code' for studying and designing algorithms while constructing a real, executable program with the desired time (and space) complexity. We discuss three classic algorithms and their implementation in CHR. Firstly, Section 5.1 briefly recaptures the union-find algorithm as implemented by Schrijvers and Frühwirth (2006). Secondly, in Section 5.2, we discuss in considerable detail an implementation of Dijkstra's shortest path algorithm using Fibonacci heaps. Thirdly we construct an implementation of Hopcroft's algorithm for minimizing finite automata in Section 5.3.

\subsection{The union-find algorithm}

A disjoint-set data structure can be used to efficiently maintain an equivalence relation - for example, variable unifications in Prolog. An equivalence relation on some set corresponds to a partition of that set into equivalence classes. A disjoint-set data structure should provide (at least) the following three operations:

- make(X) : add a new element $\mathrm{X}$ (in a new singleton equivalence class);

- union $(\mathrm{X}, \mathrm{Y})$ : assert that elements $\mathrm{X}$ and $\mathrm{Y}$ are equivalent. Their equivalence classes, if different, should be merged;

- $f$ ind $(X, R)$ : find a representative $R$ of the equivalence class of $X$. All members of an equivalence class should have the same representative, so this operation can be used to determine whether two elements are equivalent.

The union-find algorithm of Tarjan and van Leeuwen (1984) can be shown to implement this data structure with optimal time complexity. Essentially, the disjoints sets are represented as trees, where the root node is the representative. The union/ 2 operation adds an edge between the root of one tree and the root of the other tree. The find/2 operation follows the edges until the root is found.

A naive implementation of the union-find algorithm is given in Listing 5.1. The naive version of the union-find algorithm still takes logarithmic time per 
Listing 5.2: UNION-FIND: Optimal implementation of the union-find algorithm

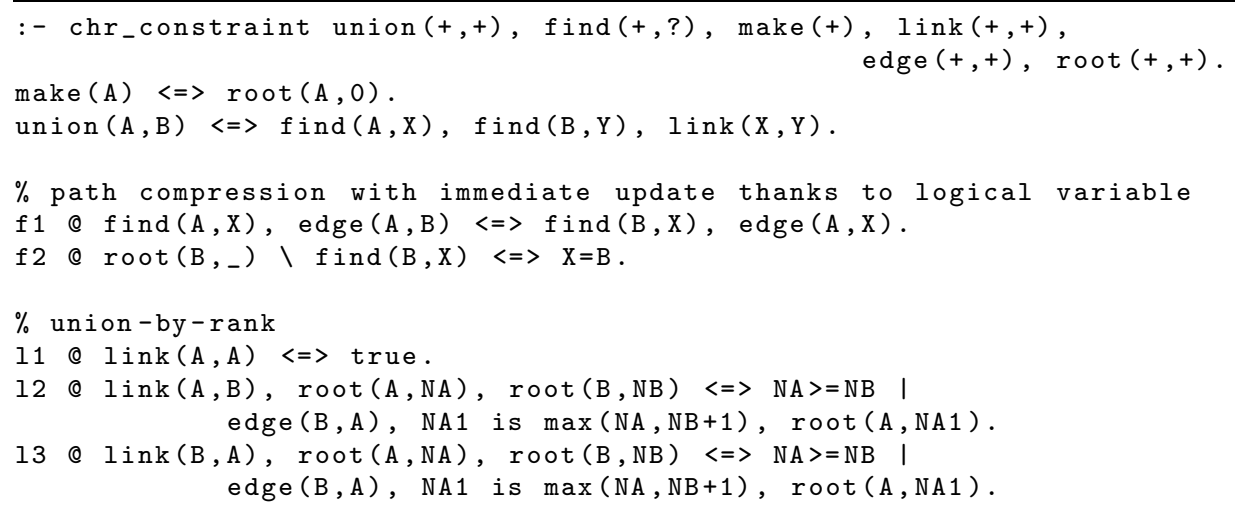

operation on average. Two techniques can be used to improve the performance of the algorithm; if combined, the average time per operation can be reduced to $O(\alpha(n))$, the inverse Ackermann function of $n$, which can be considered constant for all practical purposes. The first technique is called union-by-rank and consists of maintaining the size of each tree and modifying the union/2 operation such that it always links the smaller tree to the bigger tree. The second technique is called path compression: after every find/2 operation, all edges on the path to the root are modified so that every node on the path is linked directly to the root. Listing 5.2 gives an optimal implementation of the union-find algorithm. In Section 11.5.2 we will experimentally evaluate its time complexity. Both programs are due to Schrijvers and Frühwirth (2006).

\subsection{Dijkstra's shortest path algorithm}

In this section, we construct a readable, compact and efficient implementation of Dijkstra's shortest path algorithm and Fibonacci heaps. Instead of using natural language or pseudo-code, we immediately describe the algorithms using CHR rules.

We assume that the refined semantics (see Section 4.3) is followed. Correctness, termination, and complexity of the program as listed here crucially depend on this execution strategy.

\subsubsection{The single-source shortest path problem}

The shortest path problem (Zwick 2001) is one of the most basic, and most studied, problems in algorithmic graph theory. It appears as a sub-problem in many graph related algorithms, such as network optimization algorithms. 
Given a weighted directed graph $G=(V, E)$ and a source node $s \in V$, we are interested in the distances from $s$ to all other nodes of the graph. This is called the Single-Source Shortest Path (SSSP) problem. In the rest of this section we will use $n=\# V$ to denote the number of nodes and $m=\# E$ to denote the number of edges. We assume the weights to be nonnegative, but not necessarily integer. We also assume that the graph is connected, so $n$ is $O(m)$.

The SSSP problem with nonnegative edge weights can be solved using the classical algorithm of Dijkstra (1959). A naive implementation of this algorithm runs in $O\left(n^{2}\right)$ time, which is suboptimal for non-dense graphs.

\section{Priority queues}

Efficient implementations of Dijkstra's algorithm use a priority queue to maintain tentative distances. A priority queue is a data structure consisting of a set of item-key pairs, subject to the following operations: insert, adding a new pair to the queue; extract_min, returning and removing the pair with the minimum key; and decrease_key, decreasing the key of a given item.

Fibonacci heaps are an implementation of a priority queue that performs the insert and decrease_key operations in $O(1)$ amortized time, and extract_min in $O(\log n)$ amortized time, where $n$ is the number of elements in the queue. It is based on binomial queues (Vuillemin 1978), which implement all operations in $O(\log n)$ time. Using Fibonacci heaps (Fredman and Tarjan 1987), Dijkstra's algorithm takes $O(m+n \log n)$ time. This combination is the fastest known algorithm for solving the SSSP problem with non-negative real edge weights.

\subsubsection{Dijkstra's algorithm}

The input graph is given as $m$ edge/3 constraints: a (directed) edge from node $A$ to $B$ with weight $W$ is represented as edge $(A, B, W)$. Node names are integers in $[1 . . n]$ and weights are non-negative numbers. The query consists of a sequence of edge/3 constraints followed by one dijkstra $(S)$ constraint, where $S$ is the source node. The output of the algorithm consists of $n$ distance (X,D) constraints, where $\mathrm{D}$ is the distance from node $\mathrm{S}$ to node $\mathrm{X}$.

During the execution of Dijkstra's algorithm, each node is in one of three states: unlabeled, labeled (with a tentative distance), or scanned (and annotated with a correct distance). Initially, every node is unlabeled except for the source node which gets tentative (and correct) distance zero. The algorithm repeatedly picks a labeled node with the smallest tentative distance and scans it.

We use a global priority queue to store the labeled nodes; the item is the node name, the key is its tentative distance. When a node $\mathrm{X}$ is scanned, we store its distance $\mathrm{D}$ as a distance $(\mathrm{X}, \mathrm{D})$ constraint. The unlabeled nodes are not stored explicitly. We assume that the priority queue has a decr_or_ins(Item,Key) operation which inserts (Item, Key) into the queue if Item is not already in the 
Listing 5.3: DIJKSTRA: Dijkstra's shortest path algorithm

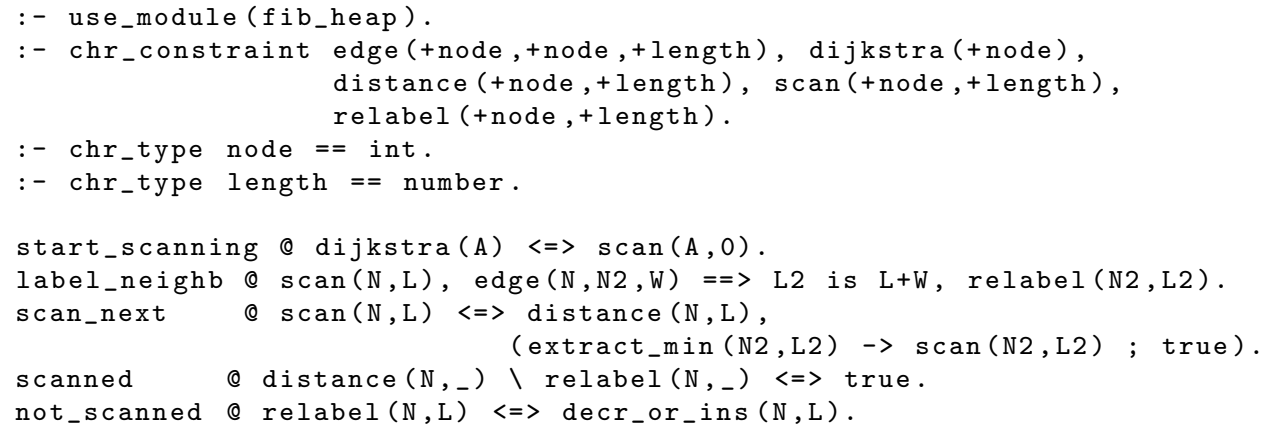

queue; otherwise it updates the key of Item if the new key Key is smaller than the original key (if it is larger, the operation has no effect). We also assume that there is an extract_min/2 operation which returns the item with the smallest key and removes it from the queue (and fails when the queue is empty).

The distance to the source node is zero. We start by scanning the source:

start_scanning $@ \operatorname{dijkstra}(A) \Leftrightarrow \operatorname{scan}(A, 0)$.

To scan a node, all its outgoing edges are examined to relabel the neighbors. The candidate label for a neighbor N2 is simply the sum of the distance L to N, and the weight $\mathrm{W}$ of the edge connecting $\mathrm{N}$ and $\mathrm{N} 2$ :

label_neighb $@ \operatorname{scan}(N, L), \operatorname{edge}(N, N 2, W)=\Rightarrow$ L2 is $L+W, \operatorname{relabel}(N 2, L 2)$.

Then we make the node scanned and scan the labeled node with the smallest tentative distance (or stop if there are no labeled nodes left):

scan_next @ $\operatorname{scan}(N, L) \Leftrightarrow \operatorname{distance}(N, L)$, (extract_min $(\mathrm{N} 2, \mathrm{~L} 2) \rightarrow \operatorname{scan}(\mathrm{N} 2, \mathrm{~L} 2) ;$ true $)$.

Relabeling works as follows: we do nothing if the neighbor is already scanned. If the neighbor node $\mathrm{N}$ is not scanned, it is either unlabeled or labeled. Using the decr_or_ins operation, $N$ gets a label if it is unlabeled and gets a new label if the candidate label $\mathrm{L}$ is smaller than its original label.

scanned @ distance $\left(\mathrm{N},{ }_{-}\right) \backslash \operatorname{relabel}\left(\mathrm{N},{ }_{-}\right) \Leftrightarrow$ true.

not_scanned @ relabel $(\mathrm{N}, \mathrm{L}) \Leftrightarrow \operatorname{decr}$ or_ins $(\mathrm{N}, \mathrm{L})$.

The full program text is given in Listing 5.3 . 


\subsubsection{Fibonacci heaps}

We use Fibonacci heaps to implement the priority queue needed in Dijkstra's algorithm.

Data structures. The items and their keys are stored as nodes in a collection of heap-ordered trees. A heap-ordered tree is a rooted tree where the key of any node is no less than the key of its parent. The number of children of a node is called its rank. Nodes can be either marked or unmarked. Root nodes are never marked. A node containing the item I with key $K$ is stored as an item $(I, K, R, P, M)$ constraint, where $R$ is its rank and $P$ is the item of its parent (or 0 if it is a root). The last $\operatorname{argument} \mathrm{M}$ is $\mathrm{u}$ if the node is unmarked and $\mathrm{m}$ if it is marked. The minimum-key pair is stored as a min/2 constraint:

keep_min $@ \min \left({ }_{-}, \mathrm{A}\right) \backslash \min \left({ }_{-}, \mathrm{B}\right) \Leftrightarrow \mathrm{A}=<\mathrm{B} \mid$ true.

Insert. Inserting a new item I with key $\mathrm{K}$ is done by adding an unmarked isolated root node and updating the minimum:

insert $\mathbb{Q} \operatorname{insert}(I, K) \Leftrightarrow \operatorname{item}(I, K, 0,0, u), \min (I, K)$.

Extract-min. Extracting the minimum node is done as follows. First we find and remove the min constraint (if there is none, the heap is empty and we fail) and the corresponding item. Then we convert the children of the minimum node to roots, which is done by the auxiliary ch2rt constraint. Finally we find the new minimum (done by the findmin constraint) and return the (old) minimum item.

$\operatorname{extr} @ \operatorname{extract} \_m i n(X, Y), \min (I, K), \operatorname{item}\left(I,,_{-},,_{-}\right)$

$\Leftrightarrow \operatorname{ch} 2 \mathrm{rt}(\mathrm{I})$, findmin, $\mathrm{X}=\mathrm{I}, \mathrm{Y}=\mathrm{K}$.

extr_empty @ extract_min $\left(_{-},{ }_{-}\right) \Leftrightarrow$ fail.

The following simpagation rule converts the children of I to roots:

c2r@ ch2rt(I) \item $\left(\mathrm{C}, \mathrm{K}, \mathrm{R}, \mathrm{I}, \mathrm{I}_{-}\right) \Leftrightarrow \operatorname{item}(\mathrm{C}, \mathrm{K}, \mathrm{R}, \mathrm{O}, \mathrm{u})$.

c2r_done Q ch2rt (I) $\Leftrightarrow$ true.

To find the new minimum, it suffices to search the root nodes:

findmin @ findmin, item $\left(I, K,,_{-}, 0,,_{-}\right)=\Rightarrow \min (I, K)$.

foundmin @ findmin $\Leftrightarrow$ true.

We want to make sure that the number of roots is $O(\log n)$ where $n$ is the number of items. The following rule links trees whose roots have the same rank, reducing the number of roots:

same_rank @ item $\left(I 1, K 1, R, 0,{ }_{-}\right)$, item $\left(I 2, K 2, R, 0,{ }_{-}\right) \Leftrightarrow K 1=<K 2$ |

item $(I 2, K 2, R, I 1, u)$, item $(I 1, K 1, R+1,0, u)$. 
Decrease-key. The decrease-key operation removes the original item constraint and calls the auxiliary constraint decr $/ 5$ if the new key is smaller than the original key (and fails otherwise).

$\operatorname{decr} @ \operatorname{decr}(I, K)$, item $(I, O, R, P, M) \Leftrightarrow K<0 \mid \operatorname{decr}(I, K, R, P, M)$. decr_nok @ $\operatorname{decr}(I, K) \Leftrightarrow$ fail.

The decr_or_ins operation calls decr/5 if the item is on the heap and the new key is smaller than the original. If the item is on the heap but the new key is larger, it does nothing; the item is inserted if it is not on the heap.

doi_d @ item(I,O,R,P,M), decr_or_ins $(I, K) \Leftrightarrow K<0 \mid \operatorname{decr}(I, K, R, P, M)$. doi_nop @ item $\left(I, 0_{-},,_{-}\right) \backslash \operatorname{decr}$ or_ins $(I, K) \Leftrightarrow K>=0 \mid$ true.

doi_insert @ decr_or_ins $(I, K) \Leftrightarrow \operatorname{insert}(I, K)$.

When a key is decreased, we may have found a new minimum:

d_min $@ \operatorname{decr}\left(I, K,,_{-},{ }_{-}\right)==>\min (I, K)$.

Decreasing the key of a root cannot cause a violation of the heap order:

d_root $@ \operatorname{decr}\left(I, K, R, 0, \_\right) \Leftrightarrow \operatorname{item}(I, K, R, 0, u)$.

If the new key is not smaller than the parent's key, there is also no problem:

d_ok @ item $\left(P, P K,,_{-},{ }_{-}\right) \backslash \operatorname{decr}(I, K, R, P, M) \Leftrightarrow K>=P K \quad$ item $(I, K, R, P, M)$.

Otherwise, we cut the violating node and make it a new root. The original parent is marked to indicate that it has lost a child.

d_prob @ $\operatorname{decr}(I, K, R, P, M) \Leftrightarrow \operatorname{item}(I, K, R, 0, u), \operatorname{mark}(P)$.

To obtain the desired time complexity, we have to make sure that when a (nonroot) node loses two of its children through cuts, it gets cut as well. This is called a cascading cut. Nodes are marked to keep track of where to make cascading cuts. The mark constraint decreases the rank of a node and marks it if necessary. A root node is never marked, an unmarked node becomes marked, and an already marked node is cut and its parent is marked (cascading cut):

m_rt @ $\operatorname{mark}(I), \operatorname{item}\left(I, K, R, 0,{ }_{-}\right) \Leftrightarrow \operatorname{item}(I, K, R-1,0, u)$.

$m_{-} u @ \operatorname{mark}(I)$, item $(I, K, R, P, u) \Leftrightarrow \operatorname{item}(I, K, R-1, P, m)$.

m_m $@ \operatorname{mark}(I), \operatorname{item}(I, K, R, P, m) \Leftrightarrow \operatorname{item}(I, K, R-1,0, u), \operatorname{mark}(P)$.

This concludes the algorithm. Listing 5.4 gives the full CHR program. For optimization, a pragma passive compiler directive (see Section 4.3.2) was added to rule c $2 r$, the m_er rule was added (to make mark/1 never-stored, cf. secton 4.4.3), and the same_rank rule was reformulated without guard. 
Listing 5.4: FIB-HEAP: Fibonacci-heap implementation of priority queues

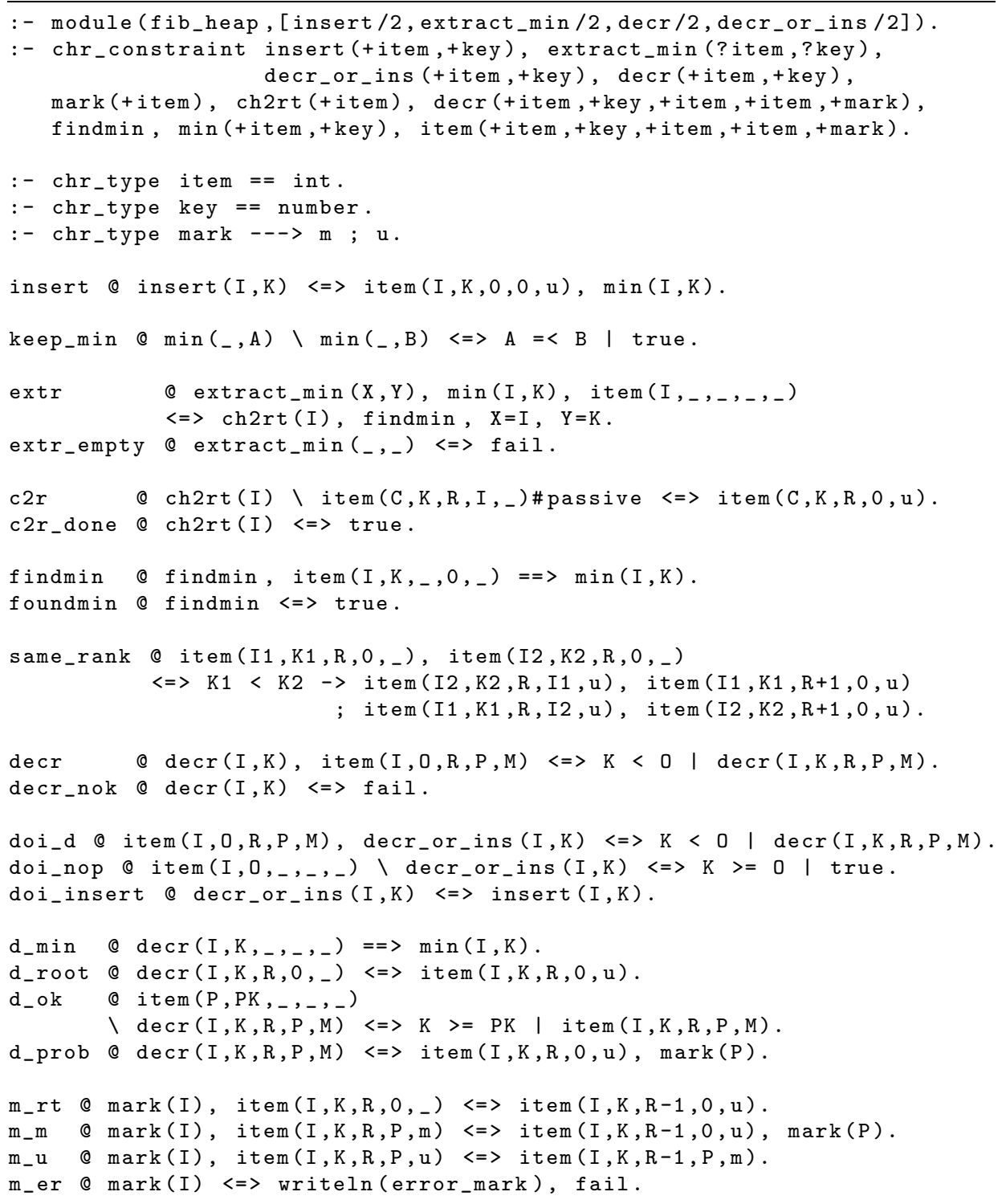




\subsubsection{Time complexity}

To obtain the desired $O(m+n \log n)$ time complexity, we depend on some properties of the CHR implementation. In Chapter 7 we will discuss the required implementation techniques. For now, we will simply assume that every rule application takes one unit of time for both programs.

(1) This assumption implies that partner constraint lookups need to be implemented in an efficient way. Specifically, we require constraint stores to allow constanttime insertion, deletion, and look-up on arbitrary argument positions. The crucial argument positions for which constant time look-ups are necessary are the following: the first argument of edge $/ 3$, the first argument of distance $/ 2$, and the first and fourth argument of item $/ 5$.

\section{Complexity of DIJKSTRA}

Dijkstra's algorithm starts with an empty heap, performs $n-1$ insert operations, $m-n$ decrease-key operations and $n$ extract-min operations. Hence, the total time complexity of the algorithm is $O(n I+m D+n E)$, where $I, D$ and $E$ are the amortized time complexities of one insert, decrease-key and extract-min operation, respectively.

\section{Complexity of FIB-HEAP}

Under the assumption that one rule application takes constant time, the analysis of Fredman and Tarjan (1987) remains valid. Insert and decrease-key take constant amortized time, and extract-min takes logarithmic amortized time.

In the original description of Fibonacci heaps by Fredman and Tarjan (1987), the linking step (corresponding to the same_rank rule) is only performed during an extract-min operation (corresponding to the extr rule), just before the finding the new minimum (findmin). In the variant presented here, the same_rank rule is triggered each time a node becomes a root or a new root is added. This does not affect the amortized time bounds, but it does improve performance in practice.

To analyze the amortized running times of the Fibonacci heap operations, we assign a potential to every possible heap configuration. The amortized time of an operation is its actual running time plus the net increase it causes in the potential. Hence, the actual time of a sequence of operations is equal to the total amortized time plus the total net decrease in potential (see Section 2.3.4). We define the potential of a heap to be the total number of trees it contains plus twice the number of marked nodes. The initial potential is zero, and the potential is always nonnegative, so the total amortized time of a sequence of operations is an upper bound on the total actual time.

Corollary 1 in (Fredman and Tarjan 1987) states that in a Fibonacci heap, nodes of rank $k$ have at least $F_{k+2}$ descendants, where $F_{i}$ is the $i$-th Fibonacci 
number (cf. Section 3.5.2). Because the Fibonacci numbers grow exponentially, this result implies that in a Fibonacci heap with $N$ items, the maximal rank of any node is $O(\log N)$, and that the number of roots is also $O(\log N)$ if no two roots have the same rank. During the execution of Dijkstra's algorithm, there can be at most $n-1$ items in the heap in the worst case, so we get a $O(\log n)$ bound on the rank and number of roots. Performing one link (i.e. finding a partner constraint for the same_rank rule and executing its body) takes constant actual time, and since one root node becomes a child node, the potential decreases by one.

Insert. The insert operation adds a new root node, increasing the potential by one minus the number of links performed. This takes actual time proportional to the number of links performed. It may then trigger the keep_min rule, which takes constant time. Hence the amortized time for the insert operation is $O(1)$.

Extract-min. The extract-min operation either fails in constant time, or it finds the minimum item in constant time. In the latter case, it first converts $O(\log n)$ children to roots, which takes $O(\log n)$ amortized time. The potential increases by the number of new roots minus twice the number of children that were marked. This is clearly bounded by $O(\log n)$. Then findmin goes through the $O(\log n)$ new roots to find the new minimum. Hence the amortized time for the extractmin operation is $O(\log n)$.

Decrease-key. If decreasing the key of an item does not violate heap order, the decrease-key operation does not affect the potential and clearly takes only constant time. In the other case, a new root is added (adding one to the potential), followed by a number of cascading cuts (rule $\mathrm{m}_{-} \mathrm{m}$, which decreases the potential by one, since it adds a root $(+1)$ and unmarks a previously marked node (-2)), and finally marking an unmarked node ( $\mathrm{m}_{-} \mathrm{u}$, adding two to the potential) or doing nothing (m_rt). So the net increase of the potential is at most three minus the number of cascading cuts. Since every cut can be done in constant time, the actual time is proportional to the number of cascading cuts. Hence the amortized time for the decrease-key operation is $O(1)$. Clearly, the "decrease-key or insert" operation also takes constant amortized time.

From the above it follows that the total time complexity of our CHR implementation of Dijkstra's algorithm with Fibonacci heaps is $O(m+n \log n)$.

\subsubsection{Conclusion}

We have presented a readable, compact, and efficiently executable CHR description of Dijkstra's algorithm with Fibonacci heaps. We have analyzed the time complexity of both algorithms under some assumptions. In the following chapters (in particular, in Chapters 7 and 11) we will show why these assumptions are 
valid. In Section 11.5.2 we will experimentally evaluate the time complexity and the constant factors of the implementation in CHR.

The Fibonacci heap data structure is quite complex, and it is rather difficult to implement correctly. Implementations in imperative languages - for example, the one by Cherkassky et al. (1996) - typically take at least some 300 lines of hard-to-understand code. Even the pseudo-code description of Fibonacci heaps given by Cormen et al. (2001) is 63 lines long. In contrast, the program we have constructed is directly executable and consists of just 22 CHR rules, or about 40 lines including declarations and whitespace.

\section{Related work}

As far as we know, this is the first implementation of Fibonacci heaps in a declarative language.

Okasaki (1996a, 1996b) and Brodal and Okasaki (1996) have constructed functional implementations of many variants of priority queues, including binomial queues (in 36 lines of Standard ML code plus 17 signature lines), leftist heaps (Crane 1972), and pairing heaps (Fredman, Sedgewick, Sleator, and Tarjan 1986) - but not Fibonacci heaps. However, these implementations all lack the decrease$k e y$ operation. They conclude that further research is needed to implement the decrease-key operation efficiently in a (purely) functional setting without increasing the bounds for the other operations (Brodal and Okasaki 1996).

Schoenmakers (1992) uses a functional notation to aid in the analysis of a number of data structures, including Fibonacci heaps. He uses a formal functional notation extended with imperative features, such as pointer and array operators. The emphasis is on formally proving correctness and amortized complexity bounds.

King (1994) has constructed a functional implementation of the simpler and asymptotically slower binomial queues (Vuillemin 1978), using about 45 lines of Haskell code (for the operations needed in Dijkstra's algorithm). King (1994) notes that Fibonacci heaps do not lend themselves to a natural functional encoding, because of their heavy usage of pointers.

(-) Imperative implementations of Fibonacci heaps usually store at least four pointers with every item in the heap: parent, left and right sibling, and one of the children. They are used for efficient access to the children of a particular node. In CHR, there is no need to store all these pointers explicitly, since the compiler automatically constructs the appropriate indexes. For example, the $\mathrm{c} 2 \mathrm{r}$ rule does a look-up on the fourth argument of item to find the children of a node. In the code generated by the CHR compiler, a hash-table (or array) index on this argument is maintained. See Chapter 7.

McAllester (1999) has introduced a pure logic programming algorithmic model, which was extended with priorities and deletion (Ganzinger and McAllester 2001). Generalizing this model to include rules with a variable priority, Ganzinger and 
McAllester (2002) construct a very compact implementation of Dijkstra's algorithm in just three rules. They theoretically construct an interpreter for their logic programming model which can run their implementation of Dijkstra's algorithm in $O(m \log m)$ time, which is worse than the $O(m+n \log n)$ implementation presented here. However, they do not provide an implementation of Fibonacci heaps (or any other priority queue) in their logic programming formalism. The priority queue used in Dijkstra's algorithm is not explicitly implemented: it is hidden in the variable priority of the neighbor (re-)labeling rule. In their theoretical construction of the interpreter, they suggest using Fibonacci heaps to implement variable priority rules.

\subsection{Hopcroft's algorithm for finite automata}

A (deterministic) finite automaton (DFA) can be tought of as a Turing machine without tape. It has a finite number of states and a transition function from (state, input symbol)-pairs to states. Starting from a start state, it takes a string of input symbols and follows the corresponding transitions. After the string has been processed, the automaton accepts or rejects the string depending on whether the last state is an accepting final state or not. The set of languages that can be recognized by finite automata is called the set of regular languages.

Any regular language can be described by means of many finite automata. As a result, one of the problems related to finite automata is finding the most succinct description of a regular language - that is, a finite automaton that recognizes the language with as few states as possible. Hopcroft (1971) described an $O(n \log n)$ algorithm for minimizing states of a DFA.

\subsubsection{Minimizing finite automata}

Let $S$ be the set of states, $I$ the set of input symbols, $\delta$ the transition function (a mapping from $S \times I$ to $S$ ) and let $F \subseteq S$ be the set of accepting final states. The choice of initial state is not important for the algorithm. Two states $s \in S$ and $t \in S$ are equivalent if starting from each state, exactly the same strings are accepted. Hopcroft's algorithm finds the equivalence classes of $S$; all equivalent states can be joined and this results in a minimal automaton.

Figure 5.1 gives the algorithm exactly ${ }^{1}$ as described by Hopcroft (1971). The output of the algorithm are the sets $B(i)$, called blocks, of equivalent states. Initially there are two blocks (equivalence classes of states): the set of accepting final states and the other states. The algorithm proceeds by partitioning the blocks by considering an input symbol $a$ and a target block $i$ : in every block, there will be

\footnotetext{
${ }^{1}$ Modulo some minor notational differences: we write $A(a, i)$ instead of $a(i)$ for the sets constructed in steps 2 and $7 \mathrm{~b}$ (to make it clear that the sets are parametrized in both $a$ and $i$ ), and we use "\" to denote set difference instead of "-".
} 
states that go to a state from the target block $i$ on input $a$ and states that don't; the difference justifies splitting the block in two. The optimal time complexity is achieved by carefully picking the pivotal target blocks in a way that minimizes the amount of work to be done while still guaranteeing that the blocks are splitted until their states are demonstrably equivalent.

A more detailed description, as well as correctness and complexity proofs can be found in Hopcroft (1971). Our main point here is that the pseudo-code of Figure 5.1 can be translated quite easily to CHR rules.

The original paper by Hopcroft (1971) contains an implementation of the algorithm in ALGOL. This implementation takes about 300 lines ( 5 pages) of ALGOL code, and the set of input symbols is hard-coded to be $\{0,1\}$ - extending the program to deal with larger input alphabets is not trivial.

\subsubsection{Hopcroft's algorithm in CHR}

In contrast, the CHR implementation only takes 17 rules or about half a page. It is shown in Listing 5.5. Unlike the ALGOL program of Hopcroft (1971), the CHR program works for an arbitrary set of input symbols.

The transition function $\delta$ is encoded in delta/3 constraints in the usual way: $\operatorname{delta}\left(s, i, s^{\prime}\right)$ means that $\delta(s, i)=s^{\prime}$. States are given by means of state $/ 1$ constraints, accepting final states by final/1 constraints, input symbols by input/ 1 constraints. The algorithm is started using the init constraint.

Comments refer to the pseudo-code steps corresponding to the CHR rules. Note that step 1 can be omitted altogether: to find elements $x$ of $\delta^{-1}(s, a)$ it suffices to look up delta $(x, a, s)$. The implementation of step 2 depends on the refined operational semantics: the first rule has to be tried before the second rule. Most of the program rather heavily depends on the refined operational semantics. The constraints $\mathrm{b} / 2$ store the sets $B(i)$ in the straightforward way: $\mathrm{b}(i, x)$ means that $B(i)$ contains $x$. Similarly, the sets $A(a, i)$ are stored in constraints of the form a $\left(a, i,,_{-}\right)$. The auxiliary constraint $\mathrm{nb}_{-} \mathrm{a} / 3$ is used to maintain the number of elements of these sets. The program corresponds in a rather straightforward way to the pseudo-code of Figure 5.1; the comments in the CHR program should make the mapping obvious.

(1) One minor difference between the pseudo-code and the CHR program is in step $7 \mathrm{~b}$ : in the CHR program, we set $B(k)=B^{\prime}(j)$ and we replace $B(j)$ by $B(j) \backslash B^{\prime}(j)$, instead of the other way around. Clearly this does not affect correctness or complexity, but it does allow a slightly more elegant implementation in CHR.

Although conciseness and readability are obviously somewhat vague notions, we would argue that the CHR program is as concise and readable as the pseudocode description on which it is based. Additionally the CHR program is executable and achieves the optimal $O(n \log n)$ time complexity. 
Step 1. $\forall s \in S, a \in I$, construct $\delta^{-1}(s, a)=\{t \mid \delta(t, a)=s\}$.

Step 2. Construct $B(1)=F, B(2)=S \backslash F$, and for each $a \in I$ and $i \in\{1,2\}$, construct $A(a, i)=\left\{s \mid s \in B(i)\right.$ and $\left.\delta^{-1}(s, a) \neq \emptyset\right\}$.

Step 3. Set $k=3$.

Step 4. $\forall a \in I$ construct $L(a)= \begin{cases}\{1\} & \text { if }|A(a, 1)| \leq|A(a, 2)| \\ \{2\} & \text { otherwise }\end{cases}$

Step 5. Select $a$ in $I$ and $i$ in $L(a)$. Stop if $L(a)=\emptyset$ for each $a$ in $I$.

Step 6. Delete $i$ from $L(a)$.

Step 7. $\forall j<k$ such that $\exists t \in B(j): \delta(t, a) \in A(a, i)$, do the following:

7a. Partition $B(j)$ into $B^{\prime}(j)=\{t \mid \delta(t, a) \in A(a, i)\}$ and $B(j) \backslash B^{\prime}(j)$.

7b. Construct $B(k)=B(j) \backslash B^{\prime}(j)$ and replace $B(j)$ by $B^{\prime}(j)$. Construct corresponding $A(a, j)$ and $A(a, k)$ for each $a$ in $I$.

7c. $\forall a \in I$ set $L(a)= \begin{cases}L(a) \cup\{j\} & \text { if } j \notin L(a) \text { and } 0<|A(a, j)| \leq|A(a, k)| \\ L(a) \cup\{k\} & \text { otherwise }\end{cases}$

7d. Set $k=k+1$.

Step 8. Return to Step 5.

Figure 5.1: Pseudo-code description of Hopcroft's algorithm

Listing 5.5: HOPCROFT: Hopcroft's algorithm for minimizing finite automata

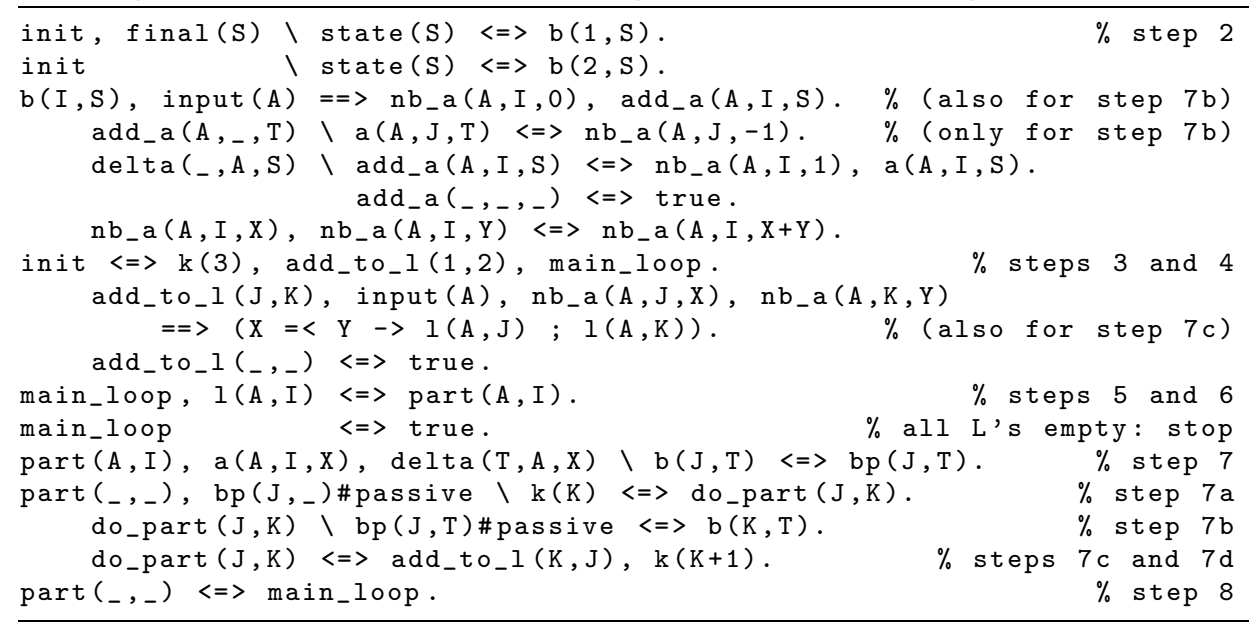


It is not trivial to achieve the optimal time complexity in current CHR systems. Typically there are two major hurdles to overcome. The first is finding the right join ordering, in particular for the rule corresponding to step 7 (cf. Chapter 9). The second hurdle is related to the performance of the constraint store data structures (cf. Chapter 7); in particular, constant-time constraint insertion, lookup, and removal is needed. When using hash-tables (cf. Section 7.2), a good hash function is needed to avoid collissions.

\subsection{Discussion and conclusion}

In this chapter we have presented elegant CHR implementations of three classic algorithms. At least in these three case studies, CHR proved to be a viable alternative to pseudo-code for describing algorithms, with the added benefit that it is directly executable. The programs discussed in this chapter are an illustration of CHR being used as a very-high-level general-purpose programming language.

Although not quite in the scope of this dissertation, we return to two new and promising language extensions of CHR that we have discussed earlier. They both enhance the usability of CHR as a programming language.

The first extension, $\mathrm{CHR}^{\mathrm{rp}}$, CHR with rule priorities (see Section 4.1.4), is based on a much more natural mechanism for execution control compared to the refined semantics that is currently commonly used. As a result, programs can be formulated in an even more concise way. A dramatic example is Dijkstra's algorithm: there is no longer a need to implement a priority queue because dynamic rule priorities can be used instead, so the programs DIJKSTRA and FIB-HEAP can be replaced by the very short program P-DIJKSTRA (see Listing 4.1 on page 60 ).

(1) Actually, implementations of $\mathrm{CHR}^{\mathrm{rp}}$ (De Koninck, Stuckey, and Duck 2008) have to maintain a priority queue to find the rule matching with the highest priority. Under the hood of a $\mathrm{CHR}^{\mathrm{rp}}$ implementation you may very well find a variant of the FIB-HEAP program.

Secondly, using aggregates (see Section 3.4.2), many auxiliary constraints and rules can be avoided. For example, in the program HOPCROFT, the constraints add $\_a / 3$ and $\mathrm{nb}_{-} \mathrm{a} / 3$ and the rules defining them are not needed if aggregates are available: the rules starting from the third rule would be replaced by a single rule:

$$
\mathrm{b}(\mathrm{I}, \mathrm{S}), \operatorname{input}(\mathrm{A}), \operatorname{exists}\left(\operatorname{delta}\left({ }_{-}, \mathrm{A}, \mathrm{S}\right)\right)==>\mathrm{a}(\mathrm{A}, \mathrm{I}, \mathrm{S}) \text {. }
$$

and in the rule where the number of a's is needed, the constraint nb_a $(A, I, X)$ would be replaced by the aggregate expression $\operatorname{count}\left(\mathrm{a}\left(\mathrm{A}, \mathrm{I},{ }_{-}\right), \mathrm{X}\right)$.

We have reached the end of the first part of this text. In the next part we discuss the compiler optimizations that are needed to get the right time and space complexity when executing CHR programs - a.o. the programs of this chapter. 
This chapter attempted to demonstrate, by investigating some case studies, that CHR is an appropriate programming language for general-purpose programming. In Part III of this dissertation we will be able to demonstrate that point in a far more rigorous and convincing manner: we show that any algorithm can be implemented in CHR with the optimal time and space complexity, not just the particular algorithms we have cared to investigate in this chapter.

However, we already wish to emphasize that despite the general complexitywise completeness result of Part III, it is still an interesting and rewarding research topic to find elegant and natural CHR implementations of classic algorithms (and also perhaps new ones). Elegantly CHR-ized classic algorithms may lead to novel variants of the original algorithm: as we have already mentioned in the beginning of this chapter, the CHR version of the union-find algorithm has lead to parallel versions of union-find (Frühwirth 2005b) and generalizations and derived algorithms (Frühwirth 2006b). 


\section{Part II \\ Optimizing Compilation of CHR}





\section{Chapter 6}

\section{Guard Reasoning}

"Value your freedom or you will lose it, teaches history. 'Don't bother us with politics', respond those who don't want to learn."

- Richard Stallman (1953-)

The abstract operational semantics $\omega_{t}$ of CHR (see Section 3.1.4) is very nondeterministic. For example, the order in which rules are applied is not specified at all. Instantiations of the abstract operational semantics - for example the refined operational semantics (see Section 4.3) - remove sources of nondeterminism and in that sense they give more execution control to the programmer.

Rule guards may be redundant under a more instantiated semantics while being necessary in the abstract semantics. Expert CHR programmers tend to remove such redundant guards. Although this improves performance, it also destroys the local character of the logical reading of CHR rules: in order to understand the meaning of a rule, the entire program and the details of the instantiated operational semantics have to be taken into account. As a solution, we propose compiler optimizations that automatically detect and remove redundant guards.

\subsection{Introduction}

All implementations of CHR should be instantiations of the $\omega_{t}$ semantics of CHR. Any of these instantiations are completely deterministic, since they compile CHR programs to instructions of a deterministic RAM machine (in the end). Although for any given version of a CHR system the execution strategy is completely fixed, CHR system developers usually do not guarantee to stick to that particular instantiation of the $\omega_{t}$ semantics in future versions, although they often do guarantee to adhere to a partial instantiation of $\omega_{t}$, for instance $\omega_{r}$. In other words, most CHR systems exclude many choices allowed by $\omega_{t}$ but at the same time they do 
not completely specify their exact deterministic operational semantics since it may change in later versions.

As we have discussed in Chapter 4, most CHR systems instantiate the refined operational semantics $\omega_{r}$ of CHR, first formally introduced by Duck et al. (2004). Briefly put, in $\omega_{r}$, the concept of an active constraint is introduced: occurrences of an active constraint are tried top-to-bottom, removed-before-kept, left-to-right, while rule bodies are executed depth-first and left-to-right. In a sense, this generalizes the standard Prolog execution strategy. Recently, other (partial) instantiations of the $\omega_{t}$ semantics have been proposed; most notably, the priority semantics $\omega_{p}$ of De Koninck et al. (2007b) — see Section 4.1.4. In this chapter we will mostly focus on the refined operational semantics.

Experienced CHR programmers know, at least to some extent, the operational semantics used by the CHR system they use. They use that knowledge to improve the performance of their program. However, the resulting CHR programs may well be no longer correct in all $\omega_{t}$ execution strategies. The dilemma CHR programmers face is the following: either they make sure their programs are valid under $\omega_{t}$ semantics, or they write programs that only work correctly under a more instantiated operational semantics. The former may result in a performance penalty, while the latter results in a program for which the logical reading of the rules no longer corresponds to their operational behavior. As explained in Section 3.1.3, CHR rules have a (linear or classical) logic reading which is local: a rule always has the same reading, whatever its context. When the specifics of the operational semantics are implicitly assumed, the locality of the logic reading is lost. For instance, under $\omega_{r}$ semantics, CHR programmers often omit the rule guards that are implicitly entailed by the rule order.

In this chapter we show how to overcome this dilemma by writing programs that are valid under the $\omega_{t}$ semantics, keeping the guards that are redundant under the $\omega_{r}$ semantics, and relying on compiler optimizations to improve performance by detecting and eliminating the redundant guards. Besides restoring the logical reading, these redundant guards - redundant under the $\omega_{r}$ semantics but needed logically and under the $\omega_{t}$ semantics - may also help other analysis tools (for example a termination checker) that are based on the abstract semantics.

Automatic code generation and source-to-source transformations are typically implemented by applying a general scheme. Such approaches often introduce many redundant guards or redundant rules. Once more, the optimizations introduced in this chapter can be used to improve the generated code for the output code.

The contributions of this chapter are the following:

1. We formalize the implicit pre-conditions of a constraint occurrence in the refined operational semantics (see Section 6.3).

2. We establish the usefulness of these pre-conditions for two compiler optimizations: guard simplification and occurrence subsumption (see Section 6.2). 
3. We describe our implementation of these optimizations (see Section 6.4). The implementation is available in the Leuven CHR system.

4. Experimental evaluation shows that our optimizations yield compact and efficient code (see Section 6.5).

5. We sketch a similar approach for the priority semantics (see Section 6.6).

\subsection{Overview}

Consider the following example CHR program, which computes the greatest common divisor using Euclid's algorithm.

\section{Example 13 (greatest common divisor).}

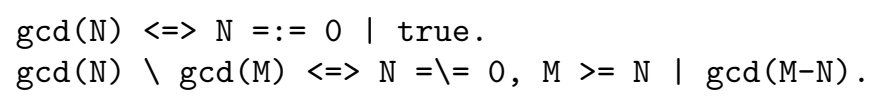

A query containing two (or more) gcd/1 constraints with positive integer arguments, will eventually result in a constraint store containing one $\operatorname{gcd}(k)$ constraint where $k$ is their greatest common divisor. For example, the query $\operatorname{gcd}(9), \operatorname{gcd}(15)$ causes the second rule to fire, resulting in $\operatorname{gcd}(9), \operatorname{gcd}(6)$. This rule keeps firing until the store contains $\operatorname{gcd}(3), \operatorname{gcd}(0)$. Now the first rule fires, removing $\operatorname{gcd}(0)$ from the store. The remaining constraint does indeed contain the greatest common divisor of 9 and 15, namely 3 .

Taking the refined semantics into account, we can simplify the above program:

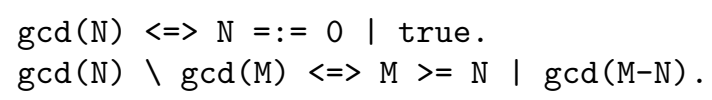

The reason is that if the second rule is tried, the guard of the first rule must have failed - otherwise the active constraint would already have been removed. Hence the condition that $N=\backslash=0$ is redundant. Under the $\omega_{t}$ semantics, this second version of the CHR program is no longer guaranteed to terminate, since applying the second rule indefinitely (which is a valid execution strategy) when the constraint store contains e.g. $\operatorname{gcd}(0), \operatorname{gcd}(3)$ results in an infinite loop.

\subsubsection{Guard simplification}

When a simpagation rule or a simplification rule fires, some or all of its head constraints are removed. As a result, for every rule $R_{i}$, we know that when this rule is tried, any non-propagation rule $R_{j}$ with $j<i$, where the set of head 
constraints of rule $R_{j}$ is a (multiset) subset of that of rule $R_{i}$, did not fire for some reason. Either the heads did not match, or the guard failed. Let us illustrate this general principle with some simple examples.

\section{Example 14 (entailed guard).}

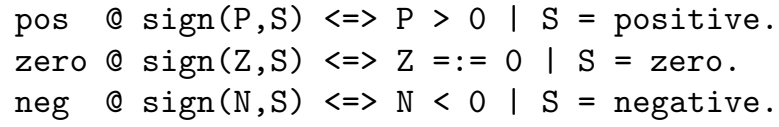

If the third rule, neg, is tried, we know pos and zero did not fire, because otherwise, the sign/2 constraint would have been removed. Because the first rule, pos, did not fire, its guard must have failed, so we know that $N \leq 0$. From the failing of the second rule, zero, we can derive $N \neq 0$. Now we can combine these results to get $N<0$, which trivially entails the guard of the third rule. Because this guard always succeeds, we can safely remove it. This results in slightly more efficient generated code, and - maybe more importantly - it might also be useful for other analyses. In this example, guard optimization reveals that all sign $/ 2$ constraints are removed after the third rule, allowing the never-stored analysis (see Section 4.4.3) to detect that sign/2 is never-stored.

\section{Example 15 (rule that can never fire).}

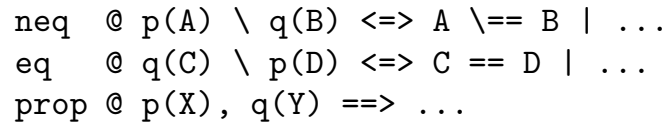

In this case, we can detect that the third rule, prop, will never fire. Indeed, because the first rule, neq, did not fire, we know that $\mathrm{X}$ and $\mathrm{Y}$ are equal and because the second rule, eq, did not fire, we know $\mathrm{X}$ and $\mathrm{Y}$ are not equal. This exhausts all possibilities, so we know the third rule can never fire.

Generalizing from the previous examples, we can summarize guard simplification as follows: If (part of) a guard is entailed by knowledge given by the negation of earlier guards, we can replace it by true, thus removing it. However, if the negation of (part of a) guard is entailed by that knowledge, we know the rule will never fire and we can remove the entire rule.

In handwritten programs, such never firing rules most often indicate bugs in the CHR program - there is no reason to write rules that cannot fire - so it seems appropriate for the CHR compiler to give a warning message when it encounters such rules. Automatic program generation and source-to-source transformations often introduce never firing rules and redundant guards, so it certainly makes sense to apply guard simplification in that context. 


\subsubsection{Head matching simplification}

Matchings in the arguments of head constraints can be seen as an implicit guard condition. By making them explicit, they can also be simplified. In other words, head matching simplification is covered by guard simplification. For example:

\section{Example 16 (head matching simplification).}

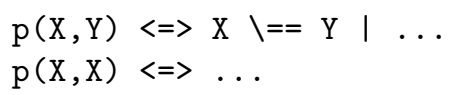

We can rewrite the head of the second rule to $\mathrm{p}(\mathrm{X}, \mathrm{Y})$ because the (implicit) condition $\mathrm{X}==\mathrm{Y}$ is entailed by the negation of the guard of the first rule. In the refined operational semantics, this does not change the behavior of the program. However, in a sense the second rule has become simpler: it imposes less conditions on the head constraint arguments. As a result, $\mathrm{p} / 2$ is now revealed to be a neverstored constraint. so more efficient code can be generated by the compiler.

\subsubsection{Type and mode declarations}

Head matching simplification can be much more effective if the types of constraints arguments are known (cf. Section 3.5.1).

For example, consider again the program SUM of Listing 3.3 (page 45). Since the first argument of the constraint sum $/ 2$ is declared to be a (ground) list, the two rules of SUM cover all possible cases and thus sum $/ 2$ is a never-stored constraint. Indeed, using the type and mode information, we can rewrite the second rule to "sum $(\mathrm{A}, \mathrm{S}) \Leftrightarrow \mathrm{A}=[\mathrm{X} \mid \mathrm{Xs}], \operatorname{sum}(\mathrm{Xs}, \mathrm{S} 2), \mathrm{S}$ is $\mathrm{X}+\mathrm{S} 2 . "$. Once more, under the $\omega_{r}$ semantics this change does not affect any computation, but since sum $/ 2$ is now clearly a never-stored constraint, the program can be compiled to more efficient (Prolog) code.

\subsubsection{Domain knowledge declarations}

We additionally allow the programmer to declare 'domain knowledge'. Suppose for instance that a Prolog fact $\mathrm{v} / 1$ is used to indicate the verbosity of the program, which can be "verbose", "normal", or "quiet". Consider the following program:

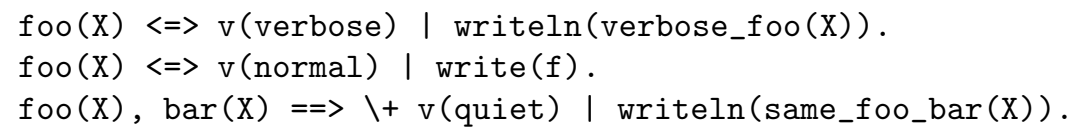

Under the refined operational semantics, the last rule can never fire. The following declaration allows the guard reasoning system to detect this:

:- chr_declaration V(verbose); v(normal); v(quiet). 
We only allow ground declarations like the above, and declarations of the form

$$
\text { :- chr_declaration predicate(X) ---> expression(X). }
$$

where all variables occurring on the right hand side should also occur on the left hand side. The left hand side should be either a CHR constraint predicate or a Prolog predicate. For example, if the Prolog predicates male/1 and female/ 1 are used in the guards of rules involving person/1 constraints, the expected behavior of those predicates could be declared as follows:

:- chr_declaration person(X) ---> male(X) ; female(X).

This declaration ensures that in the following program, the last rule is detected to never fire so it is removed:

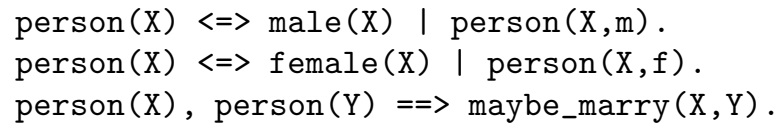

\subsubsection{Occurrence subsumption}

If the head of a rule contains multiple occurrences of the same constraint, we can test for occurrence subsumption. We know that when a certain occurrence is tried, all earlier occurrences in constraint removing rules must have failed. If the rule containing the occurrence is not a propagation rule, the same holds for earlier occurrences in the same rule.

The Leuven CHR compiler already had two optimizations that can be considered special cases of occurrence subsumption. Symmetry analysis searches for rules $R$ with a head containing two constraints $c_{1}$ and $c_{2}$ that are symmetric, in the sense that there is a variable renaming $\theta$ such that $\theta\left(c_{1}\right)=c_{2}$ and $\theta\left(c_{2}\right)=c_{1}$ and $\theta(R)=R$. In such rules, one of the $c_{i}$ 's is made passive (see Section 4.3.2). The second optimization identifies certain rules which enforce set semantics of a constraint. Such rules are of the form $c_{1}(\bar{X}) \backslash c_{2}(\bar{X}) \Longleftrightarrow$ true $\mid B$, where the arguments $\bar{X}$ are distinct variables. In such rules, the head matches all instantiations of duplicate $c$ constraints and removes one of the duplicates. In this case, $c_{1}$ can be made passive (or $c_{2}$, but it is better to keep the occurrence which immediately removes the active constraint). A more efficient constraint store can be used for $c$ if it has set semantics.

Holzbaur et al. (2001) introduced a concept called continuation optimization, which comes in two variants. Fail continuation optimization is essentially the same as occurrence subsumption, while success continuation optimization uses similar reasoning to improve the generated code for occurrences in propagation rules.

\section{Example 17 (simple case).}

$$
c(A, B), c(B, A) \Leftrightarrow p(A), p(B) \mid \text { true. }
$$


Suppose the active constraint is c $(\mathrm{X}, \mathrm{Y})$. For brevity, we say "occurrence $x$ fires" as a shortcut for "occurrence $x$ of the active constraint causes rule application". If the first occurrence does not fire, this means that either the partner constraint $\mathrm{c}(\mathrm{Y}, \mathrm{X})$ is not in the constraint store, or it is, but the guard $\mathrm{p}(\mathrm{X}), \mathrm{p}(\mathrm{Y})$ fails. The second occurrence can fire only if $c(Y, X)$ is in the constraint store and the guard $p(Y), p(X)$ succeeds. So it is impossible for the second occurrence to fire if the first one did not. (We assume conjunctions in guards to be commutative: if $\mathrm{p}(\mathrm{X}), \mathrm{p}(\mathrm{Y})$ fails, then $\mathrm{p}(\mathrm{Y}), \mathrm{p}(\mathrm{X})$ also fails.) If the first occurrence did fire, it removes the active constraint so the second occurrence is not even tried. From the above reasoning it follows that the second occurrence can be skipped, so we may change the rule to $c(A, B), c(B, A) \#$ passive $\Leftrightarrow p(A), p(B) \mid$ true.

For the rules below, in Example 18, the occurrence subsumption analysis is able to find more redundant occurrences than the earlier symmetry and set semantics analyses. Underlined occurrences can be declared passive; single underlined occurrences were already covered by the set semantics analysis, while for the double underlined occurrences, the full occurrence subsumption analysis is needed.

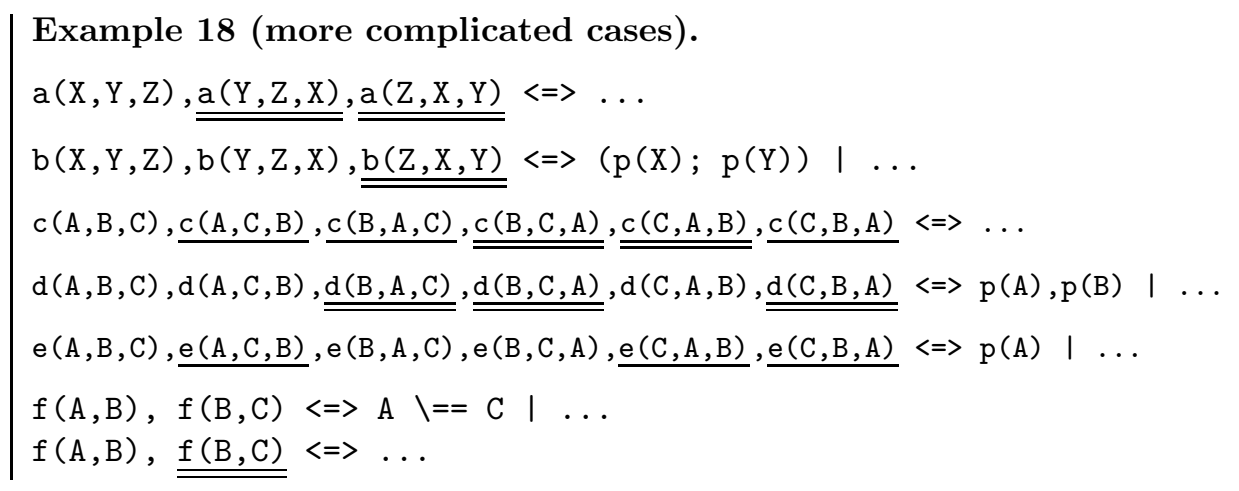

A strong occurrence subsumption analysis (partially) takes away the need for CHR programmers to write passive pragmas to improve efficiency, since the compiler is able to add them automatically if it can prove that it is a green pragma passive, i.e. it does not change the program's behavior (cf. Section 4.3.2). Because of this, the CHR source code potentially contains much less of these non-declarative operational pragmas, improving the compactness and logical readability.

Of course, not every redundant occurrence can be detected by our analysis:

Example 19 (too complicated case). Consider the last rule in the CHR version of the Sieve of Eratosthenes, the program PRIMES of Listing 3.2 on page 31. In this program, the removed occurrence of prime/1 can be declared to be passive, provided that user queries are of the form upto $(n)$, with $n \geq 1$. 
This can be justified as follows: since prime/1 constraints are added in reverse order, the guard of the absorb rule, $B \bmod A=:=0$, always fails if prime(B) is the active constraint. Indeed, for all possible partner constraints prime(A) we have $A>B>1$ because of the order in which prime/1 constraints are added, so $B \bmod A=B \neq 0$. Our implementation of occurrence subsumption lacks the reasoning capability to detect this. Not only does the current implementation lack a mechanism for the programmer to indicate which kind of queries are allowed, it does not investigate rule bodies either.

Furthermore, our entailment checker (cf. Section 6.4.1) is not able to detect a complicated entailment like $\mathrm{A}>\mathrm{B}>1 \rightarrow \mathrm{B} \bmod \mathrm{A} \neq 0$. Entailment checking is undecidable in general, so for every automated occurrence subsumption test, no matter how sophisticated, there are subsumed occurrences that are not detected.

\subsection{Formalization}

In this section we formalize the guard simplification transformation that was intuitively described above. First we introduce some additional notation for the functor/arity of constraints:

Definition 6.1 (functor) For every $C H R$ constraint $c=p\left(t_{1}, \ldots, t_{n}\right)$, we define functor $(c)=p / n$. For every multiset $C$ of $C H R$ constraints we define functor $(C)$ to be the multiset $\{$ functor $(c) \mid c \in C\}$.

We denote the $i$-th rule of a program with $R_{i}$, its head with $H_{i}$ (kept head constraints with $H_{i}^{k}$ and removed ones with $H_{i}^{r}$ ), and its guard with $g_{i}$.

\subsubsection{Implicit preconditions}

We consider rules that must have been tried (according to the refined operational semantics) before some rule $R_{i}$ is tried, calling them earlier subrules of $R_{i}$.

Definition 6.2 (earlier subrule) The rule $R_{j}$ is an earlier subrule of rule $R_{i}$ (notation: $\left.R_{j} \prec R_{i}\right)$ iff $j<i$ and functor $\left(H_{j}\right) \stackrel{\oplus}{\text { functor }}\left(H_{i}\right)$.

Now we can define a logical expression nesr $\left(R_{i}\right)$ stating the implications of the fact that all constraint-removing earlier subrules of rule $R_{i}$ have been tried unsuccessfully — the name "nesr" stands for "no earlier subrules (have fired)".

Definition 6.3 (nesr) For every rule $R_{i}$, we define:

$$
\operatorname{nesr}\left(R_{i}\right)=\bigwedge\left\{\left(\neg\left(\theta_{j} \wedge g_{j}\right)\right) \mid R_{j} \prec R_{i} \wedge H_{j}^{r} \neq \emptyset\right\}
$$

where $\theta_{j}$ is a matching substitution mapping the head constraints of $R_{j}$ to corresponding head constraints of $R_{i}$. 
If mode, type or domain knowledge information is available for head constraints of $R_{i}$, it can be added to the nesr $\left(R_{i}\right)$ conjunction without affecting the following definitions and proofs, as long as this information is correct at any given point in any derivation starting from a legal query. This information is encoded as follows:

modes - Each mode is translated to a corresponding Prolog built-in: the mode "+" yields a ground/1 condition, the mode "-" a var/1 condition, and the mode "?" represents true/0. For instance, for the constraint $c(X, Y, Z)$, the mode declaration $c(+,-, ?)$ results in the condition $\operatorname{ground}(\mathrm{X}) \wedge \operatorname{var}(\mathrm{Y})$.

types - Each type declaration results in a compound condition, based on the type definition. Take for instance the type definition for the boolean type:

:- chr_type boolean ---> true; false.

The condition for constraint $\mathrm{p}(\mathrm{X})$, whose argument is of type boolean, is: $\operatorname{var}(X) \vee($ nonvar $(X) \wedge(X=$ true $\vee X=f a l s e))$. Note that this condition explicitly distinguishes between different instantiations of the argument.

domain knowledge - The unconditional and fully ground domain knowledge is added as is. For the conditional form Pattern ---> Formula we consider all predicate occurrences in nesr $\left(R_{i}\right)$ and all the heads of $R_{i}$. For each occurrence that matches Pattern, we add the corresponding instance of Formula. For example, if the declaration $p(X)--->q(X)$ is given, and the rule under consideration is $p(f \circ o), p(A) \Leftrightarrow=$, then the information to be added is $q(f \circ o) \wedge q(A)$.

Type definitions are recursively unfolded into the formula. Unrestrained unfolding is problematic for recursive types like list( $T$ ): its leads to an infinite formula. Hence, we stop the unfolding at a fixed depth. More precisely, we stop at the highest nesting depth occurring in $R_{i}$ and its earlier subrules. As an example, consider the second rule of the SUM program (page 45). For sum (L, S), the type condition that is derived from the type declaration is the following:

$$
\left(\mathrm{L}==[] ;\left(\mathrm{L}=\left[\left.\mathrm{A}\right|_{-}\right], \text {integer }(\mathrm{A})\right)\right),(\operatorname{var}(\mathrm{S}) ; \text { integer }(\mathrm{S})) .
$$

\subsubsection{Correctness of guard simplification}

Consider a CHR program $\mathcal{P}$ with rules $R_{i}$ which have guards $g_{i}=\bigwedge_{k} g_{i, k}$. Applying guard simplification to this program means rewriting some parts of the guards to true, if they are entailed by the "no earlier subrules have fired" condition (and already evaluated parts of the guard). The entire guard is rewritten to fail, if the negation of some part of it is entailed by that condition. This effectively removes the rule. Because head matchings are made explicit, head matching simplification (Section 6.2.2) is an implicit part of guard simplification. 
Definition 6.4 (guard simplification) Applying the guard simplification transformation to a CHR program $\mathcal{P}$ (with rules $R_{i}=H_{i} \Leftrightarrow \bigwedge_{k} g_{i, k} \mid B_{i}$ ) results in a new $C H R$ program $\mathcal{P}^{\prime}=G S(\mathcal{P})$ which is identical to $\mathcal{P}$ except for the guards, i.e. its rules $R_{i}^{\prime}$ are of the form $H_{i} \Leftrightarrow g_{i}^{\prime} \mid B_{i}$, where

$$
g_{i}^{\prime}= \begin{cases}\text { fail } & \text { if } \exists k \mathcal{D} \models \operatorname{nesr}\left(R_{i}\right) \wedge \bigwedge_{m<k} g_{i, m} \rightarrow \neg g_{i, k} ; \\ \bigwedge_{k} g_{i, k}^{\prime} & \text { otherwise. }\end{cases}
$$

In the second case, the $g_{i, k}^{\prime}$ are defined by

$$
g_{i, k}^{\prime}= \begin{cases}\text { true } & \text { if } \mathcal{D} \models \operatorname{nesr}\left(R_{i}\right) \wedge \bigwedge_{m<k} g_{i, m} \rightarrow g_{i, k} \\ g_{i, k} & \text { otherwise. }\end{cases}
$$

Note that this definition is slightly stronger than the definition given in (Sneyers, Schrijvers, and Demoen 2005d), because it takes into account the left-to-right evaluation of the guard. As a result, internally inconsistent guards like X $>Y, Y>X$ can be simplified to fail, and internally redundant guards can be simplified, e.g. the condition $\mathrm{X}>=\mathrm{Y}$ can be removed from $\mathrm{X}>\mathrm{Y}, \mathrm{X}>=\mathrm{Y}$.

We now formally prove the correctness of the guard simplification optimization. First we prove a lemma which will be useful later. Intuitively it says that under the $\omega_{r}$ semantics, for every point in a derivation where a rule $r$ can directly be applied with $c$ being the active constraint, there must be an earlier execution state in which the first occurrence of $c$ is about to be checked and where all preconditions for firing rule $r$ are also fulfilled.

Lemma 6.5 If in a derivation $\sigma_{0} \longmapsto^{*} \sigma_{k}$ for $\mathcal{P}$ under $\omega_{r}$ semantics, the execution state $\sigma_{k}$ is of the form $\sigma_{k}=\left\langle\left[c \# i: j \mid A_{k}\right], S_{k}, B_{k}, T_{k}\right\rangle_{n_{k}}$, and transitions $\sigma_{k} \longmapsto$ simplify $\sigma_{k+1}$ or $\sigma_{k} \longmapsto$ propagate $\sigma_{k+1}$ are applicable, applying rule $R_{x}$, then the derivation contains an intermediate execution state $\sigma_{l}=\langle[c \# i$ : $\left.\left.1 \mid A_{l}\right], S_{l}, B_{l}, T_{l}\right\rangle_{n_{l}}$, such that $\sigma_{0} \longmapsto^{*} \sigma_{l} \longmapsto^{*} \sigma_{k}$ and for every execution state $\sigma_{m}$ with $l \leq m \leq k$, the $C H R$ store contains all partner constraints needed for the application of rule $R_{x}$ and the built-in store entails the guard of rule $R_{x}$.

Proof. Consider the execution state

$$
\sigma_{l^{\prime}}=\left\langle\left[c \# i: 1 \mid A_{l^{\prime}}\right], S_{l^{\prime}}, B_{l^{\prime}}, T_{l^{\prime}}\right\rangle_{n_{l^{\prime}}} \quad\left(\sigma_{0} \longmapsto^{*} \sigma_{l^{\prime}} \longmapsto^{*} \sigma_{k}\right)
$$

just after the last Reactivate transition that put $c \# i: 1$ at the top of the execution stack; if there was no such transition, let $\sigma_{l^{\prime}}$ be the execution state just after the Activate transition that put $c \# i: 1$ at the top of the execution stack.

Suppose at some point in the derivation $\sigma_{l^{\prime}} \longmapsto^{*} \sigma_{k}$, the built-in store does not entail the guard $g_{x}$ of $R_{x}$. Then the built-in store has to change between that point and $\sigma_{k}$, so that after the change it does entail $g_{x}$. This will possibly trigger some constraints: 
- If $c$ is triggered, then $c$ is reactivated after $\sigma_{l^{\prime}}$, which is a contradiction given the way we defined $\sigma_{l^{\prime}}$.

- If another constraint $d$ from the head of $R_{x}$ is triggered, it becomes the active constraint. Now there are two possibilities:

(a) All constraints from the head of $R_{x}$ are in the CHR store. This means eventually, either rule $R_{x}$ will be tried with $d$ as the active constraint, or another partner constraint gets triggered (but not $c$, because of how we defined $\sigma_{l^{\prime}}$, in turn maybe triggering other partner constraints, but any way $R_{x}$ will be tried with one of the partner constraints as the active constraint. Because the built-in store now does entail $g_{x}$, the rule fires and a tuple is added to the propagation history. In execution state $\sigma_{k}$, this tuple will still be in the propagation history, preventing the application of rule $R_{x}$. This is of course a contradiction.

(b) Not all constraints from the head of $R_{x}$ are in the CHR store, so some are added before $\sigma_{k}$ is reached, and a similar early-firing happens at the moment the last partner constraint is added, also leading to contradiction.

- If none of the constraints from the head of $R_{x}$ are triggered, some of them are not in the CHR store yet, because if they are all there, at least one of them should be triggered, otherwise the change in the built-in store would not affect the entailment of $g_{x}$. As a result, some of the constraints occurring in the head of $R_{x}$ have to be added before $\sigma_{k}$ is reached so we get a similar early-firing situation as above, again leading to a contradiction.

All these cases lead to a contradiction, so our assumption was wrong. This shows that during the derivation $\sigma_{l^{\prime}} \longmapsto^{*} \sigma_{k}$, the built-in store always entails the guard of $R_{x}$.

Suppose at some point in the derivation $\sigma_{l^{\prime}} \longmapsto^{*} \sigma_{k}$, the CHR store does not contain all partner constraints needed for rule $R_{x}$. Then somewhere in that derivation the last of these partner constraints $(d)$ is added to the CHR store, so all constraints needed for $R_{x}$ are in the CHR store. However, the only transition that could have added $d$ to the CHR store is Activate, which also makes $d$ the active constraint. We get an early-firing situation like above because the guard of $R_{x}$ is entailed and every partner constraint (including $c$ ) is now in the CHR store. So we get a contradiction, proving that during the derivation $\sigma_{l^{\prime}} \longmapsto^{*} \sigma_{k}$, the CHR store always contains all constraints needed for rule $R_{x}$.

To conclude our proof: we have found an execution state $\sigma_{l}$ with the required properties, namely $\sigma_{l}=\sigma_{l^{\prime}}$.

Using the previous lemma we now show that the "no earlier subrules have fired" formula nesr $\left(R_{i}\right)$ is logically implied by the built-in store at the moment the rule $R_{i}$ is applied. 
Lemma 6.6 If for a given $C H R$ program $\mathcal{P}$, the rule containing the $j$-th occurrence of the CHR predicate $c$ is $R_{c, j}$, and if there is a derivation $\sigma_{0} \longmapsto^{*} \sigma_{k}$ for $\mathcal{P}$ under $\omega_{r}$ semantics, where $\sigma_{k}=\langle[c \# i: j \mid A], S, B, T\rangle_{n}$ and rule $R_{c, j}$ can be applied in execution state $\sigma_{k}$, then we have $\mathcal{D} \models B \rightarrow \bar{\exists}_{B} \operatorname{nesr}\left(R_{c, j}\right)$.

Proof. From the previous lemma follows the existence of an intermediate execution state $\sigma_{l}(0 \leq l \leq k)$, such that for every execution state $\sigma_{m}$ with $l \leq m \leq k$, the CHR store contains all partner constraints needed for the application of rule $R_{c, j}$ and its guard is entailed by the built-in store.

To prove $\mathcal{D} \models B \rightarrow \bar{\exists}_{B}$ nesr $\left(R_{c, j}\right)$, we show that

$$
\forall R_{a} \in \mathcal{P}:\left(R_{a} \prec R_{c, j} \wedge H_{a}^{r} \neq \emptyset\right) \Rightarrow\left(\mathcal{D} \models B \rightarrow \bar{\exists}_{B} \neg\left(\theta_{a} \wedge g_{a}\right)\right)
$$

Suppose this is not the case, so assume there exists a non-propagation rule $R_{a}$ such that $R_{a} \prec R_{c, j}$ and $\mathcal{D}=B \wedge \theta_{a} \wedge g_{a}$. Since $R_{c, j}$ can be applied in execution state $\sigma_{k}$, there exists a matching substitution $\theta_{c}$ matching $c$ and constraints from $S$ to corresponding head constraints of the rule $R_{c, j}$. Because $R_{a} \prec R_{c, j}$, there is a number $o_{a}<j$ such that the $o_{a}$-th occurrence of $c$ is in rule $R_{a}$. There exists an execution state $\sigma_{m}=\left\langle\left[c \# i: o_{a} \mid A_{m}\right], S_{m}, B_{m}, T_{m}\right\rangle_{n_{m}}$ with $l \leq m<k$. From this state, a Simplify or Propagate transition can fire, applying rule $R_{a}$, because:

- all partner constraints are present in $S_{m}$;

- there exists a matching substitution $\theta$ that matches $c$ and partner constraints from the CHR store to the head constraints of of $R_{a}$, namely $\theta=\theta_{a} \wedge \theta_{c}$;

- the guard $g_{a}$ is entailed because of our assumption;

- the history does not already contain a tuple for this instance, because $R_{a}$ removes some of the constraints in its head.

But this application of $R_{a}$ removes constraints needed for the rule application in $\sigma_{k}$, because every head constraint of $R_{a}$ also appears in $R_{c, j}$. This results in a contradiction. So our assumption was false, and $\mathcal{D} \models B \rightarrow \bar{\exists}_{B} \operatorname{nesr}\left(R_{c, j}\right)$.

Now we are ready for a theorem stating that guard simplification does not affect the applicability of transitions. Correctness of guard simplification with respect to operational equivalence (see Section 3.2.1) is a trivial corollary of this theorem.

Theorem 6.7 Given a CHR program $\mathcal{P}$ and its guard-simplified version $\mathcal{P}^{\prime}=$ $G S(\mathcal{P})$. Given an execution state $\sigma=\langle A, S, B, T\rangle_{n}$ occurring in some derivation for the $\mathcal{P}$ program under $\omega_{r}$ semantics, exactly the same transitions are possible from $\sigma$ for $\mathcal{P}$ and for $\mathcal{P}^{\prime}$. In other words, $\longmapsto_{\omega_{r}, \mathcal{P}} \equiv \varlimsup_{\omega_{r}}, \mathcal{P}^{\prime}$. 
Proof. The Solve, Activate and Reactivate transitions do not depend on the actual CHR program, so obviously their applicability is identical for $\mathcal{P}$ and $\mathcal{P}^{\prime}$. The applicability of Drop only depends on the heads of the rules in the program, so again it is identical for $\mathcal{P}$ and $\mathcal{P}^{\prime}$.

If a Simplify or Propagation transition is possible for $\mathcal{P}$, this means $A=$ $\left[c \# i: j \mid A^{\prime}\right]$ and $\mathcal{D} \models B \rightarrow \bar{\exists}_{B} g_{k}$, where $k$ is the rule number of the $j$-th occurrence of $c$. According to Lemma 6.6, we now know that $\mathcal{D} \models B \rightarrow \bar{\exists}_{B}$ nesr $\left(R_{k}\right)$. The rule $R_{k}^{\prime}$ is identical to $R_{k}$ except for its guard $g_{k}^{\prime}$, so the same transition is possible for $\mathcal{P}^{\prime}$ unless the guard $g_{k}^{\prime}$ fails (while $g_{k}$ succeeds). This can only happen if for some part $g_{k, x}$ of the conjunction $g_{k}$ we have $\mathcal{D} \models \bar{\exists}_{B}$ nesr $\left(R_{k}\right) \wedge \bigwedge_{m<x} g_{k, m} \rightarrow \neg g_{k, x}$. Now we can derive a contradiction: $\mathcal{D} \models B \rightarrow \bar{\exists}_{B}$ nesr $\left(R_{k}\right)$ and $\mathcal{D} \models B \rightarrow \bar{\exists}_{B} g_{k}$ combined with the previous statement gives $\mathcal{D} \models B \rightarrow \neg \bar{\exists}_{B} g_{k}$ because of course $\forall m: g_{k} \rightarrow g_{k, m}$.

If a Simplify or Propagation transition is possible for $\mathcal{P}^{\prime}$, this means $A=$ $\left[c \# i: j \mid A^{\prime}\right]$ and $\mathcal{D} \models B \rightarrow \bar{\exists}_{B}$ nesr $\left(R_{k}\right)$. Again, assume the $j$-th occurrence of $c$ is in the $k$-th rule. The same transition is also possible for $\mathcal{P}$, unless for some $x$, $\mathcal{D} \models B \rightarrow \neg \bar{\exists}_{B} g_{k, x}$. If there is more than one of such $x$ 's, choose the smallest one, i.e. let $g_{k, x}$ be the first part of the guard conjunction that fails. Note that $\mathcal{D} \models B \rightarrow \bar{\exists}_{B} \bigwedge_{m<x} g_{k, m}$. Because $\mathcal{D} \models B \rightarrow \bar{\exists}_{B} g_{k, x}^{\prime}$, we know that $g_{k, x} \neq g_{k, x}^{\prime}$, and because of the definition of guard simplification, this can only be the case if $\mathcal{D}=\operatorname{nesr}\left(R_{k}\right) \wedge \bigwedge_{m<x} g_{k, m} \rightarrow g_{k, x}$. Again, this results in a contradiction, so the applicability of Simplify and Propagation is identical for $\mathcal{P}$ and $\mathcal{P}^{\prime}$.

Since the applicability of Default only depends on the applicability of the other transitions, it is also identical for $\mathcal{P}$ and $\mathcal{P}^{\prime}$. We showed that the applicability of any of the seven possible transitions is unchanged by guard simplification, concluding our proof.

Corollary 6.8 Under the refined operational semantics, any $C H R$ program $\mathcal{P}$ and its guard-simplified version $\mathcal{P}^{\prime}$ are operationally equivalent.

Proof. According to the previous theorem, $\iota_{\omega_{r}, \mathcal{P}} \equiv \succ_{\omega_{r}, \mathcal{P}^{\prime}}$, so all states are trivially $\mathcal{P}, \mathcal{P}^{\prime}$-joinable.

For definitions of operational equivalence and joinable states we refer the reader to (Abdennadher and Frühwirth 1999).

\subsubsection{Correctness of optimizing subsumed occurrences}

Although the occurrence subsumption optimization can be seen as a source to source transformation (inserting passive pragmas), we use a slightly different approach to define the optimization formally because the common formal definitions of CHR programs and $\omega_{r}$ derivations do not include pragmas. Instead of introducing the concept of passive occurrences in the formal refined operational 
semantics, we define occurrence subsumable occurrences and then we show that trying rule application on a subsumed occurrence is redundant. First we define the following auxiliary condition:

Definition 6.9 (neocc) Given a non-propagation rule $R_{i}$ containing in its head multiple occurrences $c_{m}, \ldots, c_{n}$ of the same constraint $c$ and other partner constraints $d$. We define for every $c_{k}(m \leq k \leq n)$ :

$$
\operatorname{neocc}\left(R_{i}, c_{k}\right)=\bigwedge\left\{\neg \theta_{l}\left(\mathrm{fc}\left(R_{i}, c_{l}\right)\right) \mid m \leq l<k, \theta_{l}\left(c_{l}\right)=c_{k}\right\}
$$

where $\mathrm{fc}\left(R_{i}, c_{l}\right)=\left(g_{i} \wedge d \wedge c_{m} \wedge \ldots \wedge c_{l-1} \wedge c_{l+1} \wedge \ldots \wedge c_{n}\right)$.

As the reader can verify, $\mathrm{fc}\left(R_{i}, c_{l}\right)$ is the firing condition for rule $R_{i}$ to fire if $c_{l}$ is the active constraint. The condition neocc $\left(R_{i}, c_{k}\right)$ describes that if the $k$-th occurrence of $c$ is tried, i.e. application of rule $R_{i}$ is tried, then the earlier occurrences inside rule $R_{i}$ must have failed (since $R_{i}$ is not a propagation rule) "neocc" stands for "no earlier occurrences (in the same rule) have fired".

We now define formally which occurrences are subsumed:

Definition 6.10 (occurrence subsumption) Given a rule $R_{i}$ as in the previous definition. We say $c_{k}(m<k \leq n)$ is occurrence subsumable iff

$$
\mathcal{D} \models \operatorname{nesr}\left(R_{i}\right) \wedge \operatorname{neocc}\left(R_{i}, c_{k}\right) \rightarrow \neg \mathrm{fc}\left(R_{i}, c_{k}\right)
$$

We now show that subsumable occurrences may be skipped (i.e. can be made passive). More formally:

Theorem 6.11 Given a CHR program $\mathcal{P}$ and an $\omega_{r}$ derivation for $\mathcal{P}$ in which an execution state $\sigma=\langle[c \# i: j \mid A], S, B, T\rangle_{n}$ occurs. If $c_{j}$ is occurrence subsumable, Simplify and Propagate transitions cannot (directly) be applied on state $\sigma$.

Proof. Suppose the Simplify or Propagate transition can be applied, firing rule $R$. Using the notation from Definition 6.9 , this means that $\mathcal{D}=B \rightarrow \bar{\exists}_{B} \mathrm{fc}\left(R, c_{j}\right)$ Also, Lemma 6.6 tells us that $\mathcal{D}=B \rightarrow \bar{\exists}_{B}$ nesr $(R)$. Because of Lemma 6.5 we know that rule $R$ has been tried for the earlier occurrences of $c$ in that rule. These tries must have failed, because $R$ is a constraint-removing rule $\left(c_{j}\right.$ is occurrence subsumable) which cannot be applied twice on the same constraints. So

$$
\forall k: m \leq k<j \Rightarrow \mathcal{D} \models B \rightarrow \neg \bar{\exists}_{B} \theta_{k}\left(\mathrm{fc}\left(R, c_{k}\right)\right)
$$

where $\theta_{k}$ are matching substitutions such that $\theta_{k}\left(c_{k}\right)=c_{j}$. In other words, we have $\mathcal{D} \models B \rightarrow \bar{\exists}_{B}$ neocc $\left(R, c_{j}\right)$. Because $c_{j}$ is occurrence subsumable, we can conclude that $\mathcal{D}=B \rightarrow \neg \bar{\exists}_{B} \mathrm{fc}\left(R, c_{j}\right)$, which results in a contradiction. So the Simplify and Propagate transitions are indeed not applicable in state $\sigma$. 


\subsection{Implementation of guard reasoning}

In this section we give a brief overview of our implementation of guard simplification and occurrence subsumption in the Leuven CHR system (see Section 3.3.4).

\subsubsection{Entailment checking}

The core component for guard reasoning is a logical entailment checker. In this section we discuss our implementation, in CHR, of such an entailment checker. This implementation is used in the guard simplification analysis to test whether one condition $B$, for example $X<Z$, is entailed by another condition $A$, for example $X \leq Y \wedge Y<Z$ - in other words, whether $A \rightarrow B$ holds. The entailment checker only considers a fragment of the host language built-ins. In particular, it does not try to discover implications of user-defined predicates, which would require a complex analysis of the host language program.

\section{Overview}

As the entailment checking problem is undecidable in general, our entailment checker is necessarily incomplete. It tries to prove that $B$ is entailed by $A$; if it succeeds, $A \rightarrow B$ must hold, but if it fails, either $A \nrightarrow B$ holds or $A \rightarrow B$ holds but could not be shown. The entailment checker is written in CHR(Prolog). When the entailment $A \rightarrow B$ needs to be checked, we call the entailment checker with the query known $(A)$, test $(B)$. Schematically, it works as follows:

1. normalize the arguments of known/1 and test/1;

e.g. apply De Morgan's laws, convert $\geq,>,<$ to $\leq$ and $\neq$

2. evaluate ground expressions;

e.g. replace known $(5 \leq 3)$ by known (fail)

3. propagate entailed information;

e.g. if you find both $\operatorname{known}(X \leq Y)$ and $\operatorname{known}(Y \leq Z)$, then add known $(X \leq Z)$

4. succeed whenever known $(B)$ is added;

5. succeed if $B$ is entailed;

e.g. test $(X \neq 3)$ is entailed by $\operatorname{known}(X \leq 0)$

6. if there is a disjunction known $\left(A_{1} \vee A_{2}\right)$ : check whether $A_{1} \rightarrow B$ and also whether $\neg A_{1} \wedge A_{2} \rightarrow B$, and succeed if both tests succeed;

7. otherwise: give up and fail. 
Listing 6.1: Entailment checker: conversion of the antecedent to normal form

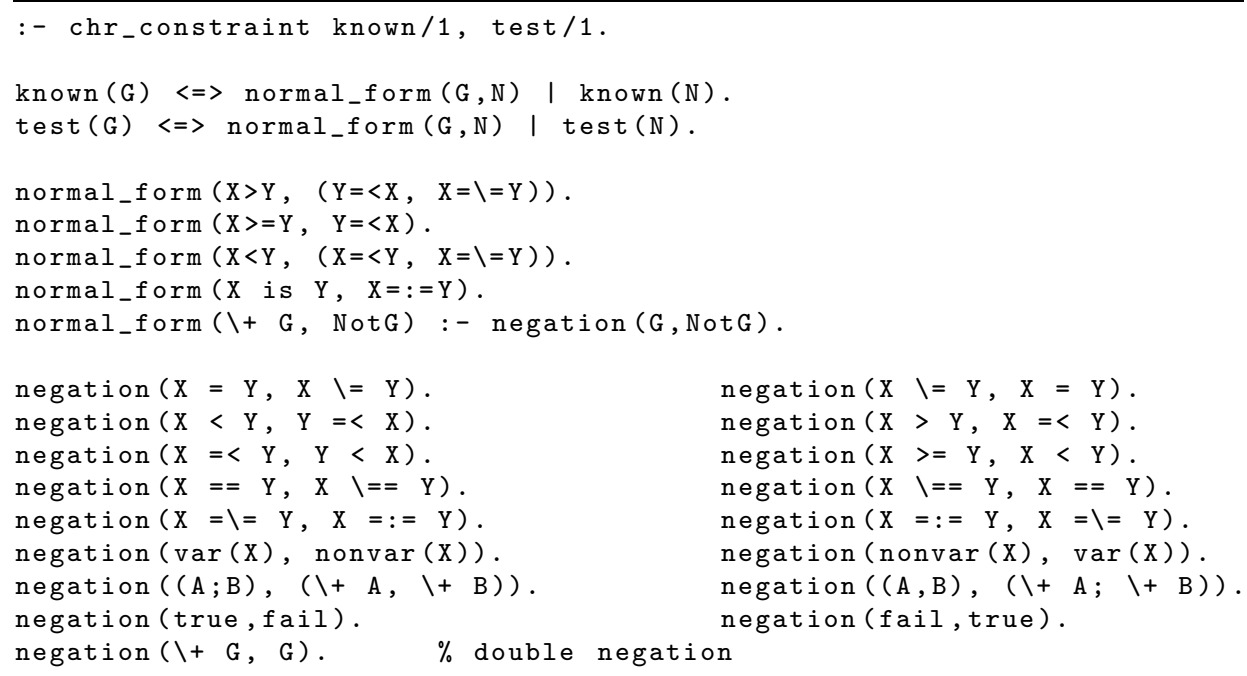

We try to postpone the expansion of disjunctions, because (recursively) trying all combinations of conditions in disjunctions can be rather costly: if $A$ is a conjunction of $n$ disjunctions of $m$ disjuncts each, there are $m^{n}$ cases that have to be checked. This is why we check entailment of $B$ before a disjunction is expanded. Conjunctions in $B$ are dealt with in the obvious way. Finally, if $B$ is a disjunction $B_{1} \vee B_{2}$, we add known $\left(\neg B_{2}\right)$ to the store and test $B_{1}$. We can stop (and succeed) if $B_{1}$ is entailed, otherwise we backtrack, add known $\left(\neg B_{1}\right)$ to the store and return the result of testing entailment of $B_{2}$.

\section{Code details}

The negation of a condition is computed in a straightforward way for host language built-ins. For example, the negation of $\mathrm{X}==\mathrm{Y}$ is $\mathrm{X} \backslash==\mathrm{Y}$, double negations cancel out, negated disjunctions become conjunctions of the negated disjuncts, and so on. For the negation of user-defined predicates p we simply use " $\backslash+\mathrm{p}$ ". Listing 6.1 shows how the normalization of known/1 and test/1 constraints is done.

Ground conditions are evaluated using rules like the following:

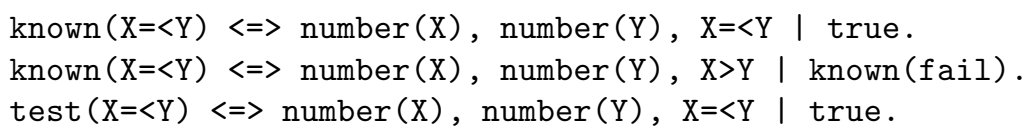

In Listing 6.2 some examples are given of rules that propagate entailed information. The idempotence rule and execution under the refined semantics is 
Listing 6.2: Entailment checker: propagation of known/1 constraints

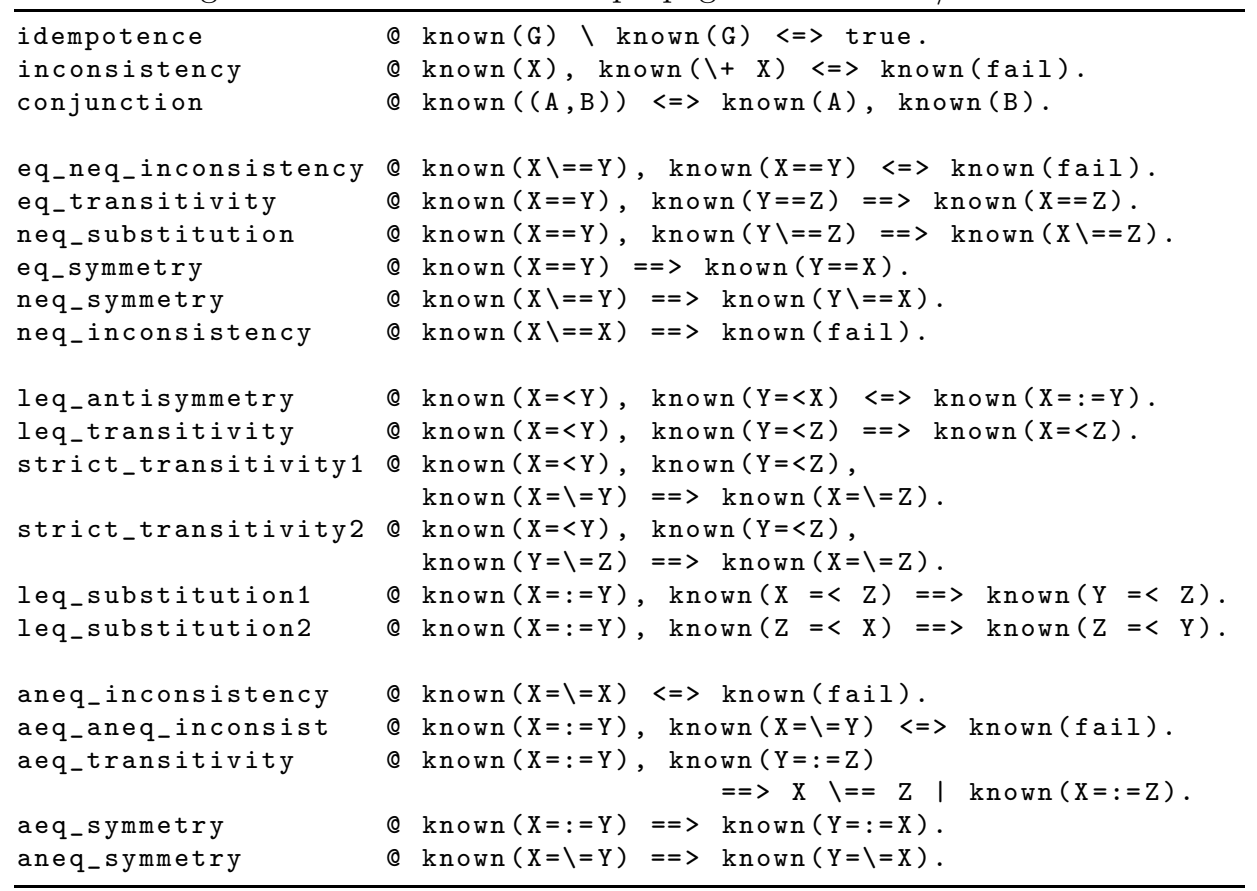

Listing 6.3: Entailment checker: part of the rest of the program

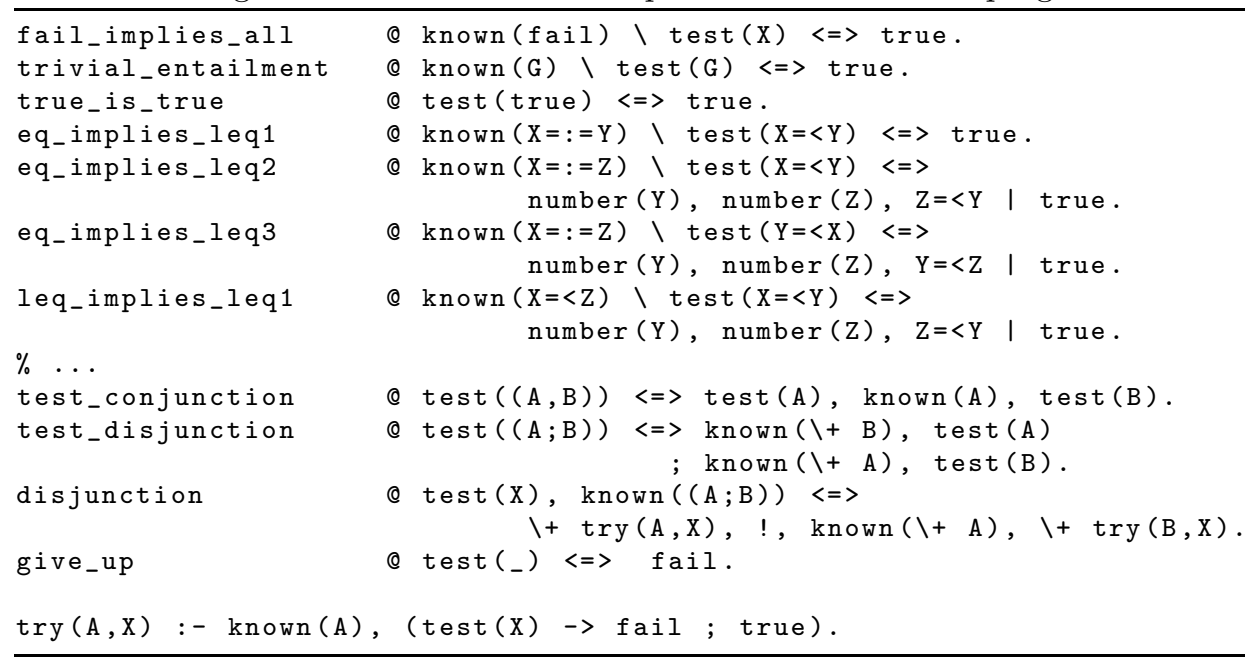


crucial for termination of this propagation phase.

A simplified version of the rest of the entailment checker is listed in Listing 6.3.

Note that Prolog disjunction in the rule body is used to check disjunctions in test $/ 1$ constraints. To deal with disjunctions in known $/ 1$ constraints, a bit of trickery is needed. We want to avoid branching until it is needed. While the propagation rules are already applied before the test constraint is activated, the disjunction rule can only be applied when the test constraint has almost reached its last occurrence. Now we use a double Prolog negation to test both disjuncts. The predicate $\operatorname{try}(A, X)$ fails if $A \rightarrow X$ can be shown, so its negation succeeds if $A \rightarrow X$ holds. By using a double negation, all propagated consequences of $A$ are automatically undone before we continue.

Disjunctions in the antecedent are the main bottleneck of the entailment checker: every disjunction potentially doubles the amount of work to be done, so the checking is potentially exponential in the input size. In the case of guard simplification, the antecedents consist of negations of guards, and guards are typically conjunctions. As a result, after normalization the antecedent consists of disjunctions (of negated conjuncts). Hence, for efficiency reasons it is important to avoid disjunction branching if possible. In addition to the above strategy of delaying disjunctions, we have added rules to simplify some common cases of redundant disjunctions. Examples of such rules are the following:

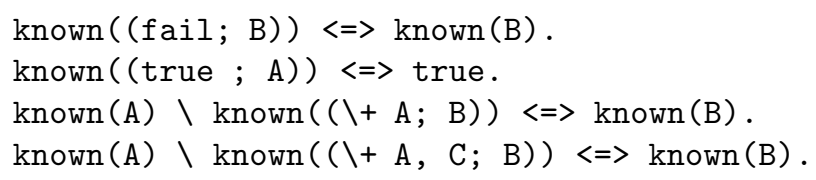

\section{Flattening}

The generic constraints known/1 and test/1 provide a conceptual simplicity in formulating and maintaining the rules of the entailment checker. However, this genericalness incurs a runtime penalty: the CHR compiler fails to efficiently index the constraints, and each active constraint has to consider (almost) all occurrences.

Because the entailment checker is one of the main performance bottlenecks in the Leuven CHR compiler, the above inefficiency is unacceptable. Fortunately, it can be mitigated with little effort by automated rule specialization. Sarna-Starosta and Schrijvers (2008) propose a technique for specializing constraints with respect to the different top-level function symbols in their arguments that rules try to match. In the current version of the entailment checker, this specialization leads to 20 versions of test $/ 1$ and 26 versions of known $/ 1$, for example 'known_==' $/ 2$, 'test_true' $/ 0$, etc.

This specialization provides indexing on the top-level function symbol for free: the CHR compiler always allocates separate indexing data structures for distinct constraint symbols. Of course, the specialization of constraints leads to the spe- 
cialization of rules, and, because many rules only apply to one specialized form, it also leads to fewer occurrences for each specialized constraint. For instance, we obtain only two occurrences for 'test_true' $/ 0$ (the first one is redundant but this is not automatically detected):

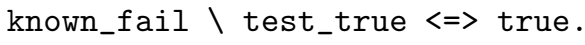

Using flattening, the entailment checker becomes roughly twice as fast.

\subsubsection{Inferring information}

The guard simplification / occurrence subsumption compilation phase rewrites every rule in the CHR program. In the rewritten rules, the redundant parts of the guard have been removed, the head matchings (an implicit part of the guard) are made as general as possible and subsumed occurrences are declared to be passive. As a result, the generated code is more efficient because redundant checks are removed, and also the next compilation phases - like storage analysis - are more effective.

Our implementation works as follows. For every rule $R_{i}$, we first compute a conjunction of inferred information. Then we use this information to transform the rule to a simpler and more efficient form.

First we make the head matchings explicit, inserting fresh variables in the arguments of head constraints as needed. For example, the rule

$c([X \mid X s], Y, Y) \Leftrightarrow \ldots \mid \ldots$

would be rewritten to the equivalent rule in head normal form:

$c(A, Y, B) \Leftrightarrow A=[X \mid X s], B==Y, \ldots \mid \ldots$

Next we iteratively construct a conjunction similar to nesr $\left(R_{i}\right)$ from Section 6.3 , containing the negations of the guards of the earlier subrules $R_{j} \prec R_{i}$. All possible substitutions have to be considered. As an example, consider the program:

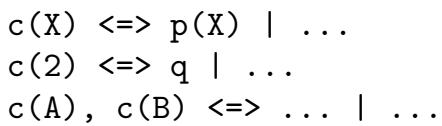

For the third rule, the following conjunction would be computed:

$(A \backslash==2 ; \backslash+q),(B \backslash==2 ; \backslash+q), \backslash+p(A), \backslash+p(B)$

Finally we add type and mode information by looking up the type and mode declarations for the head constraints of $R_{i}$, unfolding the type definitions to the nesting depth needed (see Section 6.3.1). We also add the relevant information from the domain knowledge declarations. 


\subsubsection{Using the information}

Now we can use the derived information to transform the rule. Schematically, our implementation works as follows:

1. for every part of the guard of $R_{i}$ (the $g_{i, k}$ 's from Section 6.3): check if it is entailed by the derived information and remove it if it is (i.e. replace it with true); if its negation is entailed, replace it with fail;

2. move every entailed head matching to the body if the variables in the right hand side of the matching do not occur in the guard; if they also do not occur in the body, remove the head matching;

3. produce a warning message if the guard now entails fail, or if the head matchings entail fail. This means that rule $R_{i}$ will never fire, which probably indicates a bug in the CHR program;

4. for every occurrence $c_{k}$ of a constraint that occurs more than once in $R_{i}$, compute neocc $\left(R_{i}, c_{k}\right)$ and do occurrence subsumption by checking whether $\neg \mathrm{fc}\left(R_{i}, c_{k}\right)$ is entailed by nesr $\left(R_{i}\right) \wedge \operatorname{neocc}\left(R_{i}, c_{k}\right)$, i.e. check whether occurrence $c_{k}$ can be safely set to 'passive'.

As an example of the second step, consider the rule

$$
c([X \mid X s],[], A, A,[B \mid B s]) \Leftrightarrow B>0 \mid d(X, A) \text {. }
$$

and assume the derived information entails that the first arguments of $c / 5$ is a non-empty list, the second argument is an empty list and the third and fourth argument are identical. The rule would be rewritten to

$$
c\left(Z,_{-}, A,_{-},\left[B||_{-}\right]\right) \Leftrightarrow B>0 \mid Z=\left[\left.X\right|_{-}\right], d(X, A) .
$$

\subsection{Experimental results}

In order to quantify the efficiency gain obtained by guard simplification and occurrence subsumption, we have measured the performance of several CHR benchmarks, both with and without the optimization. Before we discuss the benchmarks, we first take a look at the code the compiler generates for an example CHR program, and how this code is improved by guard simplification.

\subsubsection{Generated code comparison}

Consider the following fragment from a prime number generating program ${ }^{1}$ :

\footnotetext{
${ }^{1}$ taken from the CHR web site: http://www.cs.kuleuven.be/ d dtai/projects/CHR/
} 


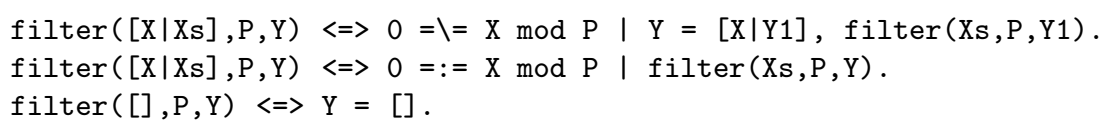

The CHR compiler (without guard simplification) generates general code for the filter $/ 3$ constraint. Because no information is known about the arguments of filter $/ 3$, the compiled code has to take into account variable triggering and the possibility that none of the rules apply and the constraint has to be stored. Following the compilation scheme explained in Section 4.2 we get:

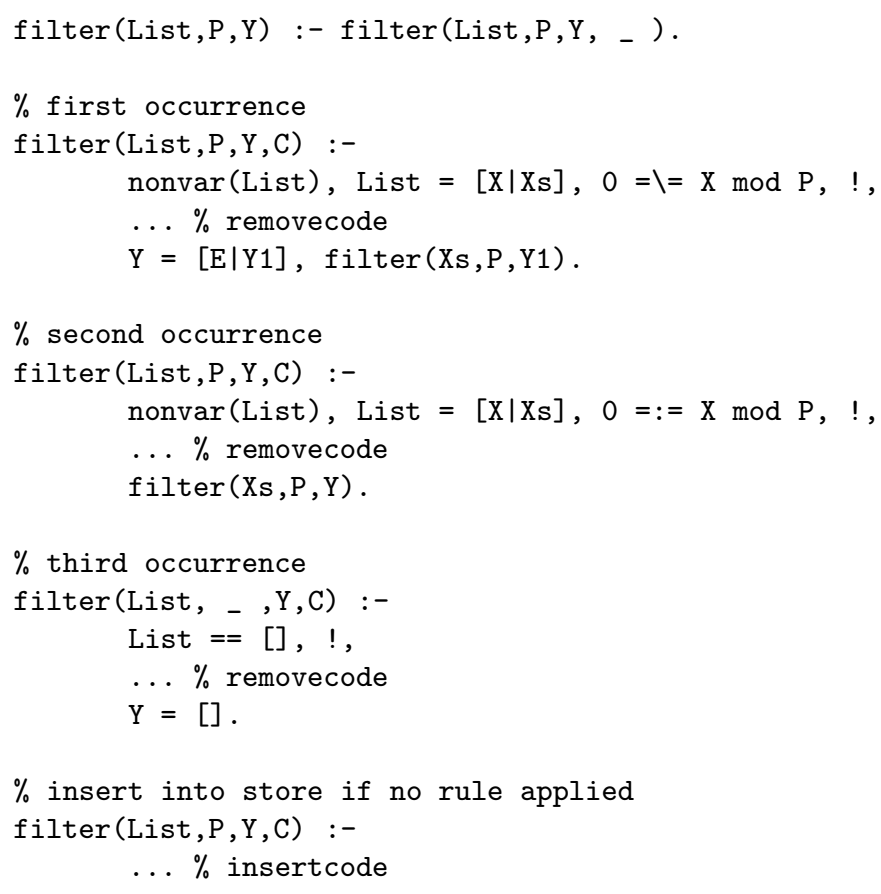

If we enable guard simplification, the guard in the second rule is removed, but this on itself does not improve efficiency considerably. A much more significant efficiency improvement can be obtained by adding type and mode information.

In this example, the programmer intends to call filter $/ 3$ with the first two arguments ground, while the third one can have any instantiation. The first and the third argument are lists of integers, while the second argument is an integer. So we add the following type and mode declaration:

$$
\text { :- constraints filter(+list(int), +int,?list(int)). }
$$

Using this type and mode information, guard simplification now detects that all possibilities are covered by the three rules. The guard in the second rule can be 
removed, so the filter $/ 3$ constraint with the first argument being a non-empty list is always removed after the second rule. Thus in order to reach the third rule, the first argument has to be the empty list - it cannot be a variable because it is ground and it cannot be anything else because of its type. As a result, we can drop the head matching in the third rule:

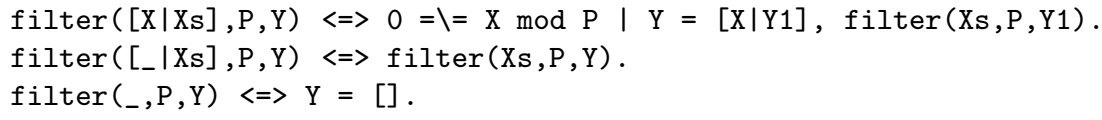

This transformed program is compiled to more efficient code, because neverstored analysis detects filter $/ 3$ to be never-stored after the third rule. The generated $\operatorname{code}^{2}$ for the guard simplified program is considerably simpler:

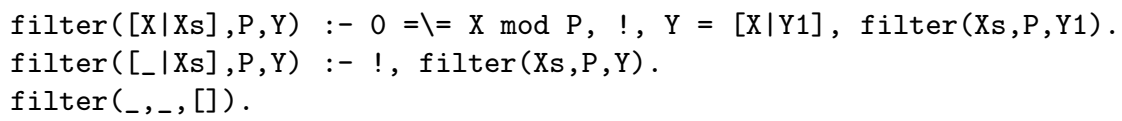

\subsubsection{Guard simplification results}

Table B.1 gives an overview of the results of running a set of benchmarks ${ }^{3}$ with and without guard simplification.

We have measured similar results (Sneyers, Schrijvers, and Demoen 2005d) in hProlog. The only significant difference with the results presented here, is the amount of run time improvement caused by adding mode information. In hProlog, this improvement is typically 20 to 30 percent, while in SWI-Prolog, it can be 50 to 70 percent. The reason is that the nonvar/1-test and other redundant code which is removed when the argument is declared to be ground - is handled much more efficiently by hProlog.

\section{Discussion}

The first benchmark, SUM, computes the sum of the elements of a list of 10000 numbers (all 1), and is repeated 500 times. We have discussed this example in Section 6.2.3. If type and mode declarations are provided, guard simplification moves the head matching to the body, enabling never-stored analysis to remove redundant code to add sum $/ 2$ to the constraint store. No performance difference could be measured between the resulting compiled program ${ }^{4}$ :

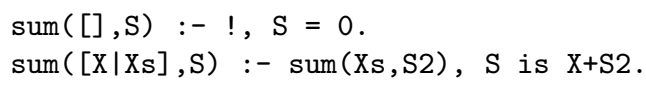

and the almost identical handwritten Prolog code:

\footnotetext{
${ }^{2}$ For readability, variables have been renamed in the generated code shown here.

${ }^{3}$ These benchmarks are available at http://www.cs.kuleuven.be/ toms/Research/CHR/.

${ }^{4}$ The results are similar for a tail-recursive version of sum $/ 2$.
} 


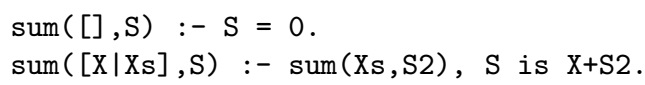

The second benchmark is an example of how guard simplification can, in a way, make mode information redundant. The CHR-program looks like this:

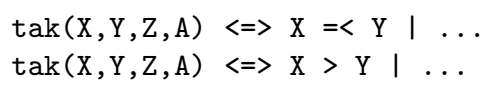

The first three arguments are supposed to be ground. If this mode information is given, the possibility of variable triggering can be excluded. However, even without mode information, guard simplification removes the guard in the second rule. As a result, the constraint is detected as being never-stored, also excluding the possibility of variable triggering. In this case, the generated code is identical to the handwritten Prolog code. The guard X $>\mathrm{Y}$ is removed because it is (entailed by) the negation of $\mathrm{X}=<\mathrm{Y}$. When $\mathrm{X}=<\mathrm{Y}$ 'only' fails, we know $\mathrm{X}$ and $\mathrm{Y}$ are ground terms evaluating to numbers, and $\mathrm{X}>\mathrm{Y}$. If in some other host language, the guard $\mathrm{X}=<\mathrm{Y}$ fails if its arguments are not instantiated to ground arithmetic terms - instead of resulting in some fatal error message or exception, as in Prolog - then its negation would have to be adjusted in the implementation of the entailment checker (cf. Listing 6.1). The negation of $\mathrm{X}=<\mathrm{Y}$ could for instance be changed to $(X>Y ; \backslash+\operatorname{number}(X) ; \backslash+\operatorname{number}(\mathrm{Y}))$. In that case, guard simplification would not remove the guard of the second rule, except when mode and type information is given.

-1. The reader may wonder: "What if $\mathrm{X}$ or $\mathrm{Y}$ are variables? Could not the transformed second rule, without a guard, fire then, while the original rule would not?" The answer is no, because trying the first guard would result in a fatal error message (at least in the Prolog systems we know), both in the original program and in the transformed program.

In the third benchmark, nrev, a list of length 30 is reversed 50000 times using the classic naive algorithm. Except for some redundant cuts, the generated code:

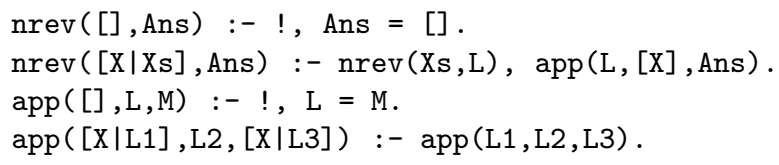

is essentially identical to the handwritten Prolog program:

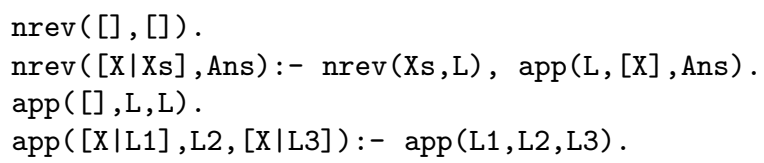


The example in Section 6.5.1 is a fragment from the fourth benchmark, cprimes, which computes the first 100,000 prime numbers. The last benchmark, df search, performs a depth-first search on a large tree. In both cases, the generated code for the guard simplified version with mode and type information is again essentially identical to the handwritten Prolog code.

\section{Conclusion}

Overall, for these benchmarks, the net effect of the guard simplification transformation - together with never-stored analysis and use of mode information to remove redundant variable triggering code - is cleaner generated code which is much closer to what a Prolog programmer would write. As a result, a major performance improvement is observed in these benchmarks.

Naive compilation causes CHR programs to have a relatively low performance compared to native host language (Prolog) alternatives. As a result, CHR programmers usually write auxiliary predicates in Prolog instead of formulating them directly in CHR. Thanks to guard simplification and other analyses, the programmer can now simply implement everything as CHR rules, relying on the compiler to generate efficient code. Mixed-language programs often use inelegant interface constructs, like rules of the form $\mathrm{foo}(\mathrm{X}) \backslash \operatorname{getFoo}(\mathrm{Y}) \Leftrightarrow \mathrm{Y}=\mathrm{X}$, to read information from the constraint store in the host language parts when this information is needed. Host language interface constraints like getFoo/1 can be avoided by writing the entire program in CHR. Thanks to (amongst others) guard simplification, this can be done without performance penalty.

\subsubsection{Occurrence subsumption results}

Table B.2 shows the results of occurrence subsumption for the rules of Example 18. Occurrence subsumption seems to result in a substantial performance improvement, if there are subsumable occurrences (which is of course true for these benchmarks). Occurrence subsumption also reduces the size of the generated code, by eliminating entire clauses. This size reduction is more pronounced than that caused by guard simplification, unless of course - as in the case of benchmarks from the previous section - guard simplification reveals the never-stored property, which also allows a substantial reduction of the generated code.

\subsection{Guard reasoning under the $\omega_{p}$ semantics}

Guard reasoning can also be applied in the context of different operational semantics. In this section we consider the priority semantics $\omega_{p}$ introduced by De Koninck et al. (2007b). The programmer assigns a priority to every rule. The $\omega_{p}$ semantics (see Section 4.1.4) is an instantiation of $\omega_{t}$ which ensures that 
of all applicable rules, the one with the highest priority is applied first. Priorities are strictly positive integer numbers, where smaller numbers indicate higher priority.

Consider the gcd program of Example 13, executed under $\omega_{p}$ semantics, and annotated with the following (rather contrived) dynamic priorities:

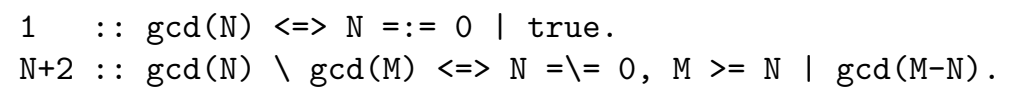

where we assume the arguments of gcd/1 to be ground positive integers. In this case, the entire guard of the second rule is redundant. The reasoning is as follows. The first rule takes priority over the second rule, so we can derive that if the second rule is applicable, the arguments of both head constraints must be different from zero. Now suppose we have the constraints $\operatorname{gcd}(\mathrm{A})$ and $\operatorname{gcd}(\mathrm{B})$ and the second rule is applicable, with matching $\theta=\{N / A, M / B\}$, and priority $A+2$. Suppose that $\mathrm{M}<\mathrm{N}$, so $B<A$. The matching $\theta^{\prime}=\{N / B, M / A\}$ has a lower priority $B+2<A+2$, so the second rule cannot be applicable with matching $\theta$. From this contradiction we can derive that $\mathrm{M}>=\mathrm{N}$ should always hold when the priority semantics allows the rule to be applicable. So under the priority semantics $\omega_{p}$, the following simplified program is equivalent to the original program:

$$
\begin{array}{ll}
1 & :: \operatorname{gcd}(\mathrm{N}) \Leftrightarrow \mathrm{N}=:=0 \mid \operatorname{true} . \\
\mathrm{N}+2 & :: \operatorname{gcd}(\mathrm{N}) \backslash \operatorname{gcd}(\mathrm{M}) \Leftrightarrow \operatorname{gcd}(\mathrm{M}-\mathrm{N}) .
\end{array}
$$

We give two more examples to illustrate how we can reason about guards under the $\omega_{p}$ semantics. The only essential difference is in the construction of the "no earlier subrules have fired" condition.

\section{Example 20 (static priorities).}

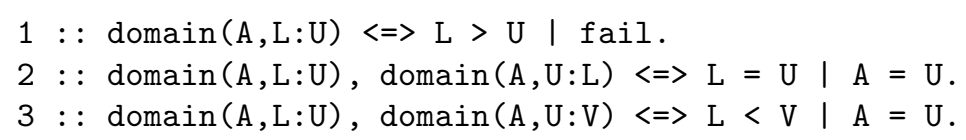

The first rule removes domain/2 constraints with an empty domain (lower bound strictly larger than upper bound). When the second rule is tried, we know the first rule is not applicable because of the priorities. So for the second rule, we know that $\neg(L>U)$ and also that $\neg(U>L)$, because otherwise one of the head constraints would have been removed by the first rule. Now we have $\neg(L>U) \wedge \neg(U>L) \leftrightarrow$ $L \leq U \wedge U \leq L \leftrightarrow L=U$, so the guard of the second rule is redundant. Now for the third rule, we know that $L \leq U$ and $U \leq V$ because of the first rule, and also that $L \neq V$ because of the second rule. Hence $L<V$ and the guard of the third rule is also redundant. 


\section{Example 21 (dynamic priorities).}

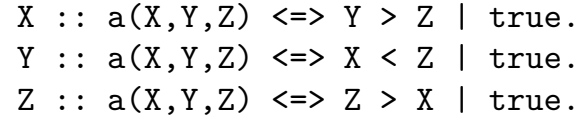

We can derive that the last rule can never fire. The reasoning is as follows. When we try the last rule for a given a/3 constraint, the first rule was not applied earlier because it would have removed the constraint. Either the first rule was not applied because the priorities allow non-application (so $Z \leq X$ ), or it was not applied because the guard failed (so $\neg Y>Z$ ). So from inspecting the first rule, assuming the last rule can be applied, we can derive that $Z \leq X \vee Y<Z$. Similarly, from inspection of the second rule, we can derive that $Z \leq Y \vee Z<X$. Now if the last rule is applicable, its guard should hold, so $Z>X$. It is easy to see that this is inconsistent with the two derived formulae, so we can conclude that the last rule is redundant and may be removed.

In future work we plan to formalize and implement guard reasoning under $\omega_{p}$ semantics.

\subsection{Conclusion}

By reasoning about guards and the operational semantics under which the program will be executed, we can automatically identify redundant guards and redundant rules. As a result, a CHR programmer can write a correct program under the general $\omega_{t}$ semantics, and the compiler will convert it to a more efficient program which is equivalent under a particular instance of $\omega_{t}$ (for example $\omega_{r}$ or $\omega_{p}$ ). Type and mode declarations can also be taken into account.

In order to achieve higher efficiency, CHR programmers often write parts of their program in Prolog if they do not require the additional power of CHR. They no longer need to write mixed-language programs for efficiency: they can simply write the entire program in CHR. Non-declarative auxiliary "host language interface" constraints like getFoo/1 (see Section 6.5.2) can be avoided.

\subsubsection{Related work}

Guard simplification is somewhat similar to switch detection in Mercury (Henderson, Somogyi, and Conway 1996). In Mercury, disjunctions - explicit or implicit (multiple clauses) - are examined for determinism analysis. In general, disjunctions cause a predicate to have multiple solutions. However, if for any given combination of input values, only one of the disjuncts can succeed, the disjunction does not affect determinism. Because they superficially resemble switches in the C programming language, such disjunctions are called switches. Switch detection 
checks unifications involving variables that are bound on entry to the disjunction and occurring in the different branches. In a sense, this is a special case of guard simplification, since guard simplification considers other tests as well, using a more general entailment checking mechanism. Guard simplification analysis can be used to remove redundant guard conditions on the source level, because CHR rules are committed-choice. It is harder to express the switch detection optimization as a source to source transformation for Mercury programs.

Guard simplification and occurrence subsumption can be combined into one analysis. In an intermediate representation, there is a separate copy of each rule for every constraint occurrence $c$, where all heads except $c$ are passive. This representation is closer to the generated Prolog code, where each occurrence gets a separate clause in which (after matching the partner constraints) the rule guard and body are duplicated. From this viewpoint, guard simplification is simplifying the guards of all copies of a certain rule at once, while occurrence subsumption is simplifying the guard of one specific copy to fail, removing that copy. A stronger and more general optimization can be obtained by simplifying the guard of each copy separately. This optimization can no longer be expressed as a pure source to source transformation. We have elaborated that approach in (Sneyers, Schrijvers, and Demoen 2005b). While reasoning on the level of constraint occurrences is stronger, it is also computationally more expensive and specific to the refined semantics, which has the concept of active occurrences.

Occurrence subsumption is essentially the same as fail continuation optimization (Holzbaur, García de la Banda, Jeffery, and Stuckey 2001; Holzbaur, García de la Banda, Stuckey, and Duck 2005), although our implementation performs much more complex implication reasoning, resulting in a stronger optimization than that of (Holzbaur, García de la Banda, Jeffery, and Stuckey 2001; Holzbaur, García de la Banda, Stuckey, and Duck 2005). The related concept of success continuation optimization (Holzbaur, García de la Banda, Stuckey, and Duck 2005) was explored in (Sneyers, Schrijvers, and Demoen 2005b). The Leuven CHR system currently implements a weak form of success continuation optimization: head matchings are taken into account to skip non-matching occurrences in the continuation of propagation rules. This could be generalized by taking into account all information that can be derived by guard reasoning.

\subsubsection{Future work}

Our current entailment checker can only deal with a limited number of Prolog built-ins. Using domain knowledge declarations, properties of user-defined Prolog predicates can be declared to enhance the capabilities of the entailment checker. The expressivity of such declarations is still fairly limited, and such declarations have to be added manually by the programmer. We see two ways for substantial further improvement. Firstly, the entailment checker could statically evaluate a call to a Prolog predicate to determine its success or failure. Here a conservative 
approach is essential as the pitfalls of side effects and non-termination must be avoided. Secondly, we may derive a solver for the Prolog predicate from its logic program definition with the techniques of (Abdennadher and Rigotti 2005). We conjecture that the latter leads to stronger results than meta-interpretation, but at a greater computational cost.

It would be interesting to explore a generalization of guard simplification that not just removes redundant conjuncts, but also replaces computationally expensive conditions by cheaper ones. For example, consider this program:

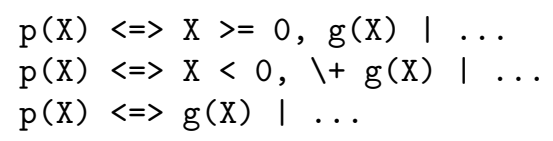

If $\mathrm{g} / 1$ is a predicate that takes a very long time to evaluate, we could change the guard of the last rule to $X<0$. This change can be justified by noting that the "no earlier subrules have fired" condition $\neg(X \geq 0 \wedge g(X)) \wedge \neg(X<0 \wedge \neg g(X))$ entails the equivalence $g(X) \leftrightarrow X<0$.

When there are many earlier subrules to consider in the guard simplification analysis, the performance of our current implementation becomes an issue. Rules with many shared head constraints are an even bigger performance issue, because of the combinatorial blowup caused by constructing all possible mappings from the head of an earlier subrule to the current rule head. If a constraint $c$ occurs $n$ times in the head of an earlier subrule, and $m(\geq n)$ times in the current head, there are $\frac{m !}{(m-n) !}$ conditions to be added to the nesr conjunction. In future work we hope to further improve the scalability of our implementation.

The information entailed by the failure and success of guards, used here to eliminate redundant guards and rules, would also be useful in other program analyses and transformations. Of particular interest is the generation of specialized code for individual constraint calls in rule bodies. Taking into account the success and failure leading up to this call, stronger guard simplification may be performed than in the general case.

Finally, an interesting area for future work is the formalization and implementation of guard reasoning for the priority semantics, as we mentioned in Section 6.6. The relation between guards and rule priorities needs further investigation: perhaps a sort of reverse reasoning can be used to simplify priorities given the guards. In this way, it could be possible to replace dynamic priorities by static priorities or to execute part of a program under the more efficient refined semantics, perhaps by adding some guards. One complication is that in the refined semantics, constraints are processed depth-first from left to right, while the priority prescribes batch processing (always Introduce before Apply). 


\title{
Chapter 7
}

\section{Indexing Techniques}

\begin{abstract}
"The required techniques of effective reasoning are pretty formal, but as long as programming is done by people that don't master them, the software crisis will remain with us and will be considered an incurable disease. And you know what incurable diseases do: they invite the quacks and charlatans in, who in this case take the form of Software Engineering gurus."
\end{abstract}

— Edsger Dijkstra (1930-2002)

In a naive implementation of CHR, the constraint store could simply be implemented as one big list. The performance of such a system would clearly be very poor. There are two main areas where more advanced data structures are needed. Firstly, when host language built-ins are executed (i.e. the Solve transition in the $\omega_{r}$ semantics, cf. Section 4.3), it has to be determined which CHR constraints are affected and thus have to be reactivated. Usually only few CHR constraints will be affected, so it is important to avoid having to consider all constraints. Secondly, constraint occurrence sub-procedures search for applicable rules by finding matching partner constraints. If there are $k$ partner constraints, and for every partner constraint, $O(n)$ constraints are considered before a matching one is found, the cost of finding an applicable rule is already $O\left(n^{k}\right)$. It should not surprise us that, even in efficient implementations of CHR, for many programs the cost of finding applicable rules is the number one performance bottleneck.

This chapter briefly discusses some indexing techniques that have been used in CHR implementations in order to improve performance on both of the above fronts. In Chapter 9 we will focus on the related problem of join ordering - finding a good order for the partner constraint lookups (in case there is more than one partner constraint). The join ordering determines the kind of indexes that need to be maintained. In this chapter we assume that the join ordering has already been determined. 


\subsection{Attributed variables}

In CHR(Prolog) systems, the only built-in constraint is Prolog unification. A rule that was not applicable before may become applicable after a unification. For example, the rule $c(X, X) \Leftrightarrow$ true is not initially applicable given the constraint $\mathrm{C}(\mathrm{A}, \mathrm{B})$, but after the unification $\mathrm{A}=\mathrm{B}$, it is. In order to find constraints that are affected by unifications quickly, attributed variables were introduced (Holzbaur 1992; Holzbaur and Frühwirth 1999; Demoen 2002).

Attributed variables are logical variables that are marked with an arbitrary Prolog term. Just after a unification occurs that involves an attributed variable, the user-definable predicate attr_unify_hook/2 is called.

Variables that occur in CHR constraints are turned into attributed variables. They are marked with a list of suspension terms (cf. Section 4.2.1) of all the constraints in which they occur. We define the predicate attr_unify_hook/2 such that it calls the reactivation goal (stored in the Continuation field of the suspension terms, cf. Table 4.1) for every constraint that is involved.

Using attributed variables, reactivation of triggered constraints can be implemented in an efficient way. Furthermore, the suspension lists that are maintained in every variable can also be used for efficient partner constraint lookup.

Example 22 (lookup via attributed variable). Consider the last rule of the LEQ program of Listing 3.1 (page 31):

transitivity @ leq $(X, Y), \operatorname{leq}(Y, Z)==\operatorname{leq}(X, Z)$.

In the occurrence sub-procedure for the first occurrence in this rule, where leq $(X, Y)$ is the active constraint, we look for a matching partner constraint of the form leq $\left(Y,{ }_{-}\right)$. Now instead of iterating through the list of all $(\mathrm{leq} / 2)$ constraints, we can also access the attribute of the variable $Y$, which contains a list of all constraints in which it occurs. This will result in a potentially much smaller list that still contains all the matching partners.

\subsection{Hash tables}

Attributed variables only provide a partner constraint lookup mechanism as long as the partner constraint contains a variable which is shared with the active constraint or with another, already matched partner constraint. In particular, for constraints that are ground (do not contain any variables), the lookup mechanism falls back on the naive iteration over all constraints, which takes linear time. 


\subsubsection{Ground hash tables}

If the arguments of a constraint are known (by inference or by declaration) to be ground, efficient data structures can be used to allow lookups via such arguments. Holzbaur et al. (2005) have proposed the use of a balanced tree for the lookup of constraints via known ground arguments, which allows lookups in logarithmic time. This has been further improved to amortized constant time lookups by means of the hash table data structure (Schrijvers 2005).

A hash table is essentially an array, where at each array location, a list of entries (in our case: suspension terms) is stored. For all operations (lookup, insert, remove), it first computes a hash value for the given key (in our case: the constraint argument on which we are indexing). The hash value determines in which array location the entry is stored. If the hash function is chosen well, the amortized time complexity of all three constraint store operations is expected ${ }^{1}$ constant time.

There can be multiple look-up patterns for the same constraint, so for one constraint, multiple hash tables may need to be maintained.

Example 23 (multiple single-argument hash tables). Say we have a database of telephone numbers encoded as telnum $/ 2$ constraints: telnum(jon,27666) means that jon has telephone number 27666. Consider the following rules:

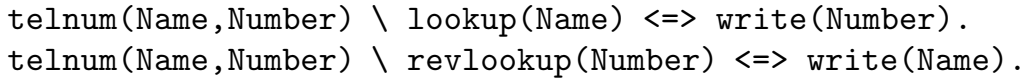

For an active lookup(X) constraint, we need to find telnum/2 constraints where the first $\operatorname{argument}$ is $\mathrm{X}$; for an active revlookup $(\mathrm{Y})$ constraint, we search telnum $/ 2$ constraints with $\mathrm{Y}$ as second argument. In this example, we maintain two hash tables for the telnum/2 constraint because we do lookups on each constraint argument.

Sometimes we need hash tables that index on multiple arguments at once.

Example 24 (multi-argument hash table). Consider the following rule:

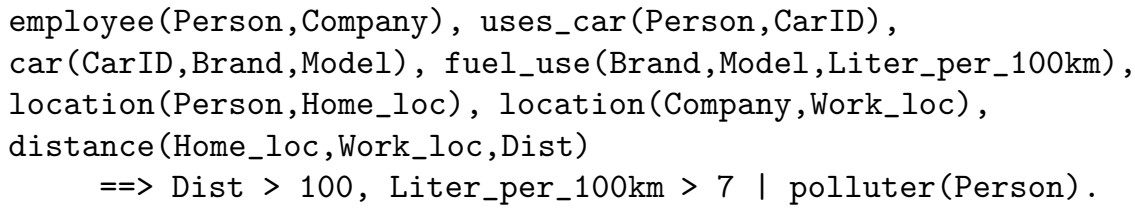

\footnotetext{
${ }^{1}$ If the keys are maliciously chosen, for example such that they all hash to the same value, the lookup time can be as bad as linear. Hence the word "expected", which indicates that we consider the average-case complexity w.r.t. the input keys.
} 
Assume employee $/ 2$ is the active constraint and partner constraints are looked up in the order in which they appear in the rule. For uses_car $/ 2$ and car $/ 3$ hash tables have to be maintained on the first argument. When fuel_use/3 is looked up, both the first and the second argument are known. Hence a multi-argument hash table has to be maintained, which, given a Brand-Model pair, returns all fuel_use/3 constraints with those first arguments. Similarly, for distance/3, a hash table on the first two arguments has to be maintained.

In general, for a constraint with $n$ arguments, up to $2^{n}-1$ hash tables may need to be maintained: one for every subset of arguments (except the empty subset).

(2) The granularity of the hash table based indexes is at the argument level. In other words, for efficient constraint lookups, one or more arguments have to be (fully) known. We do not consider indexing at the sub-argument level. For example, we cannot use the above data structure for lookups via partially instantiated arguments, as in the following rule (where $a / 2$ is the active constraint):

$$
\mathrm{a}(\mathrm{X}, \mathrm{Y}), \mathrm{b}\left(\mathrm{f}\left(_{-}, \mathrm{X}, \mathrm{Z}\right), \mathrm{Y}\right)==>\operatorname{write}(\mathrm{Z}) \text {. }
$$

Sarna-Starosta and Schrijvers (2008) have proposed flattening techniques that bring such sub-argument level matchings to the argument or constraint functor level. In Section 6.4.1 we have discussed an example that illustrates these techniques in the context of the entailment checking program in the implementation of the guard reasoning optimizations.

\subsubsection{Non-ground hash tables}

Clearly, a hash function needs to be deterministic, in the sense that it always returns the same result for a given key. This is also the reason why we demanded the key argument(s) to be ground. A non-ground argument can be further instantiated, and the more instantiated argument could hash to a different value unless of course the hash function maps all (or most) keys to the same hash value, which defeats the purpose of a good hash function.

Hash tables can be extended to support non-ground terms by using attributed variables. Each variable is associated with a unique identifier number, which is used while computing the hash value of a term containing the variable. When the variable is unified to another variable, only one identifier remains, so all hash table entries of which the hash value was based on the other identifier have to be moved. Similarly, when the variable is instantiated, the corresponding entries have to recompute their hash and be moved to the correct location. These relocations cause some overhead at unifications, but the overhead is nearly always negligible compared to the cost of reactivating the constraints that were triggered by the unification.

Using this mechanism, we can support constraint insertion and deletion in $O(1)$ amortized expected time, $O(1)$ lookup of all constraints with a given key, regardless of whether the key is a variable, ground, or a partially instantiated term. 
(1) Non-ground hash tables are currently implemented in the $\mathrm{CHR}^{\mathrm{rp}}$ system by De Koninck et al. (2008). The Leuven JCHR system by Van Weert et al. (2005) contains a Java implementation of logical variables and also supports (non-ground) hash tables. The Leuven CHR system currently only supports ground hash tables.

\subsection{Arrays}

Although under reasonable assumptions, hash table lookups take constant time and insertion and removal operations take constant amortized time, the constant factors are relatively large. In particular, because different keys may hash to the same value, we have to make sure that at a given position in the hash table, multiple buckets can be stored (in case of a collision), and we have to store the key of every bucket and find the bucket with the right key. With a large enough hash table and good hashing functions, the number of collisions can be kept small, but they cannot be completely avoided.

If a constraint argument is not an arbitrary term, but a positive integer number, the constant factors can be improved by using simple arrays instead of hash tables. The integer arguments can be directly used as array positions, avoiding collisions and the need for hash value computations.

We have implemented an additional built-in type, called dense_int. Lookups on ground arguments of this type are implemented as array lookups. Array-based indexes were first introduced in (Sneyers, Schrijvers, and Demoen 2006a). The space usage of an array constraint store is not optimal if the array is sparse, hence the name of the type: it is intended for storing $\Omega(k)$ (asymptotic lower bound) constraints whose argument of type dense_int has values in $[0, k]$.

\subsection{Experimental evaluation}

The current version of the Leuven CHR system supports indexes based on attributed variables, ground hash tables and arrays. Hash tables are only used for arguments that are declared to be ground and arrays are only used for arguments that are declared to be ground and of the type dense_int. Hence, the performance of the Leuven CHR system depends strongly on the amount of detail in the constraint declarations provided by the programmer (see Section 3.5.1).

Consider again the union-find algorithm of Listing 5.2 (page 77). Table 7.1 shows three levels of detail in the constraint declarations for this program. Level 2 corresponds to precise type information, level 1 corresponds to coarser groundness information, level 0 gives no information at all about the constraint arguments.

Figure 7.1 shows the runtime of executing the union-find program in the Leuven CHR system in SWI-Prolog (version 5.6.50). The benchmark consists performing $1000 n$ make/ 1 operations, followed by $1000 n$ random union/2 operations and 


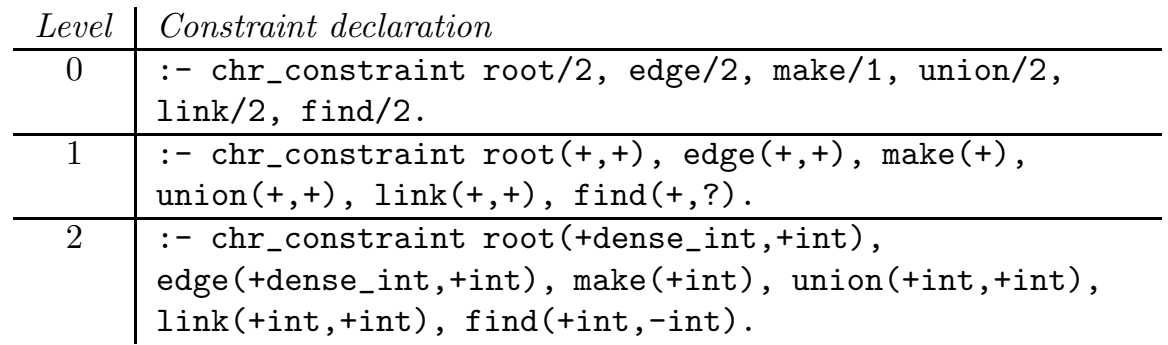

Table 7.1: Different detail levels in mode/type declarations

$1000 \mathrm{n}$ random find $/ 2$ operations. In an optimal implementation of the union-find algorithm, this benchmark should take $O(n \alpha(n))$ time, which can be considered to be linear for all practical purposes ${ }^{2}$. When using hash tables or arrays, this time complexity is indeed achieved, but using the naive data structure (a simple list), the performance degrades to quadratic.

Figure 7.2 shows the same information as Figure 7.1, but this time on logarithmic scales instead of the usual linear scales. Using logarithmic scales has some advantages: for example, a much larger range of input sizes and runtimes can be shown. It is also somewhat easier to see whether two curves have the same asymptotic complexity: if curves tend to be parallel (especially for larger input sizes), they differ only in a constant factor. All polynomial curves are straight lines on a $\log$-log plot; the slope of the line corresponds to the exponent. When evaluating benchmark results, log-log plots are often more informative, although it may be a little trickier to interpret such plots correctly.

Another useful way to plot benchmark results is demonstrated in Figure 7.3. Instead of plotting the runtimes, we now plot the runtime divided by some reference time complexity - in this example, $O(n)$. If a curve is horizontal, it corresponds to the desired reference complexity. Differences in constant factors can still be read from the graph as the distance between two parallel curves.

\subsection{Other possibilities for indexing}

So far, we have considered only indexes for efficient lookups that take implicit (syntactic) equality guards into account. Other types of indexes could be conceived, for example search trees for (arithmetic) inequality guards $(<,>, \leq, \geq$ ). Also, some common patterns of arithmetic equality guards could be supported. We give two examples:

\footnotetext{
${ }^{2}$ The inverse Ackermann function grows very slowly: $\alpha(n)$ is smaller than 5 for $n<2^{2^{10^{19729}}}$ The number of atoms in the observable universe has been estimated to be around $10^{80}$.
} 


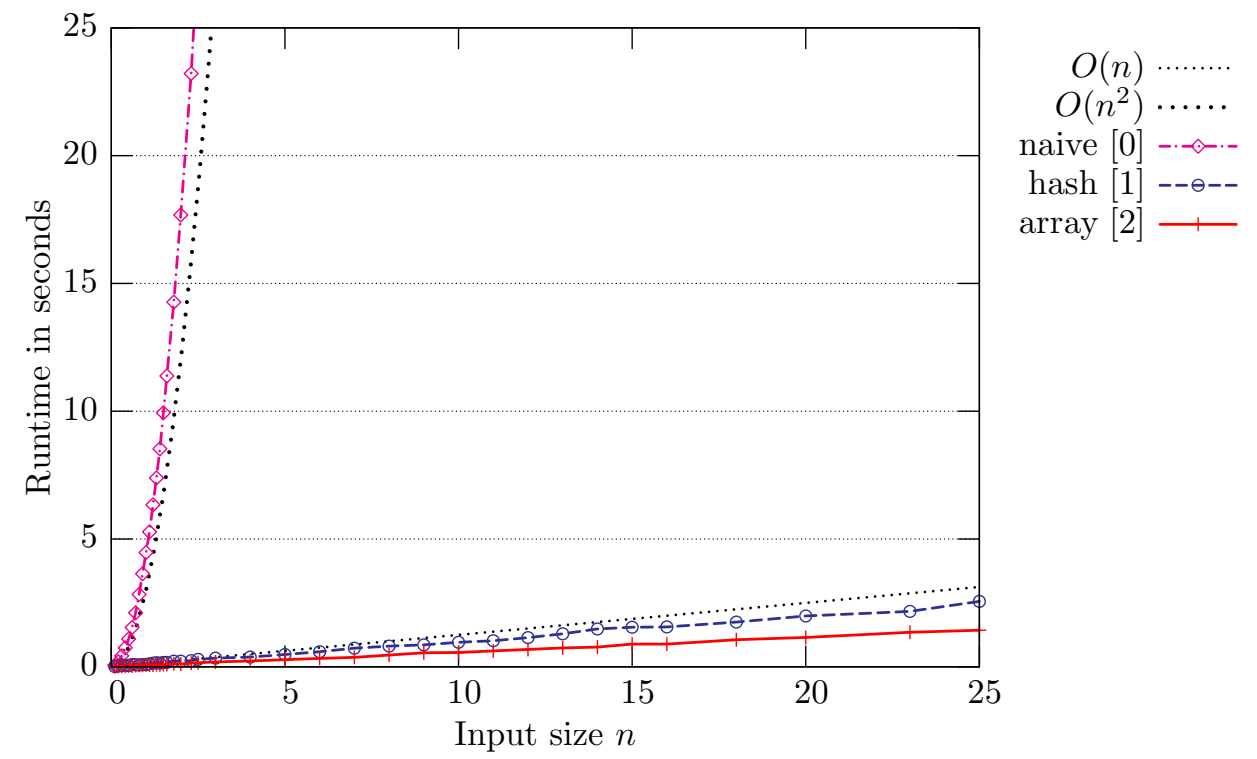

Figure 7.1: Runtimes for the union-find algorithm, with different indexes

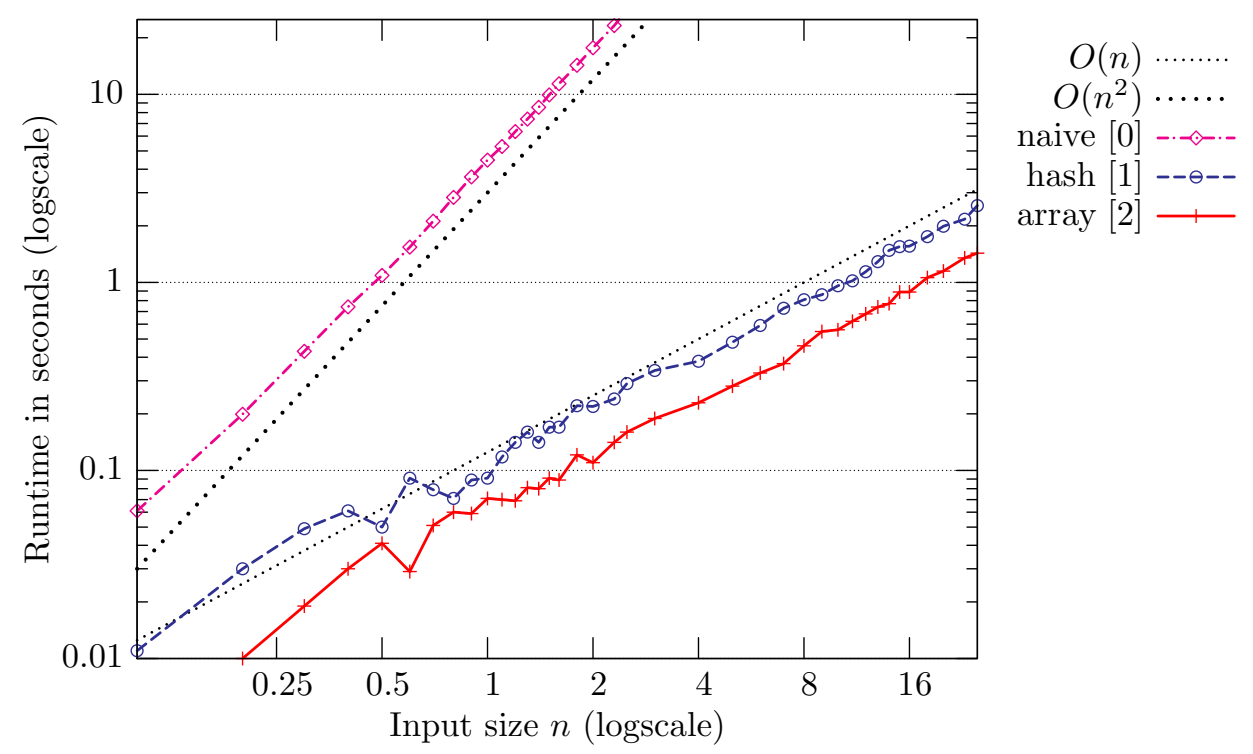

Figure 7.2: The data of the previous plot, on log-log scales 

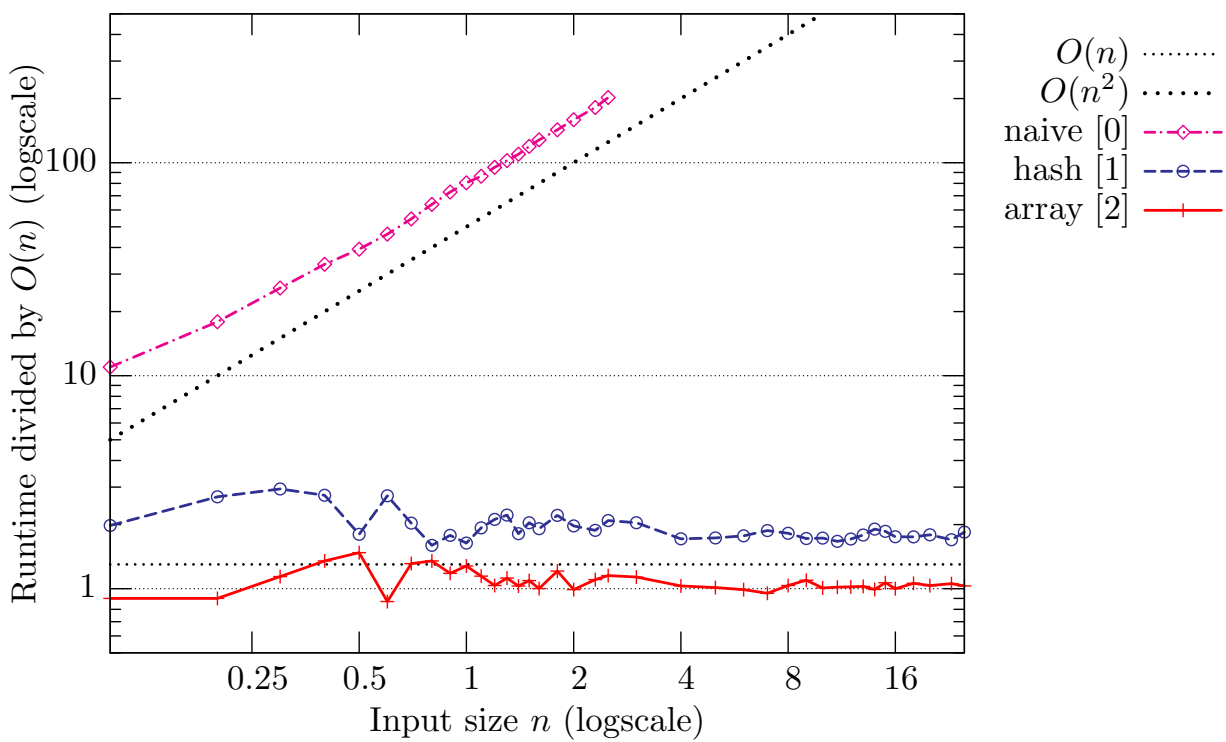

Figure 7.3: The same data, with runtime divided by reference complexity

Example 25 (guard with unique solution). Consider the following rule:

$$
\operatorname{fib}(A, X), f i b(A 1, Y)=\Rightarrow A 1=:=A+1 \mid f i b(A+2, X+Y) \text {. }
$$

If either of the head constraints is active, the guard can be used to uniquely determine the first argument of the partner constraint. Hence the indexing techniques described above (for example a hash table or array) can be used.

Example 26 (guard with multiple solutions). Consider the last rule of the PRIMES program of Listing 3.2 (page 31 ):

absorb o prime $(A) \backslash \operatorname{prime}(B) \Leftrightarrow B \bmod A=:=0 \mid$ true.

Assume the first head constraint, prime (A), is active. The guard does not uniquely determine the value of $B$, but we can do better than iterating over all candidate prime/ 1 constraints. Since B is an integer multiple of A, we can use an array index and lookup only the multiples of A within the bounds of the array.

(0) The term guard solving has been coined for approaches like the above. As far as we know, most CHR systems do not support this, or only to a rather limited extent i.e. only for a few simple and common cases, like the one in Example 25. 


\title{
Chapter 8
}

\section{Memory Reuse}

\author{
"Hegel remarks somewhere that history tends to repeat itself. He \\ forgot to add: the first time as tragedy, the second time as farce." \\ "The philosophers have only interpreted the world, in various ways. \\ The point, however, is to change it."
}

— Karl Marx (1818-1883)

In this chapter we introduce two compiler optimizations that drastically reduce the memory footprint of CHR programs. The reduction is obtained by reusing suspension terms (see Section 4.2.1). The optimizations are defined formally and their correctness is proved. Both optimizations were implemented in the Leuven CHR system. Significant memory savings and speedups were measured.

Section 8.1 describes the space usage issue and informally explains the optimizations that tackle it. In Section 8.2 we present an abstract formal framework that captures the essence of both optimizations, and we prove their correctness. Section 8.3 discusses implementation choices. Experimental results are presented and explained in Section 8.4 and we conclude in Section 8.5.

\subsection{Examples and basic ideas}

Repeatedly replacing a constraint with a new one is a typical pattern that, in current CHR implementations, does not have the space complexity one might expect. The extra space can be reclaimed using (host language) garbage collection, but this comes at a cost in execution time. Indeed, CHR programmers often see that more than half of the total runtime is spent on garbage collection.

The following rules, to be executed in the refined operational semantics, implement a frequently used programming pattern for 'imperative variables' which can be updated destructively: 


\section{Example 27 (imperative variables).}

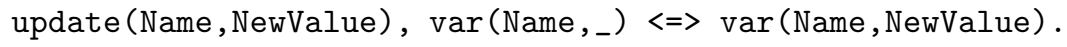

When in this example an update/2 constraint is activated and the update $/ 2$ constraint finds the corresponding 'old' var/2 constraint, internally the following happens: first, the constraint suspension for var/2 is marked 'removed' and it is removed from the constraint store (the suspension becomes garbage), and then a new suspension for the 'new' var/2 constraint is constructed and the 'new' constraint is inserted into the constraint store.

In the above example, the space complexity of performing $n$ update operations on one item is not constant as one might hope for, but $O(n)$. The constraint store overhead and the construction of a new suspension can be avoided by performing the updates in-place. This results in a speedup and a smaller memory footprint. The above program uses only $O(1)$ space when the updates are performed in-place.

\subsubsection{In-place updates}

Rules representing updates have the following form:

$$
C_{1} \backslash C_{2}, c(\bar{a}), C_{3} \quad \Longleftrightarrow \quad G \quad \mid \quad B_{1}, c(\bar{b}), B_{2} .
$$

When the rule is applied, we do not need to remove $c(\bar{a})$ from the constraint store and insert $c(\bar{b})$ into the constraint store. Instead, we can do a direct update on the suspension representing $c(\bar{a})$, updating the constraint arguments in-place.

However, we have to be careful if there are indexes on some argument position(s) for efficient lookups (cf. Chapter 7). If all indexed argument positions are the same in $\bar{a}$ and $\bar{b}$, we can avoid all constraint store overhead. Otherwise we have to remove and reinsert the constraint from the indexes on modified argument positions. In many cases, a constraint is indexed on more than one argument, i.e. when different lookup patterns are used. We have to do the remove/insert only on the affected indexes - which is still better than having to do a full remove/insert.

Example 28 (affected indexes). Consider the following rule:

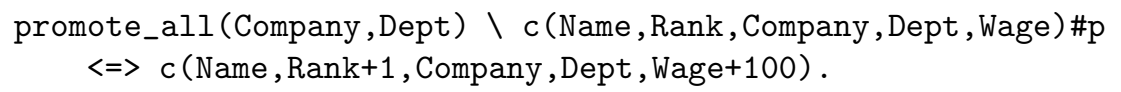

Assume that for the $\mathrm{c} / 5$ constraints, indexes are maintained on argument positions 1 (Name) and 5 (Wage). For this rule an additional index on the combined argument positions 3-4 is needed. Figures 8.1 and 8.2 show what happens if the above rule is applied, without and with in-place updates, respectively. 


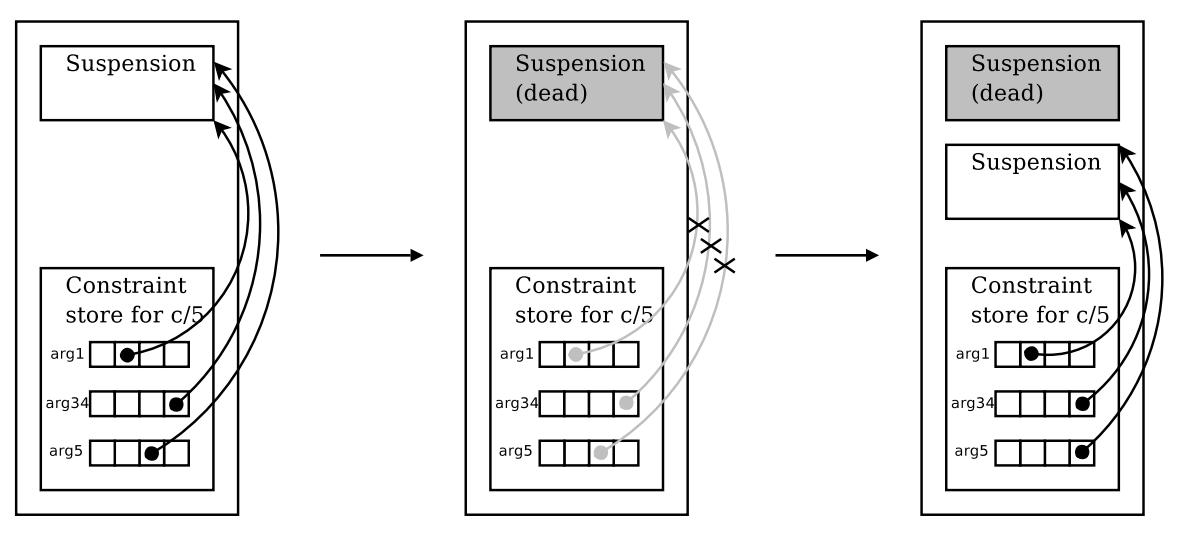

Figure 8.1: Constraint deletion followed by insertion, without optimizations

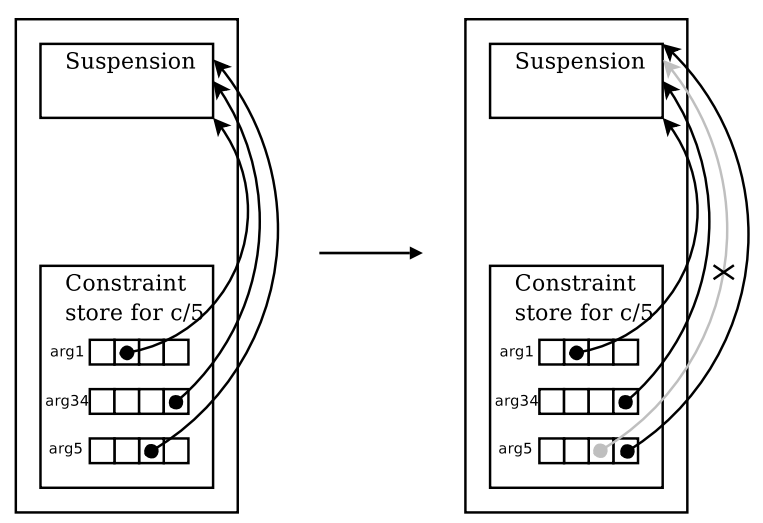

Figure 8.2: In-place update 
In many programs, the updates are not direct, as in Example 27, but rather indirect and perhaps only partial, like in this example:

Example 29 (indirect updates). Consider again the PRIMES program:

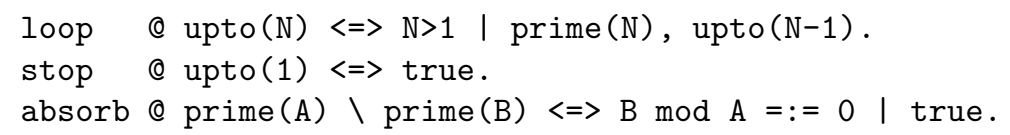

In this program, the in-place update optimization is ineffective, since there is no direct update: the third rule removes prime/ 1 constraints while the first rule inserts prime/1 constraints.

In general there can be an arbitrary amount of intermediate computations between the removal and the insertion. To tackle such indirect updates, we propose a generalization of the in-place update optimization, called suspension reuse.

\subsubsection{Suspension reuse}

The suspension reuse optimization avoids constructing a new suspension term by maintaining a cache of old suspensions which can be reused. The time and space gains are typically smaller than in the in-place update optimization, but suspension reuse has a wider applicability. It works as follows:

When a constraint is removed, its suspension is added to the cache, but it is not (yet) removed from the constraint store. The suspension is marked so it can be skipped when found later in a partner constraint lookup.

When a new constraint is added and a suspension has to be created, an old suspension is extracted from the cache and its arguments are updated to those of the new constraint. A remove/insert is done only on the indexes that are affected by modified arguments, saving some (or all) constraint-store overhead. Only when the cache is empty, a new term has to be created and a full insert has to be done. Note that it makes sense to restrict the maximum size of the cache. The mechanism is illustrated schematically in Figure 8.3 for the rule of Example 28.

\subsection{Formal framework}

In this section we extend the operational semantics of CHR to capture the reuse of removed constraints (section 8.2.1). By showing that this extension is equivalent to the original semantics (section 8.2.2), and by formulation our optimizations as particular instances of the extension (section 8.2.3), correctness follows.

We start from the call-based refined operational semantics $\omega_{c}$, as defined in Section 4.3.3 (see Figure 4.4 on page 70 ). In addition to the original transitions of $\omega_{c}$, we also allow the following transition, which removes redundant history tuples: 


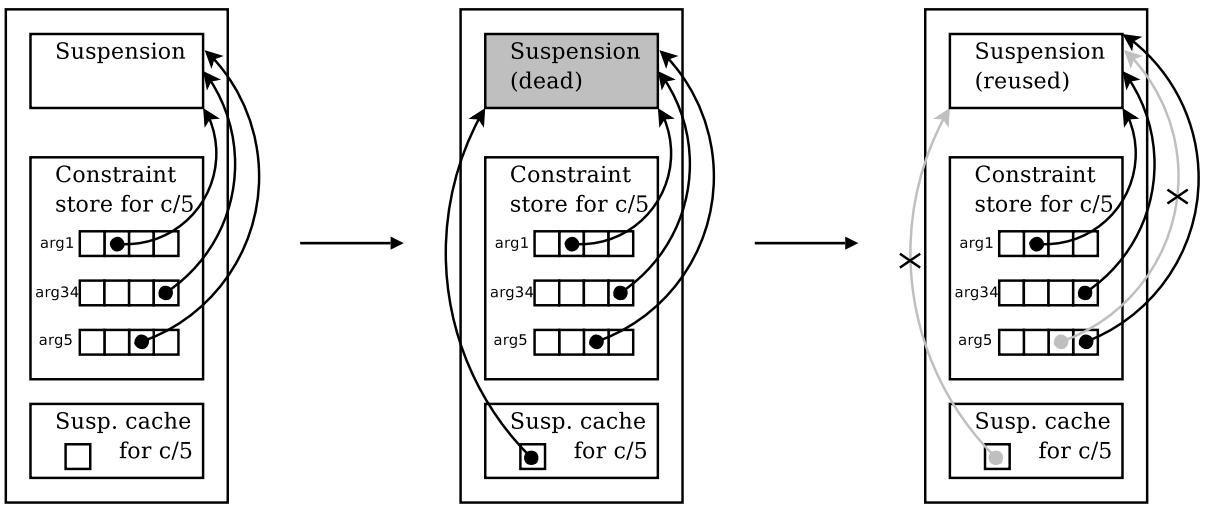

Figure 8.3: Constraint deletion followed by insertion, with suspension reuse

7. CleanHistory. $\langle\mathbb{G}, \mathbb{A}, \mathbb{S}, \mathbb{B}, \mathbb{T} \cup\{t\}\rangle_{n} \succ_{\omega_{c}, \mathcal{P}}\langle\mathbb{G}, \mathbb{A}, \mathbb{S}, \mathbb{B}, \mathbb{T}\rangle_{n}$ if $t$ contains an identifier which is not in $\mathbb{S}$, i.e. $i d(t) \backslash i d(\mathbb{S}) \neq \emptyset$.

Applying this transition clearly does not affect a derivation. The CleanHistory transition only removes a redundant tuple of the propagation history redundant in the sense that it will never block a rule application attempt, since the constraints it refers to are no longer in the constraint store.

The execution strategy implemented in the Leuven CHR system is an instance of the above semantics (and not of the original formulation, which keeps all propagation history tuples, forever). In the Leuven CHR system (and probably in all CHR systems), the propagation history is only checked and updated when applying a propagation rule. Simplification and simpagation rules cannot be applied twice to the exact same constraints, since at least one of the head constraints is removed after the first application. We can simulate this behavior by applying the CleanHistory transition after firing a non-propagation rule. Also, the Leuven CHR system maintains the propagation history in a distributed way: suspensions contain history tuples added when the constraint they represent was active (see Section 4.4.1). As a result, when a constraint is removed and its suspension becomes garbage, the corresponding tuples are automatically removed from the propagation history. This corresponds to applying CleanHistory a number of times.

(1) The Leuven CHR system only removes a history tuple (by means of Prolog garbage collection) when the constraint is removed that was the active constraint when the rule was applied that added the tuple. The tuple may already be removed as soon as any constraint in it is removed. In other words, the Leuven CHR system does not always apply the CleanHistory transition even if it is applicable. 
2c. Reuse. $\left\langle c, \mathbb{A},\left\{\dagger c^{\prime} \# i\right\} \uplus \mathbb{S}, \mathbb{B}, \mathbb{T}\right\rangle_{n} \mapsto_{\omega_{c}^{\prime}, \mathcal{P}}\langle c \# n: 1, \mathbb{A},\{c \# n\} \uplus \mathbb{S}, \mathbb{B}, \mathbb{T}\rangle_{n+1}$ where $c$ is a CHR constraint which has never been active.

2d. ReuseID. $\left\langle c, \mathbb{A},\left\{\dagger c^{\prime} \# i\right\} \uplus \mathbb{S}, \mathbb{B}, \mathbb{T}\right\rangle_{n} \longmapsto_{\omega_{c}^{\prime}}, \mathcal{P}\langle c \# i: 1, \mathbb{A},\{c \# i\} \uplus \mathbb{S}, \mathbb{B}, \mathbb{T}\rangle_{n}$ where $c$ is a CHR constraint which has never been active and $\forall t \in \mathbb{T}: i \notin t$.

4b. Simplify'. $\left\langle c \# i: j, \mathbb{A},\{c \# i\} \uplus H_{1} \uplus H_{2} \uplus H_{3} \uplus \mathbb{S}, \mathbb{B}, \mathbb{T}\right\rangle_{n} \hookrightarrow_{\omega_{c}^{\prime}, \mathcal{P}}\left\langle\square, \mathbb{A}, \mathbb{S}^{\prime}, \mathbb{B}^{\prime}, \mathbb{T}^{\prime \prime}\right\rangle_{n^{\prime}}$ with $\left\langle\theta(C), \mathbb{A}, H_{1} \uplus \dagger\left(\{c \# i\} \uplus H_{1} \uplus H_{2}\right) \uplus \mathbb{S}, \theta \wedge \mathbb{B}, \mathbb{T}^{\prime}\right\rangle_{n} \longmapsto \omega_{c}^{\prime}, \mathcal{P}\left\langle\square, \mathbb{A}, \mathbb{S}^{\prime}, \mathbb{B}^{\prime}, \mathbb{T}^{\prime \prime}\right\rangle_{n^{\prime}}$ and with the other conditions as in the usual Simplify transition.

5b. Propagate' . Defined exactly as the usual Propagate transition, except that the series of subderivations is now

$$
\begin{aligned}
& \left\langle C_{l},[c \# i: j \mid \mathbb{A}], H_{1 l} \uplus\{c \# i\} \uplus H_{2 l} \uplus \dagger H_{3 l} \uplus R_{l}, \mathbb{B}_{l-1}, \mathbb{T}_{l-1} \cup\left\{t_{l}\right\}\right\rangle_{n_{l-1}} \\
& \longmapsto \omega_{c}^{\prime}, \mathcal{P}\left\langle\square,[c \# i: j \mid \mathbb{A}], \mathbb{S}_{l}, \mathbb{B}_{l}, \mathbb{T}_{l}\right\rangle_{n_{l}}
\end{aligned}
$$

8. RemoveDead. $\langle\mathbb{G}, \mathbb{A},\{\dagger c \# i\} \uplus \mathbb{S}, \mathbb{B}, \mathbb{T}\rangle_{n} \longmapsto_{\omega_{c}^{\prime}, \mathcal{P}}\langle\mathbb{G}, \mathbb{A}, \mathbb{S}, \mathbb{B}, \mathbb{T}\rangle_{n}$

Figure 8.4: Additional transitions of the extended call-based semantics $\omega_{c}^{\prime}$

\subsubsection{Extended call-based refined operational semantics $\omega_{c}^{\prime}$}

We now extend the $\omega_{c}$ semantics by allowing the constraint store to contain dead constraints, which are removed and cannot be used to match the head constraints of a rule. These constraints form the cache from which the suspension reuse optimization recycles suspension terms. (In case of the in-place update optimization, the cache is implicit.) A dead constraint $c$ with identifier $i$ is denoted as $\dagger c \# i$. When $X$ is a set of (non-dead) constraints, we write $\dagger X$ to denote $\{\nmid x \mid x \in X\}$. The extended call-based refined operational semantics $\omega_{c}^{\prime}$ is obtained by extending $\omega_{c}$ with the transitions listed in Figure 8.4.

The Reuse transition and the ReuseID transition are variants of the Activate transition which replace a dead constraint by a newly added constraint. While the Activate and Reuse transitions assign a new identifier, the ReuseID transition reuses the identifier of the dead constraint. The Simplify ${ }^{\prime}$ and Propagate ${ }^{\prime}$ transitions are variants of the corresponding $\omega_{c}$ transitions that mark the removed constraints as being dead instead of completely deleting them from the store. Finally, the RemoveDead transition deletes a dead constraint.

\subsubsection{Equivalence of $\omega_{c}$ and $\omega_{c}^{\prime}$}

It is clear that every $\omega_{c}$ derivation is also a valid $\omega_{c}^{\prime}$ derivation, since $\omega_{c}^{\prime}$ is an extension of $\omega_{c}$. We now show that every $\omega_{c}^{\prime}$ derivation (denoted with $\hookrightarrow_{\omega_{c}^{\prime}}, \mathcal{P}$ ) can be mapped to an $\omega_{c}$ derivation (denoted with $\mapsto_{\omega_{c}, \mathcal{P}}$ ). 
Definition 8.1 (visible part) Given an $\omega_{c}^{\prime}$ execution state $\sigma$ which is of the form $\left\langle\mathbb{G}, \mathbb{A}, \mathbb{S}_{l} \uplus \dagger \mathbb{S}_{d}, \mathbb{B}, \mathbb{T}\right\rangle_{n}$, where $S_{l}$ contains no dead constraints, the visible part of $\sigma$ is $\Psi(\sigma)=\left\langle\operatorname{chr}\left(\mathbb{S}_{l}\right), \mathbb{B}\right\rangle$.

Definition 8.2 (indistinguishable states) Two execution states $\sigma_{1}$ and $\sigma_{2}$ are indistinguishable if and only if $\Psi\left(\sigma_{1}\right)=\Psi\left(\sigma_{2}\right)$. We denote this by $\sigma_{1} \cong \sigma_{2}$.

Theorem 8.3 Suppose $\sigma_{0}$ is an initial execution state. If $\sigma_{0} \longmapsto_{\omega_{c}^{\prime}, \mathcal{P}}^{*} \sigma_{1}$, then an execution state $\sigma_{2}$ exists such that $\sigma_{0} \longmapsto \omega_{c}, \mathcal{P} \sigma_{2}$ and $\sigma_{1} \cong \sigma_{2}$.

Proof. If the ReuseID transition is not used in the $\omega_{c}^{\prime}$ derivation, we can derive $\sigma_{2}$ from $\sigma_{0}$ in $\omega_{c}$ by replacing every Reuse by a corresponding Activate, Simplify ${ }^{\prime}$ by a corresponding Simplify, Propagate' ${ }^{\prime}$ by a corresponding Propagate and removing all RemoveDead transitions from the original $\sigma_{0} \longmapsto \omega_{c}^{\prime}, \mathcal{P} \sigma_{1}$ derivation. The only difference between $\sigma_{1}$ and $\sigma_{2}$ is in their CHR stores $\mathbb{S}_{1}$ and $\mathbb{S}_{2}$ : it is easy to see that $\mathbb{S}_{2} \subseteq \mathbb{S}_{1}$ and that $\mathbb{S}_{1} \backslash \mathbb{S}_{2}$ contains only dead constraints. Hence the result trivially holds in this case.

If the ReuseID transition is used $r$ times, we compute an identifier permutation $\mu=\mu_{r}$ as follows. Let $\mu_{0}$ be the identical permutation. We consider every ReuseID transition in the $\omega_{c}^{\prime}$ derivation from $\sigma_{0}$ to $\sigma_{1}$, in order. Assume the $k$-th of these ReuseID transitions is the following:

$$
\left\langle c, \mathbb{A},\left\{\dagger c^{\prime} \# i\right\} \uplus \mathbb{S}, \mathbb{B}, \mathbb{T}\right\rangle_{n} \longmapsto \omega_{c}^{\prime}, \mathcal{P}\langle c \# i: 1, \mathbb{A},\{c \# i\} \uplus \mathbb{S}, \mathbb{B}, \mathbb{T}\rangle_{n}
$$

Let $\mu_{k}(m)=\mu_{k-1}(m)$ for all $m<n$ and $m \neq i, \mu_{k}(i)=\mu_{k-1}(n)$, and $\mu_{k}(m)=$ $\mu_{k-1}(m+1)$ for all $m \geq n$. Since the reused identifier $i$ does not occur in the propagation history (by definition of the transition), it will uniquely identify the activated constraint just like the fresh identifier $n$. Hence, replacing the ReuseID transition by a Reuse transition does not affect the rest of the derivation. By applying the previous case on $\sigma_{0} \longmapsto_{\omega_{c}^{\prime}, \mathcal{P}}^{*} \mu\left(\sigma_{1}\right)$, we get a $\sigma_{2}$ such that $\sigma_{0} \succ_{\omega_{c}, \mathcal{P}}^{*} \sigma_{2}$ and $\sigma_{2} \cong \mu\left(\sigma_{1}\right)$. From $\sigma_{2} \cong \mu\left(\sigma_{1}\right)$ and $\mu\left(\sigma_{1}\right) \cong \sigma_{1}$ (identifier permutations clearly do not affect the visible part) we can conclude $\sigma_{1} \cong \sigma_{2}$.

\subsubsection{Defining the optimizations}

Definition 8.4 (suspension reuse) Suspension reuse with maximum cache size $n$ corresponds to the following $\omega_{c}^{\prime}$ execution strategy: always perform the Simplify' and Propagate' transitions (instead of Simplify and Propagate), followed by RemoveDead transitions until there are no more than $n$ dead constraints with the same predicate name in the store. Furthermore, the Activate transition is only applied when Reuse or ReuseID are not applicable. 


\begin{tabular}{c|c|c}
\hline State & In implemented store? & In theoretical store? \\
\hline stored & yes & yes \\
removed & no & no \\
not_stored & no & yes \\
limbo & yes & no \\
\hline
\end{tabular}

Table 8.1: Meaning of the MState field in constraint suspensions

Definition 8.5 (in-place updates) Performing an updates in-place corresponds to the following $\omega_{c}^{\prime}$ execution strategy: if the goal is an occurrenced constraint and the corresponding rule is of the form " $C_{1} \backslash C_{2}, c(\bar{a}), C_{3} \Longleftrightarrow G \mid B_{1}, c(\bar{b}), B_{2}$." (as in Section 8.1.1), then perform the Simplify' and Propagate' $^{\prime}$ transitions (not the Simplify and Propagate transitions). In the subderivation for the rule body (assuming the rule fired), the removed constraint $c(\bar{a})$ is reused in a Reuse or ReuseID transition when the goal is $c(\bar{b})$.

Both optimizations are defined as an execution strategy which instantiates the $\omega_{c}^{\prime}$ semantics. Because of Theorem 8.3, this implies that the optimizations are correct with respect to the visible part of CHR.

\subsection{Implementation of memory reuse}

In this section we describe our implementation of the new optimizations in the Leuven CHR system.

\subsubsection{Constraint representation}

Recall that we use the following internal representation for a constraint:

suspension (ID, MState, Continuation, MHistory, $\mathrm{C}, \mathrm{X}_{1}, \ldots, \mathrm{X}_{n}$ )

The meaning of the fields is listed in Table 4.1 on page 64 .

For the purpose of the suspension reuse optimization, we add the value "limbo" to the list of possible values for the MState field (in addition to stored, removed, and not_stored). The limbo value means that the constraint is removed, but still in the store. We will not immediately need this new limbo state, but we already discuss it now in order to remind the reader of the suspension representation details discussed earlier in Section 4.2.1.

If we distinguish between the implemented store (the data structure(s) representing the constraint store) and the theoretical store (the constraint store as it would be in the corresponding $\omega_{r}$ or $\omega_{c}$ derivation), possible states for a constraint suspension can be described as in Table 8.1. 


\subsubsection{Inlining and specialization}

We can improve performance of the generated code by inlining calls to the auxiliary predicate insert_constraint_internal (see Section 4.2.2). As a result, it is no longer necessary to wrap the constraint arguments in a list, and the generic term creation using $=$. . can be replaced by unification with a term with an arity which is known at (Prolog) compile-time. This specialization saves some time and space, especially for constraints with many arguments.

Similarly, we can also inline and specialize the calls to auxiliary predicates for constraint removal and constraint lookups.

\subsubsection{Suspension reuse}

In our implementation we use cache size 1 (per CHR constraint predicate). This allows easy and efficient cache manipulation and uses little extra space. For every constraint predicate we maintain a backtrackable global variable, which is initialized to an atom representing an empty cache. When a constraint is removed, the variable is checked: if it is empty, it is overwritten with the suspension term; otherwise, the usual remove operation is performed. In both cases, the suspension state is set to removed. When a suspension term is constructed for some constraint, the global variable is checked. If it is empty, the usual term construction is done we call this a cache miss. Otherwise the suspension term in the cache is reused and the global variable is updated to the empty atom — this is called a cache hit.

To reuse a suspension term, the fields for the constraint arguments must be updated. The constraint functor field can be left untouched since we have a different cache for every distinct CHR constraint predicate. The other fields may need to be overwritten. We use the backtrackable hProlog built-in setarg/3 (modifies one argument of a term) to update suspension term fields and b_setval/2 (modifies a global variable) to implement the cache. Although CHR rules are committedchoice, choice-points can be left by host language predicates that are called in the rule bodies or that call CHR constraints, so it is important to use backtrackable versions of those destructive update built-ins.

\section{Fields to overwrite}

Note that in general it requires an inspection of the entire constraint store which is very expensive - to check whether keeping the old identifier is safe. For this reason our implementation uses the following approximation: If the constraint occurs in a multi-headed propagation rule with at least one (other) active head, the identifier field is always overwritten with a fresh identifier (this corresponds to applying the Reuse transition). Otherwise, the propagation history tuples in which the old identifier occurs are all stored in the cached (dead) suspension. In that case we overwrite the suspension term field storing the partial history 
with a term representing an empty partial history (this corresponds to a series of CleanHistory transitions). We can reuse the old identifier (the ReuseID transition), since it no longer occurs in the propagation history. Of course, if the constraint does not occur in propagation rules at all, the suspension field for the propagation history is not used so it does not need to be overwritten.

One final subtle implementation issue is the following. If the constraint has an active kept occurrence in a rule with a body which might cause the removal and reuse of the active constraint, we have to make sure that we detect this at the return from the body, so we can avoid incorrectly calling the continuation of a removed constraint. If the active constraint is removed, but not reused, it suffices to check the state field before calling the continuation. However, if it is reused after removal, the state field will have reverted, rendering that check ineffective. To solve this issue, we also check after executing the body whether the constraint identifier is unmodified. To make sure the test is effective, we never use the ReuseID transition for such constraints.

\subsubsection{In-place updates}

If all occurrences of the in-place updated constraint are passive, it suffices to update the suspension term as above and update the affected parts of the constraint store. The potentially modified arguments are determined statically by comparing the arguments of the removed head constraint and the arguments of the constraint call in the body. For every potentially affected index, we test at run-time whether the index position has changed and if it has, we do a remove and reinsert in that index. If the part of the body before the insertion $\left(B_{1}\right.$ in Section 8.1.1) does not observe $^{1}$ the removal of the updated constraint (e.g. if it is empty or a conjunction of safe host language built-ins), then we do not need to set the MState field of the suspension to removed before executing $B_{1}$ and restore it to stored afterwards.

If there are non-passive occurrences of the updated constraint, we additionally have to call the first occurrence sub-procedure after performing the update.

(1) There is a choice in how to do the updates if there is more than one removed head constraints or more than one body constraint with the same functor and arity. In our current implementation we simply match the $i$-th removed constraint with the $i$-th inserted constraint. This is a simple but suboptimal strategy. Consider the rule " $\mathrm{a}(\mathrm{A}, \mathrm{C}) \backslash \mathrm{b}(\mathrm{A}, \mathrm{B}), \mathrm{b}(\mathrm{C}, \mathrm{D}) \Leftrightarrow \mathrm{b}(\mathrm{C}, \mathrm{C}), \mathrm{b}(\mathrm{A}, \mathrm{A})$." where the constraint $b / 2$ is indexed on its first argument. If $b(A, B)$ is updated to $b(A, A)$ and $b(C, D)$ is updated to $b(C, C)$, no index is affected and at most two arguments have to be updated, which clearly is optimal. The simple matching strategy has two possibly affected indexes and four arguments to be updated.

\footnotetext{
${ }^{1}$ See Section 4.4.5 for more on observation analysis.
} 


\subsubsection{Interference with late storage}

The late storage optimization (see Section 4.4.2) may delay the creation of a suspension term and the insertion into the constraint store, so overhead is avoided if the constraint is removed before the suspension creation or before the constraint store insertion. Although the late storage optimization clearly has its advantages, the combination with memory reuse techniques causes some problems.

\section{Late storage and suspension reuse}

For the suspension reuse optimization, we want to maintain the invariant that cached suspensions are in the data structure implementing the constraint store. Hence, if a constraint is removed after its suspension term has been created but before it has been inserted into the data structure, we do not put it in the cache.

To cooperate with the late storage optimization, we delay the constraint store update until the late insertion program point. If that point is at a later occurrence than the suspension creation, we need to distinguish between a fresh suspension (which needs a full insert) and a reused suspension (which needs an in-place store update). This is done by adding a new suspension state "limbo", which mostly acts like not_stored, except at insertion and early removal. The constraint arguments in the suspension term are not yet updated at the point of suspension reuse, but the reused suspension's state is set to limbo. At insertion, we still have the old arguments in the suspension term, so we can compare them to the new arguments (which are available as passed arguments in the occurrence sub-procedures) to do a store update (repairing the indexes). If the constraint is removed early, i.e. before the insertion point, we still have to remove the old suspension from the store.

\section{Late storage and in-place updates}

Late storage also interferes with the in-place update optimization. If the suspension is not created before the first occurrence, we can not simply call the predicate for the first occurrence. The reason is that at some point in that subcomputation, a suspension might be created and the constraint might be stored again, which would duplicate the constraint. To solve that problem, we generate additional clauses with a different predicate name. In the additional clauses, the late storage optimization is disabled, preventing a second suspension creation and constraint insertion. Note that adding these clauses can almost double the code size, which may have a negative effect on the runtime.

When an in-place update is performed, the old suspension is updated and the stores are updated. Without in-place updates we would remove the old constraint and call the new one. If the new constraint is removed before it is stored, the overhead of insertion and removal is avoided by late storage. Hence, without inplace updates we only do one full remove in this case. With in-place updates, we 
do an in-place store update (a partial remove and partial insert) followed by a full remove (of the new constraint). As a result, when late storage is involved, there is only a performance gain if the new constraint is not removed early.

\subsection{Experimental results}

We now present an evaluation of the optimizations introduced in the previous sections. Table B.3 lists the results. One clear general conclusion is that we can drastically improve both time and space usage of the generated code by inlining and specializing calls to the auxiliary predicates for suspension term construction, removal, and lookup (see Section 8.3.2). Inlining can cut both time and space in half, and it never worsens either time or space. As a reference for evaluating the memory reuse optimizations, we consider the situation where inlining is enabled. Of course this means it is harder for the optimizations to look good since much of the overhead they try to avoid is already greatly reduced by inlining.

Suspension reuse. Suspension reuse introduces some runtime overhead for maintaining the suspension cache. If the dynamic behavior of the program is such that the cache is (almost) always empty at suspension creation, i.e. if there are few cache hits and many cache misses, we do not get time or memory benefits. Examples like bool_chain and inference show that with few cache hits, the time overhead can be significant: 5 to $10 \%$. Although there are many cache hits in the FIB-HEAP benchmark, the runtime still increases. The reason is that the indexes used in FIB-HEAP (arrays and one singleton constraint) provide very cheap insertion and removal operations. The overhead of maintaining the suspension cache and dynamically checking for affected indexes is simply higher than the cost of the avoided store operations. In the other benchmarks, suspension reuse results in a net gain in both time and space. The speedup is typically between 5 and 25 percent, but can be as high as $59 \%$ (in the PRIMES benchmark). The space reduction is more than $60 \%$ in some benchmarks.

In-place updates. In-place updates are not always applicable: the bool_chain and PRIMES programs do not contain any rule of the required form. However, when they are, the time and space gains are quite impressive. In the RAMSIMUL benchmark, all space usage is eliminated from the main loop, reducing the space complexity from linear to constant. In other examples, space gains are typically between 20 and 30 percent. Speedups are typically between 15 and 40 percent. There are more gains if the updated constraint has no active occurrences and if there are few indexes that are affected by the update. For example, compare the SUDOKU and RAMSIMUL benchmarks to the FIB-HEAP and inference benchmarks. In the former, no indexes are affected and there are no active occurrences, resulting in a $40 \%$ speedup and a $80 \%$ memory reduction (100\% in the case of 
RAMSIMUL). In the latter, there is a small slowdown and only a marginal reduction in memory use. Because of the active occurrences, the effect of the late storage optimization is largely lost. If all indexes are affected, the update that replaces the remove and insert is as expensive as a full remove and insert.

When combining in-place updates and suspension reuse, the number of cache hits drops: many in-place updates 'steal' a hit. As a consequence, the overhead of suspension reuse often outweighs the gains if (many) in-place updates are done. A simple heuristic could be added to avoid this.

The refined operational semantics of CHR (see Section 4.3) leaves the order in which partner constraints are tried unspecified. Hence, programs (even those programs that depend on the refined semantics) should not depend crucially on the order used in some specific CHR system. Both suspension reuse and in-place updates can affect this order.

For some programs, the different partner constraint order does significantly influence the derivations, for example because the order has an impact on the search space. This may result in unexpected differences in performance. An example is the SUDOKU benchmark with a different initial board setup (one with 2 solutions and 16 givens), where in-place updates result in a slowdown of $46 \%$, while suspension reuse results in a speedup of $64 \%$ - both effects are mostly caused by the different order. The new order can in principle be arbitrarily better or worse than the original one. Of all programs listed in Table B.3, only SUDOKU and inference are affected in this way. For the query instances listed in the table, the search space is not significantly changed when the partner constraint order is modified by the optimizations. Sophisticated analyses and/or heuristics could be conceived to improve the order but that is completely outside the scope of this work.

\subsection{Conclusion}

As far as we know, this is the first proposal for techniques specifically aimed at improving the memory footprint of CHR programs. By inlining and specializing some crucial parts of the generated code we already achieved a dramatic improvement in both time and space. However, our main contribution is twofold: we have provided a general formal framework that allows us to formulate and reason about reuse of removed constraints at an abstract level, and we have introduced two new optimizations, in-place updates and suspension reuse, and proved their correctness. We have implemented both optimizations in the Leuven CHR system, which revealed that they interfere with the existing late storage optimization. This interference complicates the implementation and it also decreases the effect of in-place updates. Experimental results indicate that both optimizations can cause small slowdowns in some benchmarks and big speedups in others. The memory footprint is improved in all benchmarks - sometimes only marginally, often significantly. 


\subsubsection{Related work}

This work is somewhat related to compile-time garbage collection (Mazur 2004; Mazur, Ross, Janssens, and Bruynooghe 2001). In-place updates are related to direct structure reuse as defined in Chapter 9 of (Mazur 2004) in the context of Mercury compilation, while suspension term reuse is related to indirect structure reuse. The analysis required for CTGC on arbitrary Mercury (or Prolog) programs is quite involved. Luckily, we only had to consider the particular code of the compilation schema used in the CHR compiler. Hence we could, so to speak, 'manually specialize' the CTGC liveness and reuse analyses to conditions on the CHR program to be compiled.

In the old SICStus CHR system (in SICStus 3), the option already_in_heads is offered, both as a global compiler option and a rule pragma. The effect of this pragma is as follows: when a body constraint is identical to one of the removed head constraints, the removal and reinsert is avoided and the constraint is not activated. This roughly corresponds to the in-place update optimization, restricted to the case where none of the arguments are modified. However, the already_in_heads option may affect the behavior of the CHR program. The CHR programmer is responsible for verifying whether the operational semantics are preserved by the option. Also, it is always possible to avoid the already_in_heads pragma and simulate its effect by strengthening the guard or by adding rules. For example, the following rule:

$\operatorname{diff}(\mathrm{X}, \mathrm{Y}) \backslash \operatorname{dom}(\mathrm{X}, \mathrm{L}) \Leftrightarrow \operatorname{delete}(\mathrm{L}, \mathrm{Y}, \mathrm{NL}), \operatorname{dom}(\mathrm{X}, \mathrm{NL})$ pragma already_in_heads. can be rewritten without the pragma, as follows:

$\operatorname{diff}(\mathrm{X}, \mathrm{Y}) \backslash \operatorname{dom}(\mathrm{X}, \mathrm{L}) \Leftrightarrow \operatorname{delete}(\mathrm{L}, \mathrm{Y}, \mathrm{NL}), \mathrm{L} \backslash==\mathrm{NL} \mid \operatorname{dom}(\mathrm{X}, \mathrm{NL})$.

Another approach is to add rules of the form $c \backslash c \Longleftrightarrow$ true before all other rules, in order to enforce set semantics. The result is that when a duplicate constraint is added, it is immediately removed.

\subsubsection{Future work}

There are many possibilities to improve and extend the current implementation. For example, bigger suspension cache sizes could increase the number of cache hits. Also suspension terms could be reused even if the constraint functor is different (and not only when the arity is the same). In rules with two or more in-place updates we could compute the optimal matching between removed head constraints and inserted body constraints, minimizing the number of affected indexes. It would also be interesting to investigate the impact of modifying the order in which partner constraints are tried, and perhaps mechanisms can be conceived to control and improve that order. Finally, some heuristics could be added to decide whether or not to enable the optimizations. 


\section{Chapter 9}

\section{Join Ordering}

"A means can be justified only by its end. But the end in its turn needs to be justified."

- Leon Trotsky (1879-1940)

A crucial aspect of CHR compilation is finding matching rules efficiently. Given an active constraint, searching for matching partner constraints corresponds to joining relations - a well-studied topic in the context of databases. ${ }^{1}$ The performance of join methods is determined by the efficiency of the indexing techniques and by join ordering. We have discussed indexing in Chapter 7. This chapter deals with the complementary problem of join ordering.

In the context of CHR, join ordering has been investigated by Holzbaur et al. (2005) and by Duck (2005). In that work, only static (compile-time) information is used in determining the optimal join order. Moreover, little attention is given to the complexity of the optimization algorithm, since join ordering is done at compile time and because the input sizes (the number of heads of a rule) are expected to be very small - "usually at most 3 in practice" (Duck 2005). In this chapter we consider dynamic (run-time) join ordering based on constraint store statistics such as selectivities and cardinalities. We also take into account that CHR programs increasingly contain rules with more than three heads. Some examples of such programs are given in Section 9.5.2.

Our main contributions are threefold. Firstly, we formulate a generic cost model for join ordering, which provides a solid foundation to develop and evaluate heuristics. Secondly, we introduce dynamic join ordering. Thirdly, we discuss an efficient $O(n \log n)$ join order optimization algorithm for acyclic join graphs and identify instances of cyclic join graphs that can be reduced to acyclic ones.

\footnotetext{
${ }^{1}$ See for instance (Selinger, Astrahan, Chamberlin, Lorie, and Price 1979), (Krishnamurthy, Boral, and Zaniolo 1986), (Swami and Iyer 1993), (Bayardo and Miranker 1996), and (Steinbrunn, Moerkotte, and Kemper 1997).
} 
This chapter is organized as follows. Section 9.1 gives a high level overview of our approach. In section 9.2 we give a generic cost model for computing a join, whose parameters are estimated in section 9.3. Next, in section 9.4, alternative methods for computing the optimal join order are investigated. Finally, section 9.5 discusses some remaining issues and future work.

\subsection{Motivation and approach}

To illustrate the join ordering problem, let us consider the following example:

Example 30 (join ordering). The program HOPCROFT (Listing 5.5 on page 88) implements the algorithm of Hopcroft (1971) for minimizing finite automata. It contains the following rule:

$\operatorname{part}(A, I), a(A, I, X), \operatorname{delta}(T, A, X) \backslash b(J, T) \Leftrightarrow b p(J, T)$.

In this rule, when part $(A, I)$ is activated, in order to achieve the correct time complexity, A and I should be used to find matching a (A, I,X) constraints; then $A$ and $X$ can be used to find matching $\operatorname{delta}(T, A, X)$ constraints; the resulting $T$ can finally be used to find a matching $\mathrm{b}(\mathrm{J}, \mathrm{T})$ constraint.

However, in many CHR systems, other join orderings are used for the rule in the above example, resulting in a worse time complexity. For instance, the current version of the Leuven CHR system uses A to find $\operatorname{delta}(T, A, X)$, then $T$ to find $\mathrm{b}(\mathrm{J}, \mathrm{T})$, and finally $\mathrm{A}, \mathrm{I}$ and $\mathrm{X}$ to find $\mathrm{a}(\mathrm{A}, \mathrm{I}, \mathrm{X})$. As a result, the optimal time complexity is not obtained. The heuristic introduced by Holzbaur et al. (2005) also results in a suboptimal join order - that is, if the functional dependencies ${ }^{2}$ $\operatorname{delta}(T, A, X)::\{T, A\} \rightsquigarrow\{X\}$ and $\mathrm{b}(J, T)::\{T\} \rightsquigarrow\{J\}$ are taken into account; if not, it will "coincidentally" pick the right join order.

Rules like that of Example 30, for which current CHR systems choose a suboptimal join order, motivate a closer investigation of the join ordering problem.

\subsubsection{Processing trees}

A join execution plan can be represented by a binary tree, called the processing tree. The leaves of a processing tree represent the different heads to be joined and its internal nodes represent the (partial) join of two or more heads. One particularly interesting type of processing trees are the so-called left-deep trees which are used by most database systems, as well as current CHR implementations. In particular, the standard compilation scheme of Section 4.2 uses left-deep processing trees.

\footnotetext{
${ }^{2}$ See Section 4.4 .4 on page 72 for more on functional dependencies.
} 


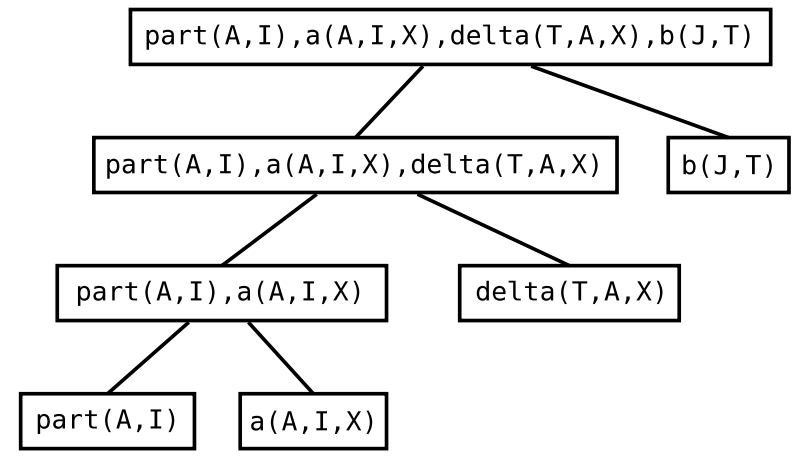

Figure 9.1: A left-deep processing tree for Example 30

Figure 9.1 shows the left-deep processing tree described above (in Example 30). Left-deep processing trees are inductively defined as follows: a single head is a leftdeep tree, and a tree in which the root node has a left-deep tree as its left child and a head as its right child is also a left-deep processing tree. A join proceeds by traversing all tuples of the left child of the join node and looking up appropriate tuples of the right child for each of them. This is often called a nested loop join.

Nested loop joins support pipelining: intermediate results do not need to be stored. In our investigation of join ordering we assume that partial joins are not indexed, i.e., if they are temporarily stored, we can only traverse them by sequential search. This implies that a right-deep processing tree (defined like a left-deep one, but with the left and right child switched) is problematic, since we have no indexing support to perform the nested loop joins.

The most general type of processing trees are called bushy trees. Both the left and right child of a bushy tree node can be a (non-trivial) processing tree itself. In general, bushy trees do not support pipelining and also using indexes is often a problem. However, there are cases in which these disadvantages are outweighed by reduced intermediate result sizes.

Example 31 (bad instance for left-deep processing trees). Consider a rule of the following form:

$$
a(X), b(X, Y), c(Y, Z), d(Z, A), e(A) \Leftrightarrow=>
$$

Assume the first head is the active constraint, and suppose there are many combinations of the second and third head resulting in the same value for $\mathrm{Z}$ and there are many $d / 2$ constraints for any value of its first argument, though few for which a matching e/1 constraint exists. Clearly, we can waste a lot of effort recomputing the join of $d(Z, A)$ and $e(A)$ for each combination of $b(X, Y)$ and $c(Y, Z)$. In this 


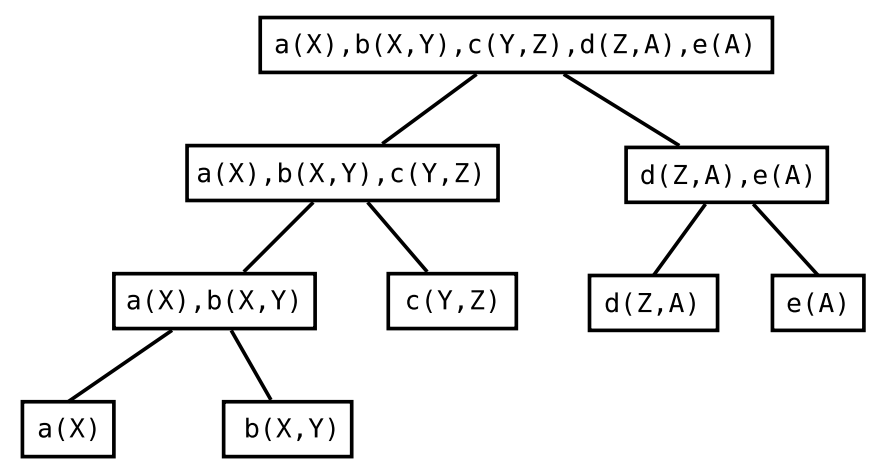

Figure 9.2: A bushy processing tree for Example 31

case it might be better to compute the join of $d(Z, A)$ and $e(A)$ once and store it for later retrieval. Figure 9.2 shows a bushy processing tree for this example.

From now on, we only consider left-deep processing trees.

(1) In cases like the above example, where a non-left-deep processing tree is needed, CHR programmers can manually simulate such processing trees by hard-coding partial joins. For instance, the rule of Example 31 can be changed as follows:

$d(Z, A), e(A) \Leftrightarrow \operatorname{de}(Z, A)$.

$a(X), b(X, Y), c(Y, Z), \operatorname{de}(Z, A) \Leftrightarrow \ldots$

\subsubsection{Static and dynamic join ordering}

Current CHR implementations attempt to determine an optimal join order (if they optimize at all) at compile time, after analyses such as functional dependency analysis (see Section 4.4.4). The join order therefore remains fixed during execution. We call this static join ordering.

Besides static join ordering, we also consider join ordering at run time: $d y$ namic join ordering. In the dynamic case, we can use runtime information such as constraint cardinalities and selectivities. However, at run time we cannot afford to spend as much time on optimization as at compile time.

Example 32 (need for dynamic join ordering). Consider the rule:

$\operatorname{supports}(A$, chelsea), $\operatorname{supports}(B$, liverpool) $\backslash$ friend $(A, B) \Leftrightarrow$ true.

Let there be $n$ supports/2 constraints, $\sqrt{n}$ of which have liverpool as their second argument. Let there be a functional dependency from the first argument of supports $/ 2$ to the second. 
Claim: In the above example, the optimal join ordering depends on the number of friend/ 2 constraints - which is only known at run time.

We consider two cases. In the first case, there are $n$ friend $/ 2$ constraints for every value of the first argument. In the second case, there are only a small constant number $(c)$ of friend/2 constraints for each such value. Each person initially has a representative number of friends supporting Liverpool, i.e., $\sqrt{n}$ in the first case and $\sqrt{c}$ in the second case.

Assume now that in the above example rule, the first head is active. There are only two candidate join orders: in $\Theta_{1}$, the second head is retrieved before the third, and in $\Theta_{2}$, the third head is retrieved before the second. Using the cost formula of Section 9.2, we find the following costs:

\begin{tabular}{c|c|c} 
Case & $C_{\Theta_{1}}$ & $C_{\Theta_{2}}$ \\
\hline 1 & $\sqrt{n}+\sqrt{n}$ & $n+\sqrt{n}$ \\
2 & $\sqrt{n}+\sqrt{c}$ & $c+\sqrt{c}$
\end{tabular}

Clearly, the wrong join order leads to a suboptimal complexity: $\Theta(n)$ versus $\Theta(\sqrt{n})$ in the first case and $\Theta(\sqrt{n})$ versus $\Theta(1)$ in the second. Moreover, the optimal join order depends on run-time statistics that could change during execution, so no static order can be optimal.

Dynamic join ordering allows a broad range of design choices. A join order should be available at the moment an active occurrence starts looking for partner constraints. However, after firing a first rule instance, run-time statistics may change and a different join order may become optimal. If we change the join order in between rule firings for the same active occurrence, we may no longer be able to use the partial joins that have already been computed, which probably outweighs the advantage of a better join order. Another issue is whether an optimal join order should be recomputed each time an occurrence becomes active. Clearly, a previously optimal join order remains optimal as long as run-time statistics do not change considerably, thus we can store it for later reuse.

\subsection{Cost model}

We now introduce a cost model to estimate the join cost of a particular join order. Our cost model assumes that rules are propagation rules with bodies that do not fundamentally change the relevant statistics, similar to what is assumed in the context of join order optimization for database systems. In the case of simplification or simpagation rules, it seems advantageous to optimize for finding the first full matches. Bayardo and Miranker (1996) handle this topic by taking into account the probability that a given partial match cannot be extended into a full match. We return to this issue in Section 9.5.1. 


\subsubsection{Notation}

Consider given an active identified constraint $a$, trying occurrence $H_{0}$ of a rule with $n$ remaining heads: $H_{1}, \ldots, H_{n}$. A join order $\Theta$ is a permutation of $\{0,1, \ldots, n\}$ such that $\Theta(0)=0$. The matching is done by nested partner lookups in the order $\Theta(1), \Theta(2), \ldots, \Theta(n)$. We use the notation st $\left(H_{i}\right)$ to denote all constraints (with identifier) in the CHR store which have the same constraint predicate as $H_{i}$. We use $\bar{h}$ to denote a tuple and $h_{i}$ to denote its $i$-th element (starting from 0 ). The rule guard $G$ determines which elements of st $\left(H_{1}\right) \times \ldots \times$ st $\left(H_{n}\right)$ are part of the join (we consider both the implicit and the explicit guard as part of $G$ ). Without loss of generality, this guard can be considered as a conjunction $g_{1}, \ldots, g_{m}$, where each conjunct $g_{j}$ is a host language constraint on a subset of the head variables. We define the guard scheduling index gs_index $\left(g_{j}\right)$ to be the earliest possible position in the join computation after which $g_{j}$ can be evaluated:

$$
\text { gs_index }\left(g_{j}\right)=\min \left\{k \in\{0, \ldots, n\} \mid \operatorname{vars}\left(g_{j}\right) \subseteq \bigcup_{i=0}^{k} \operatorname{vars}\left(H_{\Theta(i)}\right)\right\}
$$

If we have mode declarations for $g_{j}$, we can further refine this definition to consider only its input variables (Holzbaur, García de la Banda, Stuckey, and Duck 2005). We use $G^{k}$ to denote the part of $G$ that can be evaluated after the $k$-th lookup:

$$
G^{k}=\bigwedge\left\{g_{j} \mid 1 \leq j \leq m \wedge \text { gs_index }\left(g_{j}\right)=k\right\}
$$

We distinguish between two types of host language constraints in the guard: the first type are equality guards, the second type are other guards. The difference between both types of guards is that the first type can be evaluated in an a priori way: the equality can be used to do a (hash-table) lookup which means that partner constraints that do not satisfy the equality are not even considered - see Chapter 7 about indexing techniques. Constraints of the second type can only be evaluated in an a posteriori way: we consider all candidate partner constraints and check whether the guard holds. We denote the type- 1 guards of $G^{k}$ with $G_{\mathrm{eq}}^{k}$ and the type-2 guards with $G_{\star}^{k}$. We denote the built-in store with $\mathbb{B}$ and the built-in constraint theory with $\mathcal{D}$.

Our classification of guards as either a priori or a posteriori is implementationdependent. If more advanced indexing techniques are implemented, more kinds of guards are a priori guards. For example, in principle, if some Prolog predicate $\mathrm{p} / 1$ is used in a guard to test some property of a partner constraint argument, it might be useful to maintain a list of constraints that satisfy that test - especially if those are relatively sparse. If such a list is available, the guard $\mathrm{p} / 1$ is an a priori guard. See also the discussion in Section 7.5. 


\subsubsection{Partial joins}

Definition 9.1 (partial join) Given a built-in store $\mathbb{B}$, a CHR store described by $\operatorname{st}\left(H_{i}\right)$, a join order $\Theta$, and an active constraint $a$, we define $\mathcal{J}_{\Theta}^{k}$, the partial join up to the $k$-th partner, as follows: if $\mathcal{D} \not \models \mathbb{B} \rightarrow \bar{\exists}\left(G^{0} \wedge H_{0}=a\right)$, then $\mathcal{J}_{\Theta}^{k}=\emptyset$ for all $k$. Otherwise, $\mathcal{J}_{\Theta}^{k}$ is defined inductively: $\mathcal{J}_{\Theta}^{0}=\{a\}$ and $\mathcal{J}_{\Theta}^{k}=\mathcal{J}_{\Theta}^{k-1} \bowtie H_{\Theta(k)}$, where

$$
\mathcal{J}_{\Theta}^{k-1} \bowtie H_{\Theta(k)}=\left\{\bar{h} \in \mathcal{J}_{\Theta}^{k-1} \times \operatorname{st}\left(H_{\Theta(k)}\right) \mid \mathcal{D} \models \mathbb{B} \rightarrow \bar{\exists}\left(G^{k} \wedge \bigwedge_{i=0}^{k} h_{i}=H_{\Theta(i)}\right)\right\}
$$

The full join $\mathcal{J}_{\Theta}^{n}$ corresponds to the set of all tuples of matching partner constraints, and does not depend on the join order $\Theta$. We use $\mathcal{E}_{\Theta}^{k} \supseteq \mathcal{J}_{\Theta}^{k}$ to denote the partial join where in the last lookup, the condition is weakened to only the type-1 part of the guard:

$$
\mathcal{E}_{\Theta}^{k}=\left\{\bar{h} \in \mathcal{J}_{\Theta}^{k-1} \times \operatorname{st}\left(H_{\Theta(k)}\right) \mid \mathcal{D} \models \mathbb{B} \rightarrow \bar{\exists}\left(G_{\mathrm{eq}}^{k} \wedge \bigwedge_{i=0}^{k} h_{i}=H_{\Theta(i)}\right)\right\}
$$

\subsubsection{Cost formula}

The total cost for performing a join according to a join order $\Theta$ depends on the sizes of the intermediate partial joins $\mathcal{J}_{\Theta}^{k}$. The size $\left|\mathcal{J}_{\Theta}^{k}\right|$ of the partial join $\mathcal{J}_{\Theta}^{k}$ can be written as

$$
\left|\mathcal{J}_{\Theta}^{k}\right|=\left|\mathcal{J}_{\Theta}^{k-1}\right| \cdot \sigma_{\mathrm{eq}}(k) \cdot \mu(k) \cdot \sigma_{\star}(k)
$$

where

$$
\begin{gathered}
\sigma_{\mathrm{eq}}(k)=\left|\mathcal{A}_{\Theta}^{k}\right| /\left|\mathcal{J}_{\Theta}^{k-1}\right| \\
\mu(k)=\left|\mathcal{E}_{\Theta}^{k}\right| /\left|\mathcal{A}_{\Theta}^{k}\right| \\
\sigma_{\star}(k)=\left|\mathcal{J}_{\Theta}^{k}\right| /\left|\mathcal{E}_{\Theta}^{k}\right|
\end{gathered}
$$

and

$$
\mathcal{A}_{\Theta}^{k}=\left\{\bar{h} \in \mathcal{J}_{\Theta}^{k-1} \mid \exists h_{k} \in \operatorname{st}\left(H_{\Theta(k)}\right):\left(\bar{h}, h_{k}\right) \in \mathcal{E}_{\Theta}^{k}\right\}
$$

Intuitively, $\sigma_{\mathrm{eq}}(k)$ represents the percentage of tuples of the partial join $\mathcal{J}_{\Theta}^{k-1}$ for which the a priori lookup of the next partner constraint is successful, that is, at least one constraint exists in st $\left(H_{\Theta(k)}\right)$ that satisfies the equality guard. The intuitive meaning of $\mu(k)$ is the average multiplicity of a priori lookups of the next partner constraint, that is, the number of constraints from st $\left(H_{\Theta(k)}\right)$ that correspond to a given tuple from $\mathcal{J}_{\Theta}^{k-1}$ with respect to $G_{\text {eq }}^{k}$, averaged over all tuples $j \in \mathcal{J}_{\Theta}^{k-1}$ for which at least one such constraint exists. Finally, $\sigma_{\star}(k)$ intuitively corresponds to the percentage of tuples from $\mathcal{J}_{\Theta}^{k-1} \times H_{k}$ that satisfy the remaining guard $G_{\star}^{k}$ given that the equality guard $G_{\mathrm{eq}}^{k}$ is satisfied. 
Assuming that finding the set of partners that satisfy the a priori guard can be done in constant time, and assuming that evaluating the a posteriori guard also takes only constant time per tuple, the total cost of joining $n$ heads using the sequence of joins $\left(\left(\left(H_{\Theta(1)} \bowtie H_{\Theta(2)}\right) \bowtie H_{\Theta(3)}\right) \ldots \bowtie H_{\Theta(n)}\right)$ is proportional to $C_{\Theta}^{[1 . . n]}$, the sum of the a priori sizes of all partial joins:

$$
C_{\Theta}^{[1 . . n]}=\sum_{j=1}^{n} \frac{\left|\mathcal{J}_{\Theta}^{j}\right|}{\sigma_{\star}(j)}=\sum_{j=1}^{n} \prod_{k=1}^{j}\left(\sigma_{\star}(k-1) \cdot \sigma_{\mathrm{eq}}(k) \cdot \mu(k)\right)
$$

Clearly, different join orders have a different cost. It is our objective to find the join order with minimal cost.

\subsection{Approximating costs}

In this section we propose static and dynamic approximations of the cost model that was defined in the previous section.

\subsubsection{Static cost approximations}

Note that the following trivial inequalities always hold:

$$
\begin{gathered}
0 \leq \sigma_{\mathrm{eq}}(k) \leq 1 \\
0 \leq \sigma_{\star}(k) \leq 1 \\
1 \leq \mu(k) \leq\left|\operatorname{st}\left(H_{\Theta(k)}\right)\right|
\end{gathered}
$$

In the static approach we use the upper bound 1 as an estimate for $\sigma_{\text {eq }}(k)$. For $\sigma_{\star}(k)$ and $\mu(k)$ we will try to find a more accurate estimate.

\section{Estimating $\mu(k)$}

CHR constraints have multiset semantics in general, but in practice constraints have set semantics or at least multiplicities that are bounded by a small constant. If we can derive a functional dependency for $H_{\Theta(k)}$ given the variables of the previous heads $\bigcup_{i=0}^{k-1} \operatorname{vars}\left(H_{\Theta(i)}\right)$ and the equality guard $G_{\mathrm{eq}}^{k}$, then we know that there can be at most one $H_{\Theta(k)}$ constraint for a given tuple of $\mathcal{J}_{\Theta}^{k-1}$. So in that case, $\mu(k)=1$. Similarly, if we can derive a generalized functional dependency with multiplicity $m$, we get $1 \leq \mu(k) \leq m$ so we can estimate $\mu(k)$ to be in the middle of that interval: $\frac{m+1}{2}$.

In general, we propose the following heuristic to estimate $\mu(k)$ : Assume that $H_{\Theta(k)}$ is a constraint of arity $m$, and let $A=\left\{a_{1}, \ldots, a_{m}\right\}$ be its arguments. Let $K \subseteq A$ be the subset of arguments that are given by the previous heads and the 
equality guard $G_{\mathrm{eq}}^{k}$. Let $S \subseteq A$ be the subset of arguments that are declared or inferred to be ground and of a finite (and small) type. Let $f: 2^{A} \mapsto 2^{A}$ denote the reflexive transitive closure of the inferred functional dependency relation on the arguments of $H_{\Theta(k)}$. Now we define the set of candidate free argument sets $C=\{X \subseteq A \mid f(X \cup K \cup S)=A\}$ : these are subsets of arguments that, together with $K$ and $S$, determine all arguments. The set of reduced free argument sets $F \subseteq 2^{A}$ is defined inductively as the smallest set satisfying:

$$
\left\{\begin{array}{l}
X \in C \Longrightarrow X \in F_{K \backslash S} \\
X \in F_{Y} \wedge y \in Y \wedge x \in f^{-1}(y) \Longrightarrow(X \backslash\{x\}) \in F_{Y \backslash\{y\}} \\
F=\bigcup_{Y \subseteq(K \backslash S)} F_{Y}
\end{array}\right.
$$

Finally, we define $f$, the number of degrees of freedom of $H_{\Theta(k)}$, as the minimal size of a reduced free argument set: $f=\min _{X \in F}(|X|)$. As a heuristic, we estimate $\mu(k)$ to be $N^{f}$, where $N$ is some arbitrary constant, for instance $N=10$.

As an example, consider a constraint $\mathrm{c}$ with five arguments $c_{1}, \ldots, c_{5}$ with the type declaration $c$ (+int, ?int, ?int, +bool, -int). Because +bool is the only ground finite type, we have $S=\left\{c_{4}\right\}$. First assume there are no nontrivial functional dependencies for $\mathrm{c} / 5$. In an ordering like a, $\mathrm{c}\left({ }_{-},,_{-},,_{-},{ }_{-}\right)$, we have $K=\emptyset$, so we get $C=\left\{\left\{c_{1}, c_{2}, c_{3}, c_{5}\right\},\left\{c_{1}, c_{2}, c_{3}, c_{4}, c_{5}\right\}\right\}=F_{\emptyset}=F$ and $f=4$. Intuitively, there are 4 degrees of freedom to choose $c / 5$ : every argument with an infinite type contributes one degree of freedom. If $\mathrm{c} / 5$ has set semantics and the domain of its arguments is of size $N$ (at run-time), then there can be only $O\left(N^{4}\right)$ elements in st $(c / 5)$. In an ordering like $\mathrm{a}(\mathrm{A}), \mathrm{b}(\mathrm{B}, \mathrm{C}), \mathrm{c}\left(\mathrm{A}, \mathrm{B},,_{-},{ }_{-}\right)$, we have $K=\left\{c_{1}, c_{2}\right\}$, so now we get $C=\left\{\left\{c_{3}, c_{5}\right\}, \ldots\right\}=F_{K}=F$ and there are only two degrees of freedom: $f=2$. Intuitively, given the first two arguments, there can be only $O\left(N^{2}\right)$ matches.

Now assume $\mathrm{c} / 5$ has a functional dependency from its first two arguments to its third argument - e.g. because the program contains the following rule:

$$
c\left(A, B, X,_{-},-\right), c\left(A, B, Y,,_{-}\right)==X=Y \text {. }
$$

Consider the same ordering $a(A), b(B, C), c\left(A, B,,_{-},,_{-}\right)$. Because of the functional dependency we now have $\left\{c_{5}\right\} \in C=F$ and $f=1$ : given the first two arguments, the third argument is also fixed, so there are only $O(N)$ matches. Finally, consider the ordering $\mathrm{b}(\mathrm{B}, \mathrm{C}), \mathrm{c}\left({ }_{-},{ }_{-}, \mathrm{C},,_{-},{ }_{-}\right)$. We have $K=\left\{c_{3}\right\}$ and $\left\{c_{1}, c_{2}, c_{5}\right\} \in C=F_{\left\{c_{3}\right\}}$. Now because $c_{1} \in\left\{c_{1}, c_{2}\right\} \in f^{-1}\left(c_{3}\right)$, we have $\left\{c_{2}, c_{5}\right\} \in$ $F_{\emptyset}$. (Similarly, because $c_{2} \in\left\{c_{1}, c_{2}\right\} \in f^{-1}\left(c_{3}\right), F_{\emptyset}$ contains $\left\{c_{1}, c_{5}\right\}$.) Hence there are only two degrees of freedom: $f=2$.

This may seem somewhat counterintuitive at first. The reasoning behind it is as follows: there are $O\left(N^{2}\right)$ combinations of the first two arguments, and every combination maps to one of the $O(N)$ values of the third argument, so on average, when we fix a value of the third argument, there are only $O\left(\frac{N^{2}}{N}\right)=O(N)$ possibilities for the combination of the first two arguments. So instead of letting both 
$c_{1}$ and $c_{2}$ contribute one degree of freedom, we count only one of them. Of course this argument is only valid under some rather strong assumptions; for instance, it fails miserably if most combinations of $c_{1}$ and $c_{2}$ result in the same value of $c_{3}$ and if precisely that value is tried. We expect to get a reasonable heuristic when the argument domains are sufficiently similar in size and the distribution of tried values for the target of the functional dependency can be approximated to be uniform.

\section{Estimating $\sigma_{\star}(k)$}

If the a posteriori guard $G_{\star}^{k}$ is entailed by $G^{k-1}$ (the guards with lower guard scheduling index), then $\sigma_{\star}(k)=1$. In particular, this is the case for rules without (explicit) guards. Similarly, if the negation of $G_{\star}^{k}$ is entailed by $G^{k-1}$, then $\sigma_{\star}(k)=$ 0 . If this is the case, then the rule most likely represents a bug in the CHR program since it cannot fire (cf. Chapter 6).

In the other cases, we can use the following heuristic. Let $G_{\star}^{k}=g_{1}, \ldots, g_{m}$. As an estimate for $\sigma_{\star}(k)$, we use the following product: $\sigma_{\star}\left(g_{1}\right) \cdot \ldots \cdot \sigma_{\star}\left(g_{m}\right)$, where $\sigma_{\star}\left(g_{i}\right)=0.5$ if $g_{i}$ is of the form _ $<_{-},>_{-},>_{-}=<_{-}$or $>_{-}=_{-} ; \sigma_{\star}\left(g_{i}\right)=0.25$ if $g_{i}$ is of the form _ $=:=_{-}$or $\_$is _ $; \sigma_{\star}\left(g_{i}\right)=0.95$ if $g_{i}$ is of the form _ $=\backslash=_{-}$or $\_~ \backslash==_{-}$; and $\sigma_{\star}\left(g_{i}\right)=0.75$ otherwise. Of course this heuristic is very arbitrary and ad-hoc, and it can be extended or modified. The idea is that disequalities usually hold, (arithmetic) equalities usually do not, and less-than(-or-equal) usually holds in half of the cases. Unknown guards are assumed to hold in $75 \%$ of the cases, and guards in conjunction are treated as if they independently filter the valid partner constraints.

\subsubsection{Dynamic cost approximations}

In the dynamic approach, we maintain statistics about the constraint store and use them to find upper bounds and approximations of the cost of join orders.

\section{Worst-case bounds}

In the worst case, the trivial upper bound of 1 is reached for both $\sigma_{\mathrm{eq}}(k)$ and $\sigma_{\star}(k)$. For $\mu(k)$ we can obtain a tighter upper bound by maintaining, for every data structure which is used for constraint lookup, the maximal number of constraints per lookup key - e.g., for hash-tables this corresponds to the maximal bucket size. The equality guard $G_{\mathrm{eq}}^{k}$ determines the data structure that will be used for the lookup. The maximal number of constraints per key of that data structure is hence an upper bound for $\mu(k)$. 


\section{Approximations}

Instead of maintaining the maximal number of constraints per key, we can also easily maintain the average number of constraints per key. This average is a good approximation of $\mu(k)$ if we assume that the effectively used lookup keys are representative. As an approximation of $\sigma_{\star}(k)$ we can either use the same heuristic as in the static case, or estimate the value by dynamically maintaining the success rate of the type- 2 guard. Finally, we approximate $\sigma_{\mathrm{eq}}(k)$ as follows. We maintain the total number of distinct keys in every constraint data structure, and we compute the size of the key domain, as follows: for every type used in constraint arguments, we maintain the type domain size as the number of distinct (ground or nonground) values that were effectively encountered in arguments of that type. The size of a single-argument key domain is simply the domain size of its type. For multi-argument keys we use the product of the corresponding type domain sizes. In a sense, $\sigma_{\mathrm{eq}}(k)$ represents the chance that a key exists (i.e., lookup on a key is non-empty). We approximate it by dividing the number of keys by the size of the key domain. This is reasonable assuming that the keys tried are randomly sampled from the key domain.

\section{Hybrid heuristic}

In practice, it makes sense to use a weighted sum of the above approximation and the worst-case bound. If the approximation results in a $\operatorname{cost} C_{a}$ and the worst-case bound results in a cost $C_{w}$, we may want to optimize $(1-\alpha) \cdot C_{a}+\alpha \cdot C_{w}$, where $\alpha$ is some small constant, e.g., $\alpha=0.05$.

\subsection{Optimization}

This section discusses algorithms that can be used to determine the optimal join order given our cost model and approximations of its parameters. Section 9.4.1 introduces the notion of join graphs. In section 9.4.2, an $O(n \log n)$ algorithm for the special case of acyclic join graphs is presented (where $n$ is the number of heads). Section 9.4.3 deals with the general case of cyclic join graphs. The results given are applicable for both static and dynamic join ordering. In the static case, we generally have less stringent time constraints, but the parameter estimations of the cost model are also generally less accurate. On the other hand, in the dynamic case, our model can be expected to be more accurate, but computing the optimal join order using exact algorithms may be prohibitively expensive. Therefore, in section 9.4.4, we compare the cost of joining in the optimal join order with the cost of finding this optimal join order. 


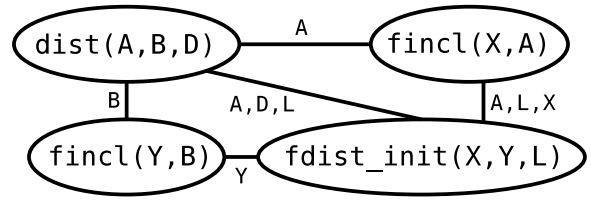

(a) Cyclic join graph

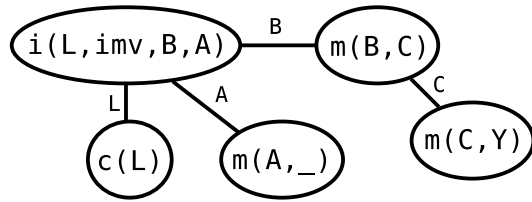

(b) Acyclic join graph

Figure 9.3: Cyclic and acyclic join graphs

\subsubsection{Join graphs}

The join graph for a given rule is constructed as follows: the vertices correspond to the heads of the rule and the edges link heads whose variables are connected via guards - including, of course, the implicit equality guards. Join graphs are cyclic or acyclic.

Example 33 (cyclic and acyclic join graphs). Figure 9.3 shows an example of both a cyclic and an acyclic join graph. The cyclic join graph in figure 9.3(a) corresponds to the following rule (from the timed_automata program):

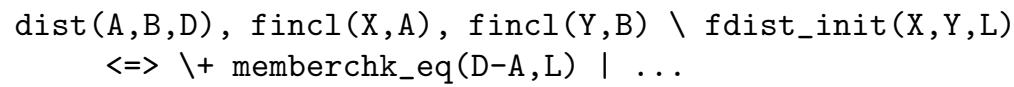

Figure 9.3(b) shows the acyclic join graph corresponding to the following rule (from the RAMSIMUL program on page 221):

$$
i(L, i m v, B, A), m(B, C), m(C, Y) \backslash m\left(A,{ }_{-}\right), c(L) \Leftrightarrow \ldots
$$

\subsubsection{Acyclic join graphs}

In this section, we show how the optimal join ordering can be found in $O(n \log n)$ time for $n$-headed rules with an acyclic join graph. This result is based on results from database query optimization. An acyclic join graph can be represented as a tree with the active constraint as its root node. The algorithm proposed in this section returns an optimal join order if the following assumptions hold:

1. The optimal join order respects the tree order, i.e., no head is looked up before its parent in the join tree.

2. All selections are representative: given a join and a head $H$ extending the join, then adding or removing other heads to/from the join does not influence the selectivity and multiplicity for $H$. 
Given a join order $\Theta$ and assume that for given $j$, join order $\Theta^{\prime}$ is cheaper, where $\Theta^{\prime}$ equals $\Theta$ except that the $j$-th and $(j+1)$-th elements are switched, i.e., $\Theta^{\prime}=$ $\Theta \circ(j, j+1)$. Since under our assumptions, $\sigma_{\star}(i), \sigma_{\mathrm{eq}}(i)$ and $\mu(i)$ only depend on the parent of the $i$-th head in the join tree, we have that

$$
\begin{aligned}
C_{\Theta^{\prime}}-C_{\Theta} & =C_{\Theta}^{[1 \ldots j-1]} \cdot\left(\sigma_{\star}(j-1) \cdot \rho(j+1) \cdot\left(1+\sigma_{\star}(j+1) \cdot \rho(j)\right)\right. \\
& \left.-\sigma_{\star}(j-1) \cdot \rho(j) \cdot\left(1+\sigma_{\star}(j) \cdot \rho(j+1)\right)\right) \leq 0
\end{aligned}
$$

where $\rho(i)=\sigma_{\mathrm{eq}}(i) \cdot \mu(i)$ and $C_{\Theta}=C_{\Theta}^{[1 . . n]}$. We further simplify this as follows:

$$
\begin{gathered}
\rho(j+1)+\rho(j) \cdot \rho(j+1) \cdot \sigma_{\star}(j+1) \leq \rho(j)+\rho(j+1) \cdot \rho(j) \cdot \sigma_{\star}(j) \\
\rho(j+1) \cdot\left(1-\rho(j) \cdot \sigma_{\star}(j)\right) \leq \rho(j) \cdot\left(1-\rho(j+1) \cdot \sigma_{\star}(j+1)\right)
\end{gathered}
$$

Because $\rho(i) \geq 0$ for any $i$ :

$$
\frac{\rho(j+1) \cdot \sigma_{\star}(j+1)-1}{\rho(j+1)} \leq \frac{\rho(j) \cdot \sigma_{\star}(j)-1}{\rho(j)}
$$

We expand the comparison measure towards sequences: sequences $s$ and $t$ can be switched if and only if

$$
C_{s}+\left|\mathcal{J}_{s}\right| \cdot C_{t} \leq C_{t}+\left|\mathcal{J}_{t}\right| \cdot C_{s}
$$

where $\mathcal{J}_{\Theta}=\mathcal{J}_{\Theta}^{n}$. This condition can also be expressed in terms of ranks of sequences, as in the approach of Ibaraki and Kameda (1984) and Krishnamurthy et al. (1986):

$$
\operatorname{rank}(s)=\frac{\left|\mathcal{J}_{s}\right|-1}{C_{s}} \leq \frac{\left|\mathcal{J}_{t}\right|-1}{C_{t}}=\operatorname{rank}(t)
$$

Now, consider a head $H_{i}$ whose direct parent $H$ has a higher rank. We can show that only descendants of this parent are allowed between $H$ and $H_{i}$. Assume that another head $H_{j}$ appears in between $H$ and $H_{i}$ in the optimal order. Since $H_{j}$ is not a descendant of $H$, it can be placed before $H$. Assume it has a lower rank than $H$, then we can move it from in between $H$ and $H_{i}$ to before $H$ and the resulting cost is smaller. If it has a higher rank than $H$, then it also has a higher rank than $H_{i}$ and it can be moved to after $H_{i}$, resulting again in a smaller cost. In conclusion, if a head has lower rank than its parent, then only descendants of this parent can be in between the head and its parent in the optimal join order.

Figure 9.4 shows the algorithm of (Krishnamurthy, Boral, and Zaniolo 1986), which outputs a single chain representing the optimal join order. To illustrate the algorithm, consider the following example:

Example 34 (KBZ algorithm). Consider the following rule:

$$
\text { h1 (A,B), h2 (A,C), h3(B,D), h4 (D), h5(C) ==> . . }
$$

Assuming the first head is active, we get the (directed) join graph of Figure 9.5, in which an edge to $\mathrm{h} i$ is annotated with $\sigma_{\mathrm{eq}}(\mathrm{h} i) \cdot \mu(\mathrm{h} i)$. 
Step 1. If the tree is a single chain then stop.

Step 2. Find a subtree all of whose children are chains. Let $r$ be its root.

Step 3. Merge the chains based on ranks such that the resulting single chain is nondecreasing on the rank.

Step 4. Normalize the root $r$ of the subtree as follows: while the rank of the root $r$ is greater than the rank of its immediate child $c$ : replace $r$ and $c$ by a new node representing the subchain $r$ followed by $c$.

Step 5. Go to Step 1.

Figure 9.4: KBZ join ordering algorithm for acyclic join graphs

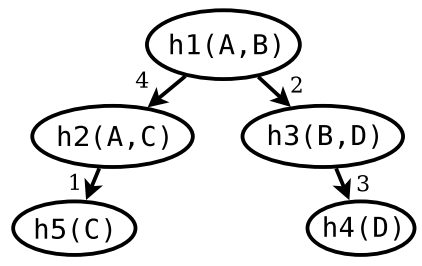

\begin{tabular}{c|c|c|c} 
Head & $|\mathcal{J}|$ & $C$ & Rank \\
\hline h2 & 4 & 4 & $3 / 4$ \\
h3 & 2 & 2 & $1 / 2$ \\
h4 & 3 & 3 & $2 / 3$ \\
h5 & 1 & 1 & 0
\end{tabular}

Figure 9.5: Join graph and ranks for Example 34

Assume, for the above example, that we start with the textual order as initial join order:

$$
C_{[1,2,3,4,5]}=4+4 \cdot 2+4 \cdot 2 \cdot 3+4 \cdot 2 \cdot 3 \cdot 1=60
$$

Since $\operatorname{rank}(\mathrm{h} 5)<\operatorname{rank}(\mathrm{h} 2)$, but h2 should be before h5 according to the tree order, we have that no heads should be scheduled in between h2 and h5. Indeed, since $\operatorname{rank}(\mathrm{h} 3)<\operatorname{rank}(\mathrm{h} 2)$ it is cheaper to put h3 before h2:

$$
C_{[1,3,2,4,5]}=2+2 \cdot 4+2 \cdot 4 \cdot 3+2 \cdot 4 \cdot 3 \cdot 1=58
$$

and similarly for h4 and h2:

$$
C_{[1,3,4,2,5]}=2+2 \cdot 3+2 \cdot 3 \cdot 4+2 \cdot 3 \cdot 4 \cdot 1=56
$$

Now h2 is directly before h5 and we combine both into a new node with $\operatorname{rank}([\mathrm{h} 2, \mathrm{~h} 5])=$ $3 / 8$. For the new node, we have that $\operatorname{rank}([\mathrm{h} 2, \mathrm{~h} 5])<\operatorname{rank}(\mathrm{h} 4)$ and so we can switch them:

$$
C_{[1,3,2,5,4]}=2+2 \cdot 4+2 \cdot 4 \cdot 1+2 \cdot 4 \cdot 1 \cdot 3=42
$$

and similarly for $[\mathrm{h} 2, \mathrm{~h} 5]$ and h3:

$$
C_{[1,2,5,3,4]}=4+4 \cdot 1+4 \cdot 1 \cdot 2+4 \cdot 1 \cdot 2 \cdot 3=40
$$

Now all heads (sequences) are sorted by rank and the join order is optimal. 


\subsubsection{Cyclic join graphs}

While we have an efficient algorithm for finding the optimal join order for acyclic join graphs, the same optimization problem for general graphs has been proven to be NP-complete by Ibaraki and Kameda (1984). The standard approach consists of applying an exponential algorithm based on dynamic programming. Alternatives include applying the algorithm for acyclic join graphs to various spanning trees of the join graph, as done by Krishnamurthy et al. (1986) and Swami and Iyer (1993), and using randomized algorithms like simulated annealing and genetic algorithms (Steinbrunn, Moerkotte, and Kemper 1997).

\section{Exact algorithm}

The exact algorithm considers all permutations of the heads and makes use of the fact that the order of an initial subsequence of the heads in optimal join order is also an optimal join order for this subsequence:

$$
C_{s++t}=C_{s}+\left|J_{s}\right| \cdot \sum_{j=|s|}^{n-1} \prod_{k=|s|}^{j}\left(\sigma_{\star}(k) \cdot \sigma_{\mathrm{eq}}(k+1) \cdot \mu(k+1)\right)
$$

The second part of the sum is independent of the order of $s$.

The task at hand is to find the shortest path in a weighted directed acyclic graph (DAG) of the following form:

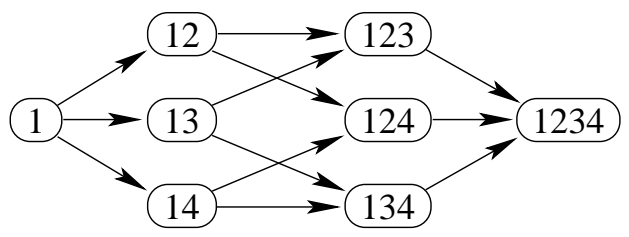

The graph has $2^{n}$ nodes and $n \cdot 2^{n-1}$ edges. We can now use a single source shortest path algorithm for DAGs (based on topological order) which has a runtime proportional to the number of edges in the graph, i.e., $\mathcal{O}\left(n \cdot 2^{n}\right)$. An often used heuristic is to avoid Cartesian products by postponing choosing heads which have no variables in common with the partial join computed so far, i.e., only those nodes connected to the partial join in the join graph are considered unless this is not possible (Selinger, Astrahan, Chamberlin, Lorie, and Price 1979). This can however lead to suboptimal solutions. 
Example 35 (avoiding Cartesian product can be bad). To illustrate how the heuristic of avoiding Cartesian products can lead to a suboptimal solution, consider the following rule:

$a(X), b(X, Y), c(Y)==>\ldots$

Assume a constraint store containing many $\mathrm{b} / 2$ constraints for any given value of its first argument, and few $c / 1$ constraints. Let the first head be the active constraint. The second head has a variable in common with the active constraint whereas the third head has not. However, it is more interesting to lookup the third head first, as this drastically limits the amount of $\mathrm{b} / 2$ constraints considered for the second head.

As an improvement on the simple shortest path algorithm, we can use the $\mathrm{A}^{*}$ algorithm (Hart, Nilsson, and Raphael 1968) by making an estimate of the cost of joining the remaining heads, given a partial join. Clearly, we want the heuristic to be computationally cheap. A very simple estimate for the remaining cost is the size of the total join, but this size is the same for all join orders, so it does not contribute to the search. If we make the assumption of representative selection, as we did for the case of acyclic join graphs, as well as in our estimates of actual selectivity and multiplicity, then we can use the following formula:

$$
C_{s++t} \geq C_{s}+\left|J_{s}\right| \cdot \sum_{j=|s|}^{n-1} \prod_{k=|s|}^{j}\left(\sigma_{\star}^{\prime}(k) \cdot \sigma_{\mathrm{eq}}^{\prime}(k+1) \cdot \mu^{\prime}(k+1)\right)
$$

where $\sigma_{\star}^{\prime}, \sigma_{\text {eq }}^{\prime}$ and $\mu^{\prime}$ are defined similarly as $\sigma_{\star}, \sigma_{\text {eq }}$ and $\mu$, but also take into account the information from the remaining heads. The join order that minimizes this underestimate (and hence is an underestimate for any order of the remaining heads $t$ ) is the one in which the heads of $t$ are ordered by increasing $\sigma_{\text {eq }}^{\prime} \cdot \mu^{\prime} \cdot \sigma_{\star}^{\prime}$. Moreover, we have that the optimal order for $t$ projected on a subset of $t$, is also an optimal order for this subset. This implies that we can compute the optimal order for the full join once and only need to select the relevant terms during search. The complexity of calculating the heuristic lower bound is hence proportional to the number of heads $n$.

Since the exact algorithm has an exponential time and space complexity, and the $\mathrm{A}^{*}$ algorithm is not better in the worst case, we investigate some approximative alternatives in the next subsections.

\section{Minimal spanning tree}

In the approach of Krishnamurthy et al. (1986), cyclic join graphs are first made acyclic by constructing a minimal spanning tree and applying the algorithm for acyclic graphs on the resulting join tree. The edge weights used are the selectivities. Swami and Iyer (1993) propose a more complex solution. It consists of applying the 
algorithm for acyclic join graphs to a (random) spanning tree, followed by a phase of iterative improvement in which pairs of heads are switched. This process is repeated multiple times with randomized starting points. In any case, the optimal solution found using any spanning tree is not necessarily optimal for the complete join graph.

\section{Randomized algorithms}

Given that we use approximations for $\sigma_{\star}, \sigma_{\text {eq }}$ and $\mu$, the optimal join order found using exact algorithms is in fact still an approximation. This motivates the use of randomized algorithms that give approximatively optimal solutions to the inexact optimization problem. An overview of such randomized algorithms can be found in (Steinbrunn, Moerkotte, and Kemper 1997). They include iterative improvement or simulated annealing from random initial join orders, and genetic algorithms.

\subsubsection{Optimization cost versus join cost}

While some solutions for the optimization problem, in particular the exact algorithm for cyclic join graphs, may appear to be prohibitively expensive, it should always be compared to the cost of actually performing the join. Assume for example that in the optimal join order, $\sigma_{\star}(k-1) \cdot \sigma_{\mathrm{eq}}(k) \cdot \mu(k) \geq 2$ for all $k \in\{1, \ldots, n\}$, then the cost of performing the join is $\Omega\left(2^{n}\right)$ and so the optimization cost may not be such a problem. Moreover, the time spent on optimization should always be compared with the join cost when no optimization took place. One solution to this optimization cost dilemma is to first optimize using a fast but suboptimal algorithm (e.g., a greedy algorithm). We should only optimize using an exact algorithm if the expected optimization cost is less than the join cost of the plan resulting from the first optimization step.

\subsection{Discussion}

\subsubsection{First-few answers}

As already pointed out in section 9.2 , our cost model is based on the assumption that all fireable rule instances eventually fire. In many cases this assumption obviously does not hold. Consider for example a simplification or simpagation rule in which the active constraint is removed. Clearly, only the first rule matching which is found will actually fire. Therefore it can be advantageous to optimize for finding the first answer. We now construct an alternative cost model, which represents the cost for finding the first full match. This model is based on the work of Bayardo and Miranker (1996).

Let $p(i)$ denote the probability that a tuple from the partial join up to the $i$-th head (in some order $\Theta$ ) can be extended to a full join tuple. We recursively define 


\begin{tabular}{|l|c|c|cc|cc|cc|cc|cc|}
\hline Program & $n=1$ & $n=2$ & \multicolumn{2}{|c|}{$n=3$} & \multicolumn{2}{|c|}{$n=4$} & $n=5$ & $n=6$ & \multicolumn{2}{|c|}{ Total } \\
\hline car_rental & 5 & 4 & 2 & $(0)$ & 5 & $(4)$ & 2 & $(2)$ & & & 18 & $(6)$ \\
HOPCROFT & 6 & 7 & 2 & $(0)$ & 2 & $(2)$ & & & & 17 & $(2)$ \\
monkey_banana & 1 & 7 & 15 & $(7)$ & 2 & $(0)$ & & & & & 25 & $(7)$ \\
RAMSIMUL & 1 & 2 & 3 & $(0)$ & 5 & $(0)$ & 2 & $(0)$ & & & 13 & $(0)$ \\
timed_automata & & 11 & 10 & $(9)$ & 3 & $(3)$ & & & & & 24 & $(12)$ \\
inference & 26 & 48 & 13 & $(7)$ & 6 & $(5)$ & 4 & $(4)$ & & & 97 & $(16)$ \\
wellfounded_sem & 3 & 25 & 8 & $(1)$ & 4 & $(2)$ & 1 & $(1)$ & 2 & $(2)$ & 43 & $(6)$ \\
\hline Total & 42 & 104 & 53 & $(24)$ & 27 & $(16)$ & 9 & $(7)$ & 2 & $(2)$ & 237 & $(49)$ \\
\hline
\end{tabular}

Table 9.1: Number of rules with $n$ heads (with cyclic join graphs)

$p(i)$ as follows:

$$
p(i)=\sigma_{\mathrm{eq}}(i) \cdot\left(1-(1-p(i+1))^{\mu(i) \cdot \sigma_{\star}(i)}\right)
$$

It is the probability that the tuple can be extended by at least one head $\left(\sigma_{\text {eq }}(i)\right)$, times the probability that at least one of the tuples of $i+1$ heads survives (i.e., one minus the probability that all fail). The total cost of finding the first answer can then be written as follows:

$$
C=\sum_{i=1}^{n} \frac{1}{p(i)}
$$

Here we assume that $1 / p(i)$ never exceeds $\left|J_{i}\right|$. The reasoning is as follows: $p(i) \cdot\left|J_{i}\right|$ tuples out of $\left|J_{i}\right|$ will participate in a full match. Under the assumption that these tuples are uniformly distributed amongst all tuples, we will need to skip $1 / p(i)$ tuples before finding the first tuple that takes part in a full match.

For more general types of rules, we can make a weighted sum of the cost model introduced here, and the one from section 9.2 .

\subsubsection{Cyclic and acyclic join graphs}

Table 9.1 shows for some example programs the number of $n$-headed rules and between brackets the number of rules with cyclic join graphs.

Not every edge in a join graph has the same importance, as we will illustrate with a few examples.

Example 36 (weak edge). Consider the rule (from inference)

$$
\operatorname{st}(\mathrm{S}, \mathrm{T} 1), \operatorname{st}(\mathrm{S}, \mathrm{T} 2) \backslash \operatorname{rt}(\mathrm{T} 1, \mathrm{R} 1), \operatorname{rt}(\mathrm{T} 2, \mathrm{R} 2) \Leftrightarrow \mathrm{R} 1 \backslash==\mathrm{R} 2 \mid \ldots
$$

The disequality guard creates a cycle in the join graph. However, its influence on the optimal join order is expected to be minimal, i.e., we can assume that it is better to lookup $\mathrm{rt}(\mathrm{T} 2, \mathrm{R} 2)$ via $\mathrm{T} 2$ than using $\mathrm{R} 1 \backslash==\mathrm{R} 2$ given $\mathrm{R} 1$. 

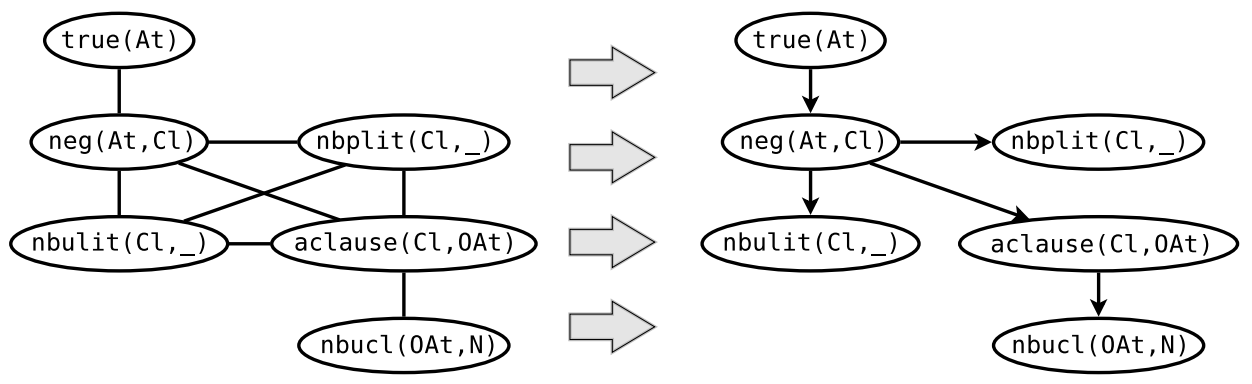

Figure 9.6: Eliminating a clique in a join graph

We can expect that "weak" edges (e.g. edges induced by a disequality guard) can be safely removed from the join graph. This could break a cycle.

Example 37 (clique). Consider the rule (from wellfounded_sem)

true (At) \neg(At,Cl), aclause $(\mathrm{Cl}, \mathrm{OAt})$, $\operatorname{nbulit}\left(\mathrm{Cl}_{-}\right), \operatorname{nbplit}\left(\mathrm{Cl}_{-}\right), \operatorname{nbucl}(\mathrm{OAt}, \mathrm{N}) \Leftrightarrow \ldots$

The join graph is cyclic because variable $\mathrm{Cl}$ appears in more than two heads. However, once we have $\mathrm{Cl}$, looking up another head containing it does not give us any new information.

Under the assumption of representative selection that we made for the approximations of the cost model parameters, we can turn the join graph of the above example into a rooted join tree — one for every possible active constraint. Figure 9.6 depicts the original join graph (heads are numbered in textual order) and its transformation into a rooted join tree (where the first head constraint is active).

The idea is as follows: if the join graph contains a clique such that every two nodes in the clique share exactly the same variables, and moreover it holds that from any node outside of the clique, there is at most one path to any node of the clique, then given a root node, there exists a unique entry point into the clique (possibly the root node belongs to the clique itself) and we can remove all edges in the clique except those connected to this entry point. However, if some nodes of a clique share more variables than others, we cannot make the join graph acyclic.

Example 38 (bad clique). Consider the rule (from monkey_banana)

goal $(\mathrm{a}, \mathrm{h}, \mathrm{A}, \mathrm{B}, \mathrm{C})$, phys_object $(\mathrm{A}, \mathrm{E}, \mathrm{I}, \mathrm{n}, \mathrm{F}, \mathrm{G}, \mathrm{H})$, monkey $(\mathrm{E}, \mathrm{J}, \mathrm{A})==>\ldots$

Variable A appears in all three heads, but the second and third head also share variable E. Hence we cannot reduce the join graph to a rooted join tree. 
For the example programs of Table 9.1, only 10 of the 49 cyclic graphs remain cyclic after excluding the above two types of cyclicity.

\subsubsection{Join ordering in current CHR implementations}

Current CHR implementations generally follow the HAL heuristic of Holzbaur et al. (2005) or a variant of it, if they apply join ordering at all - some systems, for example Haskell CHR or the ECL ${ }^{i} \mathrm{PS}^{e}$ ech library (Wallace, Novello, and Schimpf 1997) simply use the textual order of heads. The Leuven CHR system uses a heuristic that was originally based on the HAL heuristic, but has become more complex over time due to incremental compiler changes.

The cost model for join ordering in the HAL CHR system is based on the assumption that all constraint arguments range over a domain of the same size $s$. Given a partial join $\mathcal{J}_{\Theta}^{i}$, let $w_{i}$ be the number of arguments of $H_{\Theta(i+1)}$ that are not fixed by $\mathcal{J}_{\Theta}^{i}$ (degree of freedom). The worst case size of $\mathcal{J}_{\Theta}^{i+1}$ is $\left|\mathcal{J}_{\Theta}^{i}\right| \cdot s^{w_{i}}=$ $s^{w_{1}+\ldots+w_{i}}$ In order to eliminate the domain size $s$, the cost of the total join is approximated by $\sum_{k=1}^{n-1}(n-k) w_{k}$ which is in fact the logarithm (to base $s$ ) of $\prod_{k=1}^{n}\left|\mathcal{J}_{\Theta}^{k}\right|$ whereas the real cost would be the sum of these partial join sizes. The weights relate to our approach as follows: $\mu(i) \approx s^{w_{i}}$. The degree of freedom is reduced by functional dependencies and explicit equality guards. For other guards, the reduction depends on the determinism of the guard predicate. For example, if the predicate is declared as semidet, then the degree of freedom is reduced by 0.25 per fixed argument. The motivation is a guard $\mathrm{X}>\mathrm{Y}$ which would remove 0.5 degrees of freedom. Note though that this implies a cost $s^{1.5}$ for a constraint $\mathrm{c}(\mathrm{X}, \mathrm{Y})$ with guard $\mathrm{X}>\mathrm{Y}$ whereas a more accurate estimate would be $s^{2} / 2$.

Finally, we note that although it is mentioned by Duck (2005) and Holzbaur et al. (2005) that often only the first full match is needed (cf. Section 9.5.1), the HAL cost model does not reflect this concern.

\subsubsection{Future work}

In this chapter, we have only looked at the theoretical side of the join ordering problem. Although it is already clear that a good join order, and even dynamic join ordering can result in a better runtime complexity, it remains an open question whether it is worth the extra effort in typical problems. Therefore, we plan to implement the proposed join ordering schemes. This will also allow us to experiment with ideas such as computing optimal join orders in parallel using a different thread, and hybrid static and dynamic join ordering (where the dynamic search space is reduced at compile time, e.g., based on the join graph or functional dependency analysis). Other issues we plan to investigate are the trade-off between optimization cost and join cost, using profiling information for static join ordering, and other criteria (apart from those given in section 9.5.2) to reduce a cyclic join graph to an acyclic one. 


\section{Part III}

\section{Computability and Complexity of CHR}





\title{
Chapter 10
}

\section{CHR Machines}

\author{
"A mathematician is a device for turning coffee into theorems." \\ — Paul Erdös (1913-1996)
}

\begin{abstract}
"Computer Science is no more about computers than astronomy is about telescopes."

"The question of whether Machines Can Think ... is about as relevant as the question of whether Submarines Can Swim."
\end{abstract}

— Edsger Dijkstra (1930-2002)

In the final part of this dissertation we study the complexity of CHR. We will derive a general complexity meta-theorem in Chapter 11 which we use to show a complexity-wise completeness result: we demonstrate that it is possible to implement any algorithm in CHR in an efficient way, i.e. with the best known time and space complexity. In Chapter 11.4 we investigate to what extent the result can be 'ported' to other declarative languages. Chapter 11.5 examines constant factors and Chapter 12 deals with some more exotic variants of CHR like nondeterministic and self-modifying CHR programs.

Our approach is as follows. We introduce a new model of computation, the CHR machine, and compare it with the standard models of computation - Turing machines and RAM machines (cf. Chapter 2). We define CHR machines in Section 10.1. Next, in Section 10.2 we show the unsurprising result that CHR machines are Turing-complete. In Section 10.3 we identify some syntactical subsets of CHR programs that are still Turing-complete, and some that are not. Finally, in Section 10.4 we define the time and space complexity of CHR machines. 


\subsection{Definition of CHR machines}

We define a CHR machine as follows:

Definition 10.1 (CHR machine) A CHR machine is a tuple $\mathcal{M}=(\mathcal{H}, \mathcal{P}, \mathcal{V G})$. The host language $\mathcal{H}$ defines a built-in constraint theory $\mathcal{D}_{\mathcal{H}}, \mathcal{P}$ is a $C H R$ program, and $\mathcal{V G} \subseteq \mathcal{G}_{\mathcal{P}}^{\mathcal{H}}$ is a set of valid goals, such that $\mathcal{P}$ is a $\Delta_{\omega_{t}}^{\mathcal{H}}$-deterministic $C H R$ program for input $\mathcal{V G}$. The machine takes an input query $\mathbb{G} \in \mathcal{V G}$ and executes a derivation $\left.d \in \Delta_{\omega_{t}}^{\mathcal{H}}\right|_{\mathbb{G}}$.

To fully specify a CHR machine, we need to give the host language, the CHR program it uses, and the set of queries that are allowed as input. Note that the above definition heavily restricts the class of allowed CHR programs: they have to be $\Delta_{\omega_{t}}^{\mathcal{H}}$-deterministic for valid input queries.

(1) In Chapter 12 we will consider more general types of CHR machines. For now, the above definition suffices and it is considerably simpler. In the terminology of Chapter 12, CHR machines as defined above are both deterministic and abstract (in the sense that they follow the abstract operational semantics $\omega_{t}$ ).

Terminology. Since we only allow deterministic CHR programs, we will assume that there is exactly one derivation corresponding to every goal. If there are different derivations, we arbitrarily pick one - the resulting final state has to be unique anyway, and in case it is successful, there cannot even be different derivation lengths. Therefore we will often talk about the derivation for a given goal as a shorthand for "an arbitrary derivation". We use $\operatorname{deriv}_{\mathcal{M}}(\mathbb{G})$ to denote the derivation for goal $\mathbb{G}$. If the derivation $d=\operatorname{deriv}_{\mathcal{M}}(\mathbb{G})$ is finite, we say the machine terminates for goal $\mathbb{G}$, with output state $\mathcal{M}(\mathbb{G})=\left\langle\mathbb{G}^{\prime}, \mathbb{S}, \mathbb{B}, \mathbb{T}\right\rangle_{n}$ which is the last state of $d$. The machine accepts the input $\mathbb{G}$ if $d$ is a successful derivation and rejects $\mathbb{G}$ if $d$ is a failed derivation. If $d$ is an infinite derivation, we say the machine does not terminate.

A $C H R(X)$ machine is a CHR machine with host language $X$, i.e. of the form $\left(X,,_{-},\right)$. We use $\Phi$ to denote no host language: the built-in constraint theory $\mathcal{D}_{\Phi}$ defines only the basic constraints true and fail, and syntactic equality and inequality (only to be used as an ask-constraint). This implies that the Solve transition can only be used once (to add fail). The only data types are constants, and variables that cannot be bound. A $C H R$-only machine is a $\mathrm{CHR}(\Phi)$ machine.

A sufficiently strong host language $\mathcal{H}$ is a host language whose built-in constraint theory $\mathcal{D}_{\mathcal{H}}$ defines at least true, fail, $==$ and $\backslash==$, the integer numbers and the arithmetic operations for addition, subtraction, multiplication and integer division. Clearly, most realistic host languages are sufficiently strong. Prolog, for instance, defines true, fail, == and $\backslash==$, and allows integer arithmetic using is $/ 2$. 
Listing 10.1: TMSIM: Turing machine simulator

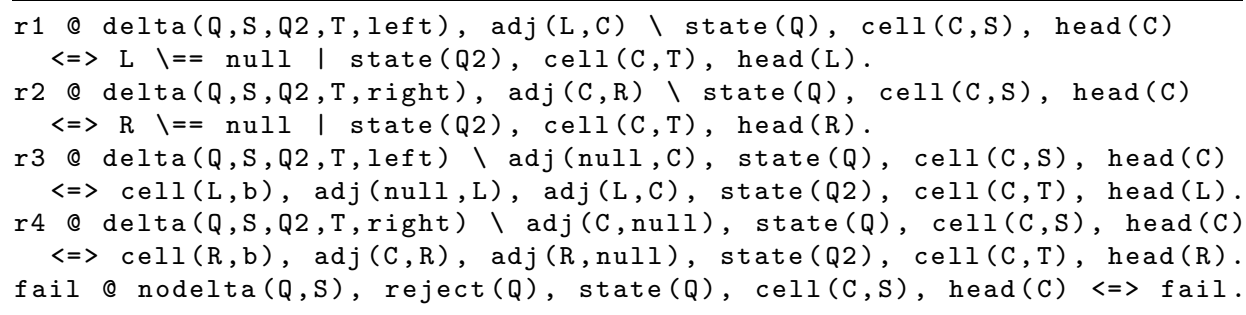

\subsection{CHR machines are Turing-complete}

We now show that CHR-only machines are Turing-complete (cf. Section 2.2.2), i.e. they have at least the computational power of Turing machines. In order to prove this, it suffices to construct a CHR machine which corresponds to a universal Turing machine, i.e. a CHR machine which can simulate any Turing machine.

Consider the CHR program TMSIM shown in Listing 10.1 and the corresponding CHR-only machine $\mathcal{M}_{\mathrm{TM}}=(\Phi, \mathrm{TMSIM}, \mathcal{V} \mathbb{M} \mathcal{G})$. We postpone the definition of $\mathcal{V} \mathbb{M}$ for now. The program TMSIM simulates Turing machines (see Section 2.1.1).

Description. The TMSIM program defines the following constraints:

delta $/ 5$ encodes the transition function $\delta$ (the Turing machine program) in the obvious way: $\operatorname{delta}\left(q, s, q^{\prime}, s^{\prime}, d\right)$ means that $\delta(q, s)=\left(q^{\prime}, s^{\prime}, d\right)$;

nodelta/2 encodes the domain on which $\delta$ is undefined;

reject/1 encodes the set of non-accepting final states $Q \backslash F$;

state/1 contains the current state;

head/1 contains the identifier of the cell under the head;

cell/2 represents a tape cell. The first argument is the unique identifier of the cell. The second argument is the symbol in the cell.

$\operatorname{adj} / 2$ encodes the order of the tape cells. The constraint $\operatorname{adj}(A, B)$ should be read: "the right neighbor of the tape cell with identifier $A$ is the tape cell with identifier $B$ ".

The special cell identifier null is used to refer to a not yet instantiated cell. The rules $r_{3}$ and $r_{4}$ take care of extending the tape as needed. The first four rules of TMSIM correspond to the four Turing machine transition rules. 
A simulation of the execution of a Turing machine $M$ proceeds as follows. The tape input is encoded as cell $/ 2$ constraints and adj/2 constraints. The identifier of the cell to the left of the left-most input symbol is set to null and similarly for the cell to the right of the right-most input symbol. The transition function $\delta$ of $M$ is encoded in multiple delta/5 constraints. All these constraints are combined in the initial query together with the constraint state $\left(q_{0}\right)$ where $q_{0}$ is the initial state of $M$ and the constraint head $\left(c_{1}\right)$ where $c_{1}$ is the identifier of the cell representing the left-most input symbol. Every rule application of the first four rules of TMSIM corresponds directly to a Turing machine transition.

If no more (Turing machine) transitions can be made, the last rule is applicable if the current state is non-accepting. In that case, the built-in constraint fail is added, which leads to a failure state. If the Turing machine ends in an accepting final state, the CHR program ends in a successful final state.

Correctness proof. We define a function tm_to_chr which produces a query for TMSIM, given a Turing machine and an input tape. It is defined as follows: given a Turing machine $M=\left\langle Q, \Sigma, q_{0}, b, F, \delta\right\rangle$ and an input tape $I=\left[i_{1}, \ldots, i_{n}\right]$,

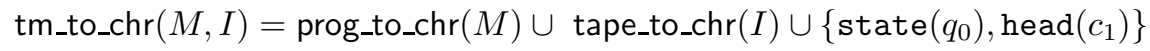

where prog_to_chr $(M)$ is defined as follows:

$$
\begin{aligned}
\operatorname{prog} \text { _to_chr }(M)= & \left\{\operatorname{delta}\left(q, s, q^{\prime}, s^{\prime}, d\right) \mid(q, s) \in Q \times \Sigma \text { and } \delta(q, s)=\left(q^{\prime}, s^{\prime}, d\right)\right\} \\
& \cup\{\operatorname{nodelta}(q, s) \mid(q, s) \in Q \times \Sigma \text { and } \delta(q, s) \text { is undefined }\} \\
& \cup\{\operatorname{reject}(q) \mid q \in Q \backslash F\}
\end{aligned}
$$

and tape_to_chr $(I)$ is defined as follows:

$$
\text { tape_to_chr }(I)=\left\{\operatorname{adj}\left(\operatorname{null}, c_{1}\right)\right\} \cup \bigcup_{j=1}^{n}\left\{\operatorname{cell}\left(c_{j}, i_{j}\right), \operatorname{adj}\left(c_{j}, c_{j+1}\right)\right\}
$$

where $c_{n+1}=$ null and the other $c_{j}$ are unique cell identifiers. Clearly, tm_to_chr can be computed in time linear in the size of the input tape plus the size of the domain of the Turing machine program. We now define $\mathcal{V} \mathbb{M} \mathcal{G}$ as follows:

$\mathcal{V} \mathbb{M} G=\left\{\operatorname{tm} \_\right.$to_chr $(M, I) \mid M$ is a Turing machine for which $I$ is an input tape $\}$.

Lemma 10.2 $\mathcal{M}_{\mathrm{TM}}$ is indeed a CHR machine, that is, the CHR program TMSIM is $\Delta_{\omega_{t}}^{\Phi}$-deterministic for input queries from $\mathcal{V} \mathbb{M} \mathcal{G}$.

Proof. Clearly, the rules of TMSIM maintain a valid tape representation and the invariant that there is at most one head/1 constraint and one state/ 1 constraint. For deterministic Turing machines the first two arguments of delta/ 5 functionally determine the other three arguments. Hence, since valid input corresponds to a 


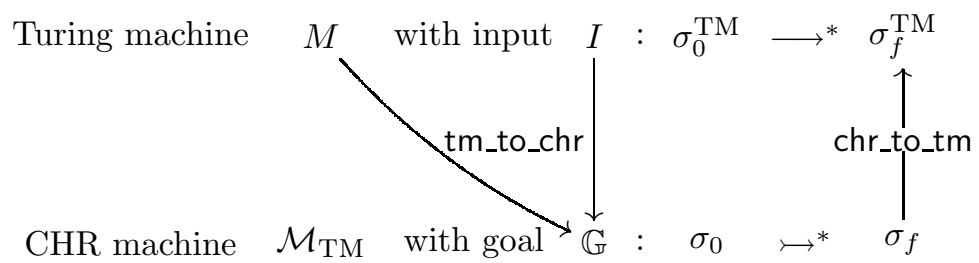

Figure 10.1: Simulation of Turing machines on a CHR machine

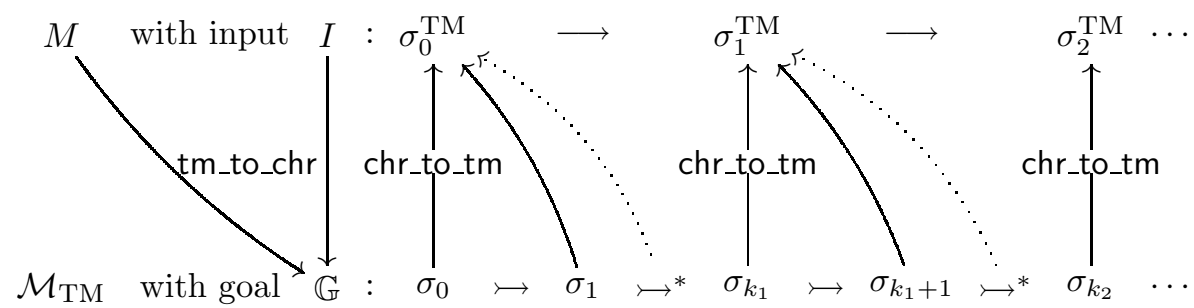

Figure 10.2: States of $\mathcal{M}_{\mathrm{TM}}$ and corresponding Turing machine states

deterministic Turing machine, the rules of TMSIM are mutually exclusive. As a result, the Apply transitions of a derivation are determined, and only the order of the Introduce transitions may vary. However, it can easily be verified that the order of Introduce transitions cannot affect the derivation result and it can only affect the derivation length for failing derivations.

We define a function chr_to_tm which returns a Turing machine execution state, given an execution state for a CHR machine: chr_to_tm : $\Sigma^{\mathrm{CHR}} \rightarrow \Sigma^{\mathrm{TM}}$ :

chr_to_tm $\left(\langle\mathbb{G}, \mathbb{S}, \mathbb{B}, \mathbb{T}\rangle_{n}\right)=\langle q, P, s, N\rangle \quad$ where $q, P, S$, and $N$ are such that $\mathbb{G} \uplus \mathbb{S}=$ prog_to_chr $(M) \cup\{\operatorname{state}(q), \operatorname{head}(c), \operatorname{cell}(c, s)\} \cup \operatorname{tape\_ to\_ chr}(\operatorname{tape}(\langle q, P, s, N\rangle))$.

It can easily be verified that this is indeed a function, and that it can be computed in time linear in the tape size.

We now show that $\mathcal{M}_{\mathrm{TM}}$ can simulate every Turing machine (cf. Figure 10.1).

Theorem 10.3 For any Turing machine $M$ and input tape I :

1. $M$ terminates on input $I \Leftrightarrow \mathcal{M}_{\mathrm{TM}}$ terminates on input tm_to_chr $(M, I)$

2. $M$ accepts $I \Leftrightarrow \mathcal{M}_{\mathrm{TM}}$ accepts tm_to_chr $(M, I)$

3. $M$ outputs chr_to_tm $(X)$ on input $I$ $\Longleftrightarrow \mathcal{M}_{\mathrm{TM}}$ outputs $X$ on input tm_to_chr $(M, I)$ 
Proof. Let $\sigma_{0}=$ initstate(tm_to_chr $\left.(M, I)\right)$ be the initial state of $\mathcal{M}_{\mathrm{TM}}$ with input tm_to_chr $(M, I)$. Note that chr_to_tm $\left(\sigma_{0}\right)=\left\langle q_{0},[], i_{1},\left[i_{2}, \ldots, i_{n}\right]\right\rangle$ is the initial execution state of $M$. We observe the following:

Observation 1. For every $\mathcal{M}_{\mathrm{TM}}$ transition $\sigma_{i} \longmapsto \sigma_{i+1}$, either

(a) chr_to_tm $\left(\sigma_{i}\right) \rightarrow$ chr_to_tm $\left(\sigma_{i+1}\right)$ is a TM transition for $M$, or

(b) chr_to_tm $\left(\sigma_{i}\right)=$ chr_to_tm $\left(\sigma_{i+1}\right)$.

This can be shown by induction on the number of CHR machine steps and case analysis on the transition rules of $\omega_{t}$. The first case (a) holds if $\sigma_{i} \longmapsto \sigma_{i+1}$ by the Apply transition rule, where the applied rule is $\mathrm{r} x$. The second case (b) holds if $\sigma_{i} \longmapsto \sigma_{i+1}$ by the Introduce transition rule. Case (b) also holds when $\sigma_{i} \longmapsto \sigma_{i+1}$ by the Apply transition rule for the rule fail, which can only be followed by (a series of Introduce transitions followed by) a Solve transition which results in a failure state. The relation between the Turing machine derivation steps and the CHR machine steps is shown schematically in Figure 10.2.

Observation 2. If $\sigma_{i}$ is a final state (for $\mathcal{M}_{\mathrm{TM}}$ ), then $\operatorname{chr}$ _to_tm $\left(\sigma_{i}\right)$ is a final state (for $M)$. Furthermore, if $\sigma_{i}$ is a successful final state, then chr_to_tm $\left(\sigma_{i}\right)$ is an accepting final state, and if $\sigma_{i}$ is a failure state, then chr_to_tm $\left(\sigma_{i}\right)$ is non-accepting.

Observation 3. The sub-derivations corresponding to one Turing machine step are finite. In other words, all chains of the form chr_to_tm $\left(\sigma_{n}\right)=\operatorname{chr} \_t$ to_tm $\left(\sigma_{n+1}\right)=$ chr_to_tm $\left(\sigma_{n+2}\right)=\ldots$ have a finite length. This follows from observation 1 and the definition of the Introduce transition, which decrements the size of the (finite) multiset representing the goal in CHR execution states.

Observation 4. If $M$ terminates on input $I$, then the $\mathcal{M}_{\mathrm{TM}}$ derivation on input tm_to_chr $(M, I)$ is also finite. This follows from observation 1 and 3 .

The three properties follow straightforwardly from the above observations.

\subsection{Turing-complete subsets of CHR}

The TMSIM program of Listing 10.1 contains only simpagation and simplification rules. It can easily be modified to consist only of simplification rules: the kept part of the simpagation rules can be removed and reinserted in the body. Similarly, it can easily be modified to contain only simpagation rules. So clearly, CHR with simplification rules only, or simpagation rules only, is Turing-complete.

In this section we identify some other subsets of CHR that are also Turingcomplete. We do this by programming a Turing machine simulator under the corresponding restrictions of CHR.

\subsubsection{Only propagation rules}

It is possible to simulate Turing machines in CHR with only propagation rules. Since constraints cannot be destructively updated, we add a time-stamp argument 
Listing 10.2: TMSIM-PROP: Turing machine simulator, only propagation rules

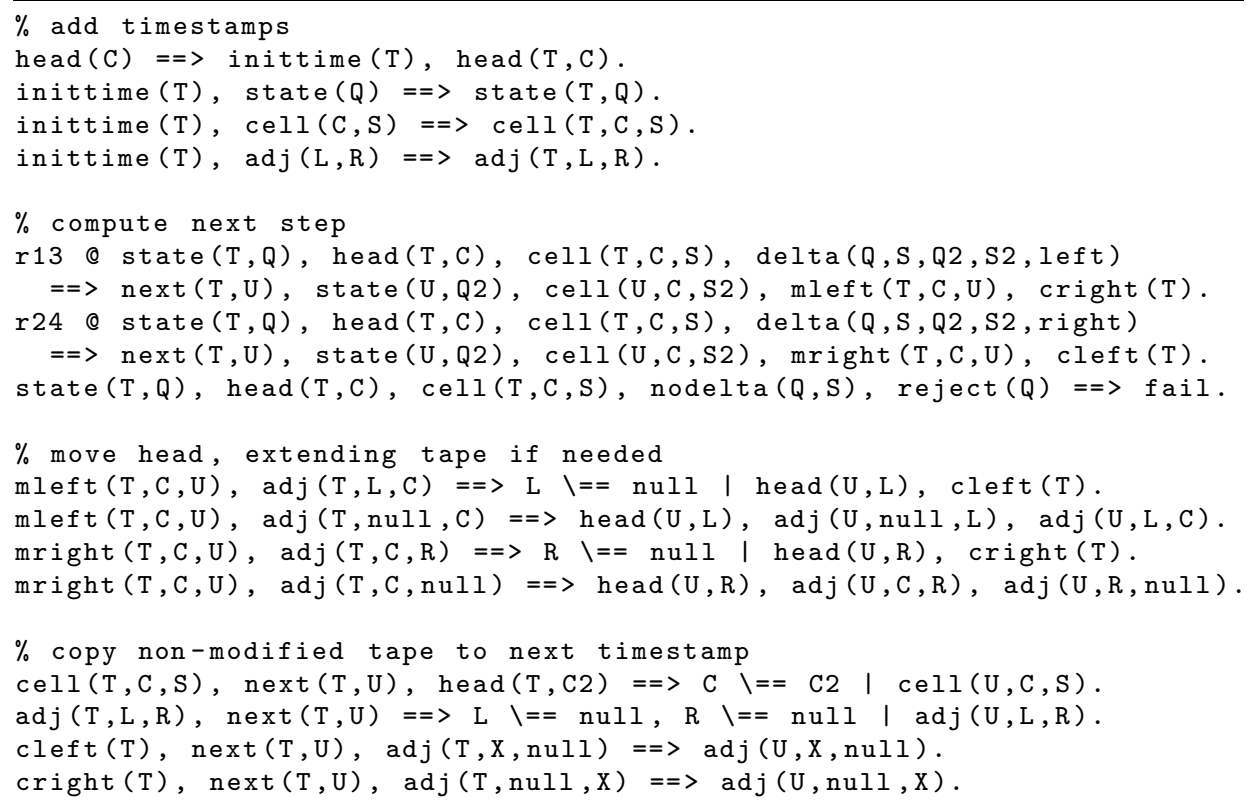

to all constraints that are updated. By carefully making sure that the representation of the part of the Turing machine tape that was not modified is copied from one time-stamp to the next, while the modified constraints are not copied, the correct behavior can be obtained without destructive updates. Listing 10.2 shows the modified program TMSIM-PROP. This shows that CHR with only propagation rules is also still Turing-complete.

Since the entire tape is copied at every step, the space used by this program is $O(T S)$ when simulating a $T$-time, $S$-space Turing machine. This is considerably less efficient than the $O(S)$ space usage of the TMSIM program. Some space overhead is inevitable when using only propagation rules: since no constraints can ever be removed, at least space usage linear in the number of updated cells is needed, i.e. $O(T)$ space usage. Whether this lower bound can be achieved is not clear.

\subsubsection{No rule guards}

The above Turing machine simulator programs all require the use of a rule guard to distinguish the normal case (new position is not null) and the case in which the tape representation needs to be extended. The explicit use of rule guards can be 
Listing 10.3: TMSIM-NG: Turing machine simulator, without rule guards

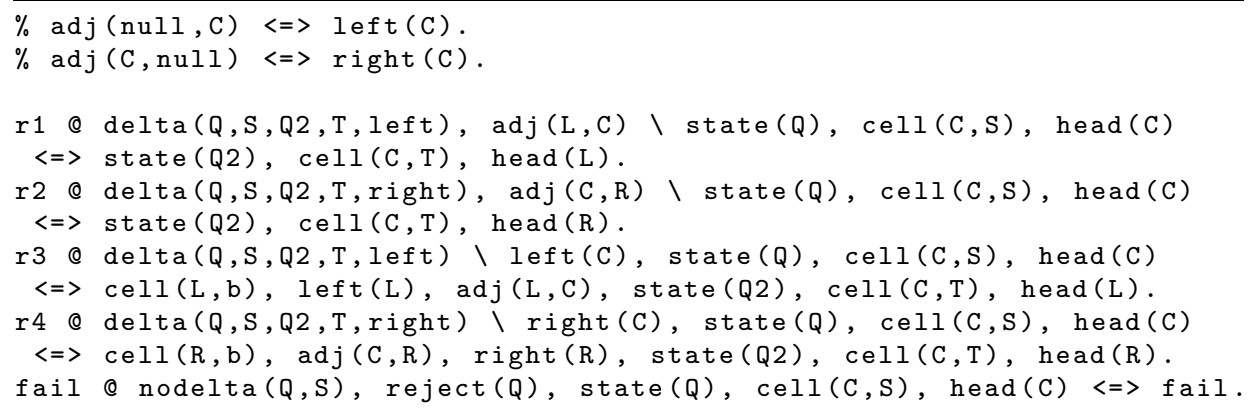

avoided by using a slightly different representation: instead of using adj (null, C) to denote that the cell position $\mathrm{C}$ is the left-most cell of the current tape representation, we now use left(C). Similarly we replace adj (C,null) by right(C). In this way, the explicit disequality guard in the TMSIM program can be avoided. Listing 10.3 gives the resulting program TMSIM-NG.

\subsubsection{Only one rule}

Now let us see how many rules are really needed. First of all, the rule fail is not that important. The reason is that instead of considering the accept/reject behavior of a Turing machine as the 'output' of the computation on a given string, we can equivalently consider the final tape content as the output. Every Turing machine program that accepts certain strings and rejects others can easily be rewritten to a program that clears the tape and writes a single " 1 " for accepted strings and a single "0" for rejected strings, and always stops in an accepting final state. So without loss of generality, we may assume that we can drop the last rule. That still leaves us with four rules. For each direction of tape movement (left and right), we have two rules: one for the normal case and one for the case in which the tape representation needs to be extended. Using a slightly different tape representation, these two cases can be merged. In the resulting rules, no rule guard is needed because there is no need to distinguish two cases - both cases are handled by the same rule.

The idea is as follows. The tape is still represented using adjacency constraints $\operatorname{adj} / 2$, but at the tape ends, instead of pointing to null, we put a loop at both ends, as shown in Figure 10.3. We add a blank cell at each end, and let it point to itself. The rectangles indicate tape cells and the adj $/ 2$ constraints are represented by arrows. Now, suppose we have to move to the right (the case for moving to the left is symmetric). We create a new cell and add two adj $/ 2$ constraints as shown in Figure 10.4. We overwrite the current cell symbol with the blank symbol b 


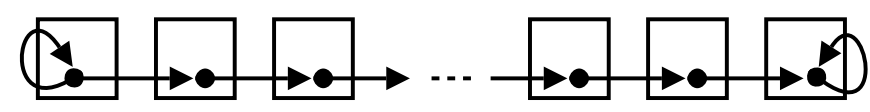

Figure 10.3: Tape representation for TMSIM-2R

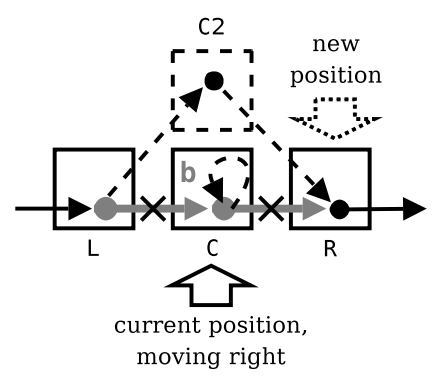

Figure 10.4: Right move in TMSIM-2R, normal case

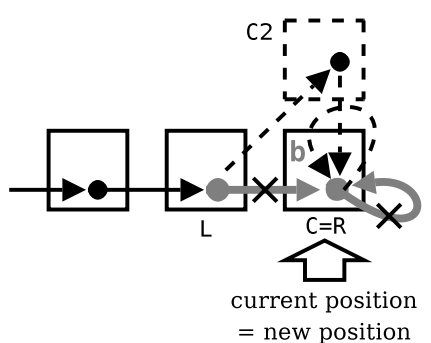

Figure 10.5: Right move in TMSIM-2R, at the tape end

Listing 10.4: TMSIM-2R: Turing machine simulator in two rules

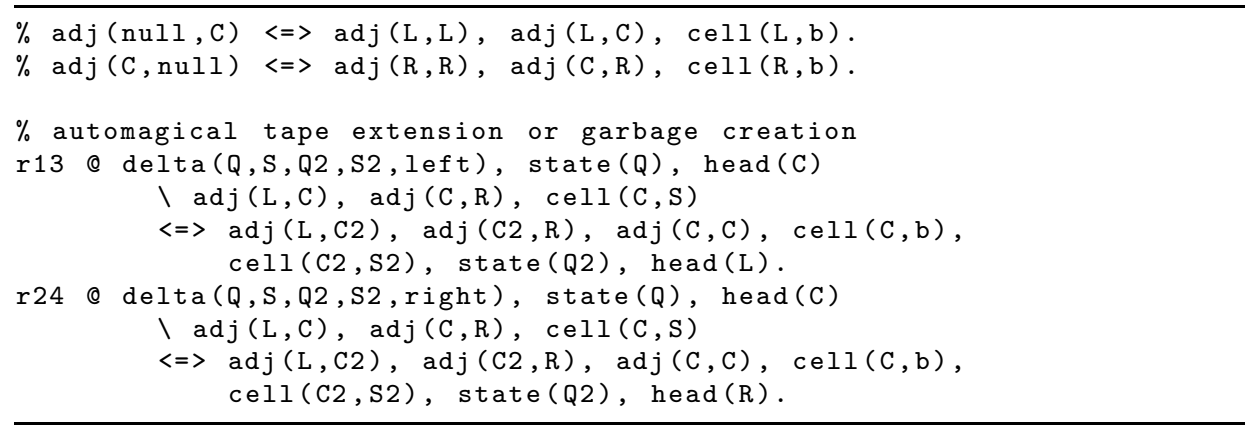


Listing 10.5: TMSIM-2RD: A version of TMSIM-2R with double adj/3 constraints

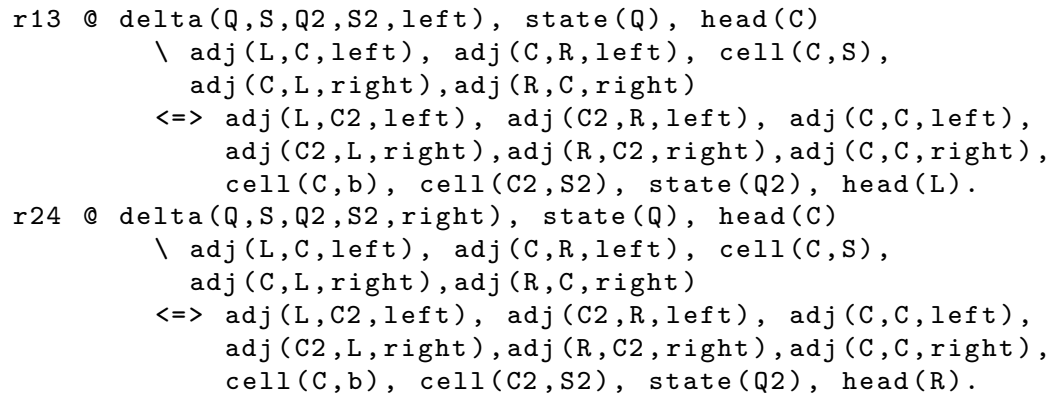

and remove the incoming and outgoing adj $/ 2$ constraints. As a result, the current cell becomes isolated from the rest of the tape. Finally we add a looping adj $/ 2$ constraint at the current cell.

In the normal case (where we are not at the tape end), the net result is that the current cell becomes isolated and a tape 'bypass' is created. Figure 10.5 shows what happens when we move to the right near the right tape end. It is easy to see that the net result is that the tape has been extended with one cell, and we are again at the tape end. In Listing 10.4 the resulting CHR program TMSIM-2R is shown; it has only two rules. The two rules that are commented out show how to change the old tape representation to the new form, but we assume that the query is already in the correct form.

Now can we further reduce this program? Certainly! It is easy to see that there is a lot of symmetry between the two rules in TMSIM-2R. We can change the tape representation again to take advantage of this symmetry and merge the rules. Instead of using adj (A,B) to represent that cell $A$ is to the left of cell $B$, we now use the following redundant representation: $\operatorname{adj}(A, B$, left $), \operatorname{adj}(B, A, \operatorname{right})$. So $\operatorname{adj}(A, B, X)$ means that cell $A$ is to the $X$ of cell $B$. By replacing every $\operatorname{adj} / 2$ constraint in the two-rule program TMSIM-2R by the corresponding two adj $/ 3$ constraints, we get the program TMSIM-2RD of Listing 10.5. If we now connect the third argument of the adj/3 and the last argument of the delta/5 constraint, both rules in TMSIM-2RD become a variable renaming of each other. Clearly, one of these rules suffices. Listing 10.6 shows the resulting CHR program TMSIM-1R.

\subsubsection{Only single-headed rules}

The program TMSIM-1R consists of only one rule, but that rule has 8 head constraints. All rules with more than two heads can be reduced to several rules with only two heads by explicitly constructing partial joins. However, as we shall see shortly, single-headed rules only do not suffice. 
Listing 10.6: TMSIM-1R: Turing machine simulator in one rule

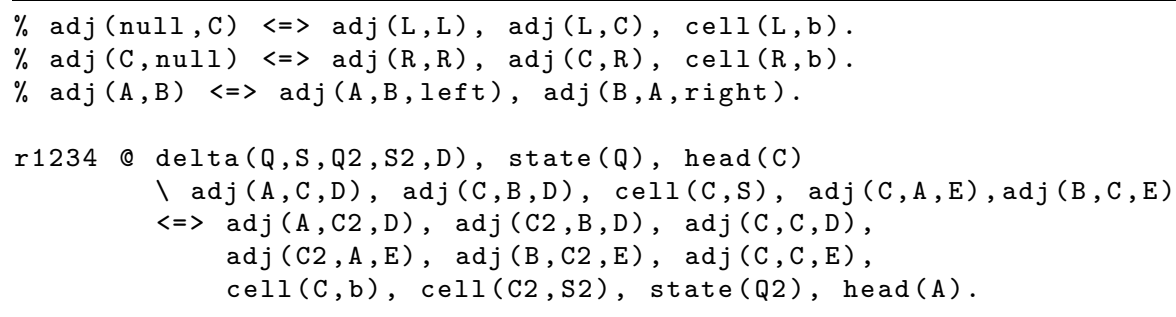

Listing 10.7: MINSKY-A: Minsky machine encoding using argument arithmetic

\begin{tabular}{|c|c|c|c|}
\hline$\llbracket p_{i}: \operatorname{succ}\left(r_{1}\right) \rrbracket$ & $=$ & $\mathrm{mm}\left(p_{i}, \mathrm{~A}, \mathrm{~B}\right)$ & $\Leftrightarrow \mathrm{mm}\left(p_{i+1}, \mathrm{~A}+1, \mathrm{~B}\right)$. \\
\hline$\llbracket p_{i}: \operatorname{succ}\left(r_{2}\right) \rrbracket$ & $=$ & $\mathrm{mm}\left(p_{i}, \mathrm{~A}, \mathrm{~B}\right)$ & $\Leftrightarrow \mathrm{mm}\left(p_{i+1}, \mathrm{~A}, \mathrm{~B}\right.$ \\
\hline$\llbracket p_{i}: \operatorname{decjump}\left(r_{1}, p_{j}\right) \rrbracket$ & $=$ & $\left\{\begin{array}{l}\mathrm{mm}\left(p_{i}, \mathrm{~A}, \mathrm{~B}\right) \\
\mathrm{mm}\left(p_{i}, \mathrm{~A}, \mathrm{~B}\right)\end{array}\right.$ & $\begin{array}{ll:l}\Leftrightarrow A & \mathrm{~A}=0 & \mathrm{~mm} \\
\Leftrightarrow & \left(p_{j}, \mathrm{~A}, \mathrm{~B}\right) . \\
\Leftrightarrow A & >0 & \mathrm{~mm}\left(p_{i}, \mathrm{~A}-1\right.\end{array}$ \\
\hline$\llbracket p_{i}:$ decj & $=$ & $\left\{\begin{array}{l}\mathrm{mm}\left(p_{i}, \mathrm{~A}, \mathrm{~B}\right) \\
\mathrm{mm}\left(p_{i}, \mathrm{~A}, \mathrm{~B}\right)\end{array}\right.$ & \begin{tabular}{l|l|l}
$\Leftrightarrow \mathrm{B}=0$ & $\mathrm{~mm}\left(p_{j}, \mathrm{~A}, \mathrm{~B}\right)$. \\
$\Leftrightarrow$ & $\mathrm{B}>0$ & $\left.\mathrm{~mm}\left(p_{i+1}, \mathrm{~A}, \mathrm{~B}-1\right).\right\}$
\end{tabular} \\
\hline
\end{tabular}

All of the above programs were $\operatorname{CHR}(\Phi)$ programs: they do not depend on advanced host language data types or built-ins. Their rules use only syntactic (dis)equality tests in their (implicit) guards, and the constraint arguments are only constants and variables. An arbitrary number of fresh variables can be created if the body of a rule contains variables that do not occur in the head, but all variables are syntactically different objects and they cannot be bound to each other or to constants (as in Prolog). $\operatorname{CHR}(X)$ with only single-headed rules can be shown to be Turing-complete for a sufficiently strong host language $X$. However, singleheaded CHR-only is not Turing-complete.

We first show that with the help of the host language, CHR with only singleheaded rules is still Turing-complete. In the above programs we have programmed a Turing machine simulator in CHR, but it would also be sufficient to give a method to encode any given Turing machine - in particular, a universal Turing machine - as a CHR program. We will now give a method to encode any Minsky machine (see Section 2.1.4) in single-headed CHR; since Minsky machines are Turing-equivalent, this also demonstrates the Turing-completeness of singleheaded CHR. We encode every Minsky machine instruction $p_{i}$ : instr as one or a few CHR rules $\llbracket p_{i}$ : instr $\rrbracket$; the encoding of a full program is simply the union of all the encoded rules. Listing 10.7 shows one way to do this encoding, due to Di Giusto et al. (2008). Assuming that both registers are initialized to zero and the first program line is called $\mathrm{p} 1$, the initial query is $\mathrm{mm}(\mathrm{p} 1,0,0)$.

Alternatively, successor notation can be used, so an argument of the form 
Listing 10.8: MINSKY-C: Minsky machine encoding using constraint chains

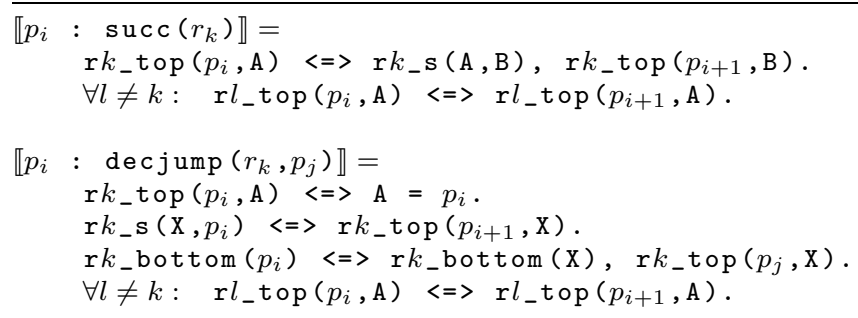

$\mathrm{s}(\mathrm{s}(\mathrm{s}(\ldots \mathrm{s}($ zero $) \ldots \mathrm{s}))$, with $n$ times $\mathrm{s}$, indicates the number $n$. In both cases however, a nontrivial host language data type is needed - respectively integers and complex terms. Therefore, in some sense, an essential part of the computation is done in the host language and not in CHR itself.

Listing 10.8 shows another way to encode Minsky machines in single-headed CHR rules. Only the basic data types of $\mathrm{CHR}(\Phi)$ are used: atoms and variables. The value of each register is represented as a chain of successor constraints. For example, if register $r_{1}$ has the value 3 , it would be encoded as follows:

$r 1$ bottom $(A), r 1$ s $(A, B), r 1 \_s(B, C), r 1 \_s(C, D), r 1$ top $(p, D)$

where $\mathrm{p}$ is the current program line. Assuming that both registers are initialized to zero and the first program line is called p1, the initial query would be

r1_bottom(A), r1_top (p1,A), r2_bottom(B), r2_top (p1,B)

In the program MINSKY-A, there is always only one constraint, and its arguments can grow arbitrarily large. In contrast, in the program MINSKY-C, the number of constraints can grow arbitrarily large, but each constraint has fixedsize arguments. The use of host language features is obvious in MINSKY-A: host language arithmetic is used in every rule. In the program MINSKY-C, the use of host language features is a bit more subtle: the only host language 'trick' that it uses is the unification in the first rule for decjump instructions. This unification is crucial since it provides the mechanism that allows 'communication' to link different constraints in the constraint store. Using only single-headed rules, there is no way to connect the constraints in the store except via the host language. This is fundamentally different in multi-headed rules, where constraints in the store can be linked using shared arguments in the head of a rule. For example, using two-headed rules, we can modify MINSKY-C to encode an instruction of the form $p_{i}$ : decjump $\left(r_{1}, p_{j}\right)$ without needing host language built-ins:

r1_bottom $(\mathrm{A}) \backslash \mathrm{r} 1 \_t o p\left(p_{i}, \mathrm{~A}\right) \Leftrightarrow \mathrm{r} 1 \_t o p\left(p_{j}, \mathrm{~A}\right)$.

r1_s $(\mathrm{A}, \mathrm{B}), \operatorname{r1\_ top}\left(p_{i}, \mathrm{~B}\right) \Leftrightarrow$ r1_top $\left(p_{i+1}, \mathrm{~A}\right)$. 
Listing 10.9: MINSKY-P: Minsky machine encoding in propositional refined CHR

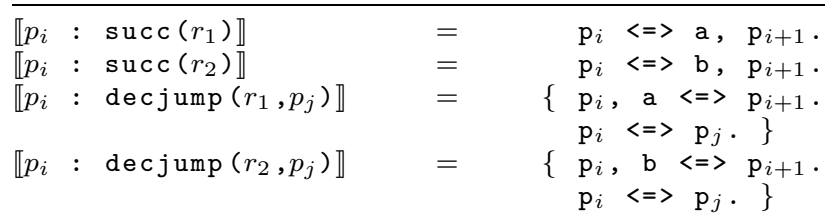

\subsubsection{Propositional CHR}

In fact, without a host language, CHR with only single headed rules is a very weak formalism - it is not more powerful than propositional CHR-only:

Lemma 10.4 $C H R(\Phi)$ with only single-headed rules can be reduced to propositional $C H R(\Phi)$, i.e. CHR with only zero-arity constraints.

Proof (sketch). Assume there are $k$ distinct constants used in the program and queries. For every CHR constraint $c / n$, we make 'flattened' versions of it, one for every possible combination of arguments. Since variables cannot be bound and since in single-headed rules there can only be matching between the arguments of a single constraint, it does not matter what the variables are, but only what variables are shared between the arguments. Hence, there are less than $(k+n)^{n}$ different combinations of arguments for a constraint of arity $n$. We replace the body CHR constraints by their flattened version, and duplicate the rule for every matching flattened version of the head. The resulting program is propositional.

We have the following negative result for propositional CHR-only:

Theorem 10.5 Propositional $C H R(\Phi)$ is not Turing-complete.

Proof (sketch). Propositional CHR $(\Phi)$ programs can easily be encoded as placetransition Petri nets — see Section 3.7.4 and (Betz 2007). Since the latter is not Turing-complete (Jantzen and Valk 1979), neither is the former.

The above lemma and theorem can be combined in the obvious way:

Corollary 10.6 $\mathrm{CHR}(\Phi)$ with only single-headed rules is not Turing-complete.

Somewhat surprisingly, Theorem 10.5 only holds when the full abstract semantics $\omega_{t}$ is considered (as we implicitly do in this chapter, cf. Chapter 12). If the refined operational semantics $\omega_{r}$ can be used, the result no longer holds. The reason is that the $\omega_{r}$ semantics allows checking for the absence of a constraint (cf. negation as absence, Section 3.4.2), which is not possible in the abstract semantics. Listing 10.9 shows an encoding of Minsky machines in propositional CHR. It 
crucially depends on the refined semantics. The value of register $r_{1}$ is represented by the multiplicity of the constraint a, and the value of $r_{2}$ by the multiplicity of b. Under the abstract semantics this program breaks down because the second rule for decjump instructions can be fired even if the corresponding register does not have the value zero - this cannot happen in the refined semantics because of the rule order. Thus, propositional CHR is not Turing-complete in the abstract semantics, but it is Turing-complete in the refined semantics. Propositional CHR in the priority semantics $\omega_{p}$ is also Turing-complete: it suffices to assign a higher priority to the first rule for decjump than to the second rule. We conjecture that single-headed $\operatorname{CHR}(\Phi)$ remains Turing incomplete even under the $\omega_{r}$ or $\omega_{p}$ semantics.

\subsubsection{Summary}

We have demonstrated that many subsets of CHR are still Turing-complete. CHR with only one kind of rules (simplification, propagation, or simpagation) is still Turing-complete. Explicit rule guards are not needed. Multiple rules are not needed - one single rule suffices. If a (sufficiently strong) host language is available, single-headed rules suffice.

Some very restricted subsets of CHR turned out to be not Turing-complete. Propositional CHR, in which all constraints have arity zero, is not Turing-complete, so allowing constraints to have arguments (even only non-compound arguments) does add computational power. Single-headed CHR without host language is also not Turing-complete, so computational power is gained by the availability of either multi-headed rules or a host language. Finally, the added execution control of a more instantiated operational semantics (for example the refined semantics or the priority semantics) also adds computational power in the sense that propositional CHR becomes Turing-complete under a more instantiated semantics.

Figure 10.6 gives an overview of our results. The columns correspond to different properties of the rules: one or more rules, single-headed or multi-headed rules, and propagation rules only ("P") or simplification rules only ("S"); simpagation and propagation rules can be written as simplification rules so the "S" column can also be read as "all kinds of rules allowed". The rows correspond to restrictions on the constraint arguments or host language; every row is a subset of the row below. White cells indicate subsets that are not Turing-complete while dark cells indicate Turing-complete subsets. Gray cells with a question mark indicate subsets that we have not discussed; their computability properties need further investigation.

Most of the results in Figure 10.6 follow directly from Theorem 10.5, Corollary 10.6, and the various simulator programs, as indicated. The status of the subsets of CHR that are marked with (a) and (b) requires explanation.

The two subsets marked with (a), refined propositional CHR with only one or multiple propagation rules, are not Turing complete. The reason is that all propositional propagation-only programs are confluent (only the order of rule ap- 


\begin{tabular}{|c|c|c|c|c|c|c|c|c|c|}
\hline & \multicolumn{4}{|c|}{ only one rule } & \multicolumn{4}{|c|}{ multiple rules } \\
\hline & & \multicolumn{2}{|c|}{$\begin{array}{l}\text { single- } \\
\text { headed }\end{array}$} & \multicolumn{2}{|c|}{$\begin{array}{l}\text { multi- } \\
\text { headed }\end{array}$} & \multicolumn{2}{|c|}{$\begin{array}{l}\text { single- } \\
\text { headed }\end{array}$} & \multicolumn{2}{|c|}{$\begin{array}{l}\text { multi- } \\
\text { headed }\end{array}$} \\
\hline & & $\mathrm{P}$ & S & $\mathrm{P}$ & S & $\mathrm{P}$ & S & $\mathrm{P}$ & S \\
\hline \multirow{2}{*}{$\begin{array}{c}\text { propositional } \\
\text { CHR } \\
\text { (no arguments) }\end{array}$} & abstract & & & & & & Th & orem & 10.5 \\
\hline & refined & & & (a) & ? & & & (a) & MINSKY \\
\hline \multicolumn{2}{|c|}{ CHR-only } & Cor & 10.6 & $?$ & $\begin{array}{l}\text { TMSIM } \\
-1 R\end{array}$ & Cor. & 10.6 & $\begin{array}{l}\text { TMSIM } \\
\text {-PROP }\end{array}$ & TMSIM \\
\hline \multicolumn{2}{|c|}{$\begin{array}{l}\text { CHR with sufficiently } \\
\text { strong host language }\end{array}$} & ? & $?$ & $?$ & & (b) & $\begin{array}{c}\text { MINSK } \\
-A\end{array}$ & & \\
\hline \multicolumn{2}{|c|}{$\begin{array}{c}\text { CHR with Turing-complete } \\
\text { host language }\end{array}$} & & & & & & & & \\
\hline
\end{tabular}

Figure 10.6: Overview of subsets of CHR and their Turing-completeness

plication can be chosen, so if rules are applied exhaustively, the end result is always the same); this means that if a propositional propagation-only program exists that simulates a universal Turing machine under the refined semantics, it must be confluent and thus it also works under the abstract semantics, which is impossible because of Theorem 10.5.

Finally, the subset marked with (b), single-headed propagation-only CHR with a sufficiently strong host language, is Turing complete. To see this, consider the program MINSKY-A and replace all simplification arrows ('<=>') with propagation arrows ('==>'). This new program behaves exactly like the old one, except that it keeps a track of the entire computation. To read the output of the simulated Minsky machine, it suffices to look at the most recently added constraint (the one with the highest identifier).

(-)) Alternatively (say, if one considers inspecting identifiers a form of cheating), an explicit halt instruction can be added to the Minsky machine, which is to be translated to a rule of the form "mm $\left(p_{i}, \mathrm{~A}, \mathrm{~B}\right)==>\operatorname{output}(\mathrm{A}, \mathrm{B})$ ". 


\subsection{Complexity of CHR machines}

Thus far we have only considered the computability of CHR machines (and various restrictions of $\mathrm{CHR}$ ). In the following chapters we will investigate the computational complexity of CHR machines. But first, we need to complete the definition of CHR machines of Section 10.1, and define what exactly we mean with the time and space complexity of a CHR machine.

\subsubsection{Time complexity}

We will use a rather simple and natural measure for the time complexity of CHR machines:

Definition 10.7 (time function) Given a $C H R$ machine $\mathcal{M}=(\mathcal{H}, \mathcal{P}, \mathcal{V G})$ and a valid goal $\mathbb{G}$, the time function chrtime $\mathcal{M}$ returns the derivation length:

$$
\text { chrtime }_{\mathcal{M}}: \mathcal{L G} \rightarrow \mathbb{N}: \mathbb{G} \mapsto \# \operatorname{deriv}_{\mathcal{M}}(\mathbb{G}) \text {. }
$$

Definition 10.8 (time complexity of CHR machines) Given a CHR machine $\mathcal{M}=(\mathcal{H}, \mathcal{P}, \mathcal{L})$ and assuming that host language constraints of $\mathcal{H}$ take constant time, the (worst-case) time complexity function CHRTIME $_{\mathcal{M}}$ is defined as follows:

$$
\operatorname{CHRTIME}_{\mathcal{M}}(n)=\max \left\{\operatorname{chrtime}_{\mathcal{M}}(\mathbb{G}) \mid \mathbb{G} \in \mathcal{V G} \wedge \text { inputsize }(\mathbb{G})=n\right\}
$$

where inputsize is a function which returns the size of a goal.

The function inputsize can be problem-specific: for instance, depending on the problem at hand, if the input is an integer number $x$ (wrapped in some constraint), the input size function could be defined as the number of bits needed to represent $x$, or just as the number $x$ itself - this choice of course dramatically influences the resulting complexity. From now on, we will assume that the usual term size is used, unless otherwise mentioned.

\footnotetext{
Example 39 (time complexity of PRIMES). Consider the CHR machine $\mathcal{M}_{P}=($ Prolog, PRIMES, $\{\operatorname{upto}(n) \mid n \in \mathbb{N}\})$, where the program PRIMES is that of Listing 3.2 on page 31. An appropriate goal size function is given by inputsize $(\operatorname{upto}(n))=n$. Every derivation starting with upto $(n)$ consists of $n-1$ Solve steps (one for every computation of $\mathrm{N}-1$ ), $2 n-1$ Introduce steps $(n$ upto/ 1 constraints and $n-1$ prime/ 1 constraints), and $n+$ nonprimes $(n)<2 n$ Apply steps, where nonprimes $(n)$ is the number of composite numbers between 2 and $n$ (the loop rule is applied $n-1$ times, the stop rule once, and the absorb rule nonprimes $(n)$ times). As a result, the time complexity $\operatorname{CHRTIME}_{\mathcal{M}_{P}}(n)$ is smaller than $5 n-2$ and thus it is $O(n)$.
} 


\begin{tabular}{|c|c|c|c|c|}
\hline A & B & C & D & E \\
\hline F & & G & & H \\
\hline I & J & K & & L \\
\hline & M & N & O & P \\
\hline Q & R & S & T & U \\
\hline
\end{tabular}

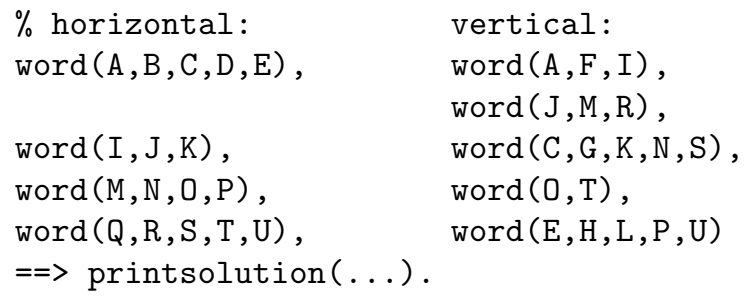

Figure 10.7: Solver program for some crossword puzzle.

The time given by chrtime $_{\mathcal{M}}$ is the time needed by a theoretical CHR machine. The next chapter deals with the relation between this theoretical time and the time needed by practical CHR implementations. The distinction is important because the definition of chrtime $\mathcal{M}$ does not correspond to the reality of CHR implementations, as the following example illustrates (cf. Theorem 11.8).

Example 40 (crossword puzzle). A CHR machine can solve a crossword puzzle in time linear in the number of words. Given a query containing $n$ words from a dictionary as word $/ k$ constraints (word length $k$ ), the CHR machine with the program of Figure 10.7 returns all $s$ solutions for a crossword puzzle in time $O(n+s)$. It seems highly unlikely that this time complexity can be achieved on practical computers (i.e. on a RAM machine) for arbitrary puzzles.

\subsubsection{Space complexity}

We define the size of a CHR machine state as follows:

\section{Definition 10.9 (state size function)}

$$
\operatorname{SIZE}: \Sigma^{\mathrm{CHR}} \rightarrow \mathbb{N}:\langle\mathbb{G}, \mathbb{S}, \mathbb{B}, \mathbb{T}\rangle_{n} \mapsto \operatorname{size}(\mathbb{G})+\operatorname{size}(\mathbb{S})+\operatorname{size}(\mathbb{B})+\operatorname{size}(\mathbb{T})
$$

where for sets $X$, size $(X)=\sum_{x \in X}|x|$ and the size $|x|$ is the usual term size.

The space complexity of a CHR machine is defined in the usual way:

Definition 10.10 (space function) Given a CHR machine $\mathcal{M}=(\mathcal{H}, \mathcal{P}, \mathcal{L G})$ and a valid goal $\mathbb{G}$, the space function chrspace ${ }_{\mathcal{M}}$ returns the largest state size encountered during the derivation for that goal:

$$
\operatorname{chrspace}_{\mathcal{M}}(\mathbb{G})=\max \left\{\operatorname{SIZE}(\sigma) \mid \sigma \in \operatorname{deriv}_{\mathcal{M}}(\mathbb{G})\right\}
$$


Definition 10.11 (space complexity of CHR machines) Given a CHR machine $\mathcal{M}=(\mathcal{H}, \mathcal{P}, \mathcal{V G})$, the space complexity function $\mathrm{CHRSPACE}_{\mathcal{M}}$ is defined as follows:

$$
\operatorname{CHRSPACE}_{\mathcal{M}}(n)=\max \left\{\operatorname{chrspace}_{\mathcal{M}}(\mathbb{G}) \mid \mathbb{G} \in \mathcal{V G} \wedge \text { inputsize }(\mathbb{G})=n\right\}
$$

Example 41 (space complexity of PRIMES). Consider, as in Example 39, the CHR machine $\mathcal{M}_{P}$ and the goal size function inputsize(upto $\left.(n)\right)=n$. If we assume all integers can be represented in a fixed number of bits, the space complexity CHRSPACE $\mathcal{M}_{P}$ is $O(n)$. Note that if the constraint size is bounded, the size of the constraint store is asymptotically dominated by the number of Introduce steps, and the size of the built-in store is dominated by the number of Solve steps. 


\title{
Chapter 11
}

\section{Complexity-wise Completeness}

\begin{abstract}
"You insist that there is something that a machine can't do. If you will tell me precisely what it is that a machine cannot do, then I can always make a machine which will do just that."

— John von Neumann (1903-1957)

"Generally, old media don't die. They just have to grow old gracefully. Guess what, we still have stone masons. They haven't been the primary purveyors of the written word for a while now of course, but they still have a role because you wouldn't want a $T V$ screen on your headstone."
\end{abstract}

— Douglas Adams (1952-2001)

In this chapter we investigate the complexity of CHR machines and their practical implementation in the Leuven CHR system. RAM machines are more realistic and faster than Turing machines, so from the complexity point of view it makes sense to focus on RAM machines. We show how to program the CHR machine to efficiently simulate RAM machines. We then discuss how to simulate CHR machines efficiently on a RAM machine, a problem that corresponds to optimizing compilation of CHR programs. This results in a general complexity meta-theorem. Finally we apply this meta-theorem to the CHR program that simulates RAM machines. This allows us to conclude that existing CHR compilation techniques, in particular those described in Chapter 4 and in Part II of this dissertation, suffice to implement the RAM machine simulator efficiently. As a result, all RAM machine programs (so also every known algorithm) can be translated automatically to CHR programs that have the same complexity. 


\subsection{RAM machine simulators}

We now show that the CHR machine is at least as efficient as the RAM machine. Specifically, the CHR-only machine can simulate any PA-RAM machine with the same time complexity, and if $\mathcal{H}$ is a sufficiently strong host language, then $\operatorname{CHR}(\mathcal{H})$ machines can simulate standard RAM machines with the same time and space complexity.

Theorem 11.1 A CHR-only machine $\mathcal{M}_{\text {PARAM }}$ exists which can simulate, in $O(T+P+I)$ time and $O(P+J)$ space, a T-time, $S$-space Peano-arithmetic $R A M$ machine with a program of $P$ lines, where $I$ is the sum of the values of the input registers and $J$ is the maximal sum of the values of all registers during the entire computation.

Proof. The proof roughly corresponds to that of Theorem 10.3. Consider the CHR-only program PARAMSIM (see Listing A.1), and the corresponding CHRonly machine ( $\Phi$, PARAMSIM, VPARAMG).

The mapping from CHR machine states to RAM machine states is as follows. Memory cells are represented as $\mathrm{m}(A, V)$ constraints, where $A$ is the address and $V$ refers to the value. If $V$ is the atom zero, the value is zero. Otherwise, there is a successor constraint $\mathbf{s}(V, W)$ which expresses that $V=W+1$. Again, $W$ can be either zero or there is another successor constraint $\mathbf{s}(W, X)$, etc. For example, to represent that the register $r_{1}$ contains the value 3 , the following conjunction of constraints could be used: $\mathrm{m}\left(r_{1}, N_{3}\right), \mathrm{s}\left(N_{3}, N_{2}\right), \mathrm{s}\left(N_{2}, N_{1}\right), \mathrm{s}\left(N_{1}\right.$, zero $)$.

The mapping from a RAM machine program and input to an initial CHR goal is now obvious. We define VPARAMG to be the image of this mapping. The RAM machine program is encoded as $i /\{3,4,5\}$ constraints; the first argument represents the label (or line number), the second argument is the label of the next program line, the other arguments represent the actual instruction. The input memory cells are represented as above. The program counter is set to the label of the first line of the program by adding a corresponding initial c/1 constraint.

After the initialization, one rule is applied for every step of the RAM machine. Applying a rule causes at most three constraints to be inserted. Therefore, the number of CHR steps is bounded by four times the number of RAM machine steps, plus $P$ steps to Introduce the $\mathrm{i} /\{3,4,5\}$ constraints. Initializing the input memory cells to their values means inserting one $\mathrm{m} / 2$ constraint for every input and $I \mathrm{~s} / 2$ constraints. The number of registers (and hence $\mathrm{m} / 2$ constraints) is bounded by the program size $P$ (remember we don't have indirection), so the total time complexity of the CHR machine is $O(T+P+I)$. The CHR store contains $P$ i/ $\{3,4,5\}$ constraints; since the number of $\mathrm{m} / 2$ constraints is $O(P)$ and the number of $\mathrm{s} / 2$ constraints is bounded by $J$, the space complexity of the CHR machine is $O(P+J)$. 
The PARAMSIM program does not use any host language arithmetic operations. Its unary number representation causes an exponential space penalty. If host language arithmetic is available, a similar program can be written which uses only $O(P+S)$ space by directly storing the value of a register in the second argument of the $\mathrm{m} / 2$ constraints.

We can also simulate the more realistic standard-arithmetic RAM machine in CHR. However, if we want to do this without a harsh complexity penalty, we need host language support to handle the integers.

Theorem 11.2 For any sufficiently strong host language $\mathcal{H}$, a $C H R(\mathcal{H})$ machine $\mathcal{M}_{\mathrm{RAM}}$ exists which can simulate, in $O(T+P+S)$ time and $O(S+P)$ space, a $T$-time, $S$-space standard RAM machine with a program of $P$ lines.

Proof. As in Theorem 11.1, for the simulator program of Listing A.2. The RAM memory representation is simpler since integer numbers are available. The representation of a RAM machine program and memory is as before, except that we assume input registers to be in a continuous range $0, \ldots, m$ and a constraint $\operatorname{maxm}(m)$ is added to the initial CHR goal. The auxiliary constraint $\operatorname{maxm}(m)$ indicates that the current highest initialized register address is $m$. When a register with a higher address $n$ is initialized, the auxiliary constraint initm/3 is used to initialize all addresses in the range $m+1, \ldots, n$. In addition to the $O(P)$ time to Introduce the encoded program and the $O(T)$ time to simulate it (again one CHR rule application per program instruction), the simulator needs $O(S)$ time to initialize memory.

Note that for a fixed RAM machine program which uses at least as much time as space, $P$ is a constant and $S$ is $O(T)$, so the CHR simulator RAMSIMUL takes $O(T)$ time and $O(S)$ space.

\subsection{Complexity meta-theorem}

The previous section dealt with the complexity properties of the theoretical CHR machine. In this section we investigate the complexity achievable in practice, i.e. on a RAM machine, which corresponds (more or less) to a real computer. As a result, we get a complexity meta-theorem; in Section 11.3 we will then apply this meta-theorem to the RAM machine simulator program RAMSIMUL. This will allow us to conclude that every algorithm can be implemented efficiently in CHR.

\subsubsection{CHR is Turing-equivalent}

In Chapter 4 we have discussed the compilation of CHR. Most of the CHR compilers translate CHR programs to host language programs. The resulting host language code can be executed on RAM machines by an interpreter or via further 
compilation steps. In this sense, we can say that CHR compilers convert CHR programs to (RAM machine) executable code. So, because clearly CHR compilers do exist, RAM machines can simulate CHR machines:

Lemma 11.3 The RAM machine can simulate CHR machines.

Because CHR machines can simulate Turing machines (Theorem 10.3), and Turing machines can simulate CHR machines (Lemma 2.9 and the above), we get the following rather unsurprising result:

Corollary 11.4 The CHR machine is Turing-equivalent.

\subsubsection{Complexity of the compiled code}

We now examine the practically achievable complexity of simulating a CHR machine on a RAM machine. We consider existing CHR compilers, in particular, the Leuven CHR system in hProlog (see Section 3.3.4).

First we recapture some compiler optimizations that are crucial for the time and space complexity of the generated code. We then define the dependency rank of a constraint occurrence, which will turn out to play a crucial role in the time complexity of executing a CHR machine.

\section{The refined operational semantics}

Most CHR implementations - in particular, the Leuven CHR system - follow the refined operational semantics $\omega_{r}$ of CHR. We have discussed the $\omega_{r}$ semantics in detail in Section 4.3.

Essential in the $\omega_{r}$ semantics is the notion of an active constraint. Query and body constraints are introduced from left to right. Once a constraint is introduced (or triggered by a Solve transition), it becomes active: its occurrences in the program are tried, in textual order. For every occurrence, the corresponding rule is tried by looking up matching partner constraints.

\section{Join ordering}

As discussed in Chapter 9, the order in which partner constraints are looked up is important. CHR compilers implement a strategy to pick the order of partner constraint lookups, called the join ordering. Given a CHR program $\mathcal{P}$, a join ordering strategy $\prec$ induces for every head constraint occurrence $c$ of $\mathcal{P}$, an order $\prec_{c}^{\mathcal{P}}$ on its partners. 


\section{Functional dependencies}

In Section 4.4.4 we already briefly discussed the notion of functional dependencies between constraint arguments (Duck and Schrijvers 2005). Informally, for a given CHR store, a constraint has a set semantics functional dependency on certain key arguments if there are no two instances of the constraint with the same key arguments.

In the refined operational semantics, set semantics functional dependencies can easily be enforced using simpagation rules. For example, to make sure that $c / 4$ has a functional dependency on the combination of its first and third argument, we add the following rule before all other rules:

$\mathrm{c}\left(\mathrm{A},,_{-}, \mathrm{B},_{-}\right) \backslash \mathrm{c}\left(\mathrm{A},_{-}, \mathrm{B},,_{-}\right) \Leftrightarrow$ true.

\section{Indexing}

As we have seen in Chapter 7, advanced data structures can be used to implement the CHR constraint store in an efficient way. The join ordering strategy determines which combinations of key arguments (look-up patterns) are used for partner constraint look-ups. The constraint store is implemented in such a way that for every combination of constraint arguments that is used as a look-up pattern, an index is maintained, for instance using hash-tables. As a result, all constraint store operations can be done in $O(1)$ amortized time. Furthermore, in terms of space, these data structures have an overhead of only a constant factor.

\section{Determined partners and dependency rank}

We now introduce the notions of determined partners and dependency rank. This will allow us to get a tighter upper bound on the complexity of finding suitable partner constraints.

Definition 11.5 (determined partner) Given a join ordering strategy $\prec, a$ $C H R$ program $\mathcal{P}$, and a set of valid goals $\mathcal{L G}$, we say an occurrence $c$ is determined by the $j$-th occurrence of constraint a iff for all execution states $\sigma$ that occur in a derivation $\left.d \in \Delta_{\omega_{r}}^{\mathcal{H}}\right|_{\mathbb{G}}$ for some valid goal $\mathbb{G} \in \mathcal{V} \mathcal{G}$, the following holds: if $\sigma$ is of the form $\langle[a \# i: j \mid \mathbb{A}], \mathbb{S}, \mathbb{B}, \mathbb{T}\rangle_{n}$ (that is, the occurrence subprocedure for the $j$-th occurrence of constraint $a$ is about to be executed), then a set semantic functional dependency for $c$ holds in state $\sigma$, where the key arguments of $c$ are fixed by $a$ and all partners $x$ for which $x \prec_{a}^{\mathcal{P}} c$.

In other words, a partner constraint $c$ is determined by a given (active) constraint occurrence of $a$ if the following holds: whenever the partner constraint $c$ is looked up in the corresponding occurrence subprocedure for $a$, there is at most one match for $c$ that needs to be considered. 
Definition 11.6 (dependency rank) The dependency rank of an (active) occurrence $a$ is the number of non-determined partner constraints of a.

The following example illustrates the above definitions. Consider the join ordering strategy $\prec$ used in the Leuven CHR system, the program RAMSIMUL, and the set of valid goals corresponding to the valid RAM machine instances.

Example 42 (dependency rank). Consider the fifth rule of RAMSIMUL:

$$
i(L, \operatorname{add}, B, A), m(B, Y) \backslash m(A, X), c(L) \Leftrightarrow m(A, X+Y), c(L+1) \text {. }
$$

For the active occurrence c(L), the following join ordering is used:

$$
\mathrm{c}(\mathrm{L}) \prec_{\mathrm{c}(L)}^{\text {RAMSIMUL }} i(\mathrm{~L}, \text { add }, \mathrm{B}, \mathrm{A}) \prec_{\mathrm{c}(L)}^{\text {RAMSIMUL }} \mathrm{m}(\mathrm{B}, \mathrm{Y}) \underset{\mathrm{c}(L)}{\text { RAMSIMUL }} \mathrm{m}(\mathrm{A}, \mathrm{X}) .
$$

Given the first argument $\mathrm{L}$ there can be only one matching i/4 constraint, which means the first argument of each $\mathrm{m} / 2$ constraint is known. Again, for a given first argument, there can be only one $\mathrm{m} / 2$. These functional dependencies are not enforced explicitly by simpagation rules; they are implied by the set of valid goals and the rules. Since all its partners are determined, the dependency rank of $c(L)$ is zero.

Similarly, the other occurrences of c/1 also have dependency rank zero. In general, the dependency rank is of course not always zero. Different occurrences in the same rule may even have a different dependency rank.

Example 43 (non-zero dependency ranks). Consider the following rule:

$$
m(A, B), m(B, C), m(D, E), m(E, F), m(G, H) \Leftrightarrow \ldots
$$

Assume that $\mathrm{m} / 2$ constraints are uniquely determined by their first argument (as in the RAMSIMUL program) and that the join ordering is the textual order. The dependency rank of $m(A, B)$ is two: $m(D, E)$ and $m(G, H)$ contribute to it while the other head constraints are determined partners. The dependency rank of $m(B, C)$ is three: $m(D, E), m(G, H)$, and $m(A, B)$ contribute to it.

(1) The notions of determined partners and dependency rank depend both on the power of the static analysis in the CHR compiler and on the dynamic program behavior. The former influences the quality of the join order strategy, while the latter determines the functional dependencies in derivations starting with valid goals. 


\section{Space reuse}

Every time a CHR constraint is removed, its representation in memory becomes garbage. If this garbage is not collected, we may get a space complexity which is not linear in the size of the constraint store. Using garbage collection we can get the right space complexity. However, in most Prolog systems, garbage collection has a time complexity linear in the number of live cells. This may result in a severe time complexity penalty.

In Chapter 8 we have tackled this problem by introducing memory reuse techniques called in-place updates and suspension reuse, inspired by compile-time garbage collection (Mazur 2004). These optimizations improve the space complexity by eliminating garbage, with only a small constant factor worst-case time overhead. The basic idea of suspension reuse is to store the representation of a removed constraint in a cache. Later, when a new constraint has to be inserted, a representation from the cache is used to build the new constraint representation. In-place updates are a special case where both the removal and the insertion are in the same rule, eliminating the need for an intermediate cache.

Lemma 11.7 Using suspension reuse with unlimited cache size, the following holds: If during a particular execution, the maximal number of constraints in the store is $S_{\max }$, then at any point in the execution, $S+C \leq S_{\max }$, where $S$ is the number of constraints in the store and $C$ is the number of elements in the cache.

Proof. Execution consists of a sequence of insertion and removal operations. We denote the store size after the $i$-th operation with $S_{i}$, the cache size with $C_{i}$, and their sum with $M_{i}=S_{i}+C_{i}$. Initially both the store and the cache are empty: $S_{0}=C_{0}=0$. Insertion increments the store size and decrements the cache size if it is not already empty (otherwise it simply remains empty). Removal decrements the store size and increments the cache size. We proceed by induction on the sequence length. For zero-length sequences the property holds trivially. Assuming the property holds for any sequence of length $n$, we show that it also holds for sequences of length $n+1$. Because of the induction hypothesis:

$$
\forall x \leq n: \quad M_{x} \leq \max _{i \leq n} S_{i} \leq \max _{i \leq n+1} S_{i}=S_{\max }
$$

We now only have to show that $M_{n+1} \leq S_{\max }$. If the last operation in the sequence is a removal, then $M_{n+1}=\left(S_{n}-1\right)+\left(C_{n}+1\right)=M_{n} \leq S_{\max }$. Assuming the last operation is an insertion, there are two cases: if $C_{n}>0$, then $M_{n+1}=$ $\left(S_{n}+1\right)+\left(C_{n}-1\right)=M_{n} \leq S_{\max }$; otherwise $C_{n}=0$ and $M_{n+1}=S_{n+1} \leq S_{\max }$. This concludes the proof.

With an unlimited cache size, no constraint representation ever becomes garbage (from the point of view of the underlying host language in which the CHR system is implemented): all constraint representations are alive, either because they are 
in the store or because they are in the cache. The above lemma shows that the right space complexity can be achieved for all CHR programs — without having to resort to run-time garbage collection in the host language.

\subsubsection{Complexity meta-theorem}

We now state the main result of this section:

Theorem 11.8 Given a CHR program $\mathcal{P}$ and a $\omega_{t}$ derivation d of length $T$ which has a corresponding $\omega_{r}$ derivation, for which the maximal store size is $S, m$ is the maximum dependency rank of the active occurrences in $\mathcal{P}$, and $p$ is the number of propagation rule applications in $d$; assuming the host language constraints used in the guards and bodies of the rules of $\mathcal{P}$ can be evaluated in constant time; the Leuven CHR system compiles $\mathcal{P}$ to hProlog code which has, for the given derivation $d$, a time complexity $O\left(T S^{m+1}\right)$ and a space complexity $O(S+p)$.

Proof. Assume the derivation $d$ consists of $s$ Solve steps, $i$ Introduce steps, and a Apply steps: $T=s+i+a$. The cost of finding a rule match for an active occurrence is $O\left(S^{m}\right)$ since this process basically boils down to nested iteration over the constraints in the store, where the nesting depth is the dependency rank. Indeed, determined partners only contribute a constant factor to this cost. Checking and extending the propagation history can be done in constant time if the history is implemented as a hash table. The Apply steps take $O(a)$ time plus the time to find rule matches. The latter has to be taken into account even if no rule matches are found and no rule is applied, so we attribute it to the Introduce and Solve steps. The Introduce steps take $O\left(i S^{m}\right)$ time: for every active occurrence of the introduced constraint, a matching rule is sought; for a fixed CHR program, the number of occurrences is bound by a constant. Every Solve step potentially triggers all the $O(S)$ constraints in the store, so the Solve steps may take up to $O\left(s S^{m+1}\right)$ time. Since $s, i$, and $a$ are all $O(T)$, the total time complexity is $O\left(T S^{m+1}\right)$. The $O(S+p)$ space complexity can be achieved using suspension reuse with unlimited cache size, as shown in Lemma 11.7.

Theorem 11.9 If in the previous theorem, the CHR program is ground (i.e. all constraint arguments are ground), then $O\left(T S^{m}\right)$ time complexity can be achieved.

Proof. See the previous proof. Constraints are never triggered in ground programs. This reduces the complexity of one Solve step to a constant.

Formulating the above result in terms of CHR machines we get:

Corollary 11.10 A ground CHR machine without propagation rules, with time complexity $T$ and space complexity $S$, can be simulated on a RAM machine with 
time complexity $O\left(T S^{m}\right)$ and space complexity $O(S)$, where $m$ is the maximum dependency rank of the active occurrences in the program of the CHR machine.

\subsection{Complexity-wise completeness}

Now we use the general result of Corollary 11.10 to analyze the time and space complexity of the RAM simulation (compiler-generated code) of the CHR machine $\mathcal{M}_{\mathrm{RAM}}$, which itself simulates RAM machines.

Theorem 11.11 The $C H R$ machine $\mathcal{M}_{\mathrm{RAM}}$ with program RAMSIMUL, with time complexity $T$ and space complexity $S$ can be simulated on a $R A M$ machine with time complexity $O(T)$ and space complexity $O(S)$.

Proof. It can be easily verified that the dependency rank for all occurrences of c/1 is zero given the join ordering strategy used in the Leuven CHR system (cf. Example 42). For the other occurrences this is slightly less straightforward. Valid goals have exactly one $\mathrm{c} / 1$ constraint. All rules remove one $\mathrm{c} / 1$ constraint and optionally insert another one in the body (indirectly in the case of the rule for the init instruction). Hence there is never more than one $c / 1$ constraint - in other words, c/1 has a set semantics functional dependency on the empty key. If the join order strategy does the lookup of $\mathrm{c} / 1$ first, the remaining partners become determined.

Since the dependency rank is zero for all occurrences, applying Corollary 11.10 (with $m=0$ ) results in the desired complexities.

(1) In practice we will add a rule like "c(_) $\Leftrightarrow$ fail" at the very end of the RAMSIMUL program. In the refined semantics, for valid goals, this rule is never applied. However, it allows the never stored optimization (see Section 4.4 .3 on page 72 ) to deduce that all partners of $c / 1$ are passive. This implies that only the dependency ranks of the occurrences of $c / 1$ have to be considered, simplifying the above proof.

We conclude that "everything can be done efficiently in CHR":

Corollary 11.12 For every (RAM machine) algorithm which uses at least as much time as it uses space, a CHR program exists which can be executed in the Leuven CHR system in hProlog, with time and space complexity within a constant from the original complexities.

Proof. Consider any algorithm which can be expressed as a RAM machine program with a program of $P$ lines, and let its time and space complexities be $T$ and $S$, respectively. Because of Theorem 11.2, a CHR(Prolog) machine $\mathcal{M}_{\mathrm{RAM}}$ with program RAMSIMUL exists, which simulates that RAM machine in $O(S)$ space and $O(T)$ time, since $P$ is a constant and $S$ is $O(T)$. Now, because of the 
above Theorem 11.11, executing RAMSIMUL in the Leuven CHR system also takes $O(T)$ time and $O(S)$ space.

One may expect to pay some performance penalty for using a very high-level language like CHR, so it is comforting that at least we can always get the asymptotic complexities right.

\subsubsection{Discussion}

We have investigated the complexity of Constraint Handling Rules by introducing the CHR machine, a model of computation based on the operational semantics of CHR. Besides the expected result that CHR is a Turing-equivalent language, we have demonstrated the much stronger result that every RAM machine program can be implemented as a CHR program which has the same asymptotic time and space complexities if executed in the Leuven CHR system. In other words, the current state-of-the-art in CHR compilation allows CHR programmers to implement any algorithm with the best possible complexity. As far as we know, CHR is the first declarative language for which such a complexity-wise completeness result has been proven to hold within the pure part of the language, i.e. without imperative extensions to the language - see also Section 11.4.

Figure 11.1 summarizes the results. It gives an overview of the relationships between three models of computation: Turing machines, RAM machines, and CHR machines. An arrow between $A$ and $B$ indicates that $A$ can be simulated on $B$. Labels indicate the relevant section or theorem and the time complexity of simulating a $T$-time, $S$-space $A$ on $B$. Note that the $\tilde{O}\left(\left(T S^{m+1}\right)^{4}\right)$ bound for simulating a CHR machine on a Turing machine can be obtained by simulating the CHR machine on a RAM machine, and then simulating that RAM machine on a Turing machine.

\subsubsection{Register initialization}

Our definition of the space complexity of a RAM machine (see Definition 2.8 on page 23) is based on that of Savitch (1978). It counts all registers in the used address range, whether or not each individual register was effectively used. In the literature, other definitions of RAM machine space complexity only take the used registers into account (van Emde Boas 1990). If we would use such a definition, the above results no longer hold in general: for a program that uses the registers in a sparse way, the RAM machine simulator RAMSIMUL of Listing A.2 would use more space (and thus possibly also more time) than the original RAM machine. The reason is that the simulator initializes the entire register range.

However, by relying on the refined operational semantics, we can implement the init instruction in a different way — see Listing 11.1. The auxiliary constraints $\operatorname{maxm} / 1$ and initm/3 are not needed in this version of the simulator. Of course, 


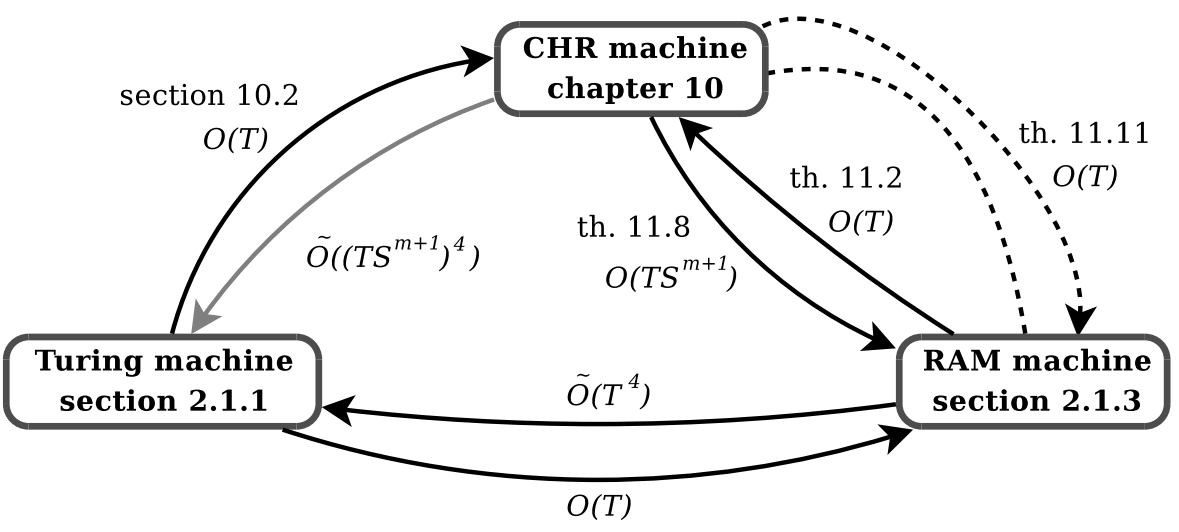

Figure 11.1: Relationships between Turing, RAM, and CHR machines

Listing 11.1: Alternative register initialization, using the $\omega_{r}$ semantics

$\operatorname{prog}(L$, init, $A), m(A, B), m(B,-) \backslash p c(L) \Leftrightarrow p c(L+1)$.

$\operatorname{prog}(L$, init, $A), m(A, B) \quad \backslash p c(L) \Leftrightarrow m(B, 0), p c(L+1)$.

this alternative program is no longer confluent; its correctness depends on the order of rule applications enforced by the refined semantics. Indeed, under the $\omega_{r}$ semantics the second rule is applied only if the first rule cannot be applied because of the absence of a corresponding $\mathrm{m} / 2$ constraint. Checking for absence of constraints cannot be done in a confluent way — see also the work on extending CHR with negation-as-absence (cf. Section 3.4.2).

(n) In Section 11.4 we will assume, for simplicity, that registers are initialized exactly once before use. In that case, the second rule of Listing 11.1 suffices and we get a confluent program. However, in general, it is not possible, without complexity penalty, to rewrite every RAM machine program in such a way that it initalizes all used registers exactly once.

\subsubsection{Related and future work}

We have already mentioned the complexity meta-theorem by Frühwirth (2002a, $2002 \mathrm{~b}$ ) in Section 3.2.3. Since it assumes a very naive implementation of CHR, the resulting bounds are rather crude. For the RAM machine simulator program RAMSIMUL simulating a $T$-time RAM machine, the upper bound predicted by Frühwirth (2002a) is $O\left(T^{6}\right)$ - quite far from the $O(T)$ bound of Lemma 11.11. 
We have explicitly decoupled the two steps in the approach of Frühwirth by introducing CHR machines. If suitable termination orders can be found, they can be used to show an upper bound on the complexity of the CHR machine. This is the first step. However for programs that are non-terminating in general, like the RAM simulator, or for which no ranking can be found, other techniques have to be used to prove complexity properties. For example, we have shown that the steps of the CHR machine $\mathcal{M}_{\mathrm{RAM}}$ correspond to the steps of the RAM machine it simulates, terminating or not. The second step corresponds to the question of how efficiently a CHR machine can be executed in practice. Recent work on optimizing compilation of CHR has allowed us to achieve much tighter bounds.

Future work. It is an open problem whether a result similar to the the linear speedup theorem (Hartmanis and Stearns 1965) can be demonstrated for CHR machines. To improve the time complexity of a CHR machine, one could try to reduce the number of Apply steps by combining rules, and the number of Introduce steps by combining constraints. It is not clear whether such a reduction is possible in general. This is somewhat related to partial evaluation techniques.

Although we are convinced that every algorithm can be implemented with an elegant CHR program, it remains a useful research topic to construct good CHR implementations of existing (or new!) algorithms (cf. Chapter 5).

The RAM machine simulator is a ground CHR program without propagation rules. In a sense, our result implies that non-ground constraints (which may be triggered) and propagation rules (that require checking and maintaining a propagation history) are not strictly needed for complexity-wise completeness. However, since non-ground constraints and propagation rules are widely used (especially in the traditional constraint solver programs), improving the complexity of their implementation is still very useful.

\subsection{Other declarative languages}

Many Turing-complete programming languages have the complexity-wise completeness property we have shown for $X=\mathrm{CHR}(\mathrm{hProlog})$ : "every algorithm can be implemented in language $X$ with the right time and space complexity". For instance, in all imperative languages that we are aware of, it is a straightforward exercise to construct a RAM machine simulator and show that it has the right complexity. After all, the basic ingredients needed for a RAM machine simulator are directly available in most imperative languages.

However, for higher level, declarative languages, the complexity-wise completeness property is far less trivial. The time and space complexity of a program depends more crucially on optimizing compilation.

In this section we briefly investigate whether some other declarative languages allow an efficient implementation of a RAM machine simulator, given the current 
state of the art. To keep this section concise, we will only consider one well-known (and supposedly representative) language for each of these declarative paradigms: logic programming (Prolog), functional programming (Haskell), term-rewriting (Maude), and rule-based programming (Jess).

- Whether or not the complexity-wise completeness result holds for some language clearly depends largely on the properties of its compiler. A pathological compiler can be conceived that detects the pattern of a 'RAM machine simulator program' as a special case and produces special, hardwired output with the desired complexity properties. Such 'optimizations' have to be ruled out since they are not applicable to a large class of programs. However, where exactly to draw the line between 'cheating' and 'benign' optimizations is not straightforward. In this section we only consider the 'pure' fragments of the different declarative languages. Some of the languages also have imperative extensions (e.g. setarg/3 in Prolog) but we consider those a form of 'cheating' - for the CHR(Prolog) complexity-wise completeness result we did not need any extensions of CHR, and the compiler optimizations we needed are 'benign' in the sense that they are applicable to a large class of CHR programs.

\subsubsection{Sufficient ingredients}

By closely investigating the definition of RAM machines (see Section 2.1.3), we can identify the crucial programming language features needed to obtain a complexitywise completeness result in the same way as in the previous section, that is, by constructing a RAM machine simulator. An efficient RAM machine simulator can be implemented if the following ingredients can be implemented:

1. A mechanism for iteration, such that iterating $n$ times takes $O\left(n T_{s}\right)$ time and $O\left(S_{s}\right)$ space if evaluating the stop condition takes $T_{s}$ time and $S_{s}$ space;

2. The arithmetic operations, with the same complexity as the corresponding RAM machine arithmetic operations;

3. An if-then-else language construct and evaluation of (syntactic) equality and inequality conditions, both in constant time and zero space;

4. Dynamically growing arrays which allow $n$ insertion, $g$ lookup, and $s$ update operations in $O(n+g+s)$ time and $O(n)$ space.

Most declarative languages do not offer iteration (the first ingredient) as a basic language construct, but many implementations convert tail recursion to iteration, so the first ingredient will not be the problem. The second and third ingredient are directly and/or implicitly available in all the languages we consider. 


\subsubsection{Arrays in declarative languages}

The remaining ingredient, an efficient dynamically growing array, is the one that seems to be the most difficult to implement. In this section we try to implement, as efficiently as possible, the functionality of arrays in several declarative languages, and we construct corresponding RAM machine simulator programs. In the next section we will experimentally compare the performance of the resulting programs.

\section{Logic programming languages}

We use the term pure Prolog to denote the Prolog language as described by Clocksin and Mellish (1984), without the assert and retract built-ins. Clearly, if non-pure Prolog extensions - for example global variables, mutable terms, and assert/retract - are allowed, there is an efficient RAM machine simulator implementation: consider, for instance, the code the $\mathrm{CHR}$ (hProlog) compiler generates for the RAMSIMUL program.

To the extent of our knowledge, there is no Prolog system which allows an efficient pure Prolog implementation of dynamically growing arrays. Association lists, available in many Prolog systems as a standard module called assoc, can be used instead. The implementation of assoc that is used in hProlog is based on an implementation by Mats Carlsson (which was based on an implementation by Richard O'Keefe) based on AVL-trees (Adelson-Velsky and Landis 1962). Lookup, insertion and update take $O(\log n)$ worst-case time. Listing A.3 gives a Prolog program which uses association lists to implement a RAM simulator. Association lists are used to represent both the RAM machine program and the memory cells.

Mercury. Mercury (Somogyi, Henderson, and Conway 1996) is a strongly-typed, high-performance logic programming language. The Mercury system includes an array module. However, the procedures of this module are not written in the Mercury language itself, but directly in the target languages ( $\mathrm{C}, \mathrm{C \#}$, and Java).

In an experimental development branch of Mercury, compile-time garbage collection (CTGC) has been added by Mazur (2004) — see also Chapter 8. This allows automatic structure reuse in a large class of Mercury programs. Perhaps CTGC allows a reasonably efficient pure Mercury implementation of growing arrays: using AVL-trees and with in-place updates thanks to CTGC, it should be possible to perform $n$ insertions, $g$ lookups, and $s$ updates in $O((n+g+s) \log n)$ time and $O(n)$ space. We have not been able to test Mercury programs experimentally since CTGC is not yet available in the main release of the Mercury system. 


\section{Functional programming languages}

Haskell (Hudak, Hughes, Jones, and Wadler 2007) is a modern typed, lazy, purely functional language. Most Haskell systems include the Data. Array module in their standard libraries. This module efficiently implements arrays, but it is not implemented in Haskell itself. The fastest pure Haskell implementation of arrays we could find is available in the standard Data. IntMap module, which is based on an implementation of Patricia trees (Morrison 1968) by Okasaki and Gill (1998). This data structure allows memory cell look-ups, updates, and insertion (initialization) in $O(\min (n, W))$ time, where $W$ is the number of bits in an Int. On our test platform, $W=32$ so the operations can be considered to be constant-time. However, since the updates are not done in-place, the space complexity is $O(n+s)$ instead of $O(n)$. Listing A.4 gives the Haskell program we have tested. We have tested both a "lazy" version (see Listing A.4) and a "strict" version. The latter naively forces all lazy thunks immediately to weak head normal form (WHNF); it differs from the lazy version on two accounts only. Firstly, the fields of the Instr datatype are declared to be strict. Secondly, each function application $f \mathrm{e}$ is transformed into let $\mathrm{x}=\mathrm{e}$ in $\mathrm{x}$ ' $\mathrm{seq}^{\text {' }} \mathrm{f} \mathrm{x}$, which forces the subexpression $\mathrm{e}$ to WHNF before evaluating the main expression $f$ e.

\section{Term-rewrite systems}

Maude $^{1}$ (Clavel, Durán, Eker, Lincoln, et al. 2002) is a system for declarative programming in rewriting logic. It features efficient rewriting of terms with associativecommutative (AC) operators using the stripper-collector matching algorithm of Eker (2003). Listing A.5 gives the Maude program we have tested. This program is directly derived from the CHR rules. As in CHR, the collection data structures and the operations on them are implicit. In this sense CHR and Maude are higher level languages than Prolog and Haskell.

Unfortunately, for the rules of Listing A.5, the current implementation of Maude is not able to use its most efficient matching algorithm. By making the data structure operations more explicit (using the Map\{Int, Int $\}$ module) we obtain a more efficient program in which memory cell lookups take only logarithmic time. It is given in Listing A.6.

\section{Rule engines}

Jess $^{2}$ (Friedman-Hill 2003) is considered to be one of the fastest rule engines. Like its ancestor CLIPS (Giarratano and Riley 1994), it uses the RETE algorithm of Forgy (1982). Listing A.7 gives the Jess program we have tested.

\footnotetext{
${ }^{1}$ Maude home page: http://maude.cs.uiuc.edu/

${ }^{2}$ Jess home page: http://www.jessrules.com/
} 
In a sense, Jess is higher level than Prolog and Haskell because the data structures are implicit. It is lower level than CHR and Maude (as in the Maude program of Listing A.5) because the data structure operations (assert, retract, and modify) are explicit. Moreover, as far as we know, Jess does not have the join ordering optimization. We have picked the best possible order of rule heads in the simulator program of Listing A.7. If the heads are written in a different order, performance suffers. This is another sense in which Jess can be considered to be a lower level language than CHR - thanks to automatic join ordering, CHR programmers do not have to worry about the order of the head constraints.

(0) In general, the eager matching RETE algorithm is asymptotically slower than the lazy matching LEAPS algorithm (Miranker, Brant, Lofaso, and Gadbois 1990) on which the CHR execution mechanism (under the refined operational semantics) is based. In the specific case of the RAM machine simulator program of Listing A.7, this disadvantage of RETE does not emerge.

\subsubsection{Experimental results}

To test the performance of the RAM machine simulator, we have executed the simulator for three benchmark RAM machine programs (see Table B.4). The results are shown in Table B.5, which lists the execution times of running the RAM simulator benchmarks of Table B.4 in different RAM simulator implementations.

Benchmark 1: "Loop". The first benchmark, "Loop", performs $O(n)$ operations and uses only three memory cells. The space usage is constant in CHR, Prolog, Maude, and strict Haskell, but not in lazy Haskell: in this example, lazy evaluation creates $O(n)$ lazy thunks so it needs $O(n)$ space. The time complexity is linear in all systems. The Jess program is the slowest: it takes more than twice the time of the naive Maude program. The naive Maude program is roughly three times slower than the one that uses Map, which is about as fast as CHR. The CHR program with type detail ${ }^{3}$ level 2 is almost four times faster than the CHR programs with type detail level 1 or 0 , about twice as slow as the Prolog version and four times slower than strict Haskell.

Benchmark 2: "MFib". In the second benchmark, "MFib", $O(n)$ memory cells are used. Naive Maude and CHR without type information do not get the time complexity right: the $O(n)$ lookups seem to take $\tilde{O}\left(n^{2}\right)$ time. Prolog, Haskell, and Maude with Map get the time complexity almost right: $\tilde{O}(n)$ instead of $O(n)$. Both Prolog and Haskell use too much space. In the case of Prolog and strict

\footnotetext{
${ }^{3}$ See Table 7.1 on page 126 . Type detail level 2 corresponds to types and modes (crucially, +dense_int for the first argument of $\mathrm{m} / 2$ ); detail level 1 corresponds to modes only (all ground); detail level 0 corresponds to no argument information.
} 


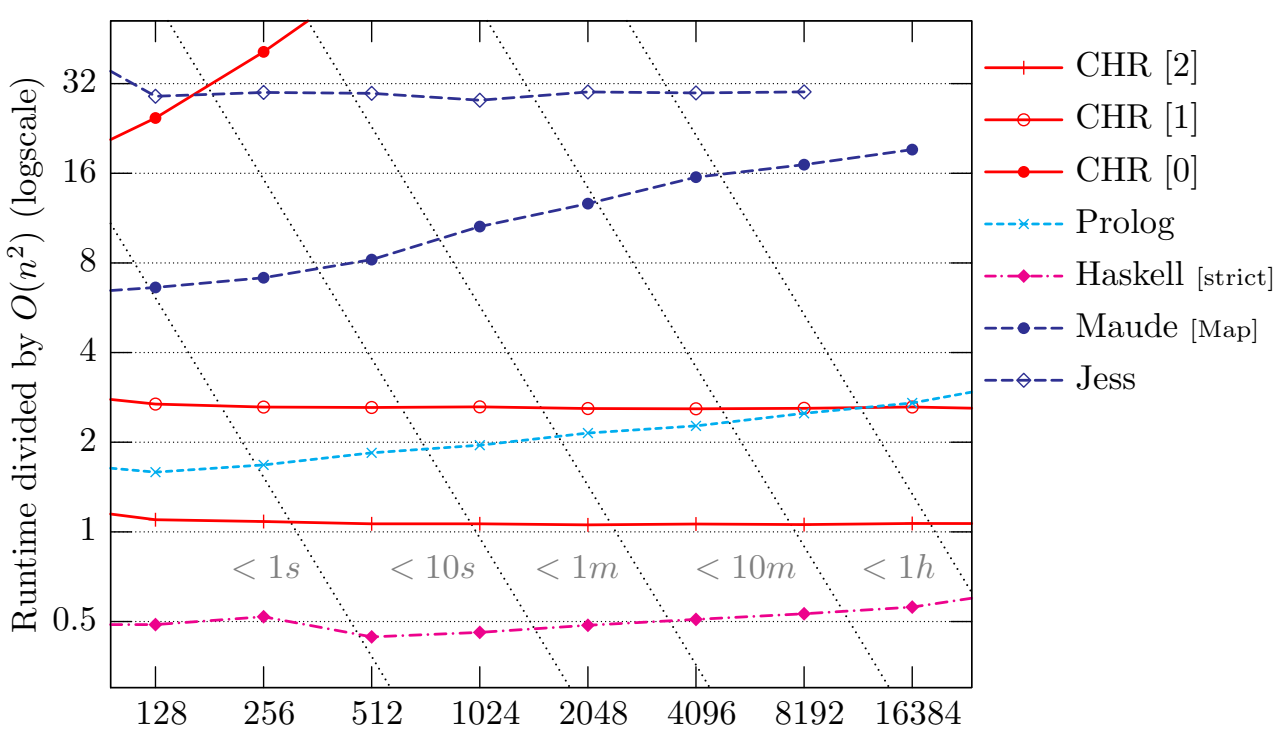

Figure 11.2: Results of the "NLoop" benchmark (cf. Table B.5)

Haskell, garbage collection takes an increasingly higher proportion of the runtime. In lazy Haskell, even garbage collection does not prevent running out of memory. CHR with type detail level 1 and Jess get the right time and space complexity; Jess is more than ten times slower and also uses significantly more space.

Benchmark 3: "NLoop". The last benchmark, "NLoop", performs $O\left(n^{2}\right)$ updates on $O(n)$ memory cells. As expected from the results of the previous benchmark, only CHR with type detail level $>0$ and Jess get the $O\left(n^{2}\right)$ time complexity completely right. CHR is between 10 and 30 times faster than Jess. Prolog, (strict) Haskell and Maude with Map achieve $\tilde{O}\left(n^{2}\right)$ time complexity.

To aid us in interpreting the benchmark results, we have plotted the runtimes divided by the expected complexity $O\left(n^{2}\right)$ in Figure 11.2. If the resulting curve is horizontal, the expected complexity is achieved. Both in constant factors and unwanted non-constant factors, the strict Haskell version is better than the Prolog version, which is in turn better than the Maude version.

\subsubsection{Summary}

We can classify the languages as shown in Table 11.1:

- Jess and CHR (with modes) achieve optimal time and space complexity; 


\begin{tabular}{|c|c|c|c|}
\hline Language & Time & Space & Complexity-wise complete? \\
\hline CHR [0] & not optimal & optimal & space-only \\
CHR [1] & optimal & optimal & yes \\
CHR [2] & optimal & optimal & yes \\
Prolog & almost optimal & not optimal & almost (time-only) \\
Haskell (strict) & almost optimal & not optimal & almost (time-only) \\
Haskell (lazy) & not optimal & not optimal & no \\
Maude (naive) & not optimal & optimal & space-only \\
Maude (Map) & almost optimal & optimal & almost \\
Jess & optimal & optimal & yes \\
\hline
\end{tabular}

Table 11.1: Language classification in terms of complexity-wise completeness

- Maude with Map has optimal space complexity (as far as we can tell) and gets within a (large) polylogarithmic factor from optimal time complexity;

- CHR without type declarations and naive Maude have optimal space complexity, but they do not achieve optimal time complexity;

- Prolog and strict Haskell have a time complexity which is within a polylogarithmic factor from optimal, but their space complexity is not optimal;

- lazy Haskell does not get close to optimal space complexity, and this ruins its time complexity.

For the declarative programming languages we have tested in this section, the apparent inability to obtain optimal complexity in the pure language is not really a problem in practice. If needed, programmers can use non-pure language elements like the setarg/3 built-in in Prolog and the array modules in Mercury and Haskell. It is not clear to us whether and how pure logic or functional programming languages can be implemented in a way that allows an efficient implementation of a RAM simulator.

\subsection{Constant factors}

We have shown the complexity-wise completeness of CHR - every algorithm can be implemented in CHR with the right asymptotic time and space complexity. However, the constant factors hidden behind the notion of asymptotic complexities could be huge. In fact, they could be so huge as to be completely paralyzing in practice. In this section we investigate these constants experimentally. 


\begin{tabular}{rl|l} 
movl & $\$ 1, \%$ eax & $\mathrm{m}(1,1)$, \\
movl & $\$ 1000000, \%$ ecx & $\mathrm{m}(2,1000000)$, \\
movl & $\$ 0, \%$ edx & $\mathrm{m}(3,0)$, \\
.L1: & $\mathrm{iddl}(1, \mathrm{add}, 1,3)$, \\
subl & $\%$ eax, \%edx & $\mathrm{i}(2, \mathrm{sub}, 1,2)$, \\
je & .L5 & $\mathrm{i}(3, \mathrm{cjmp}, 2,5)$, \\
jmp &. L1 & $\mathrm{i}(4, \mathrm{mp}, 1)$, \\
.L5: & & $\mathrm{i}(5$, halt $)$, \\
& & $\mathrm{c}(1)$.
\end{tabular}

Figure 11.3: Assembler code and corresponding RAM machine query

\subsubsection{Complexity-wise completeness in practice}

In principle, every algorithm can be implemented in CHR using the RAM simulator program. Of course, this does not result in a natural and elegant CHR program, but at least the resulting CHR program has the right time and space complexity.

Consider the following very simple $\mathrm{C}$ program:

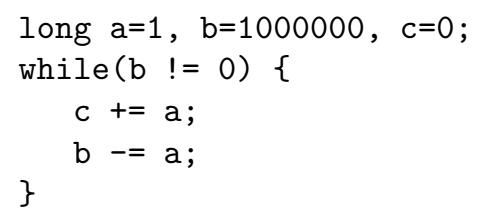

This C program corresponds to the Intel assembler code shown in Figure 11.3 (on the left hand side). The assembler code can also be seen as a query for the RAM machine program RAMSIMUL, as shown in the right hand side of Figure 11.3. The observant reader will notice that this is in fact just the "Loop" benchmark that was discussed in the previous section.

By translating assembler code to a RAM simulator query, we get a CHR program with the same asymptotic time and space complexity: in this example, both the CHR program and the assembler code take linear time and constant space.

Although the CHR(Prolog) RAM simulator executes such RAM programs with the correct asymptotic complexity, the execution time is about ten thousand times larger than that of the original assembler code program for this example: the RAM simulator takes about 10 seconds while the assembler program runs in 1.6 milliseconds. In other words, the computational power of a Pentium 4 is reduced to that of a Commodore 64 .

Of course no sane programmer would write CHR programs in this way not just because of the debilitating slowdown: such programs also lack desirable properties of CHR programs (conciseness, readability, adaptability, incrementality, concurrency, ...) that are often obtained naturally in hand-written CHR programs. Hence it remains necessary to manually construct CHR programs. 


\subsubsection{Experimental evaluation}

Chapter 5 discussed several CHR programs that implement classical algorithms. In this section we investigate the performance of two of these hand-written CHR programs. We compare their performance to that of an efficient reference implementation, in the low-level language $\mathrm{C}$, of the same algorithms. The goal is to obtain an estimate of the (constant factor) performance penalty for using CHR.

As we have seen in Chapter 7, the Leuven CHR system allows the programmer to specify optional type and mode declarations for the constraint arguments (see also Table 7.1 on page 126). The information is used for optimizing compilation. The original CHR system in SICStus Prolog, by Holzbaur and Frühwirth (1998), does not have a mechanism to provide such declarations. In the Java CHR system, precise type declarations are obligatory since Java is a typed language.

\section{Union-find}

Consider again the union-find program of Section 5.1 (Listing 5.2 on page 77 ). Table B.6 lists the execution times for this CHR program in the different CHR systems. We compare these results against a very efficient $\mathrm{C}$ implementation ${ }^{4}$. In order to achieve the optimal complexity, type detail level 1 is needed (see Table 7.1). With type detail level 2, the high-level CHR(hProlog) implementation is roughly 10 times slower than the direct low-level implementation in C. The results are plotted in Figure 11.4. The benchmark consists of performing $n$ make operations, followed by $n$ random union operations and $n$ random find operations.

To get an idea of the constant factors involved in space usage, consider the following numbers. The $\mathrm{C}$ program uses only one array to represent the entire data structure; every element takes one word (4 bytes): positive integers represent the index of the parent, negative integers represent the rank of a root. In contrast, the $\mathrm{CHR}$ (hProlog) program uses 9 words to represent an element: two arrays are used (one for roots, one for non-roots), which contain pointers to 7-word suspension terms: one word for the wrapper functor, two for the constraint arguments, one for the identifier, three for the state (see also Section 4.2.1). Hence for the union-find algorithm, the $\mathrm{CHR}$ version uses about ten times as much space as the $\mathrm{C}$ version.

\section{Dijkstra's algorithm with Fibonacci heaps}

In Section 5.2 we have discussed a CHR implementation of the shortest path algorithm of Dijkstra (1959), which uses the Fibonnacci heaps data structure of Fredman and Tarjan (1987). We have compared the performance of the DIJKSTRA program (see Listing 5.3 on page 79 ) to that of a $\mathrm{C}$ implementation ${ }^{5}$ by Cherkassky et al. (1996). The results are listed in Table B.7 and plotted in Figure 11.5.

\footnotetext{
${ }^{4}$ Written by Ariel Faigon, based on a version by Robert Sedgewick. The source code is available at http://www. yendor.com/programming/minauto/ufind.c

${ }^{5}$ The source code is available at: http://www.avglab.com/andrew/soft.html
} 


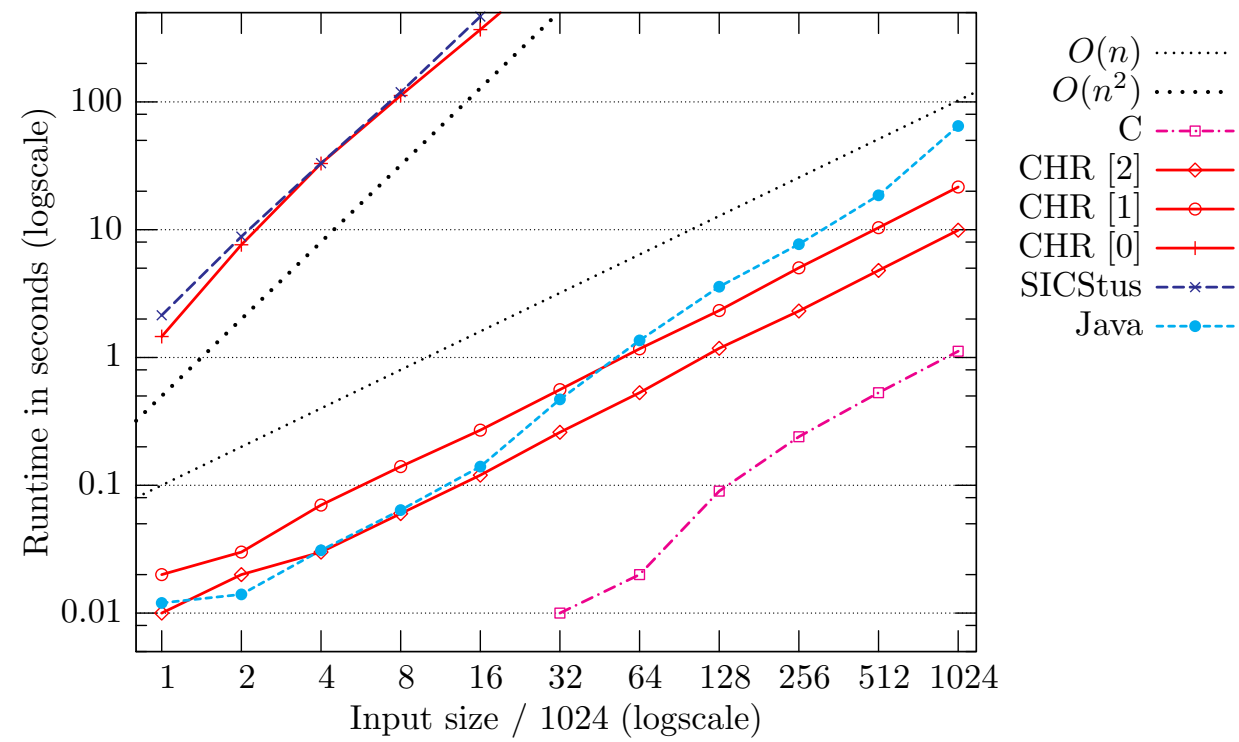

Figure 11.4: Results of the "Union-find" benchmark (cf. Table B.6)

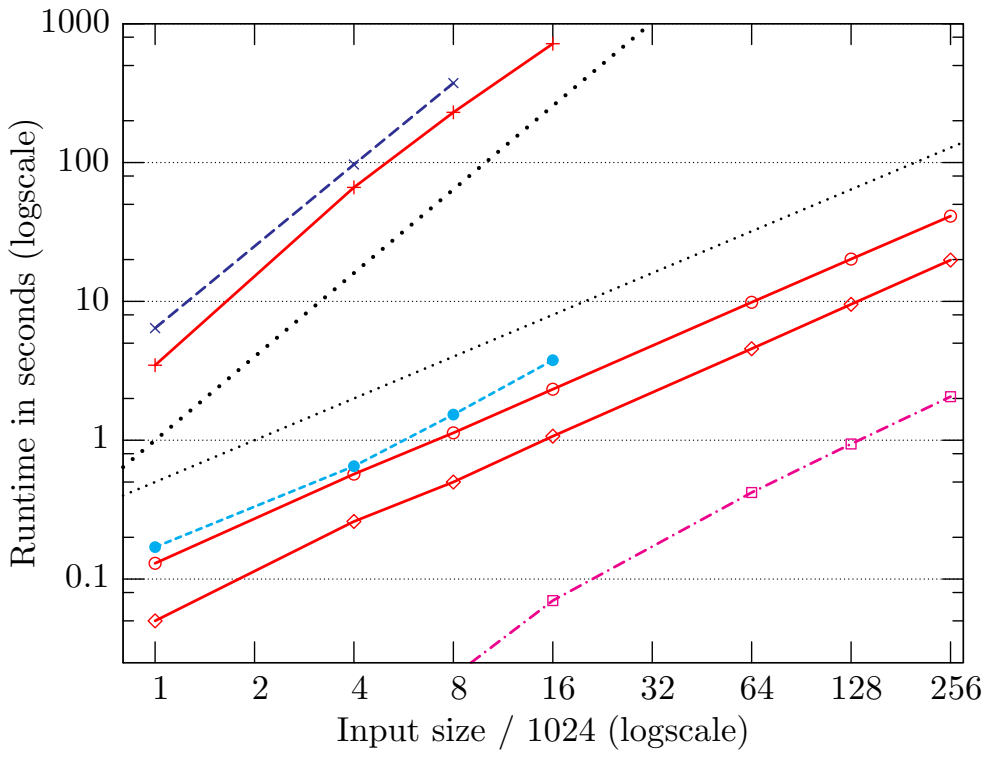

$$
\begin{aligned}
& O(n) \\
& O\left(n^{2}\right) \cdots . . \\
& \text { C -.- }- \text {-. } \\
& \text { CHR [2] } \smile \\
& \text { CHR [1] } \longrightarrow \\
& \text { CHR [0] — } \\
& \text { SICStus --*-- }
\end{aligned}
$$

Figure 11.5: Results of the "Dijkstra" benchmark (cf. Table B.7) 
Note the overall resemblance between Figure 11.5 and Figure 11.4. Again, type detail level 1 (see Table 7.1) is needed to achieve optimal complexity. Without any information, the program exhibits a quadratic time complexity, because the default data structure does not allow constant time look-ups of ground constraints. When groundness information is available, the optimal $O(n \log n)$ time complexity is achieved. The time gap between the hProlog CHR program (type detail level 2) and the $\mathrm{C}$ program is - again - a constant factor of about 10 .

The difference in space usage is less pronounced than for the union-find algorithm. The CHR program uses about three times more space than the $\mathrm{C}$ program: for an input size of $256 \mathrm{k}$ nodes, the $\mathrm{C}$ program uses 23 megabytes, while the CHR(hProlog) program needs 63 megabytes.

\subsubsection{Conclusion: $\mathrm{CHR}(\mathrm{hProlog}) / \mathrm{C} \approx 10$}

The above results indicate that the constant time factor separating $\mathrm{CHR}(\mathrm{hProlog})$ from $\mathrm{C}$ is approximately 10 . In terms of space usage, the constraint representation has a fixed overhead: in programs using very light-weight data representations (e.g. union-find), this results in a relatively large constant space factor (e.g. 10); in programs with more complicated representations (e.g. Fibonacci heaps), the constant space factor is smaller (e.g. 3). Future and ongoing work in CHR compiler optimization will further reduce these factors. In particular, the space overhead can be much reduced by further specializing the constraint representation.

Extrapolating from the above examples, combined with the results of the previous section, we can summarize our results as follows: "The current state of the art in CHR and Prolog systems suffices to implement any algorithm in CHR(Prolog), in a natural and high-level way, with a time and space complexity which is within a constant factor of 10 from the best-known implementation in any other programming language." Clearly, the notion of "natural and high-level" is rather vague and open for interpretation, so unlike the complexity-wise completeness result of Corollary 11.12 , the above statement cannot be proved mathematically.

Future work. More empirical evidence can be gathered by implementing more algorithms in CHR and comparing the performance with that of implementations in low-level programming languages. One could for instance attempt to implement, in CHR, a (large) subset of the algorithms described in classic books on algorithms, for example (Knuth 1998). Perhaps this approach allows to make conclusions about the kind of algorithms for which CHR is more or less suitable.

A more direct approach would be to construct a direct translation of some (subset) of an imperative language (e.g. some subset of Java) to CHR, and measure the constant factor gap - knowing that this gap will be larger for a general translation scheme than for hand-tailored 'free translations'. 


\title{
Chapter 12
}

\section{Generalized CHR Machines}

\author{
"Beware of bugs in the above code; I have only proved it correct, \\ not tried it."
}

- Donald Knuth (1938-)

In Chapter 10 we have introduced CHR machines that are based on the abstract operational semantics $\omega_{t}$. This original definition of CHR machines is rather restricted: it requires a CHR program that is $\Delta_{\omega_{t}}^{\mathcal{H}}$-deterministic.

This chapter deals with various generalizations of the CHR machine. First, in Section 12.1, we introduce the notion of strategy classes and generalized confluence. Then, in Section 12.2, we introduce generalized CHR machines, that follow an arbitrary strategy class (not necessarily the strategy class corresponding to the abstract semantics) and whose programs don't need to be deterministic. In Section 12.3 we introduce non-deterministic CHR machines (NCHR machines), and in Section 12.4 we introduce self-modifying CHR machines, also known as CHR machines with a stored program (CHRSP machines). We conclude in Section 12.5.

\subsection{Strategy classes}

The $\omega_{t}$ operational semantics is very nondeterministic, in the sense that for most programs, the number of possible derivations is very large. This is of course the reason why the confluence property is crucial for program correctness.

However, all CHR implementations restrict the nondeterminism of the $\omega_{t}$ semantics. While still guaranteeing rule application until exhaustion, they usually impose some order in which rules are tried. In effect, they instantiate the $\omega_{t}$ semantics; examples are the refined operational semantics $\omega_{r}$ (cf. Section 4.3) and the priority semantics $\omega_{p}$ (cf. Section 4.1.4). 
In this section we examine instantiations of the $\omega_{t}$ semantics in a general formal framework. We introduce the notion of an execution strategy which fixes the derivation for all initial goals. A set of execution strategies is called a strategy class. We then weaken the notion of confluence to that of generalized confluence w.r.t. some strategy class.

\subsubsection{Execution strategies}

Definition 12.1 (execution strategy) An execution strategy fixes the derivation for every initial state. Formally, $\xi$ is an execution strategy for a program $\mathcal{P}$ if $\xi \subseteq \Delta_{\omega_{t}}^{\mathcal{H}}(\mathcal{P})$ and for all goals $\mathbb{G} \in \mathcal{G}_{\mathcal{P}}^{\mathcal{H}}$, we have that $\left.\xi\right|_{\mathbb{G}}$ is a singleton.

The set of all execution strategies for a program $\mathcal{P}$ is denoted by $\Omega_{t}^{\mathcal{H}}(\mathcal{P})$. Clearly not every execution strategy can be implemented:

Example 44 (non-computable execution strategy). With the right execution strategy, the following program solves the halting problem:

$$
\begin{aligned}
& \text { check_halts }(\mathrm{TM}) \Leftrightarrow \text { true. } \\
& \text { check_halts }(\mathrm{TM}) \Leftrightarrow=>\text { fail. }
\end{aligned}
$$

We can imagine an execution strategy that applies the first rule if the Turing machine encoding TM in the input query check_halts (TM) halts, and otherwise applies the second rule.

As the above example illustrates, we need a more realistic notion of execution strategies. A computable execution strategy is a strategy for which a concrete implementation exists. Formally:

Definition 12.2 (computable strategy) A computable execution strategy $\xi$ is an execution strategy for which the following exist: a set of concrete states $\mathcal{C} \Sigma$, a computable ${ }^{1}$ abstraction function $\alpha: \mathcal{C} \Sigma \rightarrow \Sigma^{\mathrm{CHR}}$, a computable initialization function $\beta: \Sigma^{\text {init }} \rightarrow \mathcal{C} \Sigma$ such that $\forall \sigma \in \Sigma^{i n i t} \alpha(\beta(\sigma))=\sigma$, and a computable concrete transition function $\mathcal{C} t: \mathcal{C} \Sigma \rightarrow \mathcal{C} \Sigma$, such that, $\forall x \in \Sigma^{\text {init }}$ :

$$
x \rightsquigarrow \xi y \Longleftrightarrow \exists n \in \mathbb{N}: \alpha\left(\mathcal{C t}^{n}(\beta(x))\right)=y \text {, and } \mathcal{C} t^{n+1}(\beta(x)) \text { is undefined }
$$

In the above definition, the concrete states $\mathcal{C} \Sigma$ and the concrete transition function $\mathrm{Ct}$ correspond to the internal mechanism of a CHR implementation. The concrete states contain additional information, supplementing the abstract $\left(\omega_{t}\right)$ execution state. The concrete transition function is a function and not an arbitrary relation, i.e. the concrete state must contain enough information to determine a unique next state. The abstraction function $\alpha$ and the initialization function $\beta$ provide an interface between the concrete states and the abstract $\omega_{t}$ semantics.

\footnotetext{
${ }^{1} \mathrm{~A}$ function is computable if there is a Turing machine that computes it.
} 


\subsubsection{Strategy classes}

An execution strategy completely determines the result of any query. In general, although the result set may be smaller than that of all $\omega_{t}$ derivations, most CHR systems are still not completely deterministic. For instance, the refined operational semantics does not fix the order of constraint reactivation and partner constraint matching, which could lead to different results for the same query. However, a specific version of a CHR system, possibly with specific compiler optimizations turned on or off, should be completely deterministic, so it has only one execution strategy for every CHR program.

Definition 12.3 (strategy class) A strategy class $\Omega(\mathcal{P}) \subseteq \Omega_{t}^{\mathcal{H}}(\mathcal{P})$ is a set of execution strategies for $\mathcal{P}$.

Many CHR implementations are (possibly different) instantiations of the refined semantics, in the sense that their strategy class is a subset of $\Omega_{r}^{\mathcal{H}}$.

(1). For a specific version and configuration of the optimization flags, the Leuven CHR system corresponds to one execution strategy $\xi_{\mathrm{KUL}}$, which is an element of the strategy class corresponding to the refined semantics. We can say that (one version of) the Leuven CHR system corresponds to the singleton strategy class $\left\{\xi_{\mathrm{KUL}}\right\}$.

Note that strategy classes are subsets of the power set of $\Delta_{\omega_{t}}^{\mathcal{H}}$. We will sometimes drop the argument $(\mathcal{P})$ to avoid heavy notation.

Definition 12.4 (computable strategy class) A computable strategy class is a strategy class which contains at least one computable execution strategy.

Clearly, the Leuven CHR system strategy class $\left\{\xi_{\mathrm{KUL}}\right\}$ is computable, which implies that the refined semantics is also a computable strategy class, and so is the abstract semantics.

\section{Refined operational semantics $\omega_{r}$}

We define $\Sigma_{r}^{\mathrm{CHR}}$ to be the set of execution states of the refined semantics (see Section 4.3 on page 64$)$ and $\Omega_{r}^{\mathcal{H}}(\mathcal{P})$ as the strategy class corresponding to $\omega_{r}$ derivations of a program $\mathcal{P}$.

Not all execution strategies in $\Omega_{r}^{\mathcal{H}}(\mathcal{P})$ are computable: 
Example 45 (non-computable refined execution strategy). Consider the following program:

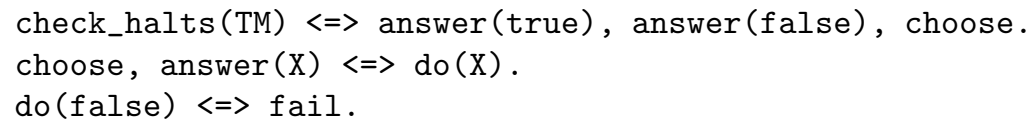

With an appropriate $\omega_{r}$ execution strategy, this program solves the halting problem. Since the second rule is applied with choose $/ 0$ as the active constraint, the refined semantics does not fix the choice of partner constraint. The execution strategy that always picks the correct answer is obviously not computable.

To show that $\Omega_{r}^{\mathcal{H}}(\mathcal{P})$ is a computable strategy class, it suffices to identify one computable execution strategy in $\Omega_{r}^{\mathcal{H}}(\mathcal{P})$. Following the definitions of Duck et al. (2004), we can define an abstraction function $\alpha$ which maps $\Sigma_{r}^{\mathrm{CHR}}$ to $\Sigma^{\mathrm{CHR}}$ :

$$
\alpha\left(\langle\mathbb{A}, \mathbb{S}, \mathbb{B}, \mathbb{T}\rangle_{n}\right)=\left\langle\operatorname{no} \_i d_{-}(\mathbb{A}), \mathbb{S}, \mathbb{B}, \mathbb{T}\right\rangle_{n}
$$

where no_id $(\mathbb{A})=\{c \mid c \in \mathbb{A}$ is not of the form $c \# i$ or $c \# i: j\}$. The set of concrete states is $\Sigma_{r}^{\mathrm{CHR}}$ and the initialization function is the identity function.

Now we still have not given a computable execution strategy, which needs to have a transition relation that is a computable function, that is, every state has a unique next state, and the latter can be computed from the former. The transition relation of $\omega_{r}$ is not a function, so we consider a subset of it which is a function, for instance, the function which maps every execution state in $\sigma \in \Sigma_{r}^{\mathrm{CHR}}$ to the lexicographically first element in $\left\{\sigma^{\prime} \mid \sigma \varlimsup^{\omega_{r}} \sigma^{\prime}\right\}$. Since the set of all next $\omega_{r}$ states is computable, and the lexicographically first element of a set is computable, this function is computable.

\section{Priority semantics $\omega_{p}$}

We define $\Omega_{p}^{\mathcal{H}}(\mathcal{P})$ as the strategy class corresponding to derivations in the priority semantics $\omega_{p}$ (see Section 4.1.4 on page 60) We denote an assignments of priorities to rules with $\bar{p}$, and we write $\Omega_{p}^{\mathcal{H}}(\mathcal{P}, \bar{p})$ for the subset of $\Omega_{p}^{\mathcal{H}}(\mathcal{P})$ which corresponds to $\omega_{p}$ derivations with the priority assignments $\bar{p}$.

Again, $\Omega_{p}^{\mathcal{H}}(\mathcal{P})$ contains non-computable execution strategies as well as computable ones.

Figure 12.1 shows some execution strategies and strategy classes.

(1) The execution strategy "dPCHR" is that of an implementation of probabilistic CHR (Frühwirth, Di Pierro, and Wiklicky 2002), in which all rules get the same probability and the rules are picked using a deterministic pseudo-random number generator initialized with some fixed seed. This execution strategy does not belong to $\Omega_{r}^{\mathcal{H}}(\mathcal{P})$, nor does it belong to $\Omega_{p}^{\mathcal{H}}(\mathcal{P})$. 


\subsubsection{Generalized confluence}

We now generalize the definition of confluence to arbitrary strategy classes:

Definition 12.5 ( $\Omega$-confluence) A CHR program $\mathcal{P}$ is $\Omega(\mathcal{P})$-confluent if, for every initial state $\langle\mathbb{G}, \emptyset \text {, true, } \emptyset\rangle_{1}=\sigma \in \Sigma^{\text {init }}$ and arbitrary execution strategies $\xi_{1}, \xi_{2} \in \Omega(\mathcal{P})$, the following holds: if $\sigma \rightsquigarrow \xi_{1} \sigma_{1}$ and $\sigma \rightsquigarrow \xi_{2} \sigma_{2}$, then $\sigma_{1} \approx \sigma_{2}$.

Note that $\Omega_{t}^{\mathcal{H}}(\mathcal{P})$-confluence corresponds to the usual confluence, while $\Omega_{r}^{\mathcal{H}}(\mathcal{P})$ confluence corresponds to "confluent in the refined semantics" - see chapter 6 of (Duck 2005) for more about the latter. If the strategy class $\Omega$ is a singleton, every CHR program $\mathcal{P}$ is trivially $\Omega$-confluent.

Actually, in the above definition we require $\sigma$ to be an initial state, while in the usual definitions of confluence (e.g. that of Abdennadher et al. (1999)), this is not the case. As a result, there is a slight difference between $\Omega_{t}^{\mathcal{H}}(\mathcal{P})$-confluence and the usual confluence. It is possible that a program is $\Omega_{t}^{\mathcal{H}}(\mathcal{P})$-confluent while there is a state (that is not an initial state and cannot be reached from any initial state) from which there are two different derivations to final states that are not variants of each other. This distinction between confluence and reachable confluence is discussed in (Duck, Stuckey, and Sulzmann 2007). In the opinion of the author, these examples - programs that are R-confluent (which corresponds to $\Omega_{t}^{\mathcal{H}}(\mathcal{P})$-confluent) but not confluent - indicate a flaw in the original definition of confluence.

Figure 12.2 shows sets of $\Omega$-confluent $\mathrm{CHR}$ programs corresponding to the strategy classes in Figure 12.1.

We have the following duality property that follows directly from the definitions: for all strategy classes $\Omega_{1}$ and $\Omega_{2}$ : if $\Omega_{1} \subseteq \Omega_{2}$, then every $\Omega_{2}$-confluent program is also $\Omega_{1}$-confluent. In other words, if $\Omega_{2}$ is more general than $\Omega_{1}$ (i.e. it allows more derivations), then $\Omega_{2}$-confluence is stronger than $\Omega_{1}$-confluence.

\subsection{General CHR machines}

We generalize the deterministic abstract CHR machines of Chapter 10 as follows:

Definition 12.6 (general CHR machine) A (general) CHR machine is a tuple $\mathcal{M}=(\mathcal{H}, \Omega, \mathcal{P}, \mathcal{V G})$ where the host language $\mathcal{H}$ defines a built-in constraint theory $\mathcal{D}_{\mathcal{H}}, \mathcal{P}$ is a $C H R$ program, $\mathcal{L G} \subseteq \mathcal{G}_{\mathcal{P}}^{\mathcal{H}}$ is a set of valid goals, and $\Omega \subseteq \Omega_{t}^{\mathcal{H}}(\mathcal{P})$ is a strategy class. The machine takes an input query $\mathbb{G} \in \mathcal{V} \mathcal{G}$, picks any execution strategy $\xi \in \Omega$, and executes the derivation $\left.d \in \xi\right|_{\mathbb{G}}$.

Note that we no longer require the program to be $\Delta_{\omega_{t}}^{\mathcal{H}}$-deterministic for valid input, and we allow the machine to use any strategy class. 


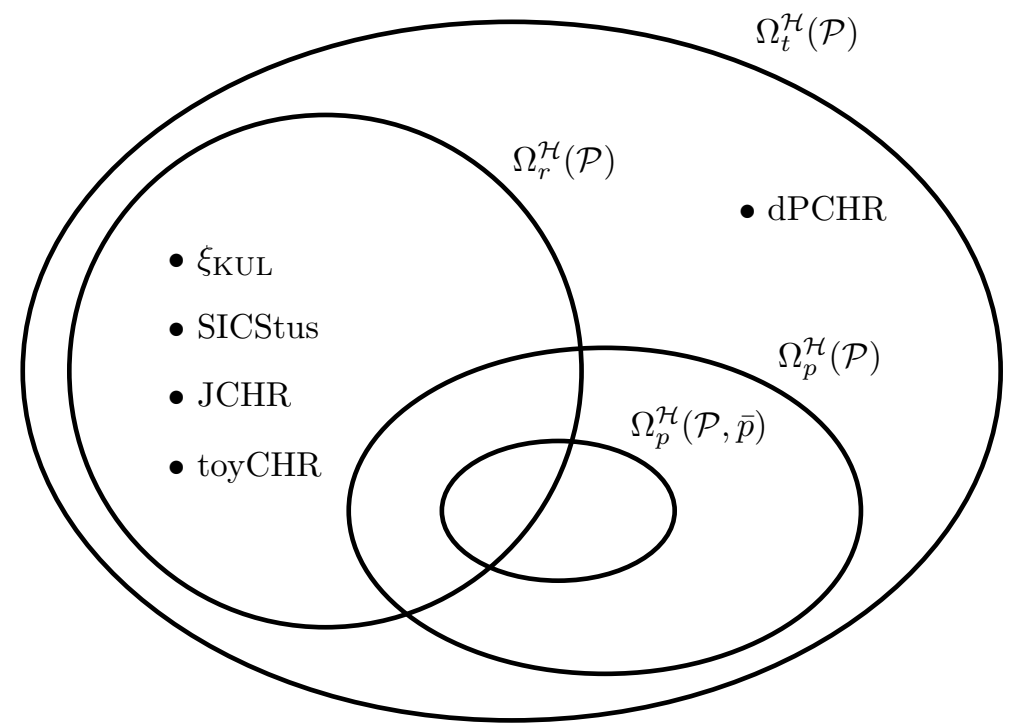

Figure 12.1: Execution strategies

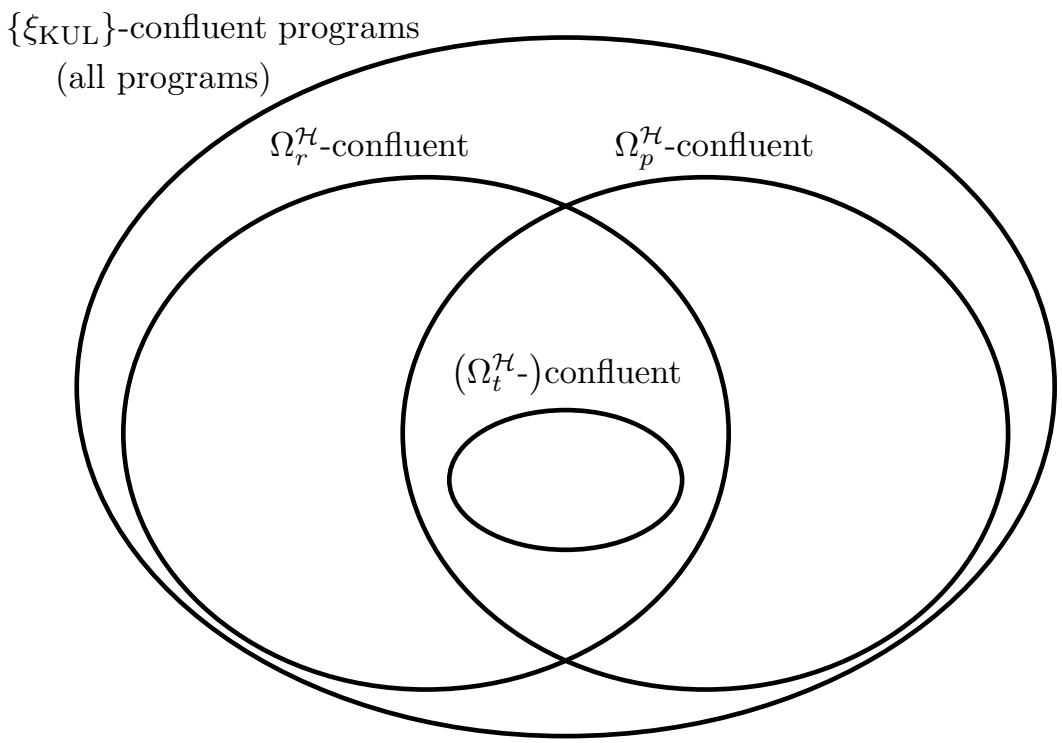

Figure 12.2: Generalized confluence 


\subsubsection{Terminology}

A $C H R(\mathcal{H})$ machine is a CHR machine of the form $\left(\mathcal{H},{ }_{-},-,-\right)$. An $\Omega$-CHR machine is a CHR machine of the form $\left(-, \Omega_{-},-\right)$. Abstract CHR machines are $\Omega_{t}^{\mathcal{H}}-\mathrm{CHR}$ machines, and refined CHR machines are $\Omega_{r}^{\mathcal{H}}$-CHR machines. A feasible CHR machine is one with a computable strategy class. A confluent CHR machine $\left({ }_{-}, \Omega, \mathcal{P},_{-}\right)$has a program $\mathcal{P}$ which is $\Omega$-confluent. A deterministic CHR machine $\left({ }_{-}, \Omega, \mathcal{P},{ }_{-}\right)$has a program $\mathcal{P}$ which is $\Delta_{\Omega}$-deterministic, where $\Delta_{\Omega}=\bigcup \Omega(\mathcal{P})$ is the set of all possible derivations.

\subsubsection{Complexity of general CHR machines}

We generalize the time and space functions of Section 10.4 in the obvious way:

Definition 12.7 (generalized time function) Given an $\Omega$-CHR machine $\mathcal{M}$, the time function chrtime $\mathcal{M}$ returns the worst-case derivation length:

$$
\text { chrtime }_{\mathcal{M}}(\mathbb{G})=\max \left\{\# d|\exists \xi \in \Omega: d \in \xi|_{\mathbb{G}}\right\}
$$

Definition 12.8 (generalized space function) Given an $\Omega$-CHR machine $\mathcal{M}$, the space function chrspace $\mathcal{M}_{\mathcal{M}}$ returns the worst-case execution state size:

$$
\text { chrspace }_{\mathcal{M}}(\mathbb{G})=\max \left\{\operatorname{SIZE}(\sigma)|\exists \xi \in \Omega: \sigma \in \in \xi|_{\mathbb{G}}\right\}
$$

It is not clear precisely what the added power is of generalized CHR machines compared to abstract CHR machines. For well-known strategy classes, like $\Omega_{r}^{\mathcal{H}}$ or $\Omega_{p}^{\mathcal{H}}$, we can still only decide the languages in $\mathrm{P}$ in polynomial time. However, it seems that such more instantiated strategy classes do add some power. For example, in the refined semantics, we can (non-monotonically) check for absence of constraints, and in the priority semantics, we can easily sort in linear time.

Of course, a CHR machine with a strategy class that is not computable can be arbitrarily strong - any oracle can be formulated as an execution strategy; for example an oracle that decides the halting problem.

- More exotic strategy classes can be conceived, that could still be computable while implicitly requiring more than a polynomial amount of work to compute the next transition. For such strategy classes, the corresponding generalized CHR machine could clearly be much more powerful than an abstract CHR machine.

\subsection{Non-deterministic CHR machines}

We define non-deterministic CHR machines similarly to the way non-deterministic Turing machines are defined (cf. Section 2.1.2). 
Listing 12.1: 3SAT: NCHR program to solve 3SAT clauses

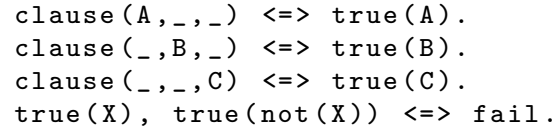

Definition 12.9 (NCHR machine) A non-deterministic CHR machine (NCHR machine) is a tuple $\mathcal{M}=(\mathcal{H}, \Omega, \mathcal{P}, \mathcal{L G})$, where $\mathcal{H}, \Omega, \mathcal{P}$, and $\mathcal{L G}$ are defined as before. The machine takes an input query $\mathbb{G} \in \mathcal{V G}$ and considers all execution strategies $\xi \in \Omega$. If there are strategies that result in a successful derivation $\left.d \in \xi\right|_{\mathbb{G}}$, any of those is returned. Otherwise, any failure derivation is returned. If all derivations are infinite, any infinite derivation is returned.

As an example, consider the program 3SAT in Listing 12.1. The NCHR machine $\left(\emptyset, \Omega_{t}^{\mathcal{H}}, 3 S A T, 3\right.$ SATCLAUSES) decides 3SAT clauses in linear time. A 3SAT clause of the form $\left(x_{1} \vee x_{2} \vee x_{3}\right) \wedge\left(y_{1} \vee y_{2} \vee y_{3}\right) \wedge \ldots$ is encoded as a query clause $(\mathrm{x} 1, \mathrm{x} 2, \mathrm{x} 3)$, clause $(\mathrm{y} 1, \mathrm{y} 2, \mathrm{y} 3), \ldots$, where negative literals are encoded by wrapping them in not/1. The valid goals 3SATCLAUSES (i.e. admissible input clauses, but not necessarily satisfiable) are all goals of this form.

In every derivation, all clause/ 3 constraints are simplified, so one of the literals has been made true. If the instance of 3SAT has a solution, there is a way to do this without producing a conflicting truth assignment, so there is a successful derivation. If there is no solution, all derivations will fail because every assignment causes a conflict, which causes the fourth rule to fire.

The NCHR machine $\left(\emptyset, \Omega_{r}^{\mathcal{H}}, 3\right.$ SAT, 3SATCLAUSES $)$ - the same as above but with its strategy class restricted to the refined semantics - is no longer correct. Because execution strategies are limited to those of the $\omega_{r}$ semantics, every clause/3 constraint will be simplified by the first rule. As a result, the only truth assignment that is tried is to make every first literal of all clauses true.

Non-determinism in rule choice is exploited in the program 3SAT. However, we can easily shift the non-determinism to the choice of matching partner constraints for an active constraint. For example, the following program, when executed on a refined NCHR machine, decides 3SAT clauses in linear time:

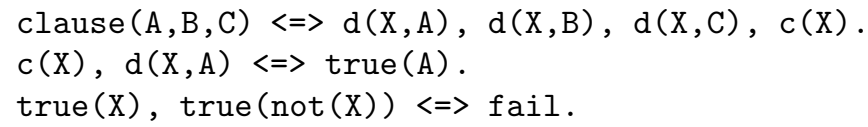

However, as a general rule, the smaller the strategy class, the harder it is to write a correct NCHR program: when there are less sources of non-determinism, the corresponding NCHR machine becomes less powerful. When the strategy class is a singleton, there is of course no difference between a regular CHR machine and an NCHR machine. 
For regular CHR machines, the reverse rule of thumb holds: the larger the strategy class, the harder it is to write a correct CHR program - more nondeterminism only means more wrong choices. If we denote the class of decision problems that can be solved by a deterministic $\Omega$-CHR machine in polynomial time with $\mathbf{P}_{\Omega}$, and the class of decision problems that can be solved by a polynomialtime non-deterministic $\Omega$-CHR machine with $\mathbf{N P}_{\Omega}$, then we have the following inclusions:

$$
\mathbf{P}_{\Omega_{t}^{\mathcal{H}}} \subseteq \mathbf{P}_{\Omega_{r}^{\mathcal{H}}} \subseteq \mathbf{P}_{\left\{\xi_{\mathrm{KUL}}\right\}}=\mathbf{N P}_{\left\{\xi_{\mathrm{KUL}}\right\}} \subseteq \mathbf{N P}_{\Omega_{r}^{\mathcal{H}}} \subseteq \mathbf{N P}_{\Omega_{t}^{\mathcal{H}}}
$$

Most of these inclusions collapse to equalities: since the RAM simulator program RAMSIMUL (see Listing A.2 on page 221 ) is $\left(\Omega_{t}^{\mathcal{H}}-\right.$ ) confluent, we have:

$$
\mathbf{P}_{\Omega_{t}^{\mathcal{H}}}=\mathbf{P}_{\left\{\xi_{\mathrm{KUL}}\right\}}
$$

(1) In this sense, the strategy class does not seem to affect the computational power of the CHR machine. Still, it is our experience that it is easier to write programs for a more instantiated operational semantics. In the case of the self-modifying CHR machines of the next section, it does seem to be the case that instantiating the operational semantics really adds power to the machine.

The class of languages that are decided in polynomial time by a non-deterministic CHR machine (refined or abstract) coincides with NP. We only prove the inclusion $\mathbf{N P} \subseteq \mathbf{N P}_{\Omega_{r}^{\mathcal{H}}}$ :

Theorem 12.10 The non-deterministic refined CHR-only machine can simulate a non-deterministic Turing machine with the same asymptotic time complexity.

Proof. The simulator program TMSIM (Listing 10.1 on page 167) also works if $\operatorname{delta} / 5$ is not a function but defines more than one transition for a given state and symbol. The non-determinism in choosing the partner constraints of head/1 (the computation-driving active constraint) ensures that all Turing machine computation paths are simulated.

In the above, the word "refined" can also be replaced by "abstract". So in summary, we have:

$$
\mathbf{N P}=\mathbf{N P}_{\Omega_{r}^{\mathcal{H}}}=\mathbf{N P}_{\Omega_{t}^{\mathcal{H}}}
$$

\subsection{Self-modifying CHR machines}

Section 2.1.5 dealt with the RASP machine (random access stored program), a variant of the RAM machine which can access and alter its own program instructions since instructions and data are stored in the same memory space. In terms 


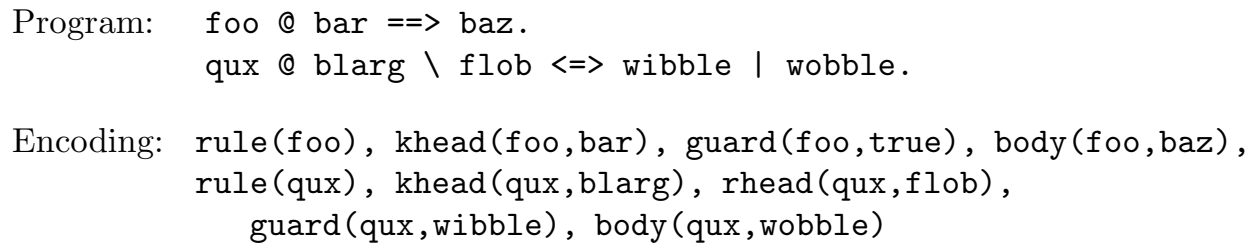

Figure 12.3: Example encoding of a CHR program for CHRSP machines

3. Apply. $\left\langle\mathbb{G}, H_{1} \cup H_{2} \cup \mathbb{S}, \mathbb{B}, \mathbb{T}\right\rangle_{n} \longmapsto_{\omega_{t}^{s p}, \mathcal{P}}\left\langle C^{\prime} \uplus \mathbb{G}, H_{1} \cup \mathbb{S}, \theta \wedge \mathbb{B}, \mathbb{T} \cup\{h\}\right\rangle_{n}$ where $\operatorname{chr}(\mathbb{S})$ contains the following constraints, which encode a rule: $\operatorname{rule}(r), \operatorname{khead}\left(r, h_{1}\right), \ldots, \operatorname{khead}\left(r, h_{k}\right), \operatorname{rhead}\left(r, h_{k+1}\right), \ldots$, $\operatorname{rhead}\left(r, h_{l}\right), \operatorname{guard}(r, g)$, body $(r, C)$, and neither $\mathbb{G}$ nor $\mathbb{S}$ contain any other rule-encoding constraints with $r$ as a first argument. Now let $g^{\prime}, C^{\prime}$, $H_{1}^{\prime}$, and $H_{2}^{\prime}$ be consistently renamed apart versions of $g, C,\left(h_{1} \wedge \cdots \wedge h_{k}\right)$, and $\left(h_{k+1} \wedge \cdots \wedge h_{l}\right)$, respectively. This encoding corresponds to a rule of the form $r @ H_{1}^{\prime} \backslash H_{2}^{\prime} \Longleftrightarrow g^{\prime} \mid C^{\prime}$. As usual, $\theta$ is a matching substitution such that $\operatorname{chr}\left(H_{1}\right)=\theta\left(H_{1}^{\prime}\right)$ and $\operatorname{chr}\left(H_{2}\right)=\theta\left(H_{2}^{\prime}\right)$ and $h=\left(r, i d\left(H_{1}\right), i d\left(H_{2}\right)\right) \notin T$ and $\mathcal{D}_{\mathcal{H}}=\left(\bar{\exists}_{\emptyset} \mathbb{B}\right) \wedge\left(\mathbb{B} \rightarrow \bar{\exists}_{\mathbb{B}}\left(\theta \wedge g^{\prime}\right)\right)$.

Figure 12.4: Modified Apply transition in the $\omega_{t}^{s p}$ semantics

of computational power and complexity, the RASP machine is just as powerful as a regular RAM machine; the reason is that a RASP simulator can be implemented on a RAM machine, with only constant time and space overhead.

In this section, we examine CHR machines with a stored program, or CHRSP machines. Since the CHR program is now stored in the CHR store, it can be accessed and modified like any other CHR constraints.

\subsubsection{Definition}

We use a syntax that somewhat resembles that of (Frühwirth and Holzbaur 2003), where it was proposed in the context of source-to-source transformation. A CHR program is encoded using the following 'reserved keyword' constraints: rule/1, khead $/ 2, \operatorname{rhead} / 2, \operatorname{guard} / 2$, and body/2. To illustrate the encoding, Figure 12.3 shows a CHR program and the corresponding constraint representation.

Now, we modify the Apply transition of $\omega_{t}$ to refer to the stored program as in Figure 12.4. We call the resulting semantics the abstract stored-program semantics $\omega_{t}^{s p}$. Note that because the program is now in the constraint store, we no longer need to (implicitly) parametrize the semantics with a CHR program; the program is now part of the query or, equivalently, corresponds to the initial store. 
Listing 12.2: HAMILTON: CHRSP program to find Hamiltonian paths

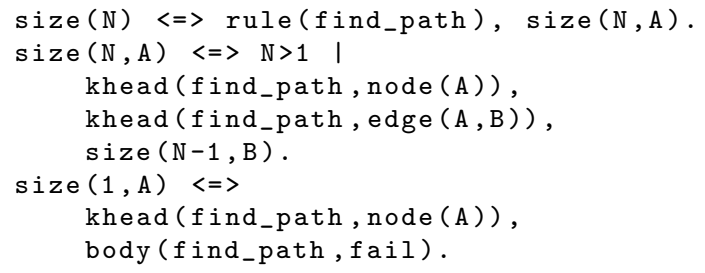

In order to avoid premature application of rules, i.e. firing a rule which is still being constructed, we require that a rule can only fire if there are none of its components in the goal, waiting to be introduced into the store.

A CHRSP machine is defined just like a regular CHR machine, except that the operational semantics is altered in the way described above. Also, if a program is given for CHRSP machines, it should be considered to be an abbreviation for the encoded form, which is implicitly appended to all valid goals. As before, we define every $\omega_{t}^{s p}$ transition to take constant time; the machine rejects the input if the final state is a failure state, otherwise it accepts or does not terminate.

\subsubsection{Complexity of CHRSP machines}

Unlike RASP machines, which can be efficiently simulated on RAM machines, CHRSP machines cannot be simulated on regular CHR machines with only constant overhead. The reason is that finding $k$ partner constraints in a store of size $n$ can take $O\left(n^{k}\right)$ time. For a fixed program, $k$ is bounded, but on a CHRSP machine, rules with an arbitrary number of heads may be created.

In fact, self-modifying CHR programs can decide co-NP-complete languages in linear time. Consider the problem of Hamiltonian paths in directed graphs. Deciding whether a graph has a Hamiltonian path is NP-complete, so the language consisting of all graphs that do not have a Hamiltonian path is co-NP-complete. Now consider the self-modifying CHR program HAMILTON of Listing 12.2

As input query, we encode a graph in the following way: edge/2 constraints for the edges; node/ 1 constraints for the $n$ nodes; one size $(n)$ constraint to indicate the number of nodes. The program creates a rule of the form

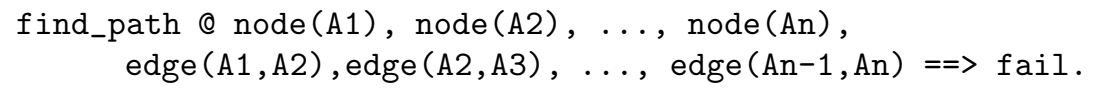

If the graph has a Hamiltonian path, this rule fires and the CHRSP machine rejects the input. Otherwise, the machine accepts the input. Either way, the machine halts and takes only linear time and space. 
If a regular CHR machine exists that can simulate CHRSP machines with only polynomial overhead, then co-NP $\subseteq \mathbf{P}$, and thus $\mathbf{P}=\mathbf{N P}$. So if $\mathbf{P} \neq \mathbf{N P}$, CHRSP machines are strictly more powerful than regular CHR machines.

\subsection{Summary and conclusion}

We have defined three different generalizations of the deterministic abstract CHR machine of Chapter 10: general CHR machines with restricted strategy classes, non-deterministic CHR machines, and stored-program (self-modifying) CHR machines. We have investigated the complexity properties of these generalized CHR machines.

The generalizations are orthogonal, so they can be combined. Indeed, one could very well consider, for instance, a refined self-modifying CHR machine.

\subsubsection{Complexity summary}

As demonstrated by the program TMSIM (see Listing 10.1 on page 167), a regular CHR machine can do in polynomial time what a Turing machine (or a RAM machine) can do in polynomial time:

$$
\mathbf{P}_{\Omega_{t}}=\mathbf{P}
$$

In Section 12.3 we showed a similar result for non-deterministic CHR machines:

$$
\mathbf{N P}_{\Omega_{t}}=\mathbf{N P}
$$

However, although $\mathbf{P}_{\mathrm{RASP}}=\mathbf{P}$, self-modifying CHR machines are more powerful than regular ones, although exact bounds are still an open problem:

$$
\mathbf{c o - N P} \subseteq \mathbf{P}_{\Omega_{t}^{s p}} \subseteq \text { PSPACE }
$$

Restricting the strategy class to an instantiation of $\Omega_{t}$ (or $\Omega_{t}^{s p}$ ) can make the CHR machine stronger: a self-modifying refined CHR machine can also solve NPcomplete problems in linear time by checking for absence of a solution to the corresponding co-NP problem. Checking for absence is not known to be possible in the abstract semantics. So $\mathbf{P}_{\Omega_{t}^{s p}} \subseteq \mathbf{P}_{\Omega_{r}^{s p}}$ (and we conjecture the inclusion to be strict), and also co-NP $\cup \mathbf{N P} \subseteq \mathbf{P}_{\Omega_{r}^{s p}}$.

\subsubsection{Future work}

Determining the exact complexity classes corresponding to CHRSP machines is still an open problem. It is also not clear to what extent the choice of strategy class influences the computational power, even for regular generalized CHR machines, let alone in the case of non-deterministic and/or stored-program CHR machines. 


\section{Chapter 13}

\section{Conclusion}

"Go on, get out. Last words are for fools who haven't said enough."

- Karl Marx (1818-1883), last words

"We can only see a short distance ahead, but we can see plenty there that needs to be done."

- Alan Turing (1912-1954)

In this final chapter we conclude with a brief summary of our contributions, followed by a discussion of potential directions for future research.

\subsection{Contributions}

In Section 1.2 we have stated our main research goal: to demonstrate and enhance the usability of CHR as a high-level general-purpose programming language by studying and improving its performance, both in theory and in practice. We now briefly summarize how each chapter contributed towards this goal.

Chapters 2-4 introduce background material related to computational complexity theory, the CHR language, and CHR compilation. As such, these chapters do not discuss novel contributions. In Chapter 5 we construct two new CHR implementations of classic algorithms - Dijkstra's shortest path algorithm and Hopcroft's DFA minimization algorithm. The contribution of this chapter is that it adds to the body of empirical evidence for the applicability of CHR to generalpurpose programming.

\section{Optimizing compilation of CHR}

Part II contributes a number of new compiler optimizations, improving the performance of CHR systems. Moreover, all these optimizations are of crucial im- 
portance to achieve the complexity-wise completeness result of Part III, with the exception of the guard reasoning optimizations of Chapter 6. Most of the indexing techniques of Chapter 7 were introduced by others; the novel contributions of that chapter are dense_int indexing and the concept of guard solving. Chapter 8 contributes two new optimizations aimed at improving memory usage - as far as we know these are the first optimizations specifically aimed at improving the space complexity of CHR. The main contribution of Chapter 9 is of a theoretical nature: while the other optimizations of Part II have been implemented and experimentally evaluated, Chapter 9 is limited to a detailed theoretical investigation of the join ordering problem for CHR.

\section{Computability and complexity of CHR}

While Part II deals with improving the performance of CHR in practice, Part III studies the computational complexity of CHR both in theory and in practice. Chapter 10 introduces the CHR machine and shows that it is Turing-complete. In Section 10.3, Turing-complete and non-Turing-complete subsets of CHR are identified, which provides a way to classify language features as essential or redundant from a computational perspective.

The core results of Part II are shown in Chapter 11: a complexity meta-theorem that is much more accurate than earlier work, and a complexity-wise completeness result for CHR: any algorithm can be implemented in CHR and executed with the optimal time and space complexity (in a state-of-the-art CHR system). In Section 11.4 we attempt to 'port' the complexity-wise completeness result to other declarative languages. The results indicate that $\mathrm{CHR}$ is in a relatively privileged position compared to other declarative languages. The contribution of Section 11.5 is to consider the constant factors hidden behind the notion of asymptotic complexity. Based on an experimental evaluation of some case studies, we conjecture that the constant factor separating $\mathrm{CHR}$ (hProlog) from $\mathrm{C}$ is about one order of magnitude for the time complexity and less than an order of magnitude for space.

Finally, in Chapter 12 we introduce the notions of execution strategies and generalized confluence, and we investigate several variants of the CHR machine: general CHR machines, non-deterministic CHR machines, and self-modifying CHR machines.

\subsection{Future work}

Many of the chapters end by discussing possibilities for future work. These are often of a rather technical nature, or they are relatively incremental potential improvements. In this section we list some more general and long-term possibilities for future research. 


\subsubsection{Grand challenges for CHR}

In a recent survey (Sneyers, Van Weert, Schrijvers, and De Koninck 2008) we have identified four broad and largely open research topics, or grand challenges, that in our opinion should be addressed by the CHR research community. The topics are interesting from an academic point of view, but perhaps more importantly, they are also crucial for allowing a wide-spread adoption of the CHR programming language and the further creation of a large and lively CHR user community.

These are the four grand challenges:

1. Programming environments and tools. If measured by current standards, which dictate that a language is only as good as its tools, CHR is a poor language indeed. While several strong theoretical results have been obtained in the field of program analysis for CHR, little (if any) effort has been made to embody these results into practical tools for day-to-day programming. For example, programmers have to manually check for confluence ${ }^{1}$, and, in the case of non-confluence, complete their solvers by hand. Current debugging tools for CHR are rather rudimentary. Finally, there are still plenty of programming languages for which no CHR system has been constructed so far, especially among the imperative programming languages. At the moment, CHR systems are implemented from scratch for each host language. The development of a bootstrapping CHR compiler could dramatically improve the portability of CHR systems and new compiler optimizations.

2. Execution control. Compared to the refined operational semantics, the rule priorities of the $\mathrm{CHR}^{\mathrm{rp}}$ semantics are an important step in the realization of Kowalski's slogan "Algorithm = Logic + Control" (Kowalski 1979) in the context of CHR. However, there are still many remaining challenges in finding satisfying ways to allow programmers to fine-tune the execution strategy (control) of their programs.

3. Parallelism, concurrency. Recent theoretical work (Frühwirth 2005b; Meister 2006) confirms CHR's inherent aptness for parallel programming. However, to enable CHR programs to fully exploit the power of current and future multi-core processors, practical and efficient concurrent implementations are required. Currently, these implementations are still in early stages (see Section 3.3.2 for a discussion of some early Haskell-based prototypes).

\footnotetext{
${ }^{1}$ While automatic confluence tests have been described in the literature (see Section 3.2.1), there are no (polished) implementations available of a confluence checker as far as we know. Also, many instances of non-confluence are caused by considering queries for which the program is not intended to be used, or by different solutions for a query that are semantically equivalent but syntactically different. Formalisms for expressing the set of valid queries and the equivalence classes of solutions would be very useful to improve the accuracy of confluence tests (as well as many compiler optimizations).
} 
Many topics are still to be researched in this domain, including language features and semantics, analysis, implementation, and optimization.

4. Scalability. Current CHR systems are still essentially academic prototypes that have been tested well on small toy examples. However, in real industrial applications, the size of programs and input data sets is typically much larger. The refined semantics compilation scheme (see Chapter 4) was not designed or benchmarked with such program sizes in mind. Many scalability issues are beginning to surface, from the point of view of the human programmer as well as that of CHR system performance. Some potential aspects of scalability are the following:

- huge constraint stores that have to be persistent and/or distributed;

- (dynamic) optimizations, also for variants and extensions of CHR;

- incremental compilation, run-time rule assertion, reflection;

- higher-order / meta-programming;

- modularity, solver hierarchies, standardized libraries.

\subsubsection{Beyond CHR}

In CHR-related research, one of the major recent trends is to investigate the link between CHR and other formalisms. In Section 3.7 we gave a non-exhaustive overview; most of the work we referred to in that section is very recent and still ongoing. Establishing strong connections between CHR and other formalisms is only the first step towards a potentially very fruitful cross-fertilization of concepts and results. Since CHR seems to subsume most of the other formalisms that have been related to it, it has the potential to become a lingua franca, a hub which collects and dispenses research efforts from and to the various related fields.

There is no reason why the quest for increasingly higher level programming languages should end with CHR. There has already been some work on extensions of CHR that improve its expressivity (see Section 3.4). Perhaps further contribitions along those lines, combined with ideas from related high-level formalisms, will lead to an entirely new programming language that surpasses CHR. 


\section{Appendix A}

\section{RAM Machine Simulators}

This appendix contains program listings of the RAM machine simulators discussed in Chapter 11.

Listing A.1: PARAMSIM: Simulator of Peano-arithmetic RAM machines

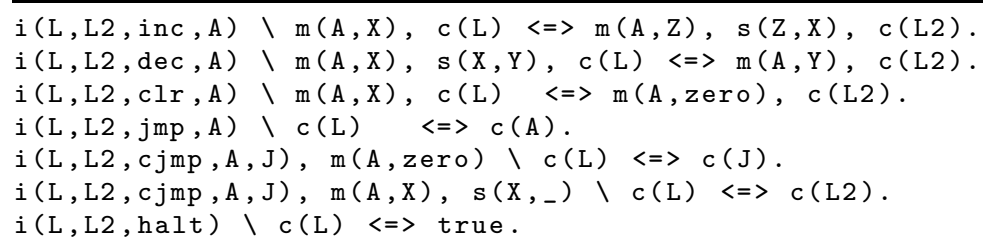

Listing A.2: RAMSIMUL: Simulator of standard RAM machines

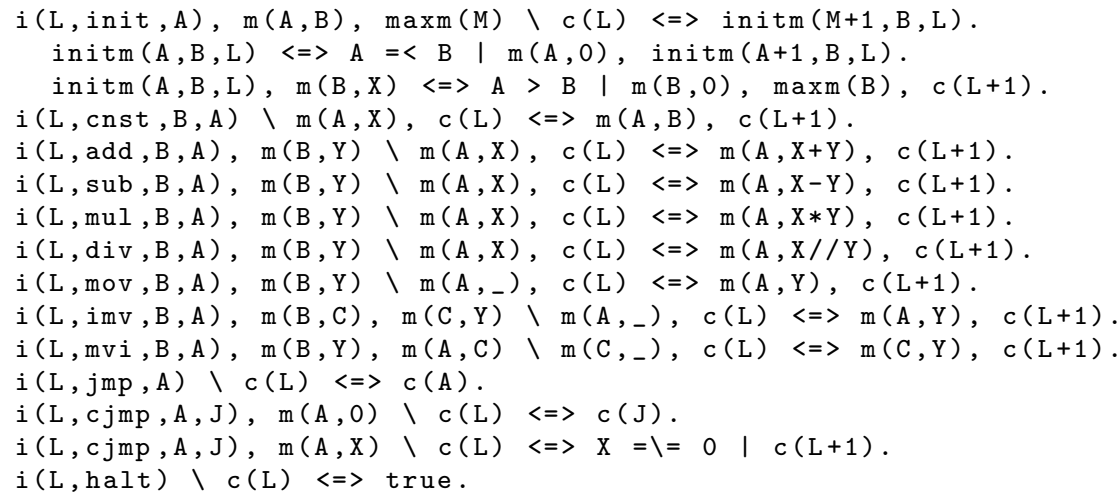


Listing A.3: Simulator of RAM machines, written in pure Prolog

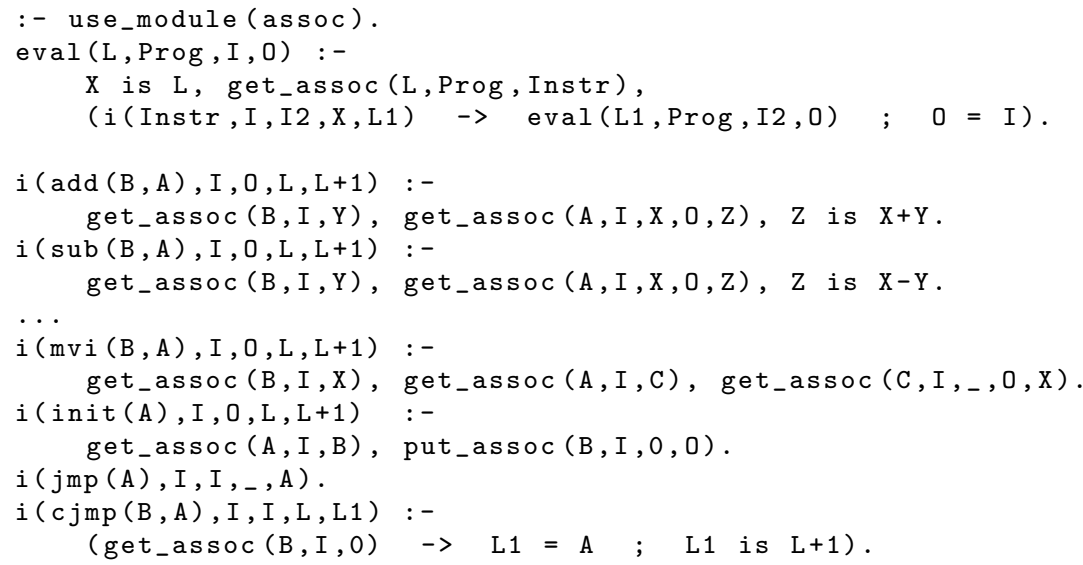

Listing A.4: Simulator of RAM machines, written in Haskell

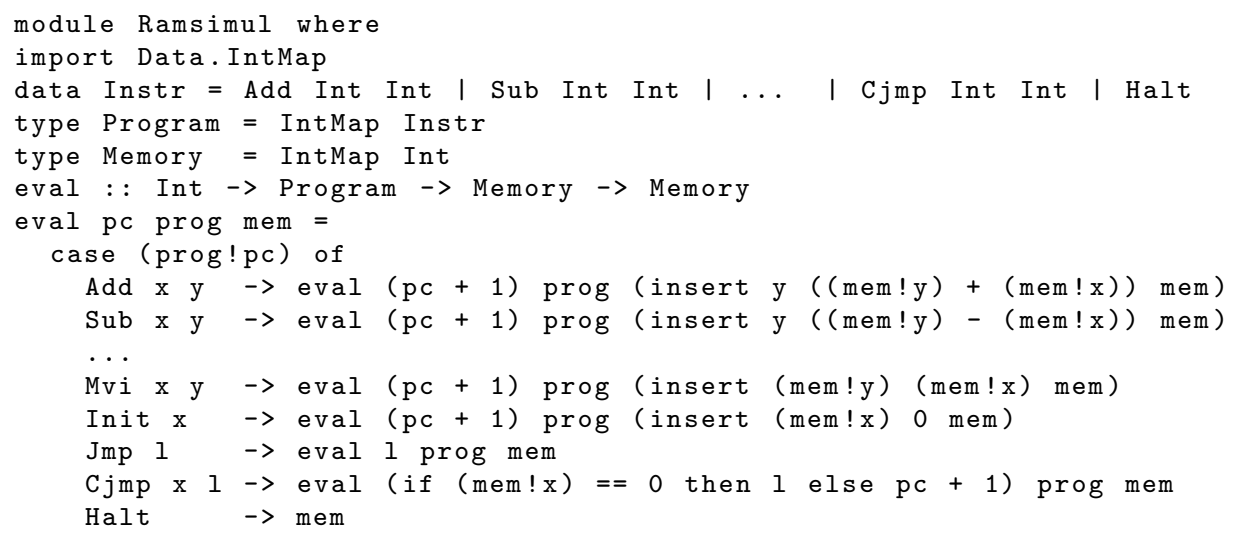


Listing A.5: Simulator of RAM machines, written in Maude

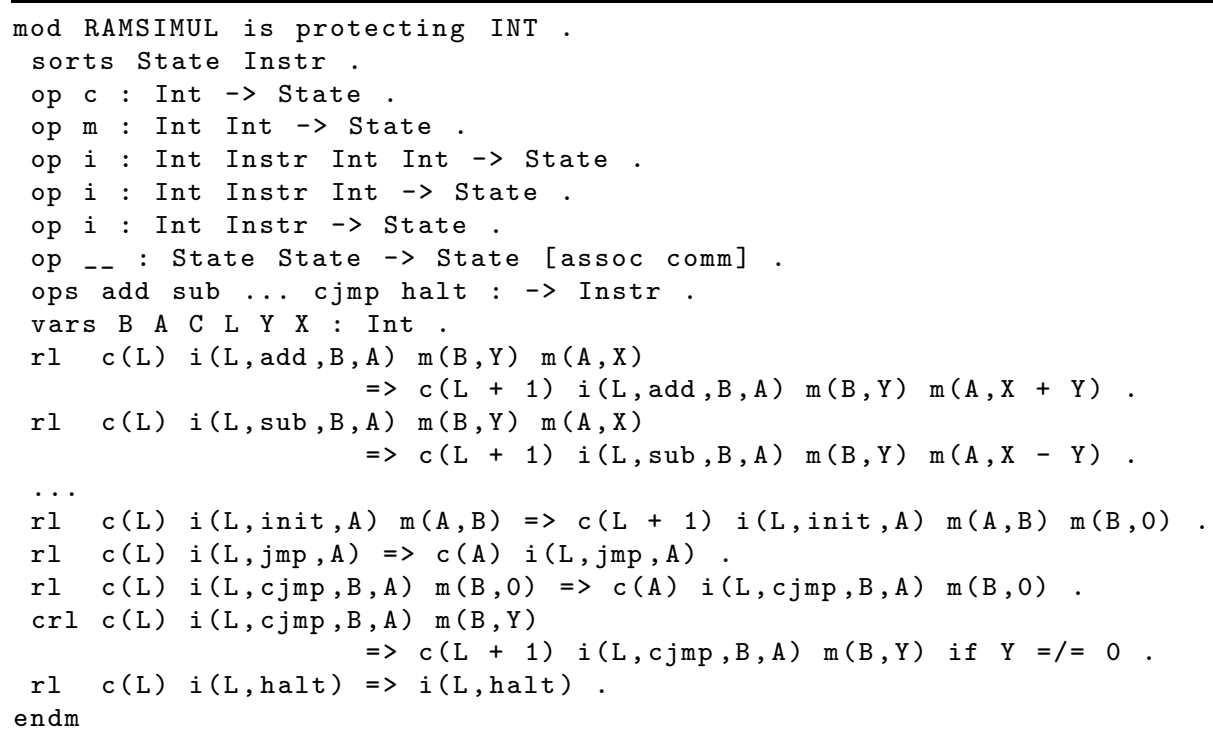

Listing A.6: More efficient RAM simulator in Maude, using Map

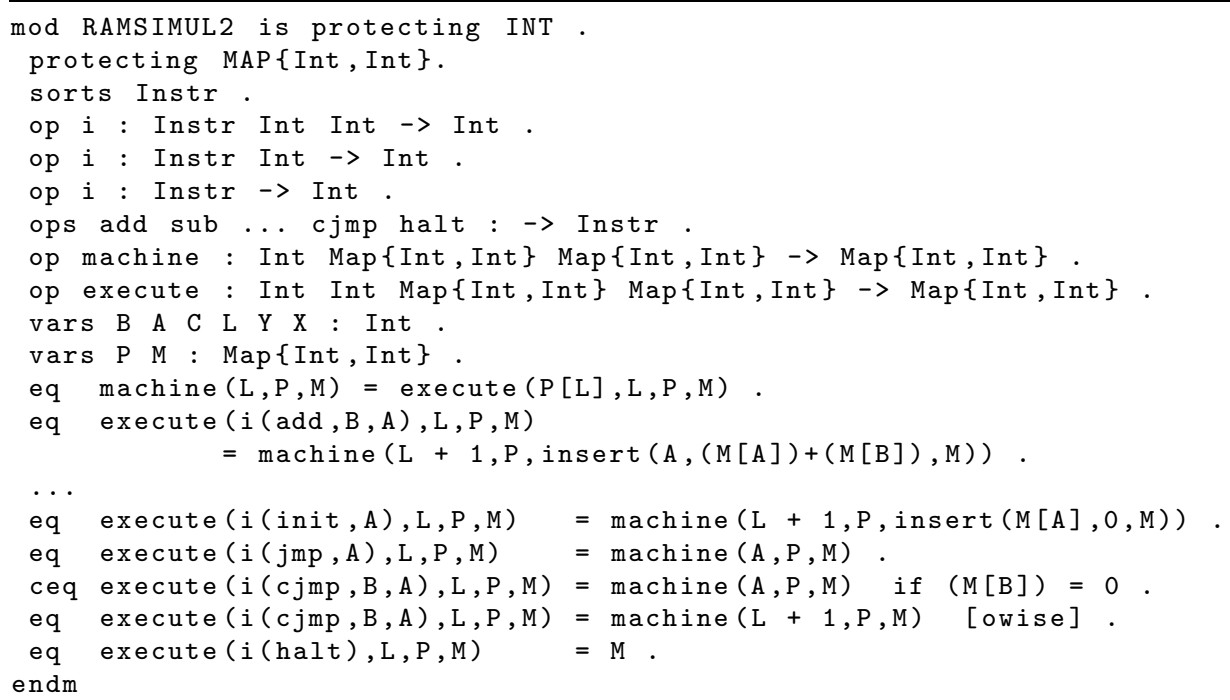


Listing A.7: Simulator of RAM machines, written in Jess

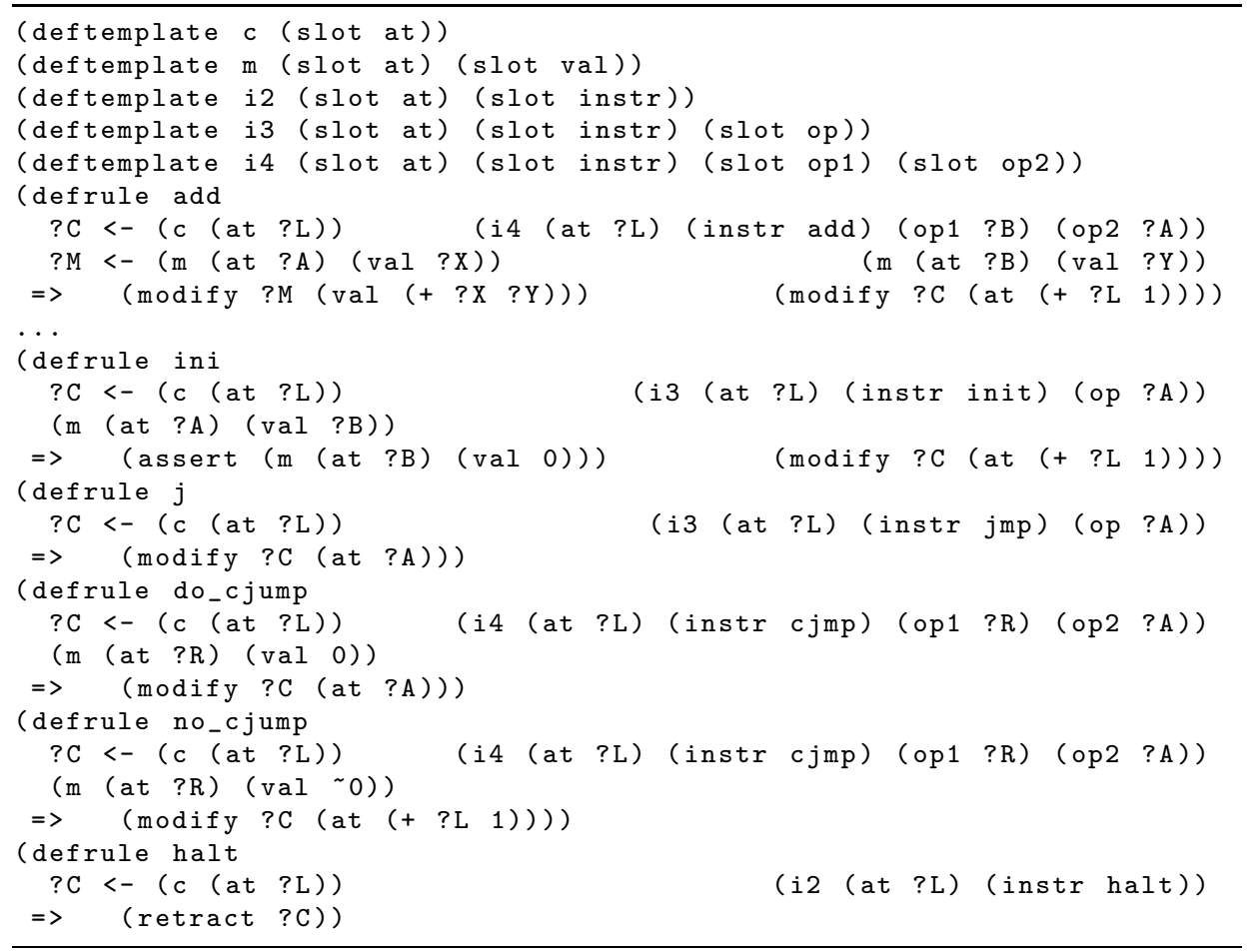




\section{Appendix B}

\section{Benchmark Results}

This appendix contains tables with benchmark results. Tables B.1 and B.2 are discussed in Chapter 6, Table B.3 in Chapter 8, and the remaining tables are discussed in Chapter 11, which also contains plots of the results listed here. All benchmarks were performed on a Pentium 4 running Debian GNU/Linux with a low load.

Table B.1 gives an overview of the results of running a set of benchmarks with and without the guard simplification optimization of Chapter 6 . These benchmarks were performed in SWI-Prolog 5.5.2. The first column indicates the benchmark name and the parameters that were used. The second and third column indicate whether mode and type declarations were provided, respectively. The fourth column indicates whether guard simplification was enabled. In all these columns, an empty cell means the choice has no influence on the resulting compiled code (so it can be "yes" or "no"). The fifth column shows the size of the resulting compiled Prolog code as a pair of the form (\#Clauses ; \#Lines), not including auxiliary predicates. The last column shows the runtime in seconds and a percentage comparing the runtime to that of the version with mode information but without guard simplification. If a cell contains an equality sign ("="), we could not measure any performance difference compared to the version in the row just above that cell. If a cell contains an equivalence sign ("三"), the Prolog code for that row is identical to the one in the row just above. For every benchmark, the results for hand-optimized Prolog code are included, representing the ideal target code.

Table B.2 shows the results of the occurrence subsumption optimization of Chapter 6 for the rules of Example 18 (page 99). The benchmark queries are of the form $\mathrm{a}(1,1,1), \mathrm{a}(2,2,2), \ldots, \mathrm{a}(5000,5000,5000)$; note that none of the rules actually fire so the rule bodies (which are not shown in Example 18) are irrelevant. Symmetry and set semantics analyses were disabled in both cases because they are special cases of occurrence subsumption. The second column in- 


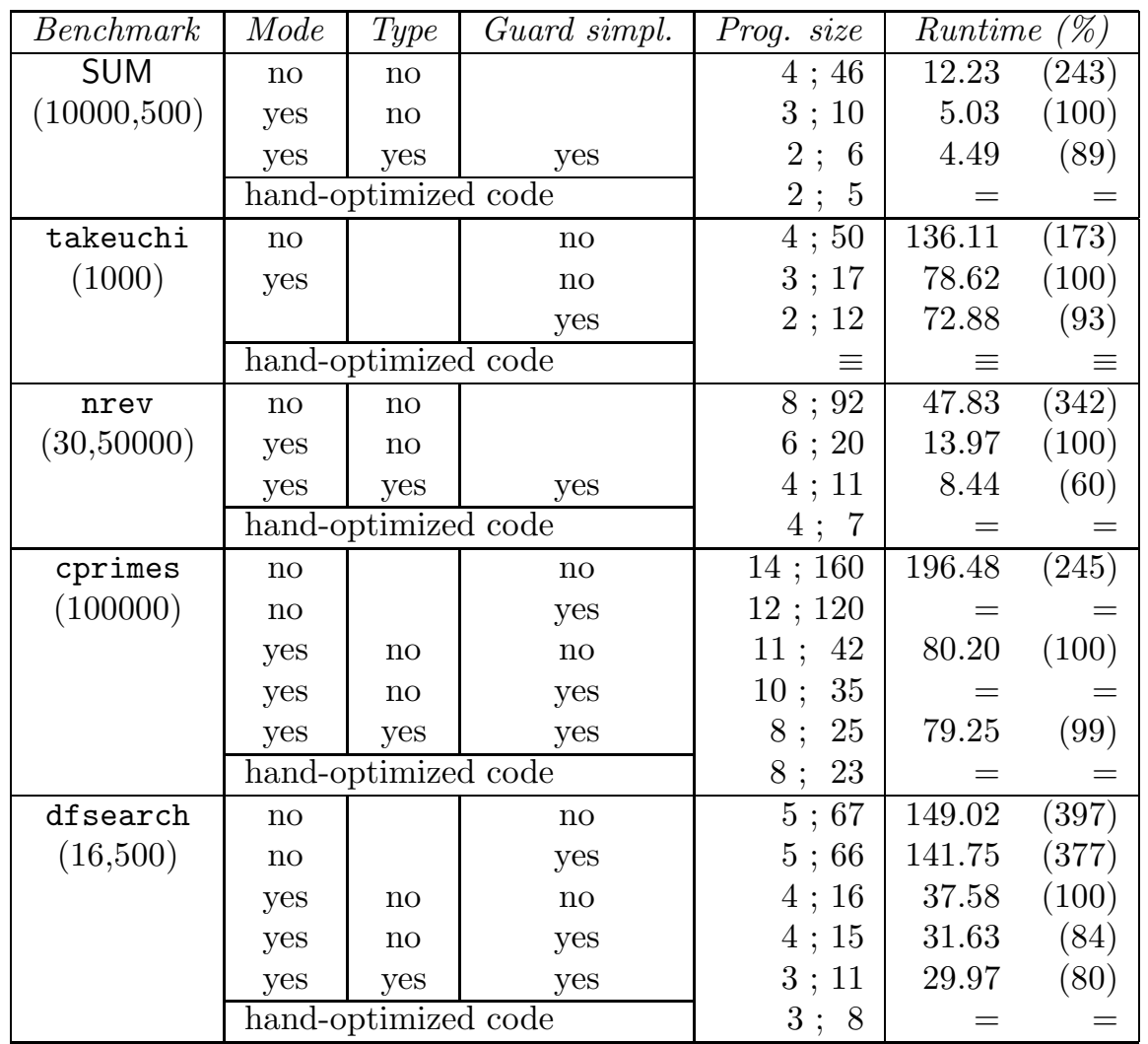

Table B.1: Benchmarks for guard simplification

dicates whether occurrence subsumption was enabled. The third column indicates the number of non-passive occurrences. The runtime column is as in Table B.1.

Table B.3 lists the results for the memory reuse optimizations of Chapter 8 . We used the Leuven CHR system in hProlog 2.4.23. We used a sufficiently large initial heap size so no garbage collection is performed, allowing accurate memory usage statistics. Note that in a realistic setting, a reduction in memory use implies less garbage collection, which results in further speedups. The first column shows the benchmark name ${ }^{1}$ and problem size. Some of the benchmark programs were introduced in Chapter 3 (in particular, in Section 3.5). Columns two, three and four indicate which optimizations were enabled: the abbreviations denote respectively Inlining, Suspension reuse, and In-place updates. The fifth column shows the ratio of suspension cache hits at suspension creation. The next four columns

\footnotetext{
${ }^{1}$ These benchmarks are available at http://www.cs.kuleuven.be/ toms/Research/CHR/.
} 


\begin{tabular}{|c|c|c|cr|}
\hline Benchmark & Occ. subsumption & \# occurrences & \multicolumn{2}{|c|}{ Runtime (\%) } \\
\hline $\mathrm{a}$ & no & 3 & 52.8 & $(100)$ \\
$(5000)$ & yes & 1 & 17.1 & $(32)$ \\
\hline $\mathrm{b}$ & no & 3 & 52.1 & $(100)$ \\
$(5000)$ & yes & 2 & 34.3 & $(66)$ \\
\hline $\mathrm{c}$ & no & 6 & 86.5 & $(100)$ \\
$(5000)$ & yes & 1 & 17.2 & $(20)$ \\
\hline $\mathrm{d}$ & no & 6 & 84.7 & $(100)$ \\
$(5000)$ & yes & 3 & 50.3 & $(59)$ \\
\hline $\mathrm{e}$ & no & 6 & 86.4 & $(100)$ \\
$(5000)$ & yes & 3 & 49.9 & $(58)$ \\
\hline $\mathrm{f}$ & no & 4 & 64.4 & $(100)$ \\
$(5000)$ & yes & 3 & 47.8 & $(74)$ \\
\hline
\end{tabular}

Table B.2: Benchmarks for occurrence subsumption

list the runtime in seconds, the total amount of (heap+trail) memory used (in 32-bit words) and percentages indicating relative time and memory use. The last column contains some comments on case-specific properties of the optimizations: whether the (most important) in-place updated constraint has active occurrences ("active occs") for which additional clauses had to be generated because of interference with late storage ("new clauses"), the number of potentially modified indexed arguments ("index-args"), the number of in-place updates in the generated code ("inpl. upd."), and whether the propagation history ("history reset") and the constraint identifier ("new identifier") suspension fields need to be updated.

The results shown in Table B.5 were obtained as follows. We have used the same version of hProlog for the CHR(Prolog) program as for the Prolog program. For the Haskell program, we have used the Glasgow Haskell Compiler ${ }^{2}$ version 6.6 with option "-02". We have used Maude version 2.2 and Jess version 7.0. Garbage collection complicates accurate measurement of memory usage, which is why no memory results are given. However, the runtimes include time spent in garbage collection. In this way, space complexity is implicitly taken into account. Some benchmarks run out of memory (indicated by "mem") when the maximal heap size is set to a value slightly lower than the amount of physical memory available on the test machine $(512 \mathrm{Mb})$.

Finally, the execution times listed in Tables B.6 and B.7 were obtained as follows. We used gcc version 3.3.5 with the -04 option to compile the C programs, hProlog version 2.4.39-32 with the Leuven CHR compiler by Schrijvers and Demoen (2004), SICStus Prolog version 3.12.2 (x86-linux-glibc2.2) with CHR version 2.2 by Holzbaur and Frühwirth (1998), and JCHR version 1.3.3 by Van

\footnotetext{
${ }^{2}$ GHC home page: http://www.haskell.org/ghc/
} 
Benchmark Results

\begin{tabular}{|c|c|c|c|c|c|c|c|c|c|}
\hline Benchmark & Inl & $S R$ & $I U$ & Hits & Runtime & Memory & \%time & $\%$ mem & \multirow[t]{4}{*}{ Notes } \\
\hline \multirow{3}{*}{$\begin{array}{l}\text { bool_chain } \\
(1000)\end{array}$} & \multirow{3}{*}{$\sqrt{ }$} & \multirow[b]{3}{*}{$\sqrt{ }$} & & \multirow[b]{3}{*}{$\%$} & 20.78 & 1756826 & $233.7 \%$ & 101.6\% & \\
\hline & & & & & 8.89 & 1728326 & $100.0 \%$ & $100.0 \%$ & \\
\hline & & & & & 9.93 & 1726263 & $111.7 \%$ & $99.9 \%$ & \\
\hline \multirow{5}{*}{$\begin{array}{l}\text { FIB-HEAP } \\
\text { (DIJKSTRA) } \\
(65536)\end{array}$} & \multirow{5}{*}{$\begin{array}{l}\sqrt{ } \\
\sqrt{ } \\
\sqrt{ } \\
\sqrt{ }\end{array}$} & \multirow{5}{*}{$\sqrt{ }$} & & \multirow{4}{*}{$78 \%$} & 7.16 & 61992261 & $141.2 \%$ & 198.3\% & \multirow{5}{*}{$\begin{array}{l}\text { active occs } \\
\text { new clauses } \\
3 \text { index-args } \\
\text { (4 inpl. upd.) }\end{array}$} \\
\hline & & & & & 5.07 & 31256958 & $100.0 \%$ & $100.0 \%$ & \\
\hline & & & & & 5.39 & 24064894 & $106.3 \%$ & $77.0 \%$ & \\
\hline & & & $\sqrt{ }$ & & 5.22 & 29730416 & $103.0 \%$ & $95.1 \%$ & \\
\hline & & & $\sqrt{ }$ & $72 \%$ & 5.60 & 24111191 & $110.5 \%$ & $77.1 \%$ & \\
\hline \multirow[t]{5}{*}{ inference } & \multirow[b]{5}{*}{$v$} & \multirow{5}{*}{$\sqrt{ }$} & & & 0.111 & 160057 & $111.0 \%$ & $111.3 \%$ & \multirow{5}{*}{$\begin{array}{l}\text { history reset } \\
\text { new identifier } \\
\text { active occs } \\
25 \text { index-args } \\
\text { (17 inpl. } \text { upd.) }\end{array}$} \\
\hline & & & & & 0.100 & 143831 & $100.0 \%$ & $100.0 \%$ & \\
\hline & & & & & 0.105 & 139958 & $105.0 \%$ & $97.3 \%$ & \\
\hline & & & $\sqrt{ }$ & & 0.100 & 143161 & $100.0 \%$ & $99.5 \%$ & \\
\hline & & & $\sqrt{ }$ & $9 \%$ & 0.104 & 140427 & $104.0 \%$ & $97.6 \%$ & \\
\hline \multirow{3}{*}{$\begin{array}{l}\text { PRIMES } \\
(10000)\end{array}$} & & \multirow[b]{3}{*}{$\sqrt{ }$} & & \multirow[b]{3}{*}{$0 \%$} & 21.23 & 40924601 & $129.5 \%$ & $100.5 \%$ & \\
\hline & & & & & 16.39 & 40734 & $100.0 \%$ & $100.0 \%$ & \\
\hline & & & & & 6.73 & 1613 & $41.1 \%$ & $39.6 \%$ & \\
\hline \multirow{5}{*}{$\begin{array}{l}\text { RAMSIMUL } \\
(1000000)\end{array}$} & & \multirow{5}{*}{$\sqrt{ }$} & & \multirow{4}{*}{$100 \%$} & 12.41 & 88003112 & $149.7 \%$ & $293.3 \%$ & \multirow{5}{*}{$\begin{array}{l}\text { no active occs } \\
\text { no index-args } \\
\text { (8 inpl. upd.) }\end{array}$} \\
\hline & $\sqrt{ }$ & & & & 8.29 & 30002947 & $100.0 \%$ & $100.0 \%$ & \\
\hline & 1 & & & & 6.19 & 6002961 & $74.7 \%$ & $20.0 \%$ & \\
\hline & $\sqrt{ }$ & & $\sqrt{ }$ & & 5.13 & 2961 & $61.9 \%$ & $0.0 \%$ & \\
\hline & $\sqrt{ }$ & & $\sqrt{ }$ & $0 \%$ & 5.23 & 2961 & $63.1 \%$ & $0.0 \%$ & \\
\hline \multirow{5}{*}{\begin{tabular}{|l} 
SUDOKU \\
$(283576$ \\
solutions $)$
\end{tabular}} & \multirow{5}{*}{$\begin{array}{l}\sqrt{ } \\
\sqrt{ } \\
\sqrt{ }\end{array}$} & & & & 30.28 & 112483 & $118.9 \%$ & 121.8\% & \\
\hline & & & & & 109.60 & 92387 & $100.0 \%$ & $100.0 \%$ & no active oc \\
\hline & & $\sqrt{ }$ & & $100 \%$ & 107.42 & 23329 & $98.0 \%$ & $25.3 \%$ & no index-are \\
\hline & & & $\sqrt{ }$ & & 60.89 & 20515 & $55.6 \%$ & $22.2 \%$ & (3 inpl. upd \\
\hline & & $\sqrt{ }$ & $\sqrt{ }$ & $\%$ & 63.65 & 20187 & $58.1 \%$ & $21.9 \%$ & \\
\hline UNIC & & & & & 8.85 & 66611812 & $132.9 \%$ & $154.9 \%$ & new identifie \\
\hline & $\checkmark$ & & & & 6.66 & 43013248 & $100.0 \%$ & $100.0 \%$ & active occs \\
\hline & $\sqrt{ }$ & $\sqrt{ }$ & & $52 \%$ & 5.94 & 32302433 & $89.2 \%$ & $75.1 \%$ & new clauses \\
\hline & $\sqrt{ }$ & & $\sqrt{ }$ & & 5.14 & 29064918 & $77.2 \%$ & $67.6 \%$ & 3 index-args \\
\hline & $\sqrt{ }$ & $\sqrt{ }$ & $\sqrt{ }$ & $0 \%$ & 5.41 & 28664915 & $81.2 \%$ & $66.6 \%$ & (8 inpl. upd \\
\hline ZEBRA & & & & & 0.181 & 41904 & $116.0 \%$ & $131.8 \%$ & \\
\hline & 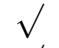 & & & & 0.156 & 31798 & $100.0 \%$ & $100.0 \%$ & active occs \\
\hline & 1 & $\sqrt{ }$ & & $97 \%$ & 0.148 & 26407 & $94.9 \%$ & $83.0 \%$ & no index-ar \\
\hline & ( & & $\sqrt{ }$ & & 0.131 & 24915 & $84.0 \%$ & $78.4 \%$ & (2 inpl. upd \\
\hline & $\sqrt{ }$ & $\sqrt{ }$ & $\sqrt{ }$ & $38 \%$ & 0.136 & 24625 & $87.2 \%$ & $77.4 \%$ & \\
\hline
\end{tabular}

Table B.3: Benchmarks for suspension reuse and in-place updates 
(a) Loop

$i$ (1, add , 1, 3)

i (2, sub , 1, 2)

$i(3$, c jmp , 2, 5)

$i(4, j m p, 1)$

$i(5$, halt $)$

$\mathrm{m}(1,1)$

$\mathrm{m}(2, n)$

$\mathrm{m}(3,0)$

$c(1)$ (b) MFib

$$
\begin{array}{ll}
i(1, \text { init }, 3) & \\
i(2, i \mathrm{mv}, 1,6) & \\
i(3, \mathrm{~mm}, 2,7) & \mathrm{m}(1,8) \\
i(4, \mathrm{mul}, 6,7) & \mathrm{m}(2,9) \\
i(5, \mathrm{mvi}, 7,3) & \mathrm{m}(3,10) \\
i(6, \text { add }, 5,1) & \mathrm{m}(4, n) \\
i(7, \text { add }, 5,2) & \mathrm{m}(5,1) \\
i(8, \text { add }, 5,3) & \mathrm{m}(6,0) \\
i(9, \text { sub }, 5,4) & \mathrm{m}(7,0) \\
i(10, \mathrm{cjmp}, 4,12) & \mathrm{m}(8,1) \\
i(11, \text { jmp }, 1) & \mathrm{m}(9,1) \\
i(12, \text { halt }) & \mathrm{c}(1)
\end{array}
$$

\begin{tabular}{|c|c|c|c|c|c|c|c|c|c|}
\hline \multirow{2}{*}{$\begin{array}{c}\text { Query } \\
n\end{array}$} & \multicolumn{3}{|c|}{ CHR(Prolog) } & \multirow[t]{2}{*}{ Prolog } & \multicolumn{2}{|c|}{ Haskell } & \multicolumn{2}{|c|}{ Maude } & \multirow[t]{2}{*}{ Jess } \\
\hline & 2 & 1 & 0 & & lazy & strict & naive & Map & \\
\hline Loop & & & & & & & & & \\
\hline $10^{\wedge} 4$ & 0.09 & 0.32 & 0.32 & 0.05 & 0.05 & 0.02 & 1.01 & 0.37 & 3.48 \\
\hline $10^{\wedge} 5$ & 0.88 & 3.23 & 3.10 & 0.44 & 0.41 & 0.21 & 10.09 & 3.48 & 24.07 \\
\hline $10^{\wedge} 6$ & 8.70 & 32.42 & 29.93 & 4.46 & 5.40 & 2.05 & 100.43 & 35.32 & 231.13 \\
\hline $10^{\wedge} 7$ & 86.95 & 324.91 & 298.11 & 42.88 & mem & 20.41 & 987.28 & 371.54 & 2266.33 \\
\hline MFib & & & & & & & & & \\
\hline $10^{\wedge} 3$ & 0.03 & 0.07 & 2.20 & 0.05 & 0.04 & 0.02 & 32.00 & 0.24 & 3.08 \\
\hline $10^{\wedge} 4$ & 0.26 & 0.69 & 434.09 & 0.60 & 1.19 & 0.16 & 4772.70 & 3.69 & 10.22 \\
\hline $10^{\wedge} 5$ & 2.61 & 7.39 & 一 & 7.40 & mem & 1.82 & - & 52.12 & 98.72 \\
\hline $10^{\wedge} 6$ & 26.76 & 76.43 & & 89.37 & & 19.69 & & 1650.49 & mem \\
\hline NLoop & & & & & & & & & \\
\hline $2^{\wedge} 7$ & 0.18 & 0.44 & 4.02 & 0.26 & 0.17 & 0.08 & 57.05 & 1.08 & 4.75 \\
\hline $2 \wedge 8$ & 0.71 & 1.72 & 26.81 & 1.10 & 0.73 & 0.34 & 419.54 & 4.68 & 19.59 \\
\hline $2^{\wedge} 9$ & 2.79 & 6.86 & 192.55 & 4.83 & 9.43 & 1.16 & 3677.66 & 21.51 & 77.81 \\
\hline $2 \wedge 10$ & 11.16 & 27.57 & 1475.77 & 20.49 & mem & 4.82 & - & 111.13 & 295.20 \\
\hline $2^{\wedge} 11$ & 44.28 & 108.87 & 11620.42 & 90.04 & & 20.37 & & 530.60 & 1258.71 \\
\hline $2^{\wedge} 12$ & 178.22 & 434.48 & - & 380.78 & & 85.27 & & 2604.68 & 4997.12 \\
\hline $2^{\wedge} 13$ & 709.89 & 1744.55 & & 1678.73 & & 356.29 & & 1474.24 & 20147.51 \\
\hline $2^{\wedge} 14$ & 2864.56 & 7050.31 & & 7272.05 & & 500.36 & & 1573.71 & \\
\hline
\end{tabular}

(c) NLoop

$$
\begin{aligned}
& i(1, \operatorname{mov}, 2,3) \quad i(16, \operatorname{mov}, 3,5) \\
& i(2 \text {, add }, 1,3) \quad i(17, \text { sub }, 1,5) \\
& i(3, \text { init, } 3) \quad i(18, \text { cjmp }, 5,20) \\
& i(4, \mathrm{mvi}, 4,3) \quad i(19, \mathrm{jmp}, 12) \\
& i(5, \text { sub , 4, 3) } \quad i(20, \text { sub , 4 , 2) } \\
& i(6, \text { mov }, 3,5) \quad i(21, \text { cjmp , 2, 23) } \\
& i(7, \text { sub }, 1,5) \quad i(22, \text { jmp , 10) } \\
& i(8, \text { cjmp , 5, 10) } i(23 \text {, halt }) \\
& i(9, \text { jmp }, 3) \\
& i(10, \operatorname{mov}, 2,3) \mathrm{m}(1,10) \\
& \mathrm{i}(11, \text { add }, 1,3) \mathrm{m}(2, n) \\
& \mathrm{i}(12, \mathrm{imv}, 3,5) \mathrm{m}(3,0) \\
& i(13 \text {, add }, 4,5) \quad m(4,1) \\
& \mathrm{i}(14, \mathrm{mvi}, 5,3) \mathrm{m}(5,0) \\
& i(15, \text { sub , 4, 3) } c(1)
\end{aligned}
$$

Table B.4: Example RAM simulator queries used for benchmarking

Table B.5: Benchmarks for several RAM simulator implementations 


\begin{tabular}{|c|c|ccc|c|c|}
\hline & C & \multicolumn{3}{|c|}{ CHR(hProlog) } & CHR(SICStus) & CHR(Java) \\
$n$ & 2 & 2 & 1 & 0 & 0 & 2 \\
\hline $1 \mathrm{k}$ & $<0.01$ & 0.01 & 0.02 & 1.46 & 2.14 & 0.01 \\
$2 \mathrm{k}$ & $<0.01$ & 0.02 & 0.03 & 7.62 & 8.84 & 0.01 \\
$4 \mathrm{k}$ & $<0.01$ & 0.03 & 0.07 & 32.94 & 33.12 & 0.03 \\
$8 \mathrm{k}$ & $<0.01$ & 0.06 & 0.14 & 112.19 & 119.21 & 0.07 \\
$16 \mathrm{k}$ & $<0.01$ & 0.12 & 0.27 & 367.93 & 465.33 & 0.14 \\
$32 \mathrm{k}$ & 0.01 & 0.26 & 0.56 & 1245.44 & $>1000$ & 0.47 \\
$64 \mathrm{k}$ & 0.02 & 0.53 & 1.17 & $>1000$ & & 1.36 \\
$128 \mathrm{k}$ & 0.09 & 1.18 & 2.33 & & & 3.58 \\
$256 \mathrm{k}$ & 0.24 & 2.31 & 5.03 & & & 7.70 \\
$512 \mathrm{k}$ & 0.53 & 4.80 & 10.37 & & & 18.59 \\
$1 \mathrm{~m}$ & 1.12 & 9.91 & 21.60 & & & 64.82 \\
\hline
\end{tabular}

Table B.6: Benchmarks for the union-find algorithm, in different systems

\begin{tabular}{|c|c|ccc|c|c|}
\hline & C & \multicolumn{3}{|c|}{ CHR(hProlog) } & CHR(SICStus) & CHR(Java) \\
$n$ & 2 & 2 & 1 & 0 & 0 & 2 \\
\hline $1 \mathrm{k}$ & $<0.01$ & 0.05 & 0.13 & 3.47 & 6.42 & 0.17 \\
$4 \mathrm{k}$ & 0.01 & 0.26 & 0.57 & 66.24 & 96.89 & 0.65 \\
$16 \mathrm{k}$ & 0.07 & 1.07 & 2.33 & 717.92 & $>1000$ & 3.77 \\
$64 \mathrm{k}$ & 0.42 & 4.56 & 9.85 & $>1000$ & & stack overflow \\
$128 \mathrm{k}$ & 0.94 & 9.53 & 20.18 & & & \\
$256 \mathrm{k}$ & 2.06 & 19.85 & 41.12 & & & \\
\hline
\end{tabular}

Table B.7: Benchmarks for Dijkstra's algorithm, in different systems

Weert et al. (2005), executed in Java 1.5.0_03 with the "server" virtual machine and background compilation disabled (options - server -Xbatch). For the C programs, we listed the user cpu time. For the CHR programs in Prolog systems, we measured user cpu time, not including garbage collection time. For the JCHR programs, we measured clock time. We made sure the test machine had a very low load, the Java heap size was set as big as possible while still fitting in RAM, and we took the best time of five runs. We tested on a Pentium 4 machine with $512 \mathrm{MB}$ of RAM and a cpu with a clock speed of $1.7 \mathrm{GHz}$ (cache size $256 \mathrm{~KB}$ ), running Debian GNU/Linux version 3.1 (sarge) with Linux kernel 2.6.15. 


\section{Bibliography}

In the following list of references, we have used the usual acronyms for common workshop, conference, book series and journal names:

CHR - Workshop on Constraint Handling Rules

$\mathrm{CP}$ - International Conference on Principles and Practice of Constraint Programming

ENTCS - Electronic Notes in Theoretical Computer Science (Elsevier)

ICLP - International Conference on Logic Programming

LNCS - Lecture Notes in Computer Science (Springer)

LNAI - Lecture Notes in Artificial Intelligence (Springer)

LOPSTR - Intl. Symposium on Logic-Based Program Synthesis and Transformation

PPDP - Intl. ACM SIGPLAN Conf. Principles 8 Practice of Declarative Programming

TPLP - Theory and Practice of Logic Programming (Cambridge University Press)

WLPE - Workshop on Logic Programming Environments

AbDennadHeR, S. 2000. A language for experimenting with declarative paradigms. In RCoRP'00(bis): Proc. 2nd Workshop on Rule-Based Constraint Reasoning and Programming, Singapore, T. Frühwirth et al., Eds.

Abdennadher, S. 2001. Rule-based constraint programming: Theory and practice. Habilitationsschrift. Inst. of Comp. Sc., LMU, Munich, Germany.

Abdennadher, S. And Christiansen, H. 2000. An experimental CLP platform for integrity constraints and abduction. In FQAS'00: Proc. 4th Intl. Conf. Flexible Query Answering Systems, Warsaw, Poland, pp. 141-152.

Abdennadher, S. And Frühwirth, T. 1998. On completion of Constraint Handling Rules. In $C P^{\prime} 98$, Pisa, Italy, pp. 25-39, M. J. Maher and J.-F. Puget, Eds. LNCS, vol. 1520.

Abdennadher, S. And Frühwirth, T. 1999. Operational equivalence of CHR programs and constraints. In $C P^{\prime} 99$, Alexandria, VA, USA, pp. 43-57, J. Jaffar, Ed. LNCS, vol. 1713.

Abdennadher, S., Frühwirth, T., And Holzbaur, C., Eds. 2005. Special Issue on Constraint Handling Rules. TPLP, vol. 5(4-5). 
Abdennadher, S., Frühwirth, T., And Meuss, H. 1999. Confluence and semantics of constraint simplification rules. Constraints 4, 2, 133-165.

Abdennadher, S., Krämer, E., Saft, M., And Schmauss, M. 2002. JACK: A Java Constraint Kit. In WFLP'01: Proc. 10th Intl. Workshop on Functional and (Constraint) Logic Programming, Selected Papers, Kiel, Germany, pp. 1-17, M. Hanus, Ed. ENTCS, vol. 64.

Abdennadher, S. AND MARTe, M. 2000. University course timetabling using Constraint Handling Rules. In J. AAI, Holzbaur and Frühwirth (2000b), pp. 311325 .

Abdennadher, S. And Rigotti, C. 2004. Automatic generation of rule-based constraint solvers over finite domains. ACM TOCL 5, 2, 177-205.

Abdennadher, S. And Rigotti, C. 2005. Automatic generation of CHR constraint solvers. In TPLP, Abdennadher et al. (2005), pp. 403-418.

Abdennadher, S., SAFt, M., AND Will, S. 2000. Classroom assignment using constraint logic programming. In PACLP'00: Proc. 2nd Intl. Conf. and Exhibition on Practical Application of Constraint Technologies and Logic Programming, Manchester, UK.

AbDennadher, S. And Schütz, H. 1998. CHR ${ }^{\vee}$, a flexible query language. In FQAS'98, Roskilde, Denmark, pp. 1-14, T. Andreasen, H. Christiansen, and H. Larsen, Eds. LNAI, vol. 1495.

Abdennadher, S. And SobHi, I. 2008. Generation of rule-based constraint solvers: Combined approach. In LOPSTR'0\%, Kongens Lyngby, Denmark, A. King, Ed. LNCS, vol. 4915.

Adelson-Velsky, G. M. And Landis, E. M. 1962. An algorithm for the organization of information. Doklady Akademii Nauk SSSR 146, 263-266.

Agrawal, M., Kayal, N., And Saxena, N. 2004. PRIMES is in P. Annals of Mathematics 160, 2, 781-793.

Aho, A. V., Hopcroft, J. E., And Ullman, J. D. 1975. The Design and Analysis of Computer Algorithms. Addison-Wesley Longman.

Alberti, M., Daolio, D., Torroni, P., Gavanelli, M., Lamma, E., And MELlO, P. 2004. Specification and verification of agent interaction protocols in a logic-based system. In SAC'04: Proc. 19th ACM Symp. Applied Computing, Nicosia, Cyprus, pp. 72-78, H. Haddad et al., Eds. ACM Press.

Alberti, M., Gavanelli, M., Lamma, E., Chesani, F., Mello, P., and TorRONI, P. 2006. Compliance verification of agent interaction: a logic-based software tool. Applied Artificial Intelligence 20, 2-4, 133-157.

Alberti, M., Gavanelli, M., Lamma, E., Mello, P., and Torroni, P. 2004. Specification and verification of agent interaction using social integrity con- 
straints. In LCMAS'03: Logic and Communication in Multi-Agent Systems, Eindhoven, the Netherlands, pp. 94-116. ENTCS, vol. 85(2).

Apt, K. R. And Monfroy, E. 2001. Constraint programming viewed as rulebased programming. TPLP 1, 6, 713-750.

Aurélio, M., Fages, F., And Robin, J. 2008. Default reasoning in $\mathrm{CHR}^{\vee}$. In CHR'08, Schrijvers et al. (2008), pp. 111-125.

Badea, L., Tilivea, D., And Hotaran, A. 2004. Semantic Web Reasoning for Ontology-Based Integration of Resources. In PPSWR'04: Proc. 2nd Intl. Workshop on Principles And Practice Of Semantic Web Reasoning, Saint-Malo, France, pp. 61-75. LNCS, vol. 3208.

BArRAnCO-MEndozA, A. 2005. Stochastic and heuristic modelling for analysis of the growth of pre-invasive lesions and for a multidisciplinary approach to early cancer diagnosis. Ph.D. thesis, Simon Fraser University, Burnaby, Canada.

BAvarian, M. AND DAHL, V. 2006. Constraint based methods for biological sequence analysis. J. Universal Computer Science 12, 11, 1500-1520.

Bayardo, JR., R. J. And Miranker, D. P. 1996. Processing queries for firstfew answers. In CIKM'96: Proc. 5th Intl. Conf. Information and Knowledge Management, Rockville, MD, USA, pp. 45-52.

Betz, H. 2007. Relating coloured Petri nets to Constraint Handling Rules. In CHR'07, Djelloul et al. (2007), pp. 33-47.

Betz, H. ANd Frühwirth, T. 2005. A linear-logic semantics for Constraint Handling Rules. In CP'05, Sitges, Spain, pp. 137-151, P. van Beek, Ed. LNCS, vol. 3709.

Betz, H. And Frühwirth, T. 2007. A linear-logic semantics for Constraint Handling Rules with disjunction. In CHR'0\%, Djelloul et al. (2007), pp. 17-31.

Bistarelli, S., Frühwirth, T., Marte, M., and Rossi, F. 2004. Soft constraint propagation and solving in Constraint Handling Rules. Computational Intelligence: Special Issue on Preferences in AI and CP 20, 2 (May), 287-307.

Boespflug, M. 2007. TaiChi:how to check your types with serenity. The Monad.Reader 9, 17-31.

Bouissou, O. 2004. A CHR library for SiLCC. Diplomathesis. Tech. Univ. Berlin, Germany.

BRAND, S. 2002. A note on redundant rules in rule-based constraint programming. In CSCLP'02: Joint ERCIM/CologNet Intl. Workshop on Constraint Solving and Constraint Logic Programming, Selected papers, Cork, Ireland, pp. 279-336. LNCS, vol. 2627.

Brand, S. And Monfroy, E. 2003. Deductive generation of constraint propagation rules. In RULE'03: 4th Intl. Workshop on Rule-Based Programming, Valencia, Spain, pp. 45-60, G. Vidal, Ed. ENTCS, vol. 86(2). 
Bratko, I. 2001. Prolog Programming for Artificial Intelligence, Third Edition. Addison-Wesley/Pearson Education.

Bressan, S. And GOH, C. H. 1998. Answering queries in context. In FQAS'98, Roskilde, Denmark, pp. 68-82, T. Andreasen, H. Christiansen, and H. Larsen, Eds. LNAI, vol. 1495.

Brodal, G. S. AND OKASAKI, C. 1996. Optimal purely functional priority queues. J. Functional Programming 6, 6, 839-857.

Cabedo, L. M. And Escrig, M. T. 2003. Modeling motion by the integration of topology and time. J. Universal Computer Science 9, 9, 1096-1122.

Cherkassky, B. V., Goldberg, A. V., And Radzik, T. 1996. Shortest paths algorithms: Theory and experimental evaluation. Mathematical Programming 73, 129-174.

Chin, W.-N., Sulzmann, M., And Wang, M. 2003. A type-safe embedding of Constraint Handling Rules into Haskell. Honors Thesis. School of Computing, National University of Singapore.

Christiansen, H. 2005a. CHR grammars. In TPLP, Abdennadher et al. (2005), pp. $467-501$.

Christiansen, H. 2005b. Reasoning about passive declarations in chr. In CHR'05, Schrijvers and Frühwirth (2005b), pp. 93-108.

Christiansen, H. 2006. On the implementation of global abduction. In CLIMA'06: 7th Intl. Workshop on Computational Logic in Multi-Agent Systems - Revised, Selected and Invited Papers, Hakodate, Japan, pp. 226-245, K. Inoue, K. Satoh, and F. Toni, Eds. LNCS, vol. 4371.

Christiansen, H. And Dahl, V. 2005. HYPROLOG: A new logic programming language with assumptions and abduction. In ICLP'05, Sitges, Spain, pp. 159173, M. Gabbrielli and G. Gupta, Eds. LNCS, vol. 3668.

Clavel, M., Durán, F., Eker, S., Lincoln, P., et Al. 2002. Maude: Specification and programming in rewriting logic. Theoretical Computer Science 285, 2 , 187-243.

Clocksin, W. F. And Mellish, C. S. 1984. Programming in Prolog. Springer.

Cormen, T. H., Leiserson, C. E., Rivest, R. L., And Stein, C. 2001. Introduction to Algorithms, Second Edition. MIT Press and McGraw-Hill.

Crane, C. A. 1972. Linear lists and priority queues as balanced binary trees. Ph.D. thesis, Stanford University, CA, USA.

DAHL, V. AND Blache, P. 2005. Extracting selected phrases through constraint satisfaction. In Proc. 2nd Intl. Workshop on Constraint Solving and Language Processing, Sitges, Spain. 
DAHL, V. AND GU, B. 2006. Semantic property grammars for knowledge extraction from biomedical text. In ICLP'06, Seattle, Washington, pp. 442-443, S. Etalle and M. Truszczynski, Eds. LNCS, vol. 4079.

DAhL, V. AND VolL, K. 2004. Concept formation rules: An executable cognitive model of knowledge construction. In NLUCS'04: Proc. First Intl. Workshop on Natural Language Understanding and Cognitive Sciences, Porto, Portugal.

De Koninck, L., Schrijvers, T., And Demoen, B. 2006a. INCLP(R) - Intervalbased nonlinear constraint logic programming over the reals. In WLP'06, Fink et al. (2006), pp. 91-100.

De Koninck, L., Schrijvers, T., And Demoen, B. 2006b. Search strategies in CHR(Prolog). In CHR'06, Schrijvers and Frühwirth (2006), pp. 109-123.

De Koninck, L., Schrijvers, T., And Demoen, B. 2007a. The correspondence between the Logical Algorithms language and CHR. In ICLP'0'\%, Porto, Portugal, pp. 209-223, V. Dahl and I. Niemelä, Eds. LNCS, vol. 4670.

De Koninck, L., Schrijvers, T., And Demoen, B. 2007b. User-definable rule priorities for CHR. In PPDP'07, Wrocław, Poland, pp. 25-36, M. Leuschel and A. Podelski, Eds.

De Koninck, L. And Sneyers, J. 2007. Join ordering for Constraint Handling Rules. In CHR'07, Djelloul et al. (2007), pp. 107-121.

De Koninck, L., Stuckey, P. J., And Duck, G. J. 2008. Optimizing compilation of CHR with rule priorities. In FLOPS'08: Proc. 9th Intl. Symp. Functional and Logic Programming, Ise, Japan, pp. 32-47, J. Garrigue and M. Hermenegildo, Eds. LNCS, vol. 4989.

Demoen, B. 2002. Dynamic attributes, their hProlog implementation, and a first evaluation. Tech. rep. CW350, K.U.Leuven, Dept. Comp. Sc.

Di Giusto, C., Gabbrielli, M., And Meo, M. C. 2008. Expressiveness of multiple heads in CHR. CoRR abs/0804.3351.

DiJkstra, E. W. 1959. A note on two problems in connexion with graphs. Numerische Mathematik 1, 4, 269-271.

Djelloul, K., DAO, T.-B.-H., And Frühwirth, T. 2007. Toward a first-order extension of Prolog's unification using CHR: a CHR first-order constraint solver over finite or infinite trees. In SAC'07: Proc. 2007 ACM Symp. Applied computing, Seoul, Korea, pp. 58-64. ACM Press.

Djelloul, K., Duck, G. J., And Sulzmann, M., Eds. 2007. CHR'0r: Proc. 4 th Workshop on Constraint Handling Rules, Porto, Portugal.

DuCK, G. J. 2005. Compilation of Constraint Handling Rules. Ph.D. thesis, University of Melbourne, Australia. 
Duck, G. J. And SchriJvers, T. 2005. Accurate functional dependency analysis for Constraint Handling Rules. In CHR'05, Schrijvers and Frühwirth (2005b), pp. 109-124.

Duck, G. J., Stuckey, P. J., And Brand, S. 2006. ACD term rewriting. In ICLP'06, Seattle, Washington, pp. 117-131, S. Etalle and M. Truszczynski, Eds. LNCS, vol. 4079.

Duck, G. J., Stuckey, P. J., García de la Banda, M., and Holzbaur, C. 2003. Extending arbitrary solvers with Constraint Handling Rules. In PPDP'O3, Uppsala, Sweden, pp. 79-90. ACM Press.

Duck, G. J., Stuckey, P. J., García de la Banda, M., And Holzbaur, C. 2004. The refined operational semantics of Constraint Handling Rules. In ICLP'04, Saint-Malo, France, pp. 90-104, B. Demoen and V. Lifschitz, Eds. LNCS, vol. 3132.

Duck, G. J., Stuckey, P. J., And Sulzmann, M. 2007. Observable confluence for Constraint Handling Rules. In ICLP'O7, Porto, Portugal, pp. 224-239, V. Dahl and I. Niemelä, Eds. LNCS, vol. 4670.

EkeR, S. 2003. Associative-commutative rewriting on large terms. In RTA'03: Rewriting Techniques and Applications, Valencia, Spain, pp. 14-29, R. Nieuwenhuis, Ed. LNCS, vol. 2706.

Escrig, M. T. And Toledo, F. 1998a. A framework based on CLP extended with CHRs for reasoning with qualitative orientation and positional information. $J$. Visual Languages and Computing 9, 1, 81-101.

Escrig, M. T. And Toledo, F. 1998b. Qualitative Spatial Reasoning: Theory and Practice - Application to Robot Navigation. IOS Press.

Fages, F., Mario de Oliveira Rodrigues, C., and Martinez, T. 2008. Modular CHR with ask and tell. In CHR'08, Schrijvers et al. (2008), pp. 95-109.

Fink, M., Tompits, H., And Woltran, S., Eds. 2006. WLP'06: Proc. 20th Workshop on Logic Programming, Vienna, Austria. T.U.Wien INFSYS research report 1843-06-02.

FIRAT, A. 2003. Information integration using contextual knowledge and ontology merging. Ph.D. thesis, MIT Sloan School of Management, MA, USA.

Forgy, C. L. 1982. Rete: A fast algorithm for the many pattern / many object pattern match problem. Artificial Intelligence 19, 1, 17-37.

Fredman, M., Sedgewick, R., Sleator, D., And Tarjan, R. 1986. The pairing heap: A new form of self-adjusting heap. Algorithmica 1, 1, 111-129.

Fredman, M. AND TARJAn, R. 1987. Fibonacci heaps and their uses in improved network optimization algorithms. J. ACM 34, 3, 596-615.

Friedman-Hill, E. 2003. Jess in Action: Java Rule-Based Systems. Manning. 
FRÜHwiRTH, T. 1992. Constraint simplification rules. Tech. rep. ECRC-92-18, European Computer-Industry Research Centre, Munich, Germany.

FrüHwIRTh, T. 1998. Theory and practice of Constraint Handling Rules. J. Logic Programming 37, 1-3, 95-138.

Frühwirth, T. 2000. Proving termination of constraint solver programs. In New Trends in Contraints, Paphos, Cyprus, pp. 298-317, K. Apt, A. Kakas, E. Monfroy, and F. Rossi, Eds. LNCS, vol. 1865.

FrüHWIRTH, T. 2001. On the number of rule applications in constraint programs. In Declarative Programming - Selected Papers from AGP 2000, La Habana, Cuba, pp. 147-166, A. Dovier, M. C. Meo, and A. Omicini, Eds. ENTCS, vol. 48.

Frühwirth, T. 2002a. As time goes by: Automatic complexity analysis of simplification rules. In KR'02: Proc. 8th Intl. Conf. Princ. Knowledge Representation and Reasoning, Toulouse, France, pp. 547-557, D. Fensel, F. Giunchiglia, D. McGuinness, and M.-A. Williams, Eds. Morgan Kaufmann.

FrüHwirTh, T. 2002b. As time goes by II: More automatic complexity analysis of concurrent rule programs. In QAPL'01: Proc. First Intl. Workshop on Quantitative Aspects of Programming Languages, Florence, Italy, A. D. Pierro and H. Wiklicky, Eds. ENTCS, vol. 59(3).

FrüHWIRTH, T. 2005a. Logical rules for a lexicographic order constraint solver. In CHR'05, Schrijvers and Frühwirth (2005b), pp. 79-91.

Frühwirth, T. 2005b. Parallelizing union-find in Constraint Handling Rules using confluence. In ICLP'05, Sitges, Spain, pp. 113-127, M. Gabbrielli and G. Gupta, Eds. LNCS, vol. 3668.

Frühwirth, T. 2006a. Complete propagation rules for lexicographic order constraints over arbitrary domains. In CSCLP'05: Recent Advances in Constraints, Joint ERCIM/CoLogNET Intl. Workshop on Constraint Solving and CLP, Revised Selected and Invited Papers, Uppsala, Sweden, B. Hnich, M. Carlsson, F. Fages, and F. Rossi, Eds. LNAI, vol. 3978.

FrüHwIRTh, T. 2006b. Deriving linear-time algorithms from union-find in CHR. In CHR'06, Schrijvers and Frühwirth (2006), pp. 49-60.

FrüHWIRTh, T. 2007. Description logic and rules the CHR way. In CHR'Or, Djelloul et al. (2007), pp. 49-61.

FrüHwirth, T. 2009. Constraint Handling Rules. Cambridge University Press.

Frühwirth, T. And Abdennadher, S. 2003. Essentials of Constraint Programming. Springer.

Frühwirth, T. AND Brisset, P. 1995. High-level implementations of Constraint Handling Rules. Tech. rep. ECRC-95-20, European Computer-Industry Research Centre, Munich, Germany. 
Frühwirth, T., Di Pierro, A., And Wiklicky, H. 2002. Probabilistic Constraint Handling Rules. In WFLP'02: Proc. 11th Intl. Workshop on Functional and (Constraint) Logic Programming, Selected Papers, Grado, Italy, M. Comini and M. Falaschi, Eds. ENTCS, vol. 76.

Frühwirth, T., Herold, A., Küchenhoff, V., Le Provost, T., Et Al. 1992. Constraint logic programming - an informal introduction. In Logic Programming in Action. LNCS, vol. 636.

Frühwirth, T. AND HolzBAur, C. 2003. Source-to-source transformation for a class of expressive rules. In AGP'03: Joint Conf. Declarative Programming, Reggio Calabria, Italy, pp. 386-397, F. Buccafurri, Ed.

Ganzinger, H. And McAllester, D. A. 2001. A new meta-complexity theorem for bottom-up logic programs. In IJCAR'01: Proc. 1st Intl. Joint Conf. Automated Reasoning, Siena, Italy, pp. 514-528, R. Goré, A. Leitsch, and T. Nipkow, Eds. LNCS, vol. 2083.

Ganzinger, H. And MCAllester, D. A. 2002. Logical algorithms. In ICLP'02, Copenhagen, Denmark, pp. 209-223, P. J. Stuckey, Ed. LNCS, vol. 2401.

Giarratano, J. C. And Riley, G. 1994. Expert Systems: Principles and Programming. PWS Publishing Co.

Gouraud, S.-D. And Gotlieb, A. 2006. Using CHRs to generate functional test cases for the Java card virtual machine. In PADL'06: Proc. 8th Intl. Symp. Practical Aspects of Declarative Languages, Charleston, SC, USA, pp. 1-15, P. Van Hentenryck, Ed. LNCS, vol. 3819.

Hanus, M. 2006. Adding Constraint Handling Rules to Curry. In WLP'06, Fink et al. (2006), pp. 81-90.

Hart, P. E., Nilsson, N. J., And Raphael, B. 1968. A formal basis for the heuristic determination of minimum cost paths. IEEE Transactions on Systems Science and Cybernetics 4, 2, 100-107.

Hartmanis, J. 1971. Computational complexity of random access stored program machines. Theory of Computing Systems 5, 3, 232-245.

Hartmanis, J. And Stearns, R. E. 1965. On the computational complexity of algorithms. Trans. American Mathematical Society 117, 285-306.

Henderson, F., Somogyi, Z., And Conway, T. 1996. Determinism analysis in the Mercury compiler. In Proc. 19th Australian Computer Science Conference, Melbourne, Australia, pp. 337-346.

Holzbaur, C. 1992. Metastructures vs. attributed variables in the context of extensible unification. In PLILP'92: Proc. 4th Intl. Symp. Programming Language Implementation and Logic Programming, Leuven, Belgium, pp. 260-268. LNCS, vol. 631 . 
Holzbaur, C. And FrüHwirth, T. 1998. Constraint Handling Rules reference manual, release 2.2. Tech. rep. TR-98-01, Österreichisches Forschungsinstitut für Artificial Intelligence, Vienna, Austria.

Holzbaur, C. And Frühwirth, T. 1999. Compiling Constraint Handling Rules into Prolog with attributed variables. In PPDP'99, Paris, France, pp. 117-133, G. Nadathur, Ed. LNCS, vol. 1702.

Holzbaur, C. And Frühwirth, T. 2000a. A Prolog Constraint Handling Rules compiler and runtime system. In $J$. AAI, Holzbaur and Frühwirth (2000b), pp. 369-388.

Holzbaur, C. And Frühwirth, T., Eds. 2000b. Special Issue on Constraint Handling Rules. J. Applied Artificial Intelligence, vol. 14(4).

Holzbaur, C., García de la Banda, M., Jeffery, D., and Stuckey, P. 2001. Optimizing compilation of Constraint Handling Rules. In ICLP'01, Paphos, Cyprus, pp. 74-89, P. Codognet, Ed. LNCS, vol. 2237.

Holzbaur, C., García de la Banda, M., Stuckey, P. J., And Duck, G. J. 2005. Optimizing compilation of Constraint Handling Rules in HAL. In TPLP, Abdennadher et al. (2005), pp. 503-531.

Hopcroft, J. E. 1971. An $n \log n$ algorithm for minimizing states in a finite automaton. Tech. rep. STAN-CS-71-190, Stanford University, CA, USA.

Hopcroft, J. E., Motwani, R., And Ullman, J. D. 2001. Introduction to Automata Theory, Languages, and Computation. Addison-Wesley Longman.

Hudak, P., Hughes, J., Jones, S. P., And Wadler, P. 2007. A history of Haskell: Being lazy with class. In HOPL-III: Proc. 3rd ACM SIGPLAN Conf. History of Programming Languages, San Diego, CA, USA, pp. 1-55.

IBARAKI, T. AND KAMEDA, T. 1984. On the optimal nesting order for computing $n$-relational joins. ACM Transactions on Database Systems 9, 3, 482-502.

Jaffar, J. And LAssez, J.-L. 1987. Constraint Logic Programming. In POPL'87: Proc. 14th Annual ACM Symp. Principles of Programming Languages, Munich, Germany, pp. 111-119. ACM Press.

Jaffar, J. And Maher, M. J. 1994. Constraint logic programming: A survey. J. Logic Programming 19/20, 503-581.

Jaffar, J., Maher, M. J., Marriot, K., And Stuckey, P. J. 1998. The semantics of constraint logic programs. J. Logic Programming 37, 1-3, 1-46.

JAntzen, M. AND VALK, R. 1979. Formal properties of place/transition nets. In Proc. Advanced Course on General Net Theory of Processes and Systems, Hamburg, Germany. LNCS, vol. 84.

KAeser, M. AND Meister, M. 2006. Implementation of an F-Logic kernel in CHR. In CHR'06, Schrijvers and Frühwirth (2006), pp. 33-47. 
KIng, D. J. 1994. Functional binomial queues. In Proc. Glasgow Workshop on Functional Programming, Ayr, Scotland.

Knuth, D. E. 1997-1998. The Art of Computer Programming, Volumes 1-3. Addison-Wesley.

Kowalski, R. 1979. Algorithm = logic + control. Commununications of the ACM 22, 7, 424-436.

KrÄMER, E. 2001. A generic search engine for a Java Constraint Kit. Diplomarbeit. Inst. of Comp. Sc., LMU, Munich, Germany.

Krishnamurthy, R., Boral, H., and Zaniolo, C. 1986. Optimization of nonrecursive queries. In VLDB'86: Proc. 12th Intl. Conf. Very Large Data Bases, Kyoto, Japan, pp. 128-137.

Lam, E. S. And Sulzmann, M. 2006. Towards agent programming in CHR. In CHR'06, Schrijvers and Frühwirth (2006), pp. 17-31.

Lam, E. S. And Sulzmann, M. 2007. A concurrent Constraint Handling Rules semantics and its implementation with software transactional memory. In DAMP'07: Proc. ACM SIGPLAN Workshop on Declarative Aspects of Multicore Programming, Nice, France, N. Glew and G. E. Blelloch, Eds.

LÖtzbeyer, H. And Pretschner, A. 2000. AutoFocus on constraint logic programming. In LPSE'00: Proc. Intl. Workshop on (Constraint) Logic Programming and Software Engineering, London, United Kingdom.

Marriott, K. And Stuckey, P. J. 1998. Programming with Constraints: an Introduction. MIT Press.

Mazur, N. 2004. Compile-time garbage collection for the declarative language Mercury. Ph.D. thesis, K.U.Leuven, Belgium.

Mazur, N., Ross, P., Janssens, G., And Bruynooghe, M. 2001. Practical aspects for a working compile time garbage collection system for Mercury. In ICLP'01, Paphos, Cyprus, pp. 105-119, P. Codognet, Ed. LNCS, vol. 2237.

MCAllester, D. A. 1999. The complexity analysis of static analyses. In $S A S^{\prime} 99$ : Proc. 6th Intl. Symp. Static Analysis, Venice, Italy, pp. 312-329.

Meister, M. 2006. Fine-grained parallel implementation of the preflow-push algorithm in CHR. In WLP'06, Fink et al. (2006), pp. 172-181.

Meister, M., Djelloul, K., And Frühwirth, T. 2006. Complexity of a CHR solver for existentially quantified conjunctions of equations over trees. In CSCLP'06: Proc. 11th Annual ERCIM Workshop on Constraint Solving and Constraint Logic Programming, Caparica, Portugal, pp. 139-153, F. Azevedo et al., Eds. LNCS, vol. 4651.

Meister, M., Djelloul, K., And Robin, J. 2007. A unified semantics for Constraint Handling Rules in transaction logic. In LPNMR'0\%: Proc. 9th Intl. Conf. 
Logic Programming and Nonmonotonic Reasoning, Tempe, AZ, USA, pp. 201213, C. Baral et al., Eds. LNCS, vol. 4483.

Menezes, L., Vitorino, J., And Aurelio, M. 2005. A high performance CHR ${ }^{\vee}$ execution engine. In $C H R^{\prime} 05$, Schrijvers and Frühwirth (2005b), pp. 35-45.

Minsky, M. L. 1967. Computation: finite and infinite machines. Prentice Hall.

Miranker, D. P., Brant, D. A., Lofaso, B., And Gadbois, D. 1990. On the performance of lazy matching in production systems. In AAAI'90: Proc. 8th National Conf. Artificial Intelligence, Boston, MA, USA, pp. 685-692, T. Dietterich and W. Swartout, Eds. MIT Press.

Morrison, D. R. 1968. PATRICIA - Practical Algorithm To Retrieve Information Coded in Alphanumeric. J. ACM 15, 4, 514-534.

Okasaki, C. 1996a. Functional data structures. In Advanced Functional Programming, 2nd Intl. School, Olympia, WA, USA, pp. 131-158, J. Launchbury, E. Meijer, and T. Sheard, Eds. LNCS, vol. 1129.

Okasaki, C. 1996b. Purely functional data structures. Ph.D. thesis, School of Computer Science, Carnegie Mellon University, Pittsburgh, PA, USA.

Okasaki, C. And Gill, A. 1998. Fast mergeable integer maps. In Workshop on $M L$, Baltimore, MD, USA, pp. 77-86.

O'Keefe, R. 1990. The Craft of Prolog. MIT Press.

Pilozzi, P. And De Schreye, D. 2008. Termination analysis of CHR revisited. In $C H R$ '08, Schrijvers et al. (2008), pp. 35-50.

Pilozzi, P., Schrijvers, T., And De Schreye, D. 2007. Proving termination of CHR in Prolog: A transformational approach. In WST'0\%: 9th Intl. Workshop on Termination, Paris, France.

Pretschner, A., Slotosch, O., Aiglstorfer, E., And Kriebel, S. 2004. Model-based testing for real. J. Software Tools for Technology Transfer 5, 2-3, 140-157.

RAISER, F. 2007. Graph transformation systems in CHR. In ICLP'07, Porto, Portugal, pp. 240-254, V. Dahl and I. Niemelä, Eds. LNCS, vol. 4670.

Raiser, F. And Frühwirth, T. 2008. Towards term rewriting systems in Constraint Handling Rules machines. In CHR'08, Schrijvers et al. (2008), pp. 19-33.

RAiser, F. AND TACChElla, P. 2007. On confluence of non-terminating CHR programs. In CHR'07, Djelloul et al. (2007), pp. 63-76.

Ribeiro, C., Zúquete, A., Ferreira, P., And Guedes, P. 2000. Security policy consistency. In RCoRP'00, London, UK, T. Frühwirth et al., Eds.

Sarna-Starosta, B. And Ramakrishnan, C. 2007. Compiling Constraint Handling Rules for efficient tabled evaluation. In PADL'0\%: Proc. 9th Intl. 
Symp. Practical Aspects of Declarative Languages, Nice, France, pp. 170-184, M. Hanus, Ed. LNCS, vol. 4354.

Sarna-Starosta, B. And Schrijvers, T. 2008. Transformation-based indexing techniques for Constraint Handling Rules. In CHR'08, Schrijvers et al. (2008), pp. $3-17$.

Sarna-Starosta, B., Zook, D., Pasalic, E., And Aref, M. 2008. Relating Constraint Handling Rules to Datalog. In CHR'08, Schrijvers et al. (2008), pp. 127-141.

Savage, J. E. 1998. Models of Computation. Addison-Wesley Longman.

SAVITCH, W. J. 1978. The influence of the machine model on computational complexity. In Interfaces between Computer Science and Operations Research, J. Lenstra, A. R. Kan, and P. van Emde Boas, Eds. Mathematical Centre Tracts, vol. 99. pp. 1-32.

SChiffel, S. AND Thielscher, M. 2007. Fluxplayer: A successful general game player. In AAAI'0\%: Proc. 22nd AAAI Conf. Artificial Intelligence, Vancouver, Canada, pp. 1191-1196. AAAI Press.

Schmauss, M. 1999. An implementation of CHR in Java. Diplomarbeit. Inst. of Comp. Sc., LMU, Munich, Germany.

Schoenmakers, B. 1992. Data structures and amortized complexity in a functional setting. Ph.D. thesis, Eindhoven Univ. of Technology, the Netherlands.

SchriJvers, T. 2004. Jmmsolve: A generative Java memory model implemented in Prolog and CHR. In ICLP'04, Saint-Malo, France, pp. 475-476, B. Demoen and V. Lifschitz, Eds. LNCS, vol. 3132.

SchriJvers, T. 2005. Analyses, optimizations and extensions of Constraint Handling Rules. Ph.D. thesis, K.U.Leuven, Belgium.

Schrijvers, T. And Demoen, B. 2004. The K.U.Leuven CHR system: Implementation and application. In $\mathrm{CHR}^{\prime} 04$, Selected Contributions, Ulm, Germany, pp. 8-12, T. Frühwirth and M. Meister, Eds.

Schrijvers, T., Demoen, B., Duck, G. J., Stuckey, P. J., And Frühwirth, T. 2006. Automatic implication checking for CHR constraints. In RULE'05: 6th Intl. Workshop on Rule-Based Programming, Nara, Japan, pp. 93-111. ENTCS, vol. $147(1)$.

SchriJvers, T. AND FrüHwirth, T. 2005a. Analysing the CHR implementation of union-find. In WCLP'05, Universität Ulm, Germany, pp. 135-146, A. Wolf, T. Frühwirth, and M. Meister, Eds. Ulmer Informatik-Berichte, vol. 2005-01.

Schrijvers, T. And Frühwirth, T., Eds. 2005b. CHR'05: Proc. 2nd Workshop on Constraint Handling Rules, Sitges, Spain. K.U.Leuven, Dept. Comp. Sc., Technical report CW421. 
SchriJvers, T. And Frühwirth, T., Eds. 2006. CHR'06: Proc. 3rd Workshop on Constraint Handling Rules, Venice, Italy. K.U.Leuven, Dept. Comp. Sc., Technical report CW452.

SchriJvers, T. And FrüHwirth, T. 2006. Optimal union-find in Constraint Handling Rules. TPLP 6, 1-2, 213-224.

Schrijvers, T., Frühwirth, T., And Raiser, F., Eds. 2008. CHR'08: Proc. 5th Workshop on Constraint Handling Rules, Hagenberg, Austria.

SchriJvers, T., Stuckey, P. J., And Duck, G. J. 2005. Abstract interpretation for Constraint Handling Rules. In PPDP'05, Lisbon, Portugal, pp. 218-229, P. Barahona and A. Felty, Eds.

SchriJvers, T. And Warren, D. S. 2004. Constraint Handling Rules and tabled execution. In ICLP'04, Saint-Malo, France, pp. 120-136, B. Demoen and V. Lifschitz, Eds. LNCS, vol. 3132.

Schrijvers, T., Warren, D. S., And Demoen, B. 2003. CHR for XSB. In CICLOPS'03: Proc. 3rd Intl. Colloq. Implementation of Constraint and Logic Programming Systems, Mumbai, India, pp. 7-20, R. Lopes and M. Ferreira, Eds. Univ. of Porto, Portugal, Dept. Comp. Sc., Tech. rep. DCC-2003-05.

Schrijvers, T., Wielemaker, J., And Demoen, B. 2005. Constraint Handling Rules for SWI-Prolog. In WCLP'05, Universität Ulm, Germany, A. Wolf, T. Frühwirth, and M. Meister, Eds. Ulmer Informatik-Berichte, vol. 2005-01.

Schrijvers, T., Zhou, N.-F., And Demoen, B. 2006. Translating Constraint Handling Rules into Action Rules. In CHR'06, Schrijvers and Frühwirth (2006), pp. $141-155$.

Seitz, C., Bauer, B., And Berger, M. 2002. Planning and scheduling in multi agent systems using Constraint Handling Rules. In IC-AI'02: Proc. Intl. Conf. Artificial Intelligence, Las Vegas, NV, USA. CSREA Press.

Selinger, P. G., Astrahan, M. M., Chamberlin, D. D., Lorie, R. A., And PricE, T. G. 1979. Access path selection in a relational database management system. In SIGMOD'r9: Proc. ACM SIGMOD Intl. Conf. Management of Data, Boston, MA, USA, pp. 23-34.

Sneyers, J. 2008. Turing-complete subclasses of CHR. In ICLP'08, Udine, Italy, M. García de la Banda and E. Pontelli, Eds. LNCS.

Sneyers, J. AND FrühwiRTh, T. 2008. Generalized CHR machines. In CHR'08, Schrijvers et al. (2008), pp. 143-157.

Sneyers, J., Schrijvers, T., And Demoen, B. 2005a. The computational power and complexity of Constraint Handling Rules. In CHR'05, Schrijvers and Frühwirth (2005b), pp. 3-17. 
Sneyers, J., Schrijvers, T., And Demoen, B. 2005b. Guard and continuation optimization for occurrence representations of CHR. In ICLP'05, Sitges, Spain, pp. 83-97, M. Gabbrielli and G. Gupta, Eds. LNCS, vol. 3668.

Sneyers, J., Schrijvers, T., And Demoen, B. 2005c. Guard reasoning for CHR optimization. Tech. rep. CW411, K.U.Leuven, Dept. Comp. Sc.

Sneyers, J., Schrijvers, T., And Demoen, B. 2005d. Guard simplification in CHR programs. In WCLP'05, Universität Ulm, Germany, pp. 123-134, A. Wolf, T. Frühwirth, and M. Meister, Eds. Ulmer Informatik-Berichte, vol. 2005-01.

Sneyers, J., Schrijvers, T., And Demoen, B. 2006a. Dijkstra's algorithm with Fibonacci heaps: An executable description in CHR. In WLP'06, Fink et al. (2006), pp. 182-191.

Sneyers, J., Schrijvers, T., And Demoen, B. 2006b. Memory reuse for CHR. In ICLP'06, Seattle, Washington, pp. 72-86, S. Etalle and M. Truszczynski, Eds. LNCS, vol. 4079.

Sneyers, J., SchriJvers, T., And Demoen, B. 2008a. The computational power and complexity of Constraint Handling Rules. To appear in ACM TOPLAS.

Sneyers, J., SchriJvers, T., And Demoen, B. 2008b. Guard reasoning in the refined operational semantics of CHR. Submitted to Special Issue of LNAI on Constraint Handling Rules.

Sneyers, J., Van Weert, P., And SchriJvers, T. 2007. Aggregates for Constraint Handling Rules. In CHR'07, Djelloul et al. (2007), pp. 91-105.

Sneyers, J., Van Weert, P., Schrijvers, T., And De Koninck, L. 2008. As time goes by: Constraint Handling Rules - A survey of CHR research between 1998 and 2007. Submitted to TPLP.

Somogyi, Z., Henderson, F., And Conway, T. 1996. The execution algorithm of Mercury, an efficient purely declarative logic programming language. J. Logic Programming 29, 1-3, 17-64.

Steinbrunn, M., Moerkotte, G., And Kemper, A. 1997. Heuristic and randomized optimization for the join ordering problem. J. VLDB 6, 3, 191-208.

Stuckey, P. J. And Sulzmann, M. 2005. A theory of overloading. ACM TOPLAS 27, 6, 1216-1269.

Sulzmann, M., Duck, G. J., Peyton-Jones, S., And Stuckey, P. J. 2007. Understanding functional dependencies via Constraint Handling Rules. J. Functional Prog. 17, 1, 83-129.

Sulzmann, M. And Lam, E. S. 2007a. Compiling Constraint Handling Rules with lazy and concurrent search techniques. In CHR'O'\%, Djelloul et al. (2007), pp. 139-149. 
Sulzmann, M. AND LAM, E. S. 2007b. Haskell - Join - Rules. In IFL'07: 19th Intl. Symp. Implementation and Application of Functional Languages, Freiburg, Germany, pp. 195-210, O. Chitil, Ed.

Sulzmann, M., Schrijvers, T., And Stuckey, P. J. 2006. Principal type inference for GHC-style multi-parameter type classes. In APLAS'06: Proc. 4th Asian Symp. on Programming Languages and Systems, Sydney, Australia, pp. 26-43, N. Kobayashi, Ed. LNCS, vol. 4279.

SwAmi, A. N. AND IYER, B. R. 1993. A polynomial time algorithm for optimizing join queries. In ICDE'93: Proc. 9th Intl. Conf. on Data Engineering, Vienna, Austria, pp. 345-354. IEEE.

TARJAN, R. AND VAN LEEUWEN, J. 1984. Worst-case analysis of set union algorithms. J. ACM 31, 2, 245-281.

Thielscher, M. 2002. Reasoning about actions with CHRs and finite domain constraints. In ICLP'02, Copenhagen, Denmark, pp. 70-84, P. J. Stuckey, Ed. LNCS, vol. 2401.

Thielscher, M. 2005. FLUX: A logic programming method for reasoning agents. In TPLP, Abdennadher et al. (2005), pp. 533-565.

Turing, A. M. 1936. On computable numbers, with an application to the Entscheidungsproblem. Proceedings of the London Mathematical Society 2, 42, 230-265.

VAN EMde BoAs, P. 1990. Machine models and simulations. In Handbook of Theoretical Computer Science, Volume A: Algorithms and Complexity, J. van Leeuwen, Ed. Elsevier.

Van Weert, P. 2008. Compiling Constraint Handling Rules to Java: A reconstruction. Tech. rep. CW521, K.U.Leuven, Dept. Comp. Sc.

VAn WeErt, P. 2008. A tale of histories. In CHR'08, Schrijvers et al. (2008), pp. 79-93.

Van Weert, P., Schrijvers, T., And Demoen, B. 2005. K.U.Leuven JCHR: a user-friendly, flexible and efficient CHR system for Java. In CHR'05, Schrijvers and Frühwirth (2005b), pp. 47-62.

Van Weert, P., Sneyers, J., And Demoen, B. 2008. Aggregates for CHR through program transformation. In LOPSTR'07, Kongens Lyngby, Denmark, A. King, Ed. LNCS, vol. 4915.

Van Weert, P., Sneyers, J., Schrijvers, T., And Demoen, B. 2006. Extending CHR with negation as absence. In CHR'06, Schrijvers and Frühwirth (2006), pp. $125-140$.

Van Weert, P., Wuille, P., Schrijvers, T., and Demoen, B. 2008. CHR for imperative host languages. Submitted to Special Issue of LNAI on Constraint Handling Rules. 
Voets, D., Pilozzi, P., And De Schreye, D. 2007. A new approach to termination analysis of Constraint Handling Rules. In $C H R^{\prime} 0 \%$, Djelloul et al. (2007), pp. $77-89$.

VolL, K. 2006. A methodology of error detection: Improving speech recognition in radiology. Ph.D. thesis, Simon Fraser University, Burnaby, Canada.

Vuillemin, J. 1978. A data structure for manipulating priority queues. Communications of the ACM 21, 4, 309-315.

Wallace, M., Novello, S., And Schimpf, J. 1997. ECL ${ }^{i}$ PS $^{e}$ : A platform for constraint logic programming. ICL Systems Journal 12, 1, 159-200.

Wolf, A. 1999. Adaptive Constraintverarbeitung mit Constraint-Handling-Rules - ein allgemeiner Ansatz zur Lösung dynamischer Constraint-probleme. Ph.D. thesis, Tech. Univ. Berlin, Germany.

Wolf, A. 2000a. Projection in adaptive constraint handling. In New Trends in Contraints, Paphos, Cyprus, pp. 318-338, K. Apt, A. Kakas, E. Monfroy, and F. Rossi, Eds. LNCS, vol. 1865.

Wolf, A. 2000b. Toward a rule-based solution of dynamic constraint hierarchies over finite domains. In $R C O R P^{\prime} 00$, London, UK, T. Frühwirth et al., Eds.

Wolf, A. 2001a. Adaptive constraint handling with CHR in Java. In CP'01, Paphos, Cyprus, pp. 256-270, T. Walsh, Ed. LNCS, vol. 2239.

Wolf, A. 2001b. Attributed variables for dynamic constraint solving. In Proc. 14th Intl. Conf. Applications of Prolog, Tokyo, Japan, pp. 211-219.

WoLF, A. 2005. Intelligent search strategies based on adaptive Constraint Handling Rules. In TPLP, Abdennadher et al. (2005), pp. 567-594.

Wolf, A., Gruenhagen, T., And Geske, U. 2000. On incremental adaptation of CHR derivations. In $J$. AAI, Holzbaur and Frühwirth (2000b), pp. 389-416.

Wuille, P., Schrijvers, T., And Demoen, B. 2007. CCHR: the fastest CHR implementation, in C. In CHR'0\%, Djelloul et al. (2007), pp. 123-137.

ZwICK, U. 2001. Exact and approximate distances in graphs - a survey. In ESA'01: Proc. 9th European Symp. on Algorithms, Århus, Denmark, pp. 33-48. 


\section{Index}

3SAT, 212

a posteriori guard, 148

a priori guard, 148

abduction, 53

abstract CHR machine, 211

abstract interpretation, 73

abstract semantics $\omega_{t}, 33$

$$
\omega_{t}^{s p} \text { semantics, } 214
$$

ACD term rewriting, 55

active, 65

acyclic join graph, 154

adaptive CHR, 43

aggregates, 42,89

ALGOL, 2, 87

algorithms in CHR, 75

amortized time complexity, 24, 83

applications of CHR, 50

arrays, 125,196

ask constraint, 4

association lists, 196

asymptotic complexity, 23

attributed variable, 122

automatic solver generation, 52

AVL-tree, 196

B-Prolog, 41

big $\mathrm{O}$ notation, 23

body, see rule

built-in constraints, 28

built-in store, 34

bushy tree, 145

C, 2, 41, 201 call-based semantics $\omega_{c}, 69$

extended $-\omega_{c}^{\prime}, 134$

CCHR, 41

Chameleon, 39

CHIP, 2

CHR, 5, 27

applications, 50

compilation, 57

example programs, 44

extensions, see extensions of -

program, see CHR program

related formalisms, 54

rules, see rule

syntax, 28

systems, 38

CHR machine, 165

— with stored program, 213

abstract -, 211

complexity, 180

confluent - 211

deterministic - 211

feasible - 211

generalized - 205

non-deterministic —, 211

refined -, 211

self-modifying —, 213

CHR program, 28

analysis, 36

completion, 37

complexity, 38

confluence, 36

example programs, 44

operational equivalence, 37 
program properties, 36

rules, see rule

termination, 37

$\mathrm{CHR}^{\mathrm{rp}}, 60,89,116,125$

$\mathrm{CHR}^{\vee}, 42$

CHR-only machine, 166

CHRat, 43

CHRG, 53

CHRSP machine, 213

Church, Alonzo, 19

Church-Turing thesis, 19

Ciao Prolog, 41

classical logic semantics, 32

CLP, 3, 4

co-NP, 26

co-NP-complete, 26, 215

co-NPC, 26, 215

Colmerauer, Alain, 2

compilation, 2, 57

optimizing -, 69

scheme, 58

compile-time garbage collection, 142, 196

completion, 37

complexity, 21, 38

- -wise completeness, 183, 191

- classes, 26

- of CHR machines, 180

- of RAM machines, 22

— of Turing machines, 22

amortized - , 24, 83

asymptotic - 23

big $\mathrm{O}$ notation, 23

constant factors, 200

meta-theorem, 38, 185, 190

space - 22

time - 22

computability, 19

computable execution strategy, 206

computation, 19

models, see models of -

computational complexity, 21 computational linguistics, 53

confluence, 36

generalized —, 209

confluent CHR machine, 211

constant factors, 200

constraint

active, 65

built-in, 28

declaration, 44, 125

determined partner, 187

head - 29

identifier, 33

kept head - 29

never-stored, 72

occurrence, 29, 66, 98

passive, 58, 66

removed head,- 29

representation, 62

store, 30,34

suspension, 62

constraint handling rules, see CHR

constraint logic programming, 3, 4

constraint programming, 2

constraint solvers, 3,51

continuation optimization, 98

Curry, 2, 39

cyclic join graph, 157

data integration, 52

dead constraint, 134

declarative programming, 2

dense_int, 125

dependency rank, 187

derivation, 34

description logic, 52

determined partner, 187

deterministic, 35

deterministic CHR machine, 211

DFA, see finite automata

Dijkstra, Edsger, 77

Dijkstra's algorithm, 60, 77, 202

disjunction, 42

DJCHR, 41, 43 
domain knowledge declaration, 97 dynamic join ordering, 146

\section{$\mathrm{ECL}^{i} \mathrm{PS}^{e}, 57$}

Einstein, Albert, 46

entailment checking, 107

equivalence, 37

Eratosthenes of Cyrene, 31

Sieve of Eratosthenes, 31, 99

Euclid of Alexandria, 95

Euclid's algorithm, 95

execution, see operational semantics

execution state, 34

indistinguishable states, 135

joinable, 36

variant, 35

visible part, 134

execution strategy, 206

computable - 206

extended call-based semantics $\omega_{c}^{\prime}, 134$

extensions of CHR, 42

adaptive $\mathrm{CHR}, 43$

aggregates, 42

disjunction, 42

negation as absence, 42, 193

search, 42

solver hierarchies, 43

fail continuation, 98

failure state, 35

feasible CHR machine, 211

Fibonacci, 46

Fibonacci heap, 78

Fibonacci numbers, 46, 84, 126

final state, 35

finite automata, 86

first-few answers, 159

flattening, 110, 124

FLUX, 52

frame-logic, 55

functional dependences, 187

functional dependency, 72

functional programming, 2, 39, 197
Gödel, Kurt, 21

generalized CHR machine, 205

generalized confluence, 209

generation of solvers, 52

goal, 34

grammars, 53

graph transformation systems, 56

greatest common divisor, 95

ground hash table, 123

groundness analysis, 73

guard, see rule

guard reasoning, 93

guard simplification, 95

guard solving, 128

HAL, 39

halting problem, 13, 206, 208

Hamiltonian path, 215

hash table, 122

ground - 123

non-ground -, 124

Haskell, 39, 197

head, see rule

head matching simplification, 97

high-level semantics $\omega_{t}, 33$

Hofstadter, Douglas, see self-reference

Hopcroft, John, 86

Hopcroft's algorithm, 86, 144

Horn clause, 3

host language, 28 sufficiently strong - 166

hProlog, 41, 114, 137, 186, 196, 227

identified constraint, 33

identifier, 33

imperative programming, 2, 41

in-place update, 130

indexing, 121, 187

initial state, 34

integration of data, 52

$\mathrm{JaCK}, 41$

Java, 41 
JCHR, 41, 125, 227

Jess, 197

join graph, 154

acyclic - 154

cyclic - 157

join ordering, 143, 186

cost model, 147

dynamic, 146

static, 146

join-calculus, 55

joinable state, 36

kept head constraint, 29

Kleene, Stephen, 19

Kowalski, Robert, 2, 219

late storage optimization, 71, 139

LEAPS, 197

left-deep tree, 144

Leonardo of Pisa, see Fibonacci

Leuven CHR system, 41

Leuven JCHR system, 41, 125

lexicographic order, 51

limbo, 136, 139

linear logic semantics, 33

linguistics, 53

logic programming, 2, 39, 196

Prolog, 2, 3

logical algorithms, 55

logical semantics, 32

classical, 32

linear logic, 33

Maude, 197

memory reuse, 129, 189

interference with late storage, 139

Mercury, 2, 196

minimizing finite automata, 86

Minsky, Marvin, 18

Minsky machine, 18, 175

mode declaration, 44, 125

models of computation, 14

CHR machine, 165
Minsky machine, 18, 175

RAM machine, 16

RASP machine, 19

Turing machine, 14 non-deterministic, 16

modularity, 43

Mozart (Oz), 2

multi-agent systems, 52

multi-headed rule, 29

natural language processing, 53

NCHR machine, 211

negation as absence, 42, 193

nested loop join, 145

never-stored constraints, 72

non-deterministic CHR machine, 211

non-deterministic Turing machine, 16

non-ground hash table, 124

non-linear constraints, 51

NP, 26, 213

NP-complete, 26, 215

NPC, 26, 215

observation analysis, 73

occurrence number, 29

occurrence subsumption, 98

occurrenced constraint, 65

operational equivalence, 37

operational semantics

abstract $-\omega_{t}, 33$

call-based $-\omega_{c}, 69$

derivation, 34

extended call-based - $\omega_{c}^{\prime}, 134$

priority $-\omega_{p}, 60,208$

refined $-\omega_{r}, 64,186,207$

stored-program - $\omega_{t}^{s p}, 214$

optimizing compilation, 69

abstract interpretation, 73

functional dependencies, 72

groundness analysis, 73

guard reasoning, 93

indexing, 121

join ordering, 143 
late storage, 71

memory reuse, 129

never-stored constraints, 72

observation analysis, 73

propagation history, 69

OWL, 52

$\mathrm{Oz}, 2$

P, 26, 216

partial join, 149

passive constraint, 58

passive occurrence, $66,72,98$

Patricia tree, 197

Peano-arithmetic RAM machine, 17

Petri nets, 56

pragma passive, 66

prime numbers, 31, 99, 112, 128

priorities, 60, 116

priority queue, 78

priority semantics $\omega_{p}, 60,208$

processing tree, 144

program analysis, 36

program generation, 52

Prolog, 2, 3, 39, 41

B-Prolog, 41

Ciao Prolog, 41

hProlog, 41, 114, 196, 227

pure -, 196

SICStus Prolog, 41, 227

SWI-Prolog, 41, 114

XSB, 41

YAP, 41

propagation history, 34, 69

PSPACE, 26

RAM machine, 16

Peano-arithmetic, 17

standard, 18

RASP machine, 19

rational trees, 51

refined CHR machine, 211

refined semantics $\omega_{r}, 64,186,207$

register initialization, 192 removed head constraint, 29

representation of constraints, 62

RETE, 197

right-deep tree, 145

rule

body, 29

guard, 29, 93

a posteriori guard, 148

a priori guard, 148

head, 29

multi-headed, 29

name, 29

priorities, 60, 116

propagation, 28

simpagation, 28

simplification, 28

syntax, 28

rule engine, 197

scheduling, 51

search, 42

self-modifying CHR, 213

$\omega_{t}^{s p}$ semantics, 214

complexity, 215

self-reference, 251

semantic web, 52

semantics

informal, 30

logical, 32

classical, 32

linear logic, 33

operational, see operational setarg/3, 62, 137, 195, 200

shortest paths, 77

SICStus Prolog, 41, 227

SiLCC, 39

soft constraints, 51

solver generation, 52

solver hierarchies, 43

space complexity, 22

spatio-temporal reasoning, 51

standard RAM machine, 18

state, 34 
static join ordering, 146

STMCHR, 39

store, 34

stored program

$\omega_{t}^{s p}$ semantics, 214

CHRSP machine, 213

RASP machine, 19

strategy class, 205

structure reuse, 142, 196

success continuation, 98

successful final state, 35

Sudoku, 49

sufficiently strong host language, 166

suspension, 136

- term, 62

allocation, 71

cache, 132, 189

fields, 62, 136

reuse, 132

SWI-Prolog, 41, 114

symmetry analysis, 98

syntax of CHR, 28

systems, 38

TaiChi, 39

tell constraint, 4

term rewriting, 55, 197

$$
\mathrm{ACD}-, 55
$$

termination, 37

testing, 54

theoretical semantics $\omega_{t}, 33$

time complexity, 22

amortized -, 24, 83

transition, 34

Turing, Alan, 14, 19

Turing machine, 14

Turing-complete, 20, 167, 170

type declaration, 44, 125

type systems, 53

union-find, 76, 202

solvers derived from -, 51 variant (execution state), 35

verification, 54

visible part, 134

von Neumann, John, 19, 21

XSB, 41

YAP, 41

zebra puzzle, 46 


\section{List of Symbols}

\begin{tabular}{|c|c|c|}
\hline$\# X$ & p. 10 & length of sequence $X$ \\
\hline$\square$ & p. 10 & empty sequence \\
\hline++ & p. 10 & sequence concatenation \\
\hline$\underline{\oplus}$ & p. 10 & multiset subset \\
\hline$\uplus$ & p. 10 & multiset union \\
\hline $\bar{\exists}$ & p. 10 & existential quantification over all free variables \\
\hline $\bar{\exists}_{V}$ & p. 10 & existential quantification over all variables not in $V$ \\
\hline$\exists_{V}$ & p. 10 & existential quantification over all variables in $V$ \\
\hline $\operatorname{vars}(X)$ & p. 10 & set of variables occurring in $X$ \\
\hline $\operatorname{CHR}(\mathcal{H})$ & p. 28 & a CHR system in host language $\mathcal{H}$ \\
\hline $\mathrm{CHR}^{\mathrm{rp}}$ & p. 60 & CHR with rule priorities \\
\hline $\mathrm{CHR}^{\vee}$ & p. 42 & CHR with disjunction \\
\hline CHRSP & p. 213 & CHR with stored program (self-modifying CHR) \\
\hline NCHR & p. 211 & non-deterministic CHR \\
\hline $\mathcal{D}_{\mathcal{H}}$ & p. 28 & built-in constraint theory \\
\hline $\mathcal{G}_{\mathcal{P}}^{\mathcal{H}}$ & p. 28 & set of goals for a CHR program $\mathcal{P}$ and host language $\mathcal{H}$ \\
\hline $\mathcal{H}$ & p. 28 & host language \\
\hline
\end{tabular}




\begin{tabular}{|c|c|c|}
\hline $\mathcal{M}$ & p. 166 & a CHR machine \\
\hline $\mathcal{P}$ & p. 28 & a CHR program \\
\hline $\mathcal{L G}$ & p. 166 & set of valid goals \\
\hline$\prec$ & p. 186 & a join ordering strategy \\
\hline$\prec_{c}^{\mathcal{P}}$ & p. 186 & partner ordering for occurrence $c$ in program $\mathcal{P}$ \\
\hline$c:: K \rightsquigarrow R$ & p. 72 & functional dependency for constraint $c$ from $K$ to $R$ \\
\hline$\omega_{c}$ & p. 69 & call-based refined operational semantics of CHR \\
\hline$\omega_{c}^{\prime}$ & p. 134 & extended call-based semantics of CHR \\
\hline$\omega_{p}$ & p. 60 & priority semantics of $\mathrm{CHR}$ \\
\hline$\omega_{r}$ & p. 64 & refined operational semantics of CHR \\
\hline$\omega_{t}$ & p. 33 & abstract (or theoretical) operational semantics of CHR \\
\hline$\omega_{t}^{s p}$ & p. 214 & abstract stored-program semantics of CHRSP \\
\hline $\mathbb{A}$ & p. 65 & execution stack (in an $\omega_{r}$ CHR execution state) \\
\hline $\mathbb{B}$ & p. 34 & built-in constraint store (in a CHR execution state) \\
\hline $\mathbb{G}$ & p. 34 & goal (in a CHR execution state) \\
\hline $\mathbb{S}$ & p. 34 & CHR constraint store (in a CHR execution state) \\
\hline $\mathbb{T}$ & p. 34 & propagation history (in a CHR execution state) \\
\hline$c \# i$ & p. 34 & identified CHR constraint \\
\hline$i d(x)$ & p. 34 & identifier: $i d(c \# i)=i$ \\
\hline $\operatorname{chr}(x)$ & p. 34 & removes identifier: $\operatorname{ch} r(c \# i)=c$ \\
\hline$c \# i: j$ & p. 65 & occurrenced identified constraint \\
\hline$\dagger c \# i$ & p. 134 & dead identified constraint \\
\hline$\Sigma^{\mathrm{CHR}}$ & p. 34 & set of all $\omega_{t}$ execution states \\
\hline$\Sigma^{i n i t}$ & p. 34 & set of initial states \\
\hline
\end{tabular}




\begin{tabular}{|c|c|c|}
\hline$\Sigma^{f i n a l}$ & p. 35 & set of final states \\
\hline$\sigma_{1} \cong \sigma_{2}$ & p. 135 & execution states $\sigma_{1}$ and $\sigma_{2}$ are indistinguishable \\
\hline$\sigma_{1} \approx \sigma_{2}$ & p. 35 & execution state $\sigma_{1}$ is a variant of $\sigma_{2}$ \\
\hline$\Psi(\sigma)$ & p. 134 & the visible part of execution state $\sigma$ \\
\hline initstate $(\mathbb{G})$ & p. 34 & initial execution state for query $\mathbb{G}$ \\
\hline$\longmapsto \mathcal{P}$ & p. 34 & transition function of the $\omega_{t}$ semantics (for program $\mathcal{P}$ ) \\
\hline$\longmapsto \omega_{x}, \mathcal{P}$ & p. 60 & transition function of the $\omega_{x}$ semantics (for program $\mathcal{P}$ ) \\
\hline$\Delta$ & p. 35 & a set of derivations \\
\hline$\Delta_{\omega_{t}}^{\mathcal{H}}(\mathcal{P})$ & p. 35 & all $\omega_{t}$ derivations for program $\mathcal{P}$, host language $\mathcal{H}$ \\
\hline$\left.\Delta\right|_{\mathbb{G}}$ & p. 35 & subset of $\Delta$ that start from initstate $(\mathbb{G})$ \\
\hline$\sigma_{0} \rightsquigarrow \Delta \sigma_{n}$ & p. 35 & $\sigma_{n}$ is a $\Delta$-output of $\sigma_{0}:\left[\sigma_{0}, \ldots, \sigma_{n}\right] \in \Delta$ \\
\hline$\xi$ & p. 206 & an execution strategy \\
\hline$\xi_{\mathrm{KUL}}$ & p. 207 & execution strategy of the Leuven CHR system \\
\hline$\Omega_{t}^{\mathcal{H}}(\mathcal{P})$ & p. 206 & set of all execution strategies for program $\mathcal{P}$ \\
\hline$\Omega(\mathcal{P})$ & p. 207 & a strategy class \\
\hline$O(g(n))$ & p. 24 & asymptotically bounded (above) by $g(n)$ \\
\hline$\tilde{O}(g(n))$ & p. 24 & asymptotically bounded by $g(n) \log ^{k} g(n)$ \\
\hline$\Theta(g(n))$ & p. 24 & asymptotically bounded above and below by $g(n)$ \\
\hline $\mathbf{P}$ & p. 26 & polynomial-time decidable languages \\
\hline PSPACE & p. 26 & polynomial-space decidable languages \\
\hline NP & p. 26 & non-deterministic polynomial-time decidable languages \\
\hline NPC & p. 26 & NP-complete \\
\hline co-NP & p. 26 & complement of NP \\
\hline co-NPC & p. 26 & co-NP-complete \\
\hline $\mathbf{N P}_{\Omega}$ & p. 213 & polynomial time on a non-deterministic $\Omega$-CHR machine \\
\hline $\mathbf{P}_{\Omega}$ & p. 213 & polynomial time on a deterministic $\Omega$-CHR machine \\
\hline
\end{tabular}




\section{Biography}

Jon Sneyers was born on the 21th of June 1981 in Leuven, Belgium. In 1999, he graduated from high school at the Sint-Albertuscollege in Haasrode. He studied Informatics (licentiaat informatica) at the K.U.Leuven and graduated magna cum laude in 2004. His master thesis was supervised by Professor Danny De Schreye and was titled "Modelling Probabilistic and Logical Musical Knowledge" ("Modelleren van Probabilistische en Logische Muziekkennis").

In August 2004 he started as a Ph.D. student under the supervision of Professor Bart Demoen, joining the research group "Declarative Languages and Artificial Intelligence" (DTAI) at the K.U.Leuven Department of Computer Science. Until the end of 2005 he was supported by the Research Foundation - Flanders (FWOVlaanderen) through projects G.0144.03 and G.0160.02. In 2006 he received a Ph.D. grant of the Institute for the Promotion of Innovation through Science and Technology in Flanders (IWT-Vlaanderen).

He visited the research group of Professor Peter J. Stuckey at the University of Melbourne, Australia in 2006 and the group of Professor Thom Frühwirth at University of Ulm, Germany in 2008. 


\section{List of Publications}

\section{Articles in international reviewed journals}

Jon Sneyers, Tom Schrijvers And Bart Demoen. The computational power and complexity of Constraint Handling Rules. To appear in ACM TOPLAS.

Jon Sneyers, Tom Schrijvers and Bart Demoen. Guard reasoning in the refined operational semantics of CHR. To appear in LNAI, Special Issue on Recent Advances in Constraint Handling Rules.

Jon Sneyers, Peter Van Weert, Tom Schrijvers and Leslie De Koninck. As time goes by: Constraint Handling Rules - A survey of CHR research from 1998 to 2007. Submitted to Theory and Practice of Logic Programming (TPLP).

\section{Contributions at international conferences}

Jon Sneyers, Tom Schrijvers and Bart Demoen. Guard and continuation optimization for occurrence representations of CHR. ICLP'05, Sitges, Spain, 2005.

Jon SNEYERs. Analysis and optimization of CHR programs. Research summary. ICLP'05 Doctoral Consortium, Sitges, Spain, 2005.

Jon Sneyers, Joost Vennekens And Danny De Schreye. Probabilisticlogical modeling of music. 8th Intl. Symp. on Practical Aspects of Declarative Languages (PADL'06), Charleston, SC, USA, 2006.

Jon Sneyers, Tom Schrijvers And Bart Demoen. Memory reuse for CHR. ICLP'06, Seattle, WA, USA, 2006.

Peter Van Weert, Jon Sneyers and Bart Demoen. Aggregates for CHR through program transformation. 17th Intl. Symp. on Logic-Based Program Synthesis and Transformation (LOPSTR'07), Kongens Lyngby, Denmark, 2007.

Jon Sneyers, Peter VAn Weert, Tom Schrijvers and Bart Demoen. Aggregates in Constraint Handling Rules. ICLP'07, Porto, Portugal, 2007.

Jon SNEYERs. Turing-complete subclasses of CHR. ICLP'08, Udine, Italy, 2008. 


\section{Contributions at international workshops}

Jon Sneyers, Tom Schrijvers and Bart Demoen. Guard simplification in CHR programs. 19th Workshop on (Constraint) Logic Programming (WCLP'05), Ulm, Germany, 2005.

Jon Sneyers, Tom Schrijvers and Bart Demoen. The computational power and complexity of Constraint Handling Rules. CHR'05, Sitges, Spain, 2005. Best Paper Award.

Jon Sneyers, Tom Schrijvers and Bart Demoen. Dijkstra's algorithm with Fibonacci heaps: An executable description in CHR. 20th Workshop on Logic Programming (WLP'06), Vienna, Austria, 2006.

Peter Van Weert, Jon Sneyers, Tom Schrijvers and Bart Demoen. Extending CHR with negation as absence. CHR'06, Venice, Italy, 2006.

Leslie De Koninck and Jon Sneyers. Join ordering for Constraint Handling Rules. CHR'07, Porto, Portugal, 2007.

Jon Sneyers, Peter Van Weert and Tom Schrijvers. Aggregates for Constraint Handling Rules. CHR'07, Porto, Portugal, 2007.

Jon Sneyers and Thom Frühwirth. Generalized CHR machines. CHR'08, Hagenberg, Austria, 2008.

\section{Technical reports}

(K.U.Leuven, Dept. Computer Science)

Jon Sneyers, Tom Schrijvers and Bart Demoen. Guard simplification in CHR programs. Report CW396, 2004.

Jon Sneyers, Tom Schrijvers and Bart Demoen. Guard reasoning for CHR optimization. Report CW411, 2005.

Jon Sneyers, Tom Schrijvers and Bart Demoen. Guard and continuation optimization for occurrence representations of CHR. Report CW420, 2005.

Jon Sneyers, Tom Schrijvers and Bart Demoen. Dijkstra's algorithm with Fibonacci heaps: An executable description in CHR. Report CW429, 2005.

Jon Sneyers, Tom Schrijvers and Bart Demoen. Suspension optimization and in-place updates for optimizing CHR compilation. Report CW433, 2005.

Peter Van Weert, Jon Sneyers, Tom Schrijvers and Bart Demoen. To $\mathrm{CHR}\urcorner$ or not to $\mathrm{CHR}\urcorner$ : Extending CHR with negation as absence. Report CW446, 2006.

Jon Sneyers, Peter Van Weert, Tom Schrijvers and Bart Demoen. Aggregates in CHR. Report CW481, 2007. 


\section{Nederlandstalige Samenvatting}





\section{Optimaliserende Compilatie en Computationele Complexiteit van Beperkingsafhandelingsregels}

\section{Beknopte samenvatting}

Constraint Handling Rules (CHR) is een declaratieve en zeer-hoog-niveau programmeertaal gebaseerd op conditionele en meerhoofdige herschrijfregels. CHR was oorspronkelijk specifiek ontwikkeld voor het toevoegen van gebruikergedefinieerde beperkingsoplossers aan een gastheer-taal. Gedurende het laatste decennium is CHR geëvolueerd naar een krachtige en elegante taal voor algemeen gebruik, met een breed spectrum aan toepassingsdomeinen.

Computationele complexiteitstheorie is de studie van de schaalbaarheid van computerprogramma's in termen van de computationele middelen die ze nodig hebben - in het bijzonder: uitvoeringstijd en geheugengebruik.

In deze verhandeling onderzoeken we CHR vanuit het gezichtspunt van computationele complexiteitstheorie. In het eerste deel wordt een introductie gegeven op complexiteitstheorie, CHR, en compilatie van CHR. In het tweede deel verbeteren we de CHR-systemen door verschillende compiler-optimalisatietechnieken te ontwikkelen, te implementeren en experimenteel te evalueren.

Ten slotte bewijzen we in het derde deel een "complexiteitsgewijs volledigheidsresultaat". We tonen aan dat de programmeertaal CHR en huidige geavanceerde CHR-systemen dankzij optimalisatietechnieken uit deel twee voldoende efficiënt zijn, in die zin dat elk algoritme geïmplementeerd kan worden in CHR en uitgevoerd kan worden met de optimale asymptotische tijd-en ruimtecomplexiteit.

Deze samenvattende vertaling van de verhandeling volgt de globale structuur van de Engelstalige tekst; zie de figuur op pagina 8 voor een overzicht. 


\begin{tabular}{|l|l|}
\hline English & Nederlands \\
\hline complexity-wise completeness & complexiteitsgewijze volledigheid \\
computability theory & berekenbaarheidstheorie \\
computational resources & computationele middelen \\
constraint & beperking \\
constraint handling rules & beperkingsafhandelingsregels \\
constraint (logic) programming & (logisch) programmeren met beperkingen \\
constraint solver & beperkingsoplosser \\
constraint store & beperkingsvoorraad \\
functional dependency & functionele afhankelijkheid \\
host language & gastheer-taal \\
join ordering (optimization) & samenvoegingsvolgorde(verbetering) \\
join graph & samenvoegingsgraaf \\
late storage & uitgestelde bewaring \\
memory reuse & geheugenhergebruik \\
model of computation & berekeningsmodel \\
multi-headed & meerhoofdig \\
never-stored constraint & nooit-bewaarde beperking \\
propagation history & propagatiegeschiedenis \\
refined operational semantics & verfijnde operationele semantiek \\
(rule) body & (regel)lichaam \\
rule) guard & regelconditie \\
(rule) head & (regel)hoofd \\
runtime & uitvoeringstijd \\
self-modifying CHR machine & zelfwijzigende CHR-machine \\
strategy class & strategie-klasse \\
theory of computation & berekeningsleer \\
Turing-complete & Turing-volledig \\
\hline
\end{tabular}

Tabel NL.1: Vertaling van Engelstalige terminologie 
Deze laatste bladzijden bevatten een Nederlandstalige samenvatting van de verhandeling. Tabel NL.1 geeft een overzicht van een aantal Engelstalige termen die betrekkelijk vaak gebruikt worden doorheen de tekst en waarvan de vertaling mogelijks niet voor de hand liggend is.

\section{Hoofdstuk 1: Inleiding}

De eerste computers werden geprogrammeerd aan de hand van binaire machinecodes. Programmeren was een extreem langdradig en foutgevoelig proces, waarbij de programmeur numerieke machinecodes moest onthouden en geheugenadressen manueel moest uitrekenen. In het begin van de jaren '50 werden de eerste assembleertalen ontwikkeld, gebaseerd op enigszins leesbaardere afkortingen voor elementaire instructies en symbolische instructielabels. Dergelijke talen worden beschreven als zijnde zeer laag-niveau, in die zin dat ze erg dicht staan bij de onderliggende hardware waarop de programma's worden uitgevoerd.

Om de productiviteit van het programmeren te verhogen werden hoger-niveau programmeertalen ontwikkeld. Voorbeelden van dergelijke talen zijn FORTRAN (1954), ALGOL (1958), COBOL (1959), APL (1962), BASIC (1964), Pascal (1970) en $\mathrm{C}$ (1972). Deze talen worden doorgaans vertaald naar assembleertalen; het vertaalprogramma wordt een compiler genoemd en het vertaalproces heet compilatie. Bovenstaande talen worden vandaag als laag-niveau talen beschouwd, maar destijds werden ze hoog-niveau talen genoemd.

\section{Declaratief programmeren}

De programmeertalen die we hierboven opsomden zijn imperatieve talen: een programma is in essentie een reeks verwerkingsstappen, die samen een algoritme beschrijven om een bepaald probleem op te lossen. In hoger-niveau talen zijn de stappen groter - meer details worden verborgen voor de programmeur en worden afgehandeld door de compiler — zodat de programmeur gemakkelijker het overzicht kan behouden.

In alle imperatieve talen moet een programma beschrijven hoe een probleem opgelost dient te worden. Het ideaal van declaratief programmeren daarentegen is dat een programma beschrijft wat het probleem is, en niet hoe het opgelost wordt. In de plaats van een algoritme te moeten verzinnen dat een oplossing vindt, moet de programmeur enkel maar beschrijven wat de gewenste eigenschappen zijn van een oplossing — de compiler vertaalt die specificatie vervolgens naar uitvoerbare code die een oplossing zoekt. Het is duidelijk dat voor declaratieve programmeertalen de taak van de compiler veel gecompliceerder is dan voor imperatieve talen.

Declaratieve talen worden vaak ingedeeld in drie categorieën:

Functionele programmeertalen zijn gebaseerd op het evalueren van wiskundige functies. Voorbeelden zijn LISP (1958), ML (1973) en Haskell (1990). 
Logische programmeertalen zijn gebaseerd op wiskundige logica, waarbij het bewijzen van stellingen of het genereren van modellen tot een uitvoeringsmechanisme leidt. De meeste logische programmeertalen zijn gebaseerd op Prolog (1972), dat ontwikkeld werd door Alain Colmerauer in Marseille en Robert Kowalski in Edinburgh.

Programmeren met beperkingen is gebaseerd op het opleggen van allerlei soorten van beperkingen op kandidaat-oplossingen. Voorbeelden zijn ILOG CP (1993) en Gecode (2005).

Op de kruising van bovenstaande paradigma's liggen verschillende hybride talen. Enkele voorbeelden: Mercury (1995) en Curry (1996) zijn functioneel-logische programmeertalen, CHIP (1985) en $\mathrm{ECL}^{i} \mathrm{PS}^{e}$ (1992) zijn logische talen met beperkingen, en Oz/Mozart (1991) combineert de drie paradigma's.

\section{Logisch programmeren met beperkingen}

Logische programma's bestaan uit logische formules die logische predicaten definiëren. In Prolog zijn die formules van de vorm $h \leftarrow b_{1} \wedge \ldots \wedge b_{n}$ en worden Horn clausules genoemd. Het zijn implicaties waarbij het antecedent een conjunctie van atomen is en het consequent één enkel atoom is. Voor een inleiding op logisch programmeren verwijzen we naar Clocksin and Mellish (1984); meer gevorderde boeken zijn O'Keefe (1990) en Bratko (2001).

In systemen voor het programmeren met beperkingen worden ingebouwde algoritmes gebruikt om oplossingen te genereren die voldoen aan gegeven beperkingen. Bij wijze van voorbeeld: als men een beperkingsoplosser voor domeinen bestaande uit gehele getallen de beperkingen $A$ in $1 \ldots 10$ en $A * B \#=50$ aangeeft, dan verkrijgt men de oplossingen ( $A=1, B=50),(A=2, B=25),(A=5, B=10)$ en $(A=10, B=5)$. We verwijzen de lezer naar Marriott and Stuckey (1998) en Frühwirth and Abdennadher (2003) voor een inleiding op programmeren met beperkingen.

Logisch programmeren met beperkingen (CLP) is een combinatie van logisch programmeren en programmeren met beperkingen. Een CLP systeem kan bijvoorbeeld bekomen worden door Prolog uit te breiden met een oplosser voor lineaire vergelijkingen over reële getallen. Voor een introductie op CLP verwijzen we naar Jaffar and Lassez (1987), Frühwirth et al. (1992), Jaffar and Maher (1994) en Jaffar et al. (1998).

De eerste CLP systemen waren gebaseerd op de zogenaamde 'zwarte doos'aanpak. Beperkingsoplossers werden geïmplementeerd in efficiënte laag-niveau programmeertalen zoals bijvoorbeeld C. De aldus bekomen systemen waren efficiënt maar niet erg flexibel. Het was er moeilijk om een bestaande beperkingsoplosser aan te passen of uit te breiden, bijvoorbeeld om nieuwe soorten beperkingen of andere domeinen toe te voegen.

Verschillende zogenaamde 'glazen doos'-methodes zijn voorgesteld om meer aanpasbare CLP systemen te verkrijgen. Frühwirth (1998) geeft een bondig over- 
zicht. Deze methodes lieten doorgaans enkel toe om een oplosser uit te breiden over een specifiek (meestal eindig) beperkingsdomein. Om rechtstreeks eigen beperkingsdomeinen te implementeren was enkel de aanpak van Holzbaur (1992) bruikbaar, maar dit was erg langdradig en kan beschreven worden als programmeren met een 'assembleertaal voor beperkingen'.

\section{Afhandelingsregels voor beperkingen}

In het begin van de jaren '90 ontwikkelde Thom Frühwirth (1992) een nieuwe programmeertaal (of nauwkeuriger, een taaluitbreiding), genaamd Constraint Handling Rules (CHR, afhandelingsregels voor beperkingen), met als specifiek doel om beperkingsoplossers te implementeren op een hoog-niveau en declaratieve manier. Zoals de naam al aangeeft bestaan CHR-programma's uit regels om gebruikersgedefinieerde beperkingen af te handelen. De regels vereenvoudigen uiteindelijk de gebruikersgedefinieerde beperkingen naar ingebouwde beperkingen. CHR is een taaluitbreiding: het voegt nieuwe functionaliteit toe aan een bestaande programmeertaal, genaamd de gastheer-taal, die de ingebouwde beperkingen voorziet. Oorspronkelijk werd CHR vooral toegevoegd aan Prolog, maar vandaag bestaan er ook verschillende CHR-systemen in andere talen, zoals Java en Haskell.

In de loop der jaren werd het duidelijk dat CHR en zijn varianten kan gebruikt worden voor redeneersystemen in het algemeen (niet enkel beperkingsoplossers), inclusief deductie en abductie (Holzbaur and Frühwirth 2000b). Heden ten dage wordt CHR gezien als een zeer-hoog-niveau programmeertaal voor algemene doeleinden die bijzonder geschikt is voor de snelle constructie van prototypes.

Het vergroten van het werkingsgebied van CHR - van een taal voor het specifiek doel om beperkingsoplossers te implementeren tot een taal voor algemene doelen - werd mogelijk dankzij steeds efficiëntere CHR-systemen. Op zijn beurt leverde het verbeteren van de performantie van CHR-programma's voor algemene doelen (bijvoorbeeld een CHR-implementatie van het klassieke union-find algoritme) een motivatie voor de creatie van efficiëntere CHR-systemen.

\section{Doelstellingen en bijdragen}

In deze verhandeling wensen we de bruikbaarheid van CHR als een hoog-niveau programmeertaal voor algemene doelen aan te tonen en te verbeteren. Daarom is onze belangrijkste onderzoeksdoelstelling het bestuderen en verbeteren van de performantie van CHR-systemen. Om de performantie te bestuderen (in termen van zowel uitvoeringstijd als geheugengebruik) dienen we de computationele complexiteit te analyseren van de taal en zijn implementaties. Hiervoor is uiteraard een grondig onderzoek nodig van de meest geavanceerde CHR-systemen en optimalisatietechnieken die gebruikt worden. Om de performantie te verbeteren stellen we een aantal nieuwe optimalisatietechnieken voor. We zijn telkens niet enkel 
geïnteresseerd in de asymptotische complexiteiten (m.a.w. schaalbaarheid), maar ook in de constante factoren (m.a.w. absolute performantie).

Het is duidelijk dat hoog-niveau talen vele voordelen hebben - kortere ontwikkelingstijden, meer leesbare en aanpasbare programma's, enz. Anderzijds is het doorgaans het geval dat laag-niveau talen efficiënter zijn. De centrale vraag in dit onderzoek is de volgende: "wat is de prijs, in termen van berekenbaarheid en/of complexiteit, van het gebruik van een zeer-hoog-niveau taal zoals CHR in de plaats van een laag-niveau taal zoals bijvoorbeeld C ?". Dit is geen triviale vraag: sommige hoog-niveau talen (bijvoorbeeld SQL) zijn niet Turing-compleet en hebben dus behoorlijke beperkingen in termen van berekenbaarheid; andere hoog-niveau talen (bijvoorbeeld het pure gedeelte van Prolog) zijn wel Turing-compleet maar laten niet toe om elk algoritme te implementeren met de best mogelijke asymptotische tijd- en ruimtecomplexiteit (er zijn bijvoorbeeld geen efficiënte implementaties van het union-find algoritme in pure Prolog gekend). Voor wat betreft CHR zullen we in staat zijn om bovenstaande vraag op een erg gunstige manier te beantwoorden: er is geen prijs te betalen in termen van berekenbaarheid, noch in termen van asymptotische complexiteit. In termen van constante factoren is er een (waarschijnlijk onvermijdbare) prijs, maar we zullen aantonen dat die erg redelijk is - ongeveer één grootteorde qua tijd en minder dan dat qua ruimte, in huidige geavanceerde CHR-systemen.

\section{Deel I - Achtergrond}

Het eerste deel introduceert de nodige achtergrondconcepten en beschrijft de bestaande toestand op gebied van optimaliserende compilatie van CHR waarop in het volgende deel wordt verdergebouwd.

\section{Hoofdstuk 2: Complexiteitstheorie}

De berekeningsleer is één van de meest fundamentele takken van de informatica. Zij bestudeert het al dan niet oplosbaar zijn van bepaalde problemen op een algoritmische manier, zowel in principe als in de praktijk. Het deeldomein van berekenbaarheidstheorie handelt over de kwestie van algoritmische oplosbaarheid in principe, m.a.w. abstractie makende van eventuele tijd- en ruimtebeperkingen. Een belangrijk resultaat in de berekenbaarheidstheorie is de onbeslisbaarheid van het stop-probleem (Turing 1936). In het deeldomein van (computationele) complexiteitstheorie is de centrale vraag of een probleem al dan niet algoritmisch kan opgelost worden in de praktijk. Ofschoon er een algoritme kan bestaan dat het probleem in principe oplost, zou het kunnen dat het zo een enorme hoeveelheid tijd of ruimte nodig heeft - bijvoorbeeld langer dan de leeftijd van het universum of meer bits dan het aantal elementaire deeltjes — dat het in de praktijk in feite onbruikbaar is. Complexiteitstheorie richt zich hoofdzakelijk op de schaalbaarheid 
van algoritmes, m.a.w. de verhouding tussen de probleemgrootte en de hoeveelheid computationele middelen die benodigd zijn. Het bekendste open probleem in de complexiteitstheorie is de vraag of de complexiteitsklassen $\mathbf{P}$ en $\mathbf{N P}$ samenvallen.

In de berekeningsleer worden geïdealiseerde formele modellen gebruikt om de noties van "algoritme" en "computationele middelen' op een precieze manier te definiëren. In het tweede hoofdstuk bespreken we vier bekende berekeningsmodellen: de Turing-machine, de RAM-machine, de Minsky-machine en de RASP-machine. (In hoofdstuk tien introduceren we een vijfde model, namelijk de CHR-machine.) We bespreken ook enkele basisnoties van berekenbaarheidstheorie, in het bijzonder, Turing-volledigheid en de these van Church-Turing. Tenslotte definiëren we ook formeel de noties van tijd- en ruimtecomplexiteit in de verschillende berekeningsmodellen.

\section{Hoofdstuk 3: Beperkingsafhandelingsregels (CHR)}

Het derde hoofdstuk geeft een introductie op de programmeertaal CHR.

Syntax. CHR is een taaluitbreiding die gebruikergedefinieerde beperkingen toevoegt aan een gegeven gastheer-taal, die op zijn beurt voorziet in een aantal ingebouwde beperkingen. Een CHR-programma bestaat uit een reeks CHR-regels. Er zijn drie soorten regels: $(k, l, m, n \geq 1)$

- Simplificatieregels:

$$
\begin{aligned}
h_{1}, \ldots, h_{n} & \Longleftrightarrow g_{1}, \ldots, g_{m} \mid b_{1}, \ldots, b_{k} . \\
h_{1}, \ldots, h_{n} & \Longleftrightarrow g_{1}, \ldots, g_{m} \mid b_{1}, \ldots, b_{k} .
\end{aligned}
$$

- Propagatieregels:

- Simpagatieregels:

$$
h_{1}, \ldots, h_{l} \backslash h_{l+1}, \ldots, h_{n} \Longleftrightarrow g_{1}, \ldots, g_{m} \mid b_{1}, \ldots, b_{k} .
$$

De conjunctie $h_{1}, \ldots, h_{n}$ bestaat uit CHR-beperkingen en wordt het hoofd van de regel genoemd. Een regel met $n$ beperkingen in het hoofd wordt een $n$-hoofdige regel genoemd, en als $n>1$ spreekt men van een meerhoofdige regel.

De conjunctie $b_{1}, \ldots, b_{k}$ bestaat uit een mengeling van CHR-beperkingen en ingebouwde beperkingen en wordt het lichaam van de regel genoemd. Het deel van de regel dat tussen de pijl en het lichaam staat wordt de regelconditie genoemd. Het is een conjunctie van ingebouwde beperkingen. De regelconditie van onvoorwaardelijke regels ("true |") mag worden weggelaten.

Een regel wordt optioneel voorafgegaan door een naam, gevolgd door "@”. Geen twee regels mogen dezelfde naam hebben, en als een regel geen naam heeft krijgt hij een impliciete unieke naam.

Semantiek. Een afleiding begint van een een initieel verzoek, zijnde een conjunctie van beperkingen die door de gebruiker gegeven wordt. De verzameling van beperkingen wordt de beperkingsvoorraad genoemd. De afleiding gebeurt door 
het toepassen van regels uit het programma, die de beperkingsvoorraad aanpassen. Als er geen regels meer kunnen worden toegepast eindigt de afleiding; de uiteindelijke beperkingsvoorraad wordt de oplossing genoemd.

Regels passen de beperkingsvoorraad aan op de volgende manier. Een simplificatieregel kan beschouwd worden als een herschrijfregel die het hoofd van de regel vervangt door het lichaam, op voorwaarde dat de regelconditie vervuld is. De dubbele pijl geeft aan dat het hoofd logisch equivalent is aan het lichaam, hetgeen de rechtvaardiging is voor de vervanging. De bedoeling is dat het lichaam eenvoudiger is dan het hoofd.

In propagatieregels is het lichaam een gevolg van het hoofd: gegeven het hoofd mag het lichaam toegevoegd worden (indien de regelconditie vervuld is). De pijl geeft aan dat het lichaam logisch geïmpliceerd wordt door het hoofd en dus overbodig is. Toch kan het toevoegen van overbodige beperkingen soms achteraf verdere vereenvoudigingen toelaten. Simpagatieregels zijn een mengeling van simplificatieregels en propagatieregels: de beperkingen vóór de schuine streep worden behouden, terwijl de beperkingen na de streep verwijderd worden.

De volgende onderwerpen komen uitgebreid aan bod in hoofdstuk 3:

Formele semantiek. CHR heeft zowel een formele operationele semantiek (de zogenaamde abstracte operationele semantiek $\omega_{t}$ ) als een logische semantiek. Van deze laatste bestaan er twee varianten: de klassieke logische semantiek en de semantiek in lineaire logica.

Programma-eigenschappen. Een confluent programma is een programma waarvoor kan aangetoond worden dat de volgorde waarin regels worden toegepast onbelangrijk is voor het eindresultaat. Een andere belangrijke eigenschap van CHR-programma's is eindigheid.

CHR-systemen. Er bestaan CHR-systemen voor verschillende gastheer-talen. Figuur 3.2 op pagina 40 geeft een overzicht van de verschillende systemen.

Uitbreidingen van CHR. Verschillende taal-uitbreidingen zijn in de loop der tijd voorgesteld voor CHR. Voorbeelden zijn disjuncties in regellichamen, negatie en aggregatie in regelhoofden en adaptieve $\mathrm{CHR}$.

Voorbeeldprogramma's. Een aantal voorbeelden van CHR-programma's worden besproken.

Toepassingen van CHR. Verscheidene toepassingsdomeinen van CHR worden toegelicht: o.a. 'zachte' beperkingen, uurroosters berekenen, spatio-temporeel redeneren, multi-agent systemen, gegevensintegratie, typesystemen, abductie, computationele linguistiek, testen en verificatie. 
Verwante formalismes. De relatie tussen CHR en een aantal formalismes wordt besproken: o.a. join-calculus, logische algoritmes, graaf-transformatiesystemen en Petrinetten.

\section{Hoofdstuk 4: Compilatie van CHR}

In het vierde hoofdstuk wordt het standaard-compilatieschema voor CHR uitgelegd. Een formalisatie van het resulterende uitvoeringsmechanisme wordt besproken, met name de verfijnde operationele semantiek $\omega_{r}$. Vervolgens worden bondig een reeks bestaande optimalisatietechnieken toegelicht: o.a. het onderhouden van de propagatiegeschiedenis, uitgestelde bewaring, nooit-bewaarde beperkingen en functionele afhankelijkheden.

\section{Hoofdstuk 5: Implementatie van klassieke algoritmes}

CHR was oorspronkelijk ontworpen voor de implementatie van beperkingsoplossers. Tegenwoordig wordt CHR steeds vaker gebruikt als een programmeertaal voor algemene doeleinden. Schrijvers en Frühwirth (2005a, 2006) zijn een onderzoekstrend begonnen door het klassieke union-find algoritme van Tarjan and van Leeuwen (1984) in CHR te implementeren en te analyseren. Zij toonden aan dat voor dit algoritme de optimale complexiteit kan bekomen worden in CHR.

In het vijfde hoofstuk proberen we de lezer ervan te overtuigen dat CHR kan gebruikt worden als 'uitvoerbare pseudo-code' waarmee algoritmes bestudeerd en ontworpen kunnen worden en ondertussen een echt uitvoerbaar programma wordt geconstrueerd met de gewenste tijd- en ruimtecomplexiteit. We bespreken drie klassieke algoritmes en hun implementatie in CHR: het union-find algoritme, Dijkstra's kortste-pad algoritme met Fibonacci heaps, en Hopcroft's algoritme voor het minimaliseren van eindige automaten.

\section{Deel II - Optimaliserende CHR-compilatie}

In het tweede deel worden een aantal nieuwe optimalisatietechnieken beschreven.

\section{Hoofdstuk 6: Redeneren over regelcondities}

De abstracte operationele semantiek $\omega_{t}$ van CHR is erg nondeterministisch. Zo is bijvoorbeeld de volgorde waarin regels worden toegepast helemaal niet gespecifieerd. Instantiaties van de abstracte semantiek — bijvoorbeeld de verfijnde operationale semantiek — nemen gedeeltelijk het nondeterminisme weg en in die zin geven ze meer uitvoeringscontrole aan de programmeur.

Regelcondities kunnen overbodig zijn onder een meer geïnstantieerde semantiek terwijl ze noodzakelijk zijn in de abstracte semantiek. Gevorderde CHR- 
programmeurs zijn geneigd om dergelijke overbodige regelcondities weg te laten. Dit verbetert de performantie, maar het is anderzijds ook nefast voor het plaatselijk karakter van de logische lezing van CHR-regels: om de betekenis van een regel te begrijpen, wordt het nodig om het gehele programma te beschouwen, alsmede de details van de geïnstantieerde operationele semantiek. Als oplossing stellen we compiler-optimalisaties voor die automatisch overbodige regelcondities detecteren en verwijderen.

\section{Hoofdstuk 7: Indexeringstechnieken}

In een naïve implementatie van CHR zou de beperkingsvoorraad eenvoudigweg kunnen geïmplementeerd worden als één grote lijst. De performantie van een dergelijk CHR-systeem zou uiteraard erg gebrekkig zijn. Er zijn twee belangrijke redenen om meer geavanceerde gegevensstructuren te gebruiken. Ten eerste moet er bij het uitvoeren van ingebouwde beperkingen van de gastheer-taal beslist worden welke CHR-beperkingen daardoor beïnvloed worden en herbekeken dienen te worden. Gewoonlijk zijn dat er slechts enkele, en het is dus belangrijk om te vermijden om ze allemaal te moeten overlopen. Ten tweede wordt er bij het zoeken van een toepasbare regel gezocht in de beperkingsvoorraad naar beperkingen die overeenkomen met de regelhoofden.

In het zevende hoofdstuk worden er bondig enkele indexeringstechnieken besproken die gebruikt worden in CHR-implementaties om de performantie te verbeteren op de twee bovenstaande gebieden.

\section{Hoofdstuk 8: Geheugenhergebruik}

Het achtste hoofdstuk introduceert twee optimalisatietechnieken die de geheugenvoetafdruk van CHR-programma's drastisch reduceren. De reductie wordt bereikt door het hergebruik van de interne voorstelling van CHR-beperkingen.

De eerste optimalisatie, genaamd suspension reuse, is algemeen toepasbaar. Door de interne voorstelling van verwijderde CHR-beperkingen bij te houden kan deze hergebruikt worden voor nieuw toegevoegde CHR-beperkingen. Hier is echter mogelijk een kostprijs aan verbonden op gebied van tijdgebruik. De tweede optimalisatie, genaamd in-place updates, is enkel toepasbaar op regels van een specifieke vorm, met name regels waarin een verwijderd regelhoofd ook in het regellichaam voorkomt (mogelijks met andere argumenten). Indien deze optimalisatie mogelijk is leidt zij doorgaans tot een grotere reductie van het geheugengebruik (in bepaalde gevallen zelfs een reductie in de asymptotische ruimtecomplexiteit van lineair naar constant) en vaak ook tot een reductie in het tijdgebruik. 


\section{Hoofdstuk 9: Samenvoegingsvolgordeverbetering}

Het samenvoegingsvolgordeverbeteringsprobleem is het vinden van kost-optimale uitvoeringsplannen voor meerhoofdige regels. We presenteren een formeel kostenmodel voor samenvoegingen en onderzoeken de mogelijkheid voor dynamische samenvoegingsvolgordeverbetering (tijdens de uitvoering). We stellen enkele heuristieken voor ter benadering van de parameters van dit kostenmodel, zowel in het statische als in het dynamische geval. Verder bespreken we een $O(n \log n)$ optimalisatie-algoritme voor het speciaal geval van acyclische samenvoegingsgrafen. In het algemeen is het optimaliseren van de samenvoegingsvolgorde echter een NP-volledig probleem. Tenslotte identificeren we enkele klassen van cyclische samenvoegingsgrafen die kunnen worden gereduceerd tot acyclische grafen.

\section{Deel III - Berekenbaarheid en complexiteit}

Het derde en laatste deel handelt over de berekenbaarheids- en complexiteitseigenschappen van CHR.

\section{Hoofdstuk 10: CHR-machines}

In het tiende hoofdstuk definiëren we een nieuw berekeningsmodel, namelijk de CHR-machine. We tonen aan dat CHR-machines Turing-volledig zijn, en we identificeren een aantal syntactische deelverzamelingen van de CHR-taal die nog steeds Turing-volledig zijn, en een aantal die dat niet zijn. Tenslotte definiëren we de tijden ruimtecomplexiteit van CHR-machines.

\section{Hoofdstuk 11: Complexiteitsgewijze volledigheid}

In dit hoofdstuk onderzoeken we de complexiteit van CHR-machines en hun praktische implementatie in het Leuven CHR-systeem. Aangezien RAM-machines realistischer en sneller zijn dan Turing machines focussen we op RAM-machines. We tonen aan hoe een CHR-machine kan geprogrammeerd worden om op een efficiënte manier RAM-machines te simuleren. Daarna bespreken we hoe een RAM-machine op een efficiënte manier een CHR-machine kan simuleren — dit komt overeen met optimaliserende compilatie van CHR-programma's. Dit resulteert in een algemene meta-stelling over complexiteit. We passen deze meta-stelling vervolgens toe op het CHR-programma dat RAM-machines simuleert, hetgeen ons toelaat om te besluiten dat met de bestaande optimaliserende compilers, de RAM-machine simulator efficiënt kan geïmplementeerd worden. Daaruit volgt dat alle algoritmes in CHR kunnen worden geïmplementeerd met de juiste asymptotische tijden ruimtecomplexiteit.

Naast dit 'complexiteitsgewijs volledigheidsresultaat' bespreken we in het elfde hoofdstuk ook pogingen om een gelijkaardig resultaat te bekomen in verschillen- 
de andere declaratieve talen. Tenslotte meten we aan de hand van een aantal experimenten de constante factoren die 'verstopt' zitten in de asymptotische complexiteit. We besluiten dat CHR ongeveer tien keer trager is dan de laag-niveau taal $\mathrm{C}$, en ongeveer drie keer meer geheugen gebruikt.

\section{Hoofdstuk 12: Veralgemeende CHR-machines}

De CHR-machines uit hoofdstuk 10 zijn gebaseerd op de abstracte operationele semantics $\omega_{t}$. In hoofdstuk 12 veralgemenen we de notie van CHR-machine op verschillende manieren. We introduceren de begrippen strategie-klasse en veralgemeende confluentie. Vervolgens definiëren we veralgemeende CHR-machines die een arbitraire strategie-klasse volgen. We definiëren ook non-deterministische CHR-machines (naar analogie met non-deterministische Turing-machines) en zelfwijzigende CHR-machines (naar analogie met RASP-machines).

\section{Hoofdstuk 13: Besluit}

In het laatste hoofdstuk besluiten we aan de hand van een bondig overzicht van onze bijdragen en een bespreking van mogelijke richtingen voor verder onderzoek. We bespreken volgende 'grote uitdagingen' voor de CHR-onderzoeksgemeenschap:

- Programmeeromgevingen en hulpmiddelen: o.a. automatische analysewerktuigen, hulpmiddelen voor debuggen, nieuwe systemen in programmeertalen waarvoor nog geen CHR-systeem bestaat;

- Uitvoeringscontrole: o.a. regelprioriteiten;

- Parallelisme en concurrency: o.a. semantiek, analyse, implementatie en optimalisatie;

- Schaalbaarheid: o.a. enorme persistente en/of gedistribueerde beperkingsvoorraden; (dynamische) optimalisaties, ook voor varianten en uitbreidingen van CHR; incrementele compilatie, regeltoevoeging tijdens de uitvoering, reflectie; hogere-orde- en meta-programmeren; modulariteit, oplosserhiërarchieën en gestandardiseerde programmabibliotheken.

Verder argumenteren we dat CHR het potentieel heeft om een lingua franca te worden in de kruisbestuiving tussen (onderzoek in) verschillende formalismes. Tenslotte merken we op dat de zoektocht naar programmeertalen van steeds hoger niveau niet hoeft te eindigen bij CHR en dat uitbreidingen van CHR mogelijkerwijze kunnen leiden tot een nieuwe programmeertaal die CHR nog overtreft. 